\title{
Mass Properties Testing and Evaluation for the Multi-Mission Radioisotope Thermoelectric Generator
}

Frank S. Felicione

December 2009

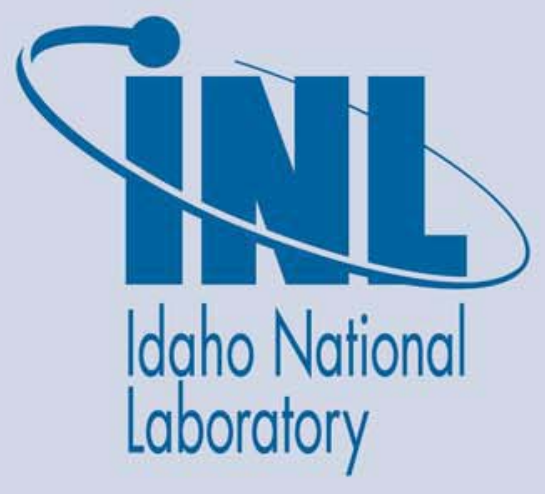

The INL is a U.S. Department of Energy National Laboratory operated by Battelle Energy Alliance 
INL/EXT-09-17458

\title{
Mass Properties Testing and Evaluation for the Multi- Mission Radioisotope Thermoelectric Generator
}

\author{
Frank S. Felicione
}

December 2009

\begin{abstract}
Idaho National Laboratory
Idaho Falls, Idaho 83415
\end{abstract}

http://www.inl.gov

\author{
Prepared for the \\ U.S. Department of Energy \\ Office of Nuclear Energy \\ Under DOE Idaho Operations Office \\ Contract DE-AC07-05ID14517
}




\section{DISCLAIMER}

This information was prepared as an account of work sponsored by an agency of the U.S. Government. Neither the U.S. Government nor any agency thereof, nor any of their employees, makes any warranty, expressed or implied, or assumes any legal liability or responsibility for the accuracy, completeness, or usefulness, of any information, apparatus, product, or process disclosed, or represents that its use would not infringe privately owned rights. References herein to any specific commercial product, process, or service by trade name, trade mark, manufacturer, or otherwise, does not necessarily constitute or imply its endorsement, recommendation, or favoring by the U.S. Government or any agency thereof. The views and opinions of authors expressed herein do not necessarily state or reflect those of the U.S. Government or any agency thereof. 
INTENTIONALLY BLANK 


\title{
Mass Properties Testing and Evaluation for the Multi-Mission Radioisotope Thermoelectric Generator
}

\author{
INL/EXT-09-17458
}

Revision 0

December 2009

Approved by:

\section{Mrank\&. Debicion}

Frank S. Felicione

Subject Matter Expert

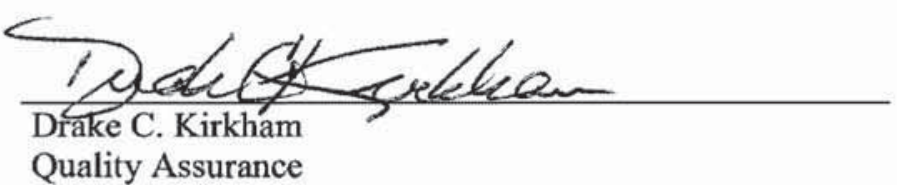

Ataphen 2\%. Aohmon for K.L. Lively
KellyL. Lively
MMRTG Project Manager

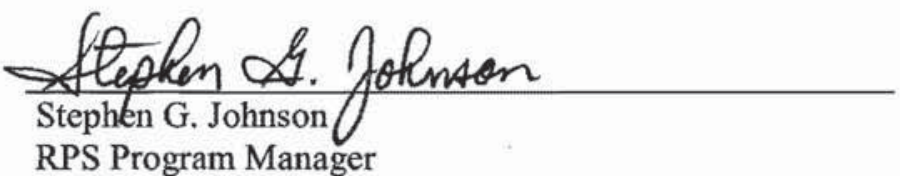

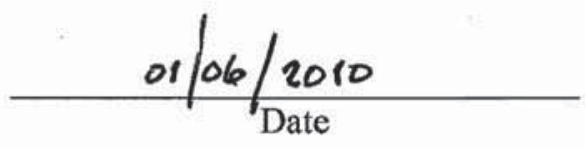

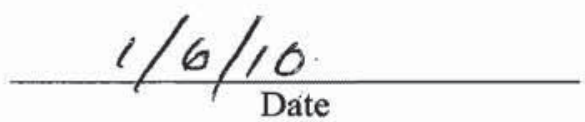

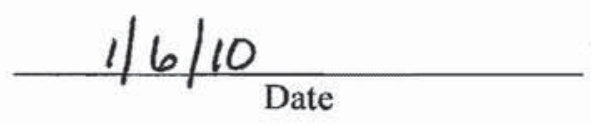


INTENTIONALLY BLANK 


\section{CONTENTS}

ACRONYMS

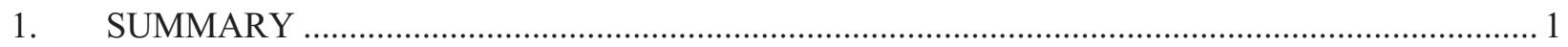

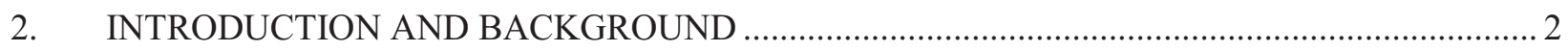

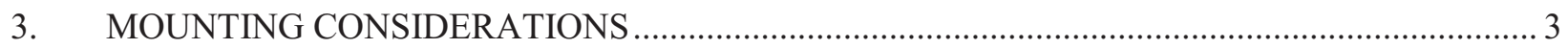

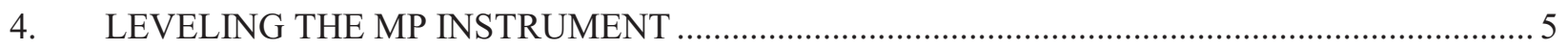

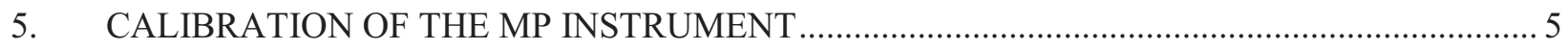

6. MOUNTING FIXTURE INSTALLATION/ALIGNMENT …….............................................. 5

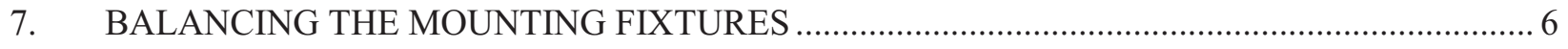

8. METROLOGICAL MEASUREMENTS TO RELATE THE MP INSTRUMENT AND

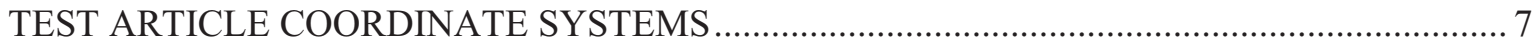

8.1 Alignment Measurements Along the X Axis ................................................................ 7

8.2 Alignment Measurements Along the Y Axis ................................................................ 8

8.3 Alignment Measurements Along the Z Axis .................................................................. 9

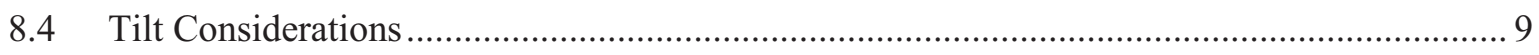

8.4.1 Linear Offsets of the MP Instrument and Test Article Coordinates ......................... 10

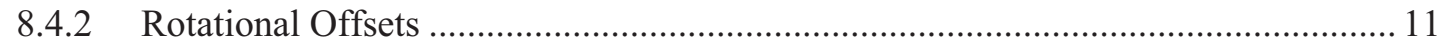

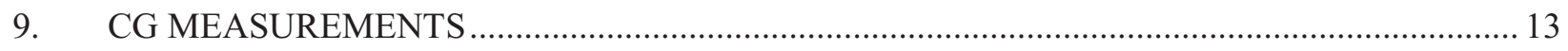

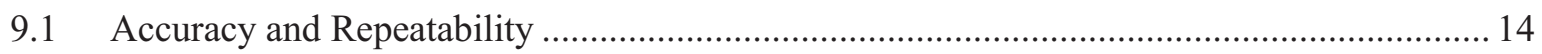

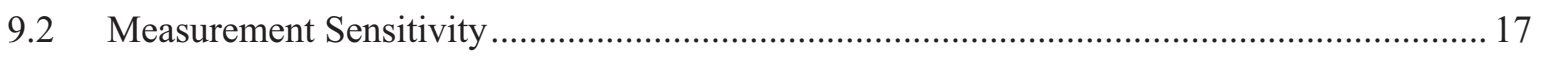

9.3 Moment Measurements Using the Space Electronics Instrument........................................ 18

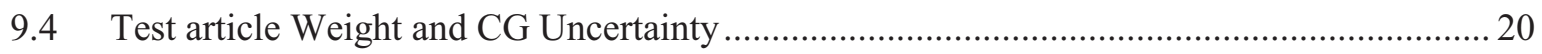

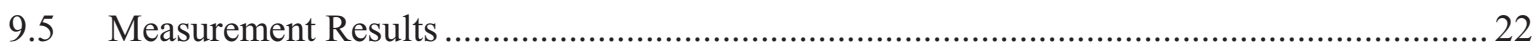

10. TEMPERATURE CHANGES IN THE MOUNTING FIXTURES …........................................ 22

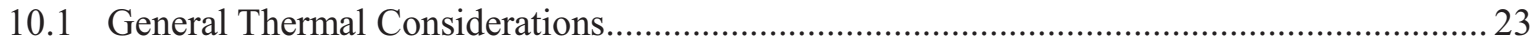

10.2 Evaluation of Fixture Temperature Variation ................................................................. 24

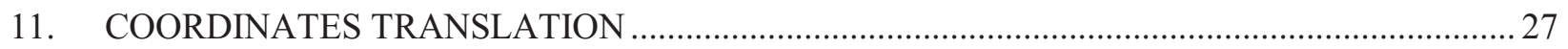

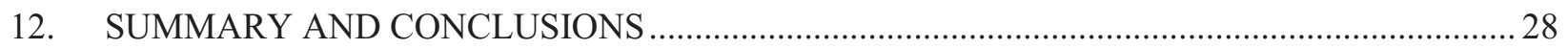

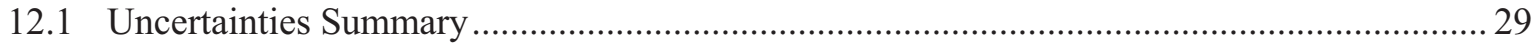

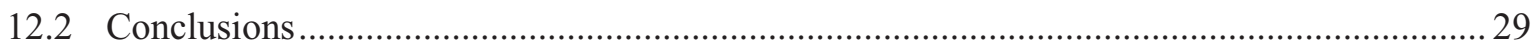

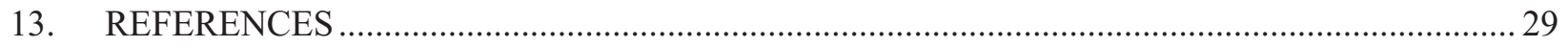


Appendix A INL Mass Properties Testing System Drawings List .................................................... 71

Appendix B Geometric Constructions for Determining Offsets of the Coordinate Systems.................... 75

Appendix C Specific Measurement System Output - Mass Standard …............................................... 85

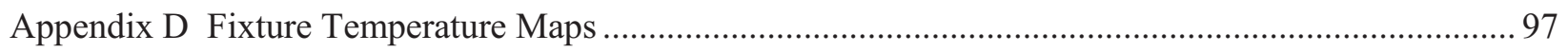

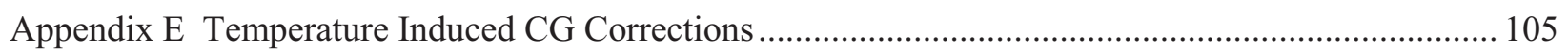

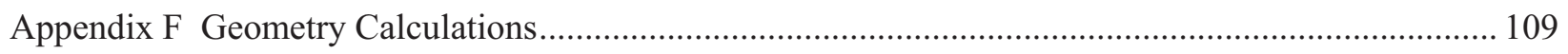

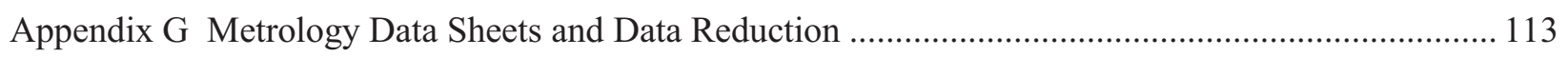

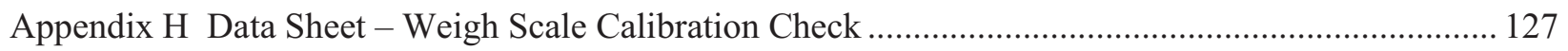

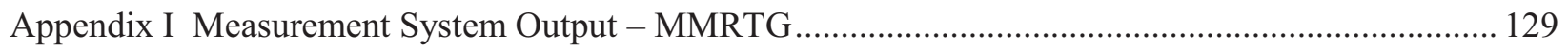

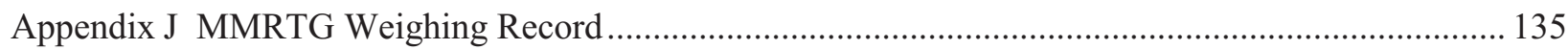

\section{FIGURES}

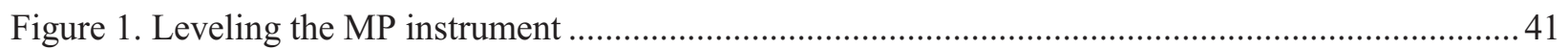

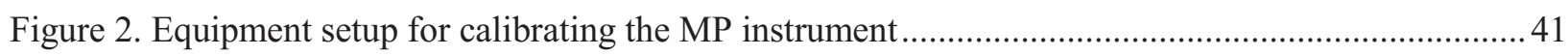

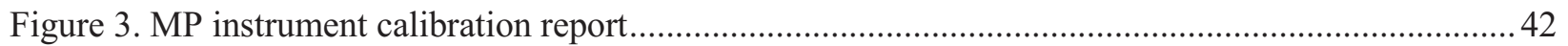

Figure 4. MP Mark-II mounting fixtures (mass standard shown installed) ............................................ 43

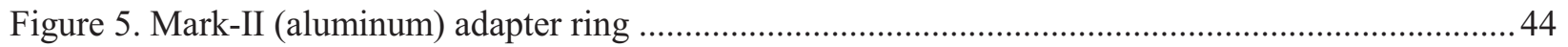

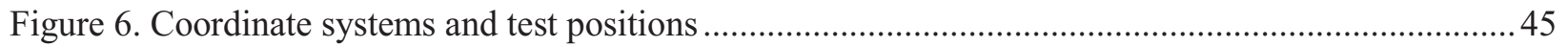

Figure 7. Final level checking of the MP mounting fixtures ................................................................. 46

Figure 8. Precision transit setup for alignment determination .............................................................. 47

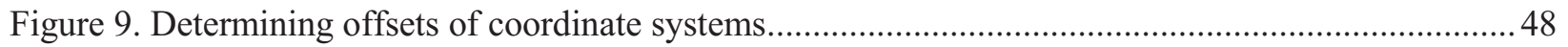

Figure 10. Typical view of lower alignment pin through precision transit .............................................. 48

Figure 11. Determining offsets of test article relative to MP instrument................................................. 49

Figure 12. Auxiliary alignment flange (pin detail superimposed) …....................................................50

Figure 13. Rotational alignment of the test article and MP instrument ................................................ 51

Figure 14. Oblique viewing angles to determine the $\mathrm{Y}$ offset of the test article coordinates ....................52

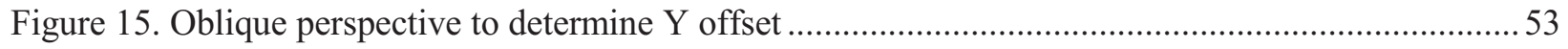

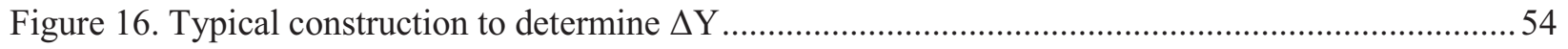

Figure 17. Tilt geometry model in X-Z view (exaggerated) ..............................................................55

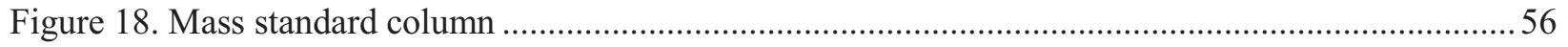

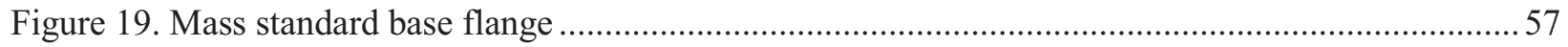

Figure 20. Pro-E software determination of CG in the mass standard..................................................58 
Figure 21. Mk-II (aluminum) adapter ring alignment features ...........................................................5

Figure 22. Measured CG location and confidence intervals for the mass standard .................................. 60

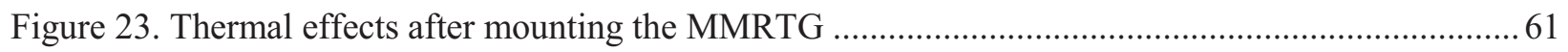

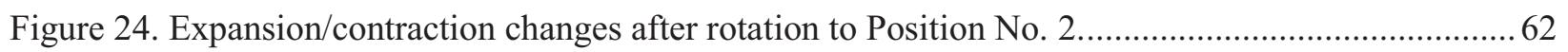

Figure 25. Temperature distributions in the rotation fixture (see temperature maps, Appendix D) ...........63

Figure 26. Key dimensions for the mounting fixtures and the test article CG position............................ 64

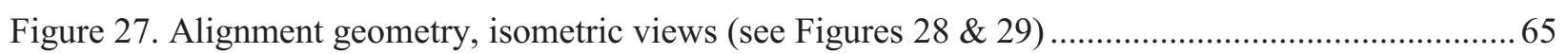

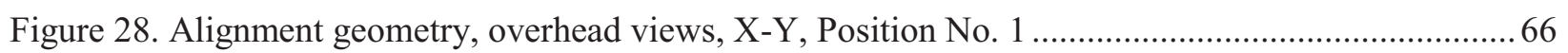

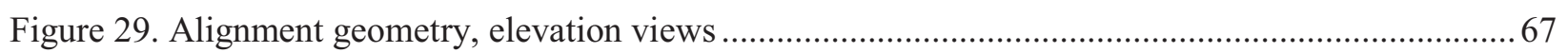

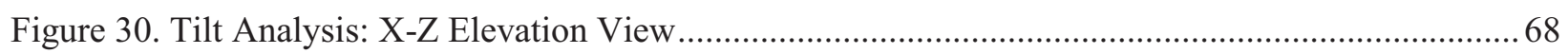

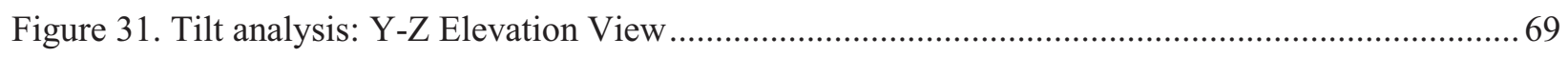

\section{TABLES}

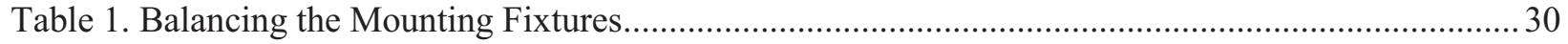

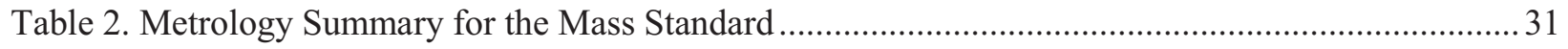

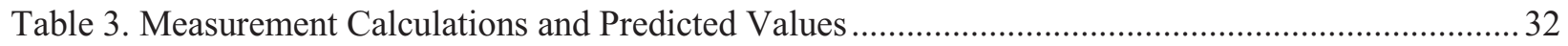

Table 4. Parametric Evaluation of CG Uncertainty Contributors .......................................................... 33

Table 5. Confidence Intervals for PART and TARE Measurements ..................................................... 34

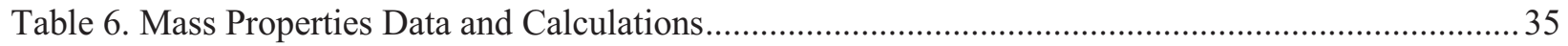

Table 7. Adjustments for Temperature Changes, (Spreadsheet E-1), Room Temp > Pre-test

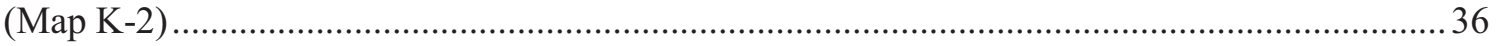

Table 8. Adjustments for Temperature Changes, (Spreadsheet E-2), Room temp > Pre-tare

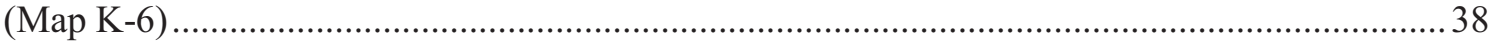

Table 9. Spreadsheet B-1 - Y Offset Constructions Characteristics ....................................................... 40 
INTENTIONALLY BLANK 


\section{ACRONYMS}

\begin{tabular}{|c|c|}
\hline A & Designator \\
\hline ASTM & American Society for Testing Materials \\
\hline B & Designator \\
\hline B209 & ASTM material configuration designator \\
\hline B211 & ASTM material configuration designator \\
\hline $\mathrm{CG}$ & Center of gravity \\
\hline $\mathrm{C}$ & Fixture alignment hole designator \\
\hline $\mathrm{D}$ & Fixture alignment hole designator \\
\hline $\mathrm{F}$ & Fahrenheit \\
\hline G & Fixture alignment hole designator \\
\hline GPHS & General Purpose Heat Source \\
\hline
\end{tabular}

$\mathrm{H} \quad$ Fixture alignment hole designator; or overall length of the mass standard

I Fixture alignment hole designator

INL Idaho National Laboratory

L Length, defined by Figure 26

M Moment

Mk Mark (version indicator)

MMRTG Multi-Mission Radioisotope Thermoelectric Generator

MOI Moment of inertia

MP Mass properties

MS Mass standard

MSL Mars Science Laboratory (Mission)

N Upper limit number

NIST National Institute of Standards and Technology

NW Northwest

P Length of locating pin; or offset of MS locating pin from cradle pin, defined in Figures 14 and 16

PWR Pratt\&Whitney Rocketdyne Co.

R Position measured from test article's origin

RTG Radioisotope Thermoelectric Generator

$\mathrm{s}^{2} \quad$ Estimate variance of a population

SSPSF Space and Security Power Systems Facility

SW Southwest

$\mathrm{S} / \mathrm{N} \quad$ Serial number 


$\begin{array}{ll}\text { T } & \text { Temperature } \\ \mathrm{t} & \text { Statistics distribution (a.k.a. Student's t) } \\ \mathrm{V} & \text { View } \\ \mathrm{W} & \text { Weight } \\ \mathrm{X} & \text { Coordinate direction } \\ \mathrm{Y} & \text { Coordinate direction } \\ \mathrm{y} & \text { Data value } \\ \mathrm{Z} & \text { Coordinate direction }\end{array}$

$\underline{\text { Subscripts }}$

\begin{tabular}{|c|c|}
\hline AR & Adapter ring \\
\hline $\mathrm{a}$ & Ancillary [items during weighing] \\
\hline bar & Indicates arithmetic average \\
\hline $\mathrm{BE}$ & Best estimate \\
\hline $\mathrm{C}$ & Cradle \\
\hline $\mathrm{CG}$ & Center of gravity (center of mass) \\
\hline ex & Excess \\
\hline $\mathrm{g}$ & Gross [weight] \\
\hline $\mathrm{i}$ & Counting index \\
\hline $\mathrm{j}$ & Counting index \\
\hline $\mathrm{L}$ & Lower locating pin \\
\hline MS & Mass Standard \\
\hline $\mathrm{O}$ & Offset \\
\hline $\mathrm{p}$ & Part \\
\hline pin & Alignment pin \\
\hline $\mathrm{R}$ & Rotation fixture \\
\hline SE & Space Electronics (Co.) \\
\hline $\mathrm{t}$ & Tare \\
\hline TA & Test article \\
\hline $\mathrm{U}$ & Upper locating pin \\
\hline
\end{tabular}


$\mathrm{X} \quad$ Coordinate direction

$\mathrm{XY} \quad$ Projection onto the X-Y plane

$\mathrm{XZ} \quad$ Projection onto the $\mathrm{X}-\mathrm{Z}$ plane

YZ Projection onto the Y-Z plane

$0 \quad$ Base measurement; or referenced to test article's coordinate system origin

2 Indicates measurement made while in Position No. 2 (see Figure 26)

\section{$\underline{\text { Superscripts }}$}

$+\quad$ Positive side

- $\quad$ Negative side

' Alternate value; or referenced to mounting fixtures' coordinates

" Referenced to test article's coordinates

\section{Greek Symbols}

$\begin{array}{ll}\alpha & \text { Coefficient of thermal expansion } \\ \beta & \text { Angle, defined by Figure } 27 \\ \Delta & \text { Difference indicator } \\ \delta & \text { Difference indicator } \\ \eta & \text { Mean value of a distribution } \\ \theta & \text { Angle, defined by Figure } 27 \\ \Phi & \text { Angle, defined by Figure } 27 \\ v & \text { Degrees of freedom (equals N-1) } \\ \xi & \text { Angle, defined by Figure } 27\end{array}$


INTENTIONALLY BLANK 


\section{Mass Properties Testing and Evaluation for the Multi-Mission Radioisotope Thermoelectric Generator}

\section{SUMMARY}

Mass properties (MP) measurements were performed for the Multi-Mission Radioisotope Thermoelectric Generator (MMRTG), serial number (S/N) 0X730401, the power system designated for the Mars Science Laboratory (MSL) mission. Measurements were made using new mounting fixtures at the mass properties testing station in the Idaho National Laboratory (INL) Space and Security Power Systems Facility (SSPSF). The objective of making mass properties measurements was to determine the generator's flight configured mass and center of mass or center of gravity (CG). Using an extremely accurate platform scale, the mass of the as-tested generator was determined to be $100.117 \pm 0.007 \mathrm{lb}$. Weight accuracy was determined by checking the platform scale with calibrated weights immediately prior to weighing the MMRTG. ${ }^{\text {a }}$

CG measurement accuracy was assessed by surrogate testing using an inert mass standard for which the CG could be readily determined analytically. Repeated testing using the mass standard enabled the basic measurement precision of the system to be quantified in terms of a physical confidence interval about the measured CG position. However, repetitious testing with the MMRTG itself was not performed in deference to the gamma and neutron radiation dose to operators and the damage potential to the flight unit from extra handling operations. Since the mass standard had been specially designed to have a total weight and CG location that closely matched the MMRTG, the uncertainties determined from its testing were assigned to the MMRTG as well. On this basis, and at the $99 \%$ confidence level, a statistical analysis found the direct, as-measured MMRTG-MSL CG to be located at $10.816 \pm 0.0011 \mathrm{in}$. measured perpendicular from the plane of the lower surface of the generator's mounting lugs ( $\mathrm{Z}$ direction), and offset from the generator's long axis centerline in the $\mathrm{X}$ and $\mathrm{Y}$ directions by $0.0968 \pm 0.0040 \mathrm{in}$. and $0.0276 \pm 0.0026$ in., respectively.

These uncertainties are based simply on the statistical treatment of results from repetitive testing performed with the mass standard and included position variations that may have occurred during several mounting/dismounting operations of both the mass standard and mounting fixtures. Because of the limited data available, the computed uncertainty intervals reported are likely, although not assuredly, wider than the intervals that would have been found had more extensive data been available. However, these uncertainties do not account for other contributors to measurement uncertainty that might be applicable. These include potential weighing errors, possible tilt of the as-mounted test article, or translation of the measurement results from the MP instrument coordinates to those of the test article. Furthermore, when testing heat producing test articles such as the MMRTG, measurement degradation can occur from thermal expansion/contraction of the mounting fixtures as they heat up or cool and cause a subtle repositioning of the test article. Analyses for such impacts were made and additional uncertainty allowances were conservatively assigned to account for these. A full, detailed description is provided in this report.

\footnotetext{
${ }^{a}$ A record of the weigh-scale calibration check is provided in Appendix $H$.
} 
After taking into account all additional potential sources of uncertainties, the best estimated, as-tested $\mathrm{CG}$, relative to the MMRTG-based coordinate system, was conservatively determined to be centered at the measured, mean position of

$$
\begin{aligned}
& X=0.0968 \mathrm{in} . \\
& Y=0.0276 \mathrm{in} . \\
& Z=10.816 \mathrm{in} .
\end{aligned}
$$

within a spherical, overall confidence interval having a conservatively estimated radius of 0.018 in., i.e., 0.036 in. diameter. Although this overall confidence interval is not amenable to a definitive, quantitative evaluation, there remains a high expectation that these results continue to be at least at the $99 \%$ level. This confidence interval is more than an order of magnitude tighter than the $10 \mathrm{~mm}(0.394 \mathrm{in}$.) diameter, allowable spherical measurement uncertainty specified for the MSL mission (Ref. 1 and Ref. 2).

Adjustments to the CG position for certain hardware differences between the testing and MSL mission flight configurations will be made by the design agency, Pratt \& Whitney Rocketdyne (PWR).

\section{INTRODUCTION AND BACKGROUND}

The MSL mission features a mobile platform (a surface-rover vehicle) that provides the support structure for various scientific experiments onboard. Electrical power for propulsion of the rover vehicle, as well as power for the experiments, is furnished by the MMRTG. The MMRTG is a ${ }^{238} \mathrm{Pu}$ powered thermoelectric generator, producing approximately $110 \mathrm{~W}$ of direct current, electrical power. Accurate knowledge of the mass properties of the spacecraft and all major equipment on board is important to ensure the flight dynamics of the spacecraft during its several months-long travel to the Mars surface are well understood so that adequate fuel for attitude control functions and maneuvering can be provided. The MMRTG weighs approximately $100 \mathrm{lb}$ and is physically packaged a considerable distance from the spacecraft centerline. This is a significant mass whose mass properties need to be factored into the overall mass properties determination for the spacecraft. Because of the importance to the mission success, a quantitative assessment of the certainty of these properties was needed. This report provides a complete account of the measurements made and the methodology used to establish the confidence bounds on the mass properties data.

Measurement uncertainty contributors arise from the measurement repeatability and sensitivity characteristics of the MP instrument, mounting position errors and unavoidable mounting variation, mass determination, and data rounding errors. A statistical treatment of repeated measurement data from the mass standard was performed to determine the associated confidence interval. This document describes the alignment metrology, the determination of the final confidence intervals, and specifies the appropriate corrections required. The test data and analyses upon which the conclusions are based are also presented.

To fully deduce the mass properties of the MMRTG, two major support activities were undertaken. These activities were

1. determine the confidence interval associated with the mass properties measurement approach and equipment to ensure the data acceptability criterion specified by the prime contractor has been satisfied; and

2. determine the physical alignment of the test article relative to the MP instrument so as to accurately translate the measurement results from the MP instrument's reference frame to that of the MMRTG.

To address both of these topics a specially constructed, dead-weight simulator, herein referred to as the mass standard, whose total mass and CG location closely mimicked those of the MMRTG, was 
constructed and tested. The simple geometry of the mass standard rendered its CG properties amenable to analysis. Favorable comparison of CG locations measured using the mass standard to those analytically determined provided a high degree of confidence in the ability of the MP instrument to accurately measure the basic mass properties of the flight generator.

An accurate knowledge of the physical position of the test article relative to the datum of the MP instrument is vital. This relationship is necessary because the MP instrument measures the CG (and moment of inertia, MOI, if it were to be performed) relative to its own datum and coordinates, whereas the spacecraft designers require these properties referenced to the mounting plane of the generator. The MMRTG does not have a handy, precision "target" from which an accurate physical relationship of the generator to the MP instrument could be readily measured. By equipping the mass standard with suitable locator features, the physical alignment could be conveniently and accurately measured.

Many close-in manipulations of the test article mounting are required to perform the multiple measurements needed to acquire a reasonable data population. Had the actual MMRTG been used to acquire the data these handling activities would have exposed personnel to significant gamma and neutron radiation doses. Instead, data gleaned from surrogate measurements on the mass standard were applied to the reduction of the actual MMRTG test data. These uncertainty estimates are, therefore, truly applicable only to static, rigid, room temperature items having a weight similar to the mass standard (approximately $100 \mathrm{lb}$ ). Application of the confidence intervals determined from the mass standard measurements to an elevated temperature test article such as the MMRTG cannot be assured off hand because of the potential for thermal expansion/contraction in the mounting fixtures. Special attention was paid to this issue to ensure the results remained valid. The measurements and analyses that provided this assurance are thoroughly described in this report.

The wider fins on the MMRTG and its bottom-only mounting configuration required fixtures that were entirely new and of a different design from those used previously. The primary motivation for developing the detailed uncertainty analyses reported herein was to establish the accuracy that could be anticipated using these new mounting fixtures in conjunction with MMRTG-sized and -configured test articles. The mounting fixtures used previously for MP testing were of an entirely different design and were about $100 \mathrm{lb}$. lighter than those for the MMRTG. The earlier test articles were also about 30\% heavier than the MMRTG. Both weight aspects contributed to a more favorable "signal-to-noise" ratio for the earlier MP measurements as compared to testing with the current equipment. Many of the measurements reported in this document were only performed to assess the capabilities of the MP instrument to make accurate measurements under these new conditions.

Having established the quantitative confidence level reported here, future MP testing of similar weight test articles that use these same fixtures need not duplicate all of these checks; a much more streamlined assessment would suffice to confirm the continued confidence level of the measurements.

\section{MOUNTING CONSIDERATIONS}

The mounting configuration of the MMRTG differs from that of the General Purpose Heat Source Radioisotope Thermoelectric Generator (GPHS-RTG) series generators previously tested at the INL's MP test station. The GPHS-RTG series generators included features that allowed them to be mounted at their mid-section during testing, although they were end mounted on the spacecraft. The MMRTG can only be end mounted via the four lugs (mounting feet) provided at the end opposite to the fueling access. The mounting fasteners comprise special socket-head machine screws inserted from underneath the fixture to which the generator is attached with washers and split, spring-loaded locking nuts installed on the generator side. Compared to the previous generators tested, this mounting configuration difference plus the wider dimensions of the heat rejection fins on the MMRTG necessitated the construction of new mounting fixtures to accommodate the MMRTG on the MP instrument. These new fixtures were designated the Mark-II ("Mk-II") MP mounting fixtures to contrast those fixtures used with the GPHS-RTG series generators. 
The MP instrument measures the CG and/or the MOI of all equipment, fixtures, fasteners, and test article(s) mounted on it relative to its own reference frame. ${ }^{b}$ The origin of the MP instrument's reference frame is centered on its axis of rotation and on the surface of the instrument's interface plate, a surface having features that allow attaching test specific hardware. A tare measurement made in the absence of the test article, but with all of the equipment other than the test article in their same positions enables the influence of these items alone to be measured and then analytically deducted by the Space Electronics Co. software. Thereafter, the results pertain only to the MP of the test article itself, but these properties are still referenced to the MP instrument's coordinate system. The design agency requires that these properties be referenced to the coordinate system established for the test article itself. For the MMRTG the coordinate system datum is at the center of an imaginary plane that contacts the bottom of its four mounting lugs. An accurate translation of the measurement data to this coordinate system was a major consideration of the MP testing.

Two alternate approaches could be employed to handle the coordinate system translation. Physical adjustment features can be included in the design of the fixtures that allow shifting the test article until the coordinate systems axes are coincident in one or more coordinate directions or the actual offsets can be measured and then analytically accounted for in the measurement translation. A combination of these two approaches was used with the mounting fixtures for the previously tested GPHS-RTG units, such as the F-8 generator, which provides electrical power for the Pluto/New-Horizons mission, launched in January, 2006 and is currently enroute to Pluto. A mechanism was provided in the GPHS-RTG fixtures that allowed shifting the test article's position along the X axis of the MP instrument until the origin of the test article, when projected onto the interface plate of the MP instrument, was coincident to that of the MP instrument. The $\mathrm{Z}$ coordinate (long axis) offset, however, was measured and then used to analytically translate the CG measurement along this axis to the reference plane of the test article. The fixed, small offset ( 2 to 3 thousandths of an inch) along the MP instrument's Y axis was, in principle, also analytically correctable, but in practice was simply accepted. Vertical tilt of the F-8 generator was carefully measured and the measured CG location was then analytically adjusted to account for any tilting.

Although adjustable positioning simplifies interpreting the test data, it requires a more complex mounting design and makes for more tedious, time consuming configuration. For the radioactive RTGs, manipulating adjustable mountings subjects personnel to considerable radiation dose. The Mk-II mounting fixtures for end-mounted RTGs were therefore designed without any field adjustable features. In order to analytically translate the measured mass properties to the test article's reference frame, the precise offset of the test article's origin relative to that of the MP instrument origin must be provided by the user. Furthermore, any angular or tilted orientation or physical shift of the test article's reference frame must be accounted for off-line and appended to the reported measurement results.

The test article's coordinate system must be orthogonal. Mounting the device such that two of its axes form a plane that is parallel to the surface of the MP instrument interface plate simplifies the measurement; otherwise orientation of the test article's axes is not restricted. The coordinate systems for both the MP instrument and for the test article may be expressed in either Cartesian or polar coordinates. Once the relationship of the coordinate systems has been specified, the MP instrument software can report the measurement results relative to both the MP instrument as well as to the test article. These results are expressed in both Cartesian and polar coordinate systems.

\footnotetext{
${ }^{\mathrm{b}}$ MOI properties for the MMRTG were to be estimated analytically by PWR therefore they were not measured by INL.
} 


\section{LEVELING THE MP INSTRUMENT}

The MP instrument measures the offsets of the test article in the horizontal plane, i.e., perpendicular to the gravity field. The instrument can neither sense nor compensate for a vertical tilt of the test article; such corrections for any tilt require a manual correction to the measurement results. Providing a level instrument surface to ensure a plumb mounting to begin with simplifies any post-test data adjustments.

The MP instrument interface plate is the horizontal surface upon which the test article and/or mounting fixtures are attached, and is the surface to be leveled. Its level was checked with a Starrett Instruments precision, calibrated level. By making repetitive, very small adjustments to the MP instrument's leveling pads, a very high degree of leveling was achieved. The smallest markings on the precision level used represent 0.0005 in. over a 12 in. span, allowing a credible resolution of 0.00025 in. over this span. Final leveling of the leveling pads on the MP instrument was made to achieve the interface plate level to within $0.00025 \mathrm{in} / \mathrm{ft}$. The photograph in Figure 1 shows the setup and typical level achieved.

\section{CALIBRATION OF THE MP INSTRUMENT}

Prior to using the MP instrument, a calibration is required. A process has been developed by the instrument manufacturer, Space Electronics Co., to accomplish this calibration in the field. A special calibration beam and two calibration weights are supplied by the instrument manufacturer for calibration. The beam has locating features for accurate positioning on the MP instrument and for placement of the calibration weights. The weights themselves carry a current National Institute of Standards and Technology (NIST)-traceable calibration. The process requires fastening the beam onto the interface plate, placing the weights on the beam according to a programmed schedule as instructed by software prompts, and executing the calibration routine. The measurement is then automatically compared to the theoretical value provided by the Space Electronics software to generate a calibration constant that is subsequently applied by the software to the measurements. An internal check compares the calibration constants to an acceptable range and indicates if the calibration is acceptable. Figure 2 depicts the calibration setup. A typical calibration report is included as Figure 3.

\section{MOUNTING FIXTURE INSTALLATION/ALIGNMENT}

The Mk-II MP mounting fixtures are comprised of three major components. The Cradle Assembly ("cradle") forms the base of the fixtures and is fastened directly to the MP instrument's interface plate. Pivoting on two upright members of the cradle is a second assembly, the Rotation Fixture. By attaching the test article to this fixture, the test article can be positioned either vertically or horizontally relative to the MP instrument. This is necessary to obtain the third component of the CG location. Position No. 1 refers to orienting the test article so that its long axis is vertical. Orientation of the test article with its long axis horizontal is referred to as Position No. 2. The test article is attached to the rotation fixture via a suitable adapter which, for the MMRTG, is the Mk-II Adapter Ring ("aluminum adapter ring"). Construction of all three mounting fixture components includes features intended to help ensure the precise, predictable, and highly repeatable positioning of the test article when mounted on the MP instrument. INL drawings 751208 and 751210, included in this report as Figures 4 and 5 respectively, depict these fixtures ${ }^{c}$. Figure 6 depicts the configuration for Positions No. 1 and No. 2 during the testing.

\footnotetext{
${ }^{\mathrm{c}}$ Appendix A lists all of the INL drawings that pertain to the MP testing system.
} 
Despite the utmost care in their fabrication and handling, minor distortions in the flatness of the cradle, rotation fixture, and adapter ring collectively degrade the overall level when stacked upon the MP instrument interface plate. Figure 7 shows the final level achieved at the top of the adapter ring mounting slides (see INL Drawing 751215 for mounting slide details). The majority of the non-level condition is traceable primarily to a very slight distortion in the flatness of the rotation fixture base.

The upper surfaces of the mounting slides provide the actual support surface for the test article. The high point is the northwest (NW) mounting slide, Slide C, while the low point is the adjacent, southwest (SW) mounting slide, Slide D, with an overall level gradient measured directly between these two mounting slides of $0.0015 \mathrm{in} / \mathrm{ft}$. Since the span between these two mounting slides is nominally only 7.85 in., the actual elevation difference $\Delta$ is proportionately less, viz.,

$$
\Delta=0.0015 \mathrm{in} . / \mathrm{ft} . * \frac{7.85 \mathrm{in} .}{12 \mathrm{in.} / \mathrm{ft} \text {. }}=0.00098 \mathrm{in} .
$$

This elevation difference creates a slight, induced tilt of the test article toward the south and west. This tilt is in addition to whatever inherent non-perpendicularity may be present in the test article itself. It is noted from Figure 7 that the net height difference computed when tracked around from the SW mounting slide to the NW mounting slide by traversing the adapter ring counter-clockwise, as viewed from above, is also $0.0015 \mathrm{in} / \mathrm{ft}$, or $0.000981 \mathrm{in}$. When compared to the measurements across the diagonals, however, there is an apparent discrepancy of $0.00025 \mathrm{in} / \mathrm{ft}$. However, when the actual spans are taken into account, the comparison of these measurements shows the actual elevation discrepancy to be only $0.0000281 \mathrm{in}$. This is well within the limiting accuracy of the precision level. For the purposes of this report, the elevation difference of 0.000981 in. is used. The discussion in Section 8.4 addresses adjustments to the measured CG position to account for this small, induced tilt characteristic.

\section{BALANCING THE MOUNTING FIXTURES}

The effects of any imbalance in the fixtures and/or test article on the measurements are canceled when the tare is made and the final test article CG calculations are performed. However, the accuracy of the final computations is increasingly degraded as imbalances in the mounted equipment become larger. Although the mounting fixtures are symmetrical by design, small issues such as minor dimensional variation among corresponding components, members, or the variation in the sizes of weld beads, etc. can affect the balance. A small brass trim weight having a mass of $0.472 \mathrm{lb}$ was fabricated to compensate for fixture imbalance. The trim weight was positioned by trial and error to minimize the residual imbalance.

Based on operational experience, a maximum imbalance acceptance criterion of 0.75 in-lb for each of the $\mathrm{X}$ and $\mathrm{Y}$ axes was stipulated. Table 1 shows the progressive improvement in the balance of the fixtures during a typical balancing session. As typified by this record, the final balance achieved was always considerably better than the acceptance criterion. 


\section{METROLOGICAL MEASUREMENTS TO RELATE THE MP INSTRUMENT AND TEST ARTICLE COORDINATE SYSTEMS}

Offset measurements of the test article coordinate system datum relative to the MP instrument datum are needed to translate the measurements from the MP instrument coordinates to those of the test article. The offsets were determined by metrological measurements using a precision optical transit equipped with a micrometer viewer, as shown in Figure 8 and sighting accurately located precision target points. The transit used was a Brunson Optical Transit, Model 76RH, equipped with a Model 160 optical micrometer viewer. The transit was set up approximately $8 \mathrm{ft}$ from the MP instrument and carefully leveled. The setup is depicted in Figure 9. A measurement resolution of approximately $0.001 \mathrm{in}$. can be routinely detected over this viewing distance. Precision, conical alignment pins were accurately placed at various positions on the equipment, as depicted in Figure 9. The sharp tips of these pins were targeted in the eyepiece of the transit. Figure 10 shows a typical view of such a pin in the transit. The pin shown in the photograph has a 1.125 in. diameter and the as-viewed spacing between the double lubber lines corresponds to a span of about 0.001 in.

Alignment was determined by measuring the lateral distances between pairs of alignment pins with the transit micrometer. One such pin was placed in the center hole (hole D) ${ }^{\mathrm{d}}$ of the cradle fixture and another pin along the $\mathrm{Y}$ coordinate $(\text { hole } \mathrm{G})^{\mathrm{e}}$. These holes are the same ones used to properly locate the cradle on the MP instrument using very close tolerance T-handle locating pins (cf., Figure 4). These peripheral holes are nominally $10 \mathrm{in}$. from the center pin and on the principal axes. During the optical alignment operations the MP instrument precision locating pins (Figure 9) actually penetrated the locator holes in both the cradle base and the MP instrument's interface plate, confirming the continued alignment of the cradle fixture to the MP instrument. The transit was focused on the tip of the pin in the center of the cradle base. The MP instrument was rotated while the center pin was observed in the transit. The sharp pointed locating pin rotated without any visible wobble, appearing to be perfectly stationary when viewed in the transit. This established that the tip of the center pin was very accurately aligned with the axis of rotation.

\subsection{Alignment Measurements Along the $\mathrm{X}$ Axis}

The transit line of sight was aligned with the $\mathrm{Y}$ axis of the cradle by viewing both the center locating pin and the locating pin on the $\mathrm{Y}$ axis. This established that the view was truly parallel to the $\mathrm{Y}$ axis and perpendicular to the $\mathrm{X}$ axis of the cradle and MP instrument. Another precision pointed locating pin was placed in the tapped hole in the center of the mass standard base protruding downward, as depicted in Figure 9. The pin featured a deliberately loose, 1 in. diameter, 8 threads/in. screw thread for attaching the pin. Accurate centering of the locating pin was achieved by providing a very snug fit to the inner cylindrical surface of a precision countersink centered on the mass standard's axis. Comparing the position of the tip of this pin to the position of the tip of the upward protruding pin in the cradle base revealed any lateral offset of the aluminum adapter ring centerline from the MP instrument's centerline in the direction perpendicular to the viewing direction as shown in Figure 11.

\footnotetext{
${ }^{\mathrm{d}}$ Locations of all alignment holes are shown in Figure 11.

${ }^{\mathrm{e}}$ Locations of all alignment holes are shown in Figure 11.
} 
The alignment described above does not ensure that the horizontal coordinates of the test article are also oriented parallel to those of the MP instrument. To check this, an auxiliary alignment flange, Figure 12 (ref. Drawing 760957), was manufactured and mounted onto the aluminum adapter ring using the same type of precision, snug-fitting titanium shoulder screws that were used to mount both the MMRTG and the mass standard. Two small, upward pointing, slip-fit alignment pins on either end of a diameter along the carefully laid out $\mathrm{Y}$ axis of this flange were provided. A central threaded countersunk hole allowed the same lower precision locating pin that was used with the mass standard to be attached, pointing downward. The transit was positioned to allow sighting along the cradle's Y axis, as described previously. The transit was then shifted laterally to sight the downward pointing alignment pin to compensate for whatever small X offset was measured in the centerlines of the mass standard and the MP instrument. The nearer of the two alignment pins protruding upward in the flange was then focused in the transit, the micrometer zeroed, and the view adjusted to measure any offset in the upward pointing pin on the opposite (far) side of the flange. This would reveal any rotational misalignment of the aluminum adapter ring and/or test article. A simple geometric construction, as shown in Figure 13, would quantify any angular misalignment relative to the MP instrument's horizontal axes. In fact, no angular misalignment of this flange was discernable.

\subsection{Alignment Measurements Along the Y Axis}

Determining the alignment between the MP instrument and test article along the $\mathrm{X}$ axis in both Positions No. 1 and No. 2 as described in the previous paragraphs is quite straightforward. In principle, determining the alignment along the $\mathrm{Y}$ axis can be performed similarly. However, when in Position No. 1 the construction of the cradle fixture causes the determination of the Y offset to be more complicated. The outrigger members of the cradle obscure the view of the axial locating pins when the transit view is directed along the $\mathrm{X}$ axis. A view somewhat oblique to the $\mathrm{X}$ axis is required. Figure 14 depicts the positions of the fixture and test article locating pins relative to the viewing direction during this measurement. There is only a narrow viewing angle, between about $16.5^{\circ}$ and $19^{\circ}$ off of the $\mathrm{X}$ axis, for which the cradle centerline locating pin and both the lower and upper locating pins in the mass standard can be viewed through the transit in a vertical plane. Using a trial and error process, the assembly was rotated until a suitable view was found. Figure 15 shows a photograph of this process underway.

Stopping at an acceptable angle is tedious and time consuming. The assembly is supported by a precision air bearing and driven by a thin torque tube so the rotation angle cannot be manually positioned. Instead, the unit must be put into a test mode and during the instrument's rotation to the next measurement quadrant and the power must be shut off at just the right moment to catch this narrow viewing window. However, when the unit is stopped, the torsion drive unloads, causing the unit to oscillate for several minutes. Since the tilt of the test article could change very slightly when the pressure to the air support bearing is interrupted, the pressure must be left on to ensure accurate alignment measurements. Unloading the torsion drive returns the rotating assembly to its neutral position so that when the mounting fixtures finally come to rest, more likely than not the viewing angle will have been found to be unsuitable, making it necessary to repeat the process. Each repeat of the rotation cycle requires $6-7$ minutes and $4-5$ cycles may be required.

A drafting protractor that had been placed on the cradle oriented with its zero mark aligned with a line along the $\mathrm{X}$ axis could be sighted through the transit to detect the measurement angle on the lubber line. When the oscillations ceased and a suitable view had finally been achieved, the offsets of the locating pins were measured with the transit micrometer, and recorded. The viewing angle was read on the protractor and entered onto the data sheet. A geometric construction from these measurements then allowed the Y offsets for both the lower and upper locating pins to be computed. Figure 16 depicts an example of this construction. 
Only a single view (Figure 14) is ostensibly required to calculate $\Delta Y$ offset. However, taking the arithmetic average from multiple measurements using several of the four possible views enhances the accuracy. Appendix B provides the detailed geometric constructions for all four viewing angles. The constructions cover all of the 16 potential configurations. Each of the four views shown in Figure 14 has four possibilities: two of these refer to the relative position of the cradle centerline pin either near the viewer or away, as determined from the $\Delta \mathrm{X}$ offset measurements and two refer to the lateral positions of the target pin relative to the cradle centerline pin as viewed either to the left of the cradle pin - Red scale on the micrometer, or to the right - Black scale. For example, if the relative positions of the pins were as depicted in Figure 14 the cradle center pin would be near the viewer in Views $V_{2}$ and $V_{3}$ but away from the viewer in Views $V_{1}$ and $V_{4}$. In Views $V_{2}$ and $V_{3}$ the target pin would be to the left of the cradle pin whereas the target is to the right in Views $V_{1}$ and $V_{4}$. Note that in View $V_{3}$, had the viewing angle $\Phi$ been somewhat greater, the target pin would have lain to the right of the cradle pin. Since each construction in Appendix $D$ is applicable to two different views, viz., Views $V_{1}$ and $V_{3}$, or Views $V_{2}$ and $V_{4}$, these eight constructions cover all 16 possible combinations. These constructions are all necessary, since the particular combination cannot be anticipated. ${ }^{\mathrm{f}}$ Table 2 summarizes the measured offsets of the centerline of the test article relative to the MP instrument as found during the metrology testing. Additional discussion of these measurements is provided in Section 8.4.1.

\subsection{Alignment Measurements Along the Z Axis}

Because of the setup and limited measurement range of the transit, it could not be used to determine the offset of the test article datum along the $\mathrm{Z}$ coordinate from the MP instrument. This offset, identified as $\mathrm{Y}_{0}$ in Figure 26, was instead deduced from the as-built dimensional inspections of the mountings as shown in the legend on the figure. As depicted in the figures (e.g., Figures 9 or 17) relative to the MP instrument, the $\mathrm{Z}$ coordinate is upward from the surface of the interface plate. The primed axes are parallel to these but their origin is centered on the test article datum, i.e., the center of the upper surfaces of the mounting slides on the aluminum adapter ring. The $\mathrm{Z}^{\prime \prime}$ coordinate shares the same datum but this coordinate is measured along the axis that lies on the test article's long axis centerline, which may be somewhat tilted from the vertical in Position No. 1 or angled from the MP instrument's Y axis in Position No. 2. These coordinates are also depicted in Figures 29-31.

The $\mathrm{Z}$ component of the $\mathrm{CG}$ is reported by the MP instrument from the measurement made while the test article is in Position No. 2. The MP instrument actually measures this coordinate along its own $+Y$ axis although it is reported as the $\mathrm{Z}$ component when this designation is entered by the user during the calculation report setup. Figure 26 depicts this measured distance as $\mathrm{Y}_{2}$ to emphasize the actual basis (Y direction, Position No. 2) for the measurement.

\subsection{Tilt Considerations}

Sections 8.1 and 8.2 discussed how the offset of the test article datum from the MP instrument datum along both horizontal axes was determined. After lining up the transit on the center locating pin in the cradle and ensuring the view perspective was as desired, i.e., perpendicular to the $\mathrm{X}$ axis or at the angle required to measure the $\mathrm{Y}$ offset, the transit was rotated upward to measure the offset of the locating pin on the upper end of the mass standard in both $\mathrm{X}$ and $\mathrm{Y}$ directions. In conjunction with the lower pin

f The method described is awkward and time-consuming. In retrospect, orienting the mass standard so as to make metrology measurements on both the lower and upper pins at the same angle is not necessary as these could just as well have been made independently. However, in lieu of the oblique-view method described, it is recommended that a small viewing window be cut through the oblique members on the cradle and through the upper counterweight stanchions to obviate this complicated approach and improve the accuracy of the $\Delta Y$ measurement at the same time. This would need to be done very carefully to avoid distorting any parts of the fixture. 
offset, these measurements revealed any tilt of the relatively tall mass standard about the horizontal axes of the MP instrument. The tilt model is depicted, very exaggerated, in Figure 17. This figure actually applies to any situation where the long axis of the test article is not perfectly parallel to the corresponding axis of the MP instrument (i.e., its $\mathrm{Z}$ axis in Position No. 1 or $\mathrm{Y}$ axis in Position No. 2). So a view similar to Figure 17 could be applied to either an elevation view showing the vertical tilt of the mass standard or an overhead (plan) view showing the angular relationship of the two coordinate systems' axes. However, the X component of the CG found from the Position No. 2 measurement is inferior to that made in Position No. 1 because the test article is physically so far removed from the MP instrument datum and therefore more prone to be affected by a tilt in the mounting. Therefore, this measurement was consistently discarded and the construction shown in Figure 17 was only used to determine the tilt of the mass standard from the vertical, i.e. Position No. 1.

The symmetry of the mass standard should show both the X" and Y" components of the CG position as measured from the centerline axis of the mass standard to be extremely small, if not actually zero. However, in addition to any unavoidable measurement variation, any CG offset that results from a tilt of the mass standard while in Position No. 1 would be sensed by the MP instrument and the X and Y components of the CG would be reported as non-zero. The as-mounted offsets along both the $\mathrm{X}$ and $\mathrm{Y}$ axes were determined by sighting the upper locating pin similarly to that described in Sections 8.1 and 8.2 for the lower pin.

Knowing the tilt allows the CG position relative to the test article's (tilted) coordinates to be calculated offline from the $\mathrm{X}$ and $\mathrm{Y}$ values reported by the MP instrument. This is further clarified by the discussion below. In discussing this, it may be anticipated from the outset that because the tilt angle during MMRTG testing is very small it is not likely to have any material effect on the test results reported by the Space Electronics software. Nevertheless, the development is of potential interest to the testing of a future test article whose coordinates cannot be aligned to those of the MP instrument.

The MP instrument measures the test article's CG offset from the MP instrument's datum, which is at the top of the interface plate and on the axis of rotation. To be useful to the design agency, this offset needs to be translated to the test article's coordinates. There are three issues that could affect the translation:

- linear offset of the test article's datum relative to the MP instrument's datum;

- rotational alignment of the test article's X and Y coordinates to those of the MP instrument, i.e., any "twist" associated with the mountings; and

- $\quad$ non-parallelism of the test article's $\mathrm{Z}$ axis relative to the $\mathrm{Z}$ axis of the MP instrument.

These issues were the subject of extensive metrological investigations for the new mounting fixtures. The approaches used and the results found are described below.

\subsubsection{Linear Offsets of the MP Instrument and Test Article Coordinates}

\section{$X$ Axis Linear Offset}

Measurement of the linear offset measurement along the $\mathrm{X}$ axis was described in Section 8.1. As indicated in that section, this measurement was straightforward. The results for the $\mathrm{X}$ offsets are summarized in Table 2.

\section{$Y$ Axis Linear Offset}

The general approach to measure the offset along the $Y$ axis was described in Section 8.2. The present discussion provides additional details as to the determination of this offset. The eight data sheets in Appendix $\mathrm{G}$ are the actual records for the metrology sessions performed to determine the offsets and other alignment features using the mass standard. The multiple measurements listed on the data sheets were performed to minimize measurement errors. The final offset values used in the calculations were the 
arithmetic averages of these data. Calculations for the $\mathrm{Y}$ offsets (the $\Delta \mathrm{Y}$ values) are also included in Appendix G.

Figure 14 shows an overhead view of the mounting fixtures with the cradle centerline pin indicated in red and one of the mass standard pins (upper or lower) shown in light green. Because of the obstruction to the view along the $\mathrm{X}$ axis as described in Section 8.2, oblique views to the $\mathrm{X}$ axis are required. The four possible views are indicated in the figure. These are numbered by the quadrant through which the view first passes, the quadrants having been numbered in the conventional way, viz., counterclockwise from the $+\mathrm{X}$ axis, as shown in the boxed designations on the figure. So View No. $1\left(\mathrm{~V}_{1}\right)$ first passes through quadrant $\mathrm{I}$, View $\mathrm{V}_{4}$ first passes through quadrant IV, etc.

The precision transit was aligned to and locked onto the cradle centerline pin and the micrometer was zeroed. For this measurement the actual viewing angle relative to the MP instrument coordinates was immaterial. The cradle centerline alignment pin was removed and the drafting protractor was carefully centered on the centerline hole, aligned with the $\mathrm{X}$ axis of the cradle. By trial and error, the MP instrument was then rotated and stopped such that both the lower and upper locating pins in the mass standard could be viewed in the transit by only adjusting the transit's vertical tilt but without rotating it left or right. Each of the upper and lower pins in the mass standard, of course, could be anywhere within any of the four quadrants. These pins are depicted in light green in Figure 14; the placement shown in this figure is markedly exaggerated, intended only for illustration. The viewing angle on the protractor could be sighted in the transit. The angle was read from the protractor and recorded on a Y measurement data sheet. The rotational position of the mounting fixture's $\mathrm{X}$ axis was indicated by circling the relevant axis nearer to the viewer on the data sheet. By rotating the transit up and down, the offsets of the mass standard's upper and lower locating pins from the cradle centerline pin were then measured with the micrometer head and recorded on the data sheet. Several readings were typically recorded and averaged to arrive at the best estimate of the positions for a given configuration.

The geometric construction for determining the pin's Y offsets from the cradle centerline pin depends on three characteristics, as were described in Section 8.2: (1) the view quadrant; (2) whether the cradle pin is "near" the viewer or "away"; and (3) whether the mass standard pin lies to the left (red side of the micrometer scale) or to the right (black side of the micrometer scale) from the cradle pin as viewed in the transit. There are 16 possible combinations of these characteristics $(4 * 2 * 2=16)$. Examination of Figure 14 shows that the only difference between views $V_{1}$ and $V_{3}$ is whether the plus $(+)$ or minus $(-)$ side of the $\mathrm{X}$ axis is closer to the viewer. This, however, does cause the signs on the $\mathrm{Y}$ offset to reverse. Accordingly, a signed unit multiplier $\mathrm{i}$ is included in the $\Delta \mathrm{Y}$ equation shown on the construction (Figure 16), where $\mathrm{i}=+1$ or -1 as indicated in the legend. Similarly, views $\mathrm{V}_{2}$ and $\mathrm{V}_{4}$ differ only in this same regard. By allowing for this axis side to be either positive or negative in a given construction, these 16 combinations can be condensed to the 8 constructions shown in Appendix B. The identification of each construction is described in the construction title. Although it may be possible to contract the 8 constructions still further, doing so could introduce considerable confusion into the quick interpretation the results.

\subsubsection{Rotational Offsets}

\section{Rotation about the Vertical Axis (Twist)}

Section 8.1 described the use of the auxiliary flange to determine if the projections of the test article's $\mathrm{X}$ and $\mathrm{Y}$ axes onto the MP instrument interface plate were parallel to the MP instrument axes. It is noted that a tilt on only one of the horizontal axes would not necessarily generate a twist; however a tilt on both horizontal axes would create a twist. Since no twist was observed, any twist would have had to be too small to be detected with the 1.56 in. tall pins used with the auxiliary flange (Figure 12). These pins could not sense the imposed twist that would have been induced by a very slight tilt in both horizontal axes.

Rotation about a Horizontal Axis (Tilt) 
Non-parallelism of the $\mathrm{Z}$ axes of the test article and the MP instrument implies a tilt exists in the vertical position of the test article. This may include a rotation about either or both of the MP instrument's horizontal axes. By measuring the offsets of both the upper and lower locating pins in the mass standard from the cradle pin, any non-trivial tilt that is present can be determined. The $37.269 \mathrm{in}$. tall mass standard (including upper and lower locating pins) significantly amplifies any tilt, enhancing the ability to detect even rather minor tilt angles.

The three orthogonal CG coordinates are reported by the MP instrument from measurements in Position No. 1 as $X_{C G}$ and $Y_{C G}$ and in Position No. 2 as $X_{C G}$ and $Z_{C G}$. The preferred value for $X_{C G}$ was consistently taken from Position No. 1 for better accuracy. Metrological sessions determined the best estimated values for $\mathrm{X}_{\mathrm{U}}, \mathrm{X}_{\mathrm{L}}, \mathrm{Y}_{\mathrm{U}}$, and $\mathrm{Y}_{\mathrm{L}}$ (see Figure 17). The CG measurements performed with the mass standard in Position No. 2 provided the value for $\mathrm{Z}_{\mathrm{CG}}$. In fact, since the $\mathrm{Z}_{\mathrm{CG}}$ position is also predictable from the mass standard's geometry, a comparison of this analytical value to that from the measurement also yields useful information on the measurement accuracy. The $\mathrm{X}_{\mathrm{U}}$ and $\mathrm{X}_{\mathrm{L}}$ offsets were measured directly; however the $\mathrm{Y}_{\mathrm{U}}$ and $\mathrm{Y}_{\mathrm{L}}$ offsets had to be determined indirectly, as was described in Section 8.2. Hence, their use in the correction of the CG position, above, also sheds information on the uncertainties associated with the methods used to deduce the offsets from the $Y$ axis using the $Y_{U}$ and $Y_{L}$ measurements.

The MP instrument reports the $\mathrm{X}, \mathrm{Y}$, and $\mathrm{Z}$ components of the $\mathrm{CG}$ position relative to its own datum and axes ("machine-referenced" CG position, as indicated in Appendix C for example, run numbers 001620 - 001624). The user must furnish the lateral offsets of the testarticle datum from the MP instrument datum in the $\mathrm{X}$ and $\mathrm{Y}$ directions in Position No. 1 and the $\mathrm{X}$ and $\mathrm{Z}$ offsets in Position No. 2, again as indicated in these same run-numbered calculation sheets. These offsets are then subtracted from the measurement in the MP instrument reference frame by the Space Electronics Co. software to yield the lateral position of the CG relative to the test article's datum. This user-referenced CG position is also reported on the Space Electronics Co. reports. However, tall objects can experience some small amount of tilt, even when carefully mounted. To ensure the most accurate CG position is found, the tilt effect should be taken into account. Since the MP instrument itself is oblivious to any tilt, a manual correction is required.

Figure 17 shows the applicable geometric configuration with the tilt greatly exaggerated and defines the symbols. As before, the unprimed coordinates are those of the MP instrument. The primed coordinates are those positioned at the origin of the mounted test article. All of the primed axes are parallel to the corresponding axes of the MP instrument with only a lateral offset of the origin. The double primed coordinates are aligned to the test article itself. The nearly perfect symmetry of the mass standard provides a high assurance that its actual CG position will lie very nearly along its long axis. The test article referenced CG position needs to be corrected from the mountings' coordinates to account for any tilt in the mountings or in the test item itself, if known). After the CG correction has been made, any residual offset of the CG from the mass standard's long axis indicates the measurement error that must be assumed to result from the mountings and/or measurement instrument and therefore, needs to be applied to the MMRTG test results as well. ${ }^{\mathrm{g}}$

g All metrology work was carried out using the mass standard. The mass standard was fabricated specifically for this purpose. Provisions were included in its design for installing accurate locating pins at the top and base for use as targets to be sighted with a precision transit. The MMRTG did not include any similar features. In the absence of any definitive information to the contrary, the assumption was made that the long axis of the MMRTG was perfectly perpendicular to its own mounting pads. This assumption justified applying whatever mounting-induced tilt that was observed with the mass standard to the MMRTG test measurements. 
The tilt is defined by the deviation from the vertical of the projection of the $\mathrm{Z}$ axis of the test article onto the $\mathrm{X}-\mathrm{Z}$ and $\mathrm{Y}-\mathrm{Z}$ elevations. Two angles define the tilt. These angles measure the extent to which the upper pin is rotated toward the positive sides of the $\mathrm{X}$ and $\mathrm{Y}$ axes from a vertical line that passes through the test article datum. In the $X-Z$ elevation view the deviation from the vertical toward the $+X$ axis is denoted by the angle $\Phi$; in the $\mathrm{Y}-\mathrm{Z}$ elevation view the deviation toward the $+\mathrm{Y}$ axis is denoted by the angle $\beta$. These angles are depicted in the constructions in Figures 27-29. Configurations in multiple views are provided in these figures, which define the dimensions and angles. More detailed constructions for the analysis are provided in Figures 30 and 31. The base of the mass standard has been omitted in these figures to reduce clutter. Lacking information to the contrary, it has been assumed that any tilt of the test article is simply a rotation about its own origin, $0^{\prime \prime}$, which remains centered on and located at the plane of its mounting surface(s). The equations that define the offsets are provided on each of the detailed figures. These equations must be solved simultaneously for the projections of $\mathrm{X}^{\prime \prime}$ and $\mathrm{Z}^{\prime \prime}$ in the $\mathrm{X}-\mathrm{Z}$ view and $\mathrm{Y}^{\prime \prime}$ and $Z^{\prime \prime}$ in the $\mathrm{Y}-\mathrm{Z}$ view. The solutions are

$$
\begin{aligned}
& X_{C G-X Z}^{\prime \prime}=\frac{\left(Z_{C G}-Z_{0}\right) \sin \phi-\left(X_{C G}-X_{0}\right) \cos \phi}{\sin ^{2} \phi-\cos ^{2} \phi} \\
& Z_{C G-X Z}^{\prime \prime}=\frac{\left(X_{C G}-X_{0}\right) \sin \phi-\left(Z_{C G}-Z_{0}\right) \cos \phi}{\sin ^{2} \phi-\cos ^{2} \phi} \\
& Y_{C G-Y Z}^{\prime \prime}=\frac{\left(Z_{C G}-Z_{0}\right) \sin \beta-\left(Y_{C G}-Y_{0}\right) \cos \beta}{\sin ^{2} \beta-\cos ^{2} \beta}
\end{aligned}
$$

and

$$
Z_{C G-Y Z}^{\prime \prime}=\frac{\left(Y_{C G}-Y_{0}\right) \sin \phi-\left(Z_{C G}-Z_{0}\right) \cos \phi}{\sin ^{2} \phi-\cos ^{2} \phi}
$$

The metrology data are documented and reduced in Appendix G. A Microsoft Excel spreadsheet was developed to methodically perform the calculations. A program listing is provided in Appendix F. The key results from the calculation are summarized in Table 2.

Once the lateral offsets for the $\mathrm{X}, \mathrm{Y}$, and $\mathrm{Z}$ axes, any tilt, and the angular orientation of the mounted test article in the horizontal plane have been found, the test article geometry relative to the MP instrument is fully specified. This allows the MP measurements to be translated to the test article coordinate system, and enables offsets at any other position on the test article to be quickly proportioned geometrically. For example, the offset measured as described above at the tip of the lower locating pin can be corrected to the offset at the elevation of the pin's base, 3.405 in. above the lower pin tip (cf. INL drawing 751227). The offset at this elevation is the offset needed for a coordinate system, such as that of the MMRTG, whose origin is at the axial centerline of the generator and in the plane of the bottom of its mounting pads.

\section{CG MEASUREMENTS}

This section first discusses the characteristics of the measurement that are important to understanding the mechanisms by which uncertainties arise and then presents the measurement results as reported by the Space Electronics software. 


\subsection{Accuracy and Repeatability}

To assess the accuracy and repeatability of the test setup, the MMRTG mass standard was used. The mass standard is fabricated from two parts, the base plate and the column (bar), as shown in INL drawings 751224 and 75125, included in this report as Figures 18 and 19. These two parts are fastened together by a coarse-threaded connection. A precision countersink in the upper side of the base plate assures a very accurate centering of the column. Since both parts are the same material (Type 304 stainless steel), presumably having equal and uniform mass densities, the CG location for the entire assembly depends only on the geometry. Its theoretical CG location can be found from the individual part volumes and the positions of their respective volume centroids.

Despite the apparent geometric simplicity of the mass standard, the coarse screw threads joining the two parts plus the female screw threads in both ends complicate a very accurate hand calculation of each part's individual CG. The theoretical CG position for the mass standard was therefore determined from the Pro-E software used to generate the engineering drawings for this item. A printout of these results is shown in Figure 20 (note that the Pro-E model has the Y coordinate oriented in the direction of the mass standard's long axis, whereas this axis is the $\mathrm{Z}$ axis for the physical part as well as for the vertical axis of the MP instrument). The Pro-E software shows the CG to be located directly on the long axis as expected by the part's symmetry and 11.230 in. from the mounting surface (the underside of the flange). This computed location provides a standard against which to compare the CG measurement results to evaluate their accuracy. A mismatch between calculated and measured CG locations indicates the error contribution that must be assigned to the overall CG measurement uncertainty from the performance of the MP instrument itself.

Of course, the theoretical CG calculation should be based on the as-built dimensions of the mass standard, which may differ somewhat from the nominal dimensions shown on the drawings. Inspection reports following fabrication of the mass standard showed all dimensions conformed to the rather tight tolerances that had been specified in the design. It would be difficult to make the many inspection measurements sufficiently accurately to definitively contradict the volume computed on the basis of the nominal dimensions. On the basis of these dimensions, the Pro-E software calculated a volume of $340.72535 \mathrm{in}^{3}$, as shown in Figure 20. The actual measured weight of the mass standard was recorded at $97.198 \mathrm{lb}$. The scale calibration check against NIST-traceable standards prior to the weighing showed a measurement variation (measured minus the calibrated) of approximately $-0.007 \mathrm{lb}$. (see the weigh scale calibration record, Appendix H) at the $100 \mathrm{lb}$. level that approximates the weight of the mass standard and MMRTG. With this weight, the implied mass density would be $0.28529 \mathrm{lb} / \mathrm{in}^{3}$. This number rounds to the $0.29 \mathrm{lb} / \mathrm{in}^{3}$ materials handbook density value that was assigned by the draftsperson for the Type 304 stainless steel as shown in Figure 20. However, use of this relatively crude (two decimal places) density value with the Pro-E computed volume caused the total weight in Figure 20 to be calculated at $98.81035 \mathrm{lb}$. Aside from the crude density value used, any further discrepancy would probably stem from a combination of the true as-built dimensions, uncertainty in the actual depth of the pilot holes drilled on both ends for the female tapped holes, actual thread profiles and their dimensions vs. those built into the Pro-E software, and/or rounding errors. Any error in the assigned material density would, in principle, not have any effect on the CG location, since both parts are ostensibly the same material (although not from the same stock: one part is from bar stock and one is from plate stock). However, the former errors could affect the CG location. Since these issues only affected the mass standard measurements, and even then only rather slightly, any further concerns were dismissed in favor of simply rolling any residual discrepancy into an uncertainty allowance in the statistical evaluation of the mass standard results, described later. 
During the tare measurements, it is important that all fixtures and components be in exactly their same positions as during the test article measurements. This required that the mounting slides on the aluminum adapter ring and all fasteners be carefully located to replicate their test positions. Since the mounting slides need to be radially unrestrained when the elevated temperature MMRTG is mounted, a design feature and testing procedure to accurately reposition these for the tare was developed. The mounting slides were repositioned using fine-threaded locator screws and then locked in place. ${ }^{\mathrm{h}}$ The technique used is fully described in Sections 5.9.9.4 and 5.12.1.2 of the mass properties test procedure, RPS/HS-OI-28220, Rev. 0. For operational consistency, the same procedure was also used for measurements conducted with the room temperature mass standard.

The titanium mounting screws were kept segregated and inserted into the same mounting slide on the aluminum adapter ring as during the PART measurement. Spacers, having negligible mass, were used to position the mounting screws and flat washers to compensate for the displacement of the fasteners by the thickness of the test article's mounting pads. Very thin-walled, aluminum tube spacers were used with the elevated temperature MMRTG whereas plastic spacers were used with the room temperature, mass standard to preserve the more delicate aluminum spacers.

The uncorrected CG calculation results using the mass standard CG PART and TARE measurements in both Position No. 1 and Position No. 2 (long axis horizontal, with the $+\mathrm{Z}$ axis in the direction of the MP instrument's $+Y$ axis) are shown in Table 3 for the four measurement campaigns using the mass standard, viz., MS-1 through MS-4. The PART and TARE run numbers selected for the analyses are indicated and the calculation run numbers are also cited. These calculation output records, identified by their run numbers, are provided in Appendix I. Table 3 also summarizes the datum offsets of the mass standard from the MP instrument datum. This table shows that the measurement results compared very favorably to the analytical predictions. The largest mismatches in the X, Y, and Z directions were 0.0049 in. (MS-3), 0.0017 in. (MS-4), and -0.0042 in. (11.2258 in. - 11.230 in., MS-1), respectively.

Table 3 also provides a statistical analysis that reveals the confidence interval associated with the mean value of the computed CG position coordinates. An estimate of the true variance, $\sigma^{2}$, was required since the data from these four tests are far too sparse to infer the data are normally distributed. The estimate of the variance is $\mathrm{s}^{2}$, where

$$
s^{2}=\frac{1}{N-1} \sum_{i=1}^{N}\left(y_{i}-\bar{y}\right)^{2}
$$

The $\mathrm{y}_{\mathrm{i}}$ are the measured $\mathrm{CG}$ values for the $\mathrm{N}$ data points respectively and $\bar{y}$ is their arithmetic average. The estimated confidence interval uses the t-statistic (a.k.a. Student's t). These comprise a family of distributions where each distribution, $t_{v}$, pertains to a specific value of $v$, the degrees of freedom, where $v=\mathrm{N}-1$. For all $v$, these distributions are symmetrical about $\mathrm{t}_{v}=0$ and have a profile similar to that of the normal, or Gaussian, distribution. They peak at $t_{v}=0$ and have an inflexion point and approach zero on both the positive and negative axes at points increasingly removed from zero. Compared to the normal distribution, however, these distributions have more "spread", i.e., the "tails" of the distribution approach zero more slowly and the peak value is lower (the integrated value is always unity). For increasing N, the distributions approach more closely the normal distribution, becoming coincident at $\mathrm{N}=\infty$.

\footnotetext{
${ }^{\mathrm{h}}$ The capability to accurately reposition these slides for the tare measurement was the primary motivation for the construction and use of the aluminum adapter ring rather than using the PWR stainless-steel adapter ring. The fact that the aluminum adapter ring is also about $15 \mathrm{lb}$. lighter is also advantageous to the accuracy of the measurement. The aluminum adapter ring and the design features described are depicted in the rendering shown in Figure 21. Complete construction details are provided in INL Drawings 751210 and $751214-751217$.
} 
The $t_{v}$ distributions are mathematically complicated but are tabulated in standard statistics texts for a given value of $v$ and user-selected probability. For the four data points in Table 3 and with 99\% confidence interval, the value that leaves $0.5 \%$ of the integrated distribution in each tail (thus, the $1 \%$ overall), is $\mathrm{t}_{\mathrm{v}, 0.005}=5.841$.

The arithmetic average of the measurements, $\bar{y}$, is the best estimate of the mean value, $\eta$, and with a $99 \%$ confidence interval, the true value of the mean lies within the interval

$$
\eta=\bar{y} \pm t_{v, 0.005} \sqrt{\frac{s^{2}}{N}}
$$

Therefore, relative to the coordinate system centered on the mass standard datum and parallel to the coordinates of the MP instrument (i.e., the primed coordinates), the actual measured data in Table 3 show that the mean CG location in Position No. 1, with a 99\% confidence interval, lies within the intervals

$$
\begin{aligned}
& \eta_{X^{\prime}}=0.00308 \pm 0.00398 \mathrm{in} . \\
& \eta_{Y^{\prime}}=0.00068 \pm 0.00257 \mathrm{in} .
\end{aligned}
$$

Similarly, in Position No. 2 the mean CG location is found to lie within the intervals

$$
\begin{aligned}
& \eta_{X^{\prime}}=0.00553 \pm 0.00188 \mathrm{in} . \\
& \eta_{Z^{\prime}}=11.226 \pm 0.00110 \mathrm{in} .
\end{aligned}
$$

Upon discarding the $\mathrm{X}^{\prime}$ measurement in Position No. 2, as discussed in Section 8.4, the CG position for the mass standard, with a $99 \%$ confidence interval, is determined to lie within the rectangular prism region defined by

$$
\begin{aligned}
& \eta_{X^{\prime}}=0.00308 \pm 0.00398 \mathrm{in} . \\
& \eta_{Y^{\prime}}=0.00068 \pm 0.00257 \mathrm{in} . \\
& \eta_{Z^{\prime}}=11.226 \pm 0.00110 \mathrm{in} .
\end{aligned}
$$

It is emphasized that this interval represents only the statistical variation in the mass standard test data. For example, an adjustment for the tilt of the test article's axes still needs to be manually appended to express the CG offset relative to coordinates aligned to the mass standard (a different coordinate system from the primed coordinates, whose origin is simply offset from that of the MP instrument but whose axes are parallel to those of the MP instrument). Tilt parameters were shown in Table 2 and Figures 30 and 31 to be small from the metrological evaluation using the mass standard, viz., 0.00236 in. and $0.00101 \mathrm{in}$. for the $\mathrm{X}^{\prime \prime}$ and $\mathrm{Y}^{\prime \prime}$ dimensions, respectively. However, there could be some difference between how plumb the MMRTG mounts are as compared to the mass standard. Therefore, in lieu of providing a specific adjustment to the CG measurements for the MMRTG, a conservative tilt contribution of 0.003 in. to its overall CG position uncertainty has been assigned in Section 12. Other uncertainties, e.g., those that arise from temperature effects in the fixtures, are addressed and added to the total uncertainty in subsequent sections of this report. 


\subsection{Measurement Sensitivity}

Measurements described in the previous section with the mass standard established the overall accuracy capabilities of the MP testing system. Another informative check is to confirm the sensitivity of the measurement apparatus, i.e. is a small physical perturbation readily detectable. This was assessed by positioning a small, accurately known weight at an accurately known displacement from the MP instrument's rotational centerline in conjunction with the mass standard. One of the small, $3 / 4 \mathrm{in} /$ diameter fixture alignment pins described previously was used to make this assessment. By design, these pins provide a snug slip fit to the precision alignment holes in the base of the cradle permitting very accurate positioning. This feature makes these pins also very useful as special weights for making accurate analytical predictions of the sensitivity of the CG measurements. The exercise is described below.

First, a basic PART measurement was made with the mass standard in Position No. 1 (Run No. 1614). ${ }^{i}$ Without disturbing either the mass standard or the fixtures, the shortest alignment pin, having an accurately determined weight of $0.3319 \mathrm{lb}$., was placed in cradle base hole H located $9.9991 \mathrm{in}$. from the cradle centerline along the $+X$ axis, as shown in Figure 11. Another PART measurement was performed (Run No. 1615). The auxiliary pin was then sequentially moved to the other three cradle holes (all also nominally 10 in. from the cradle centerline and positioned as described below) then additional PART runs, No. 1616, 1617, and 1618 were performed. Those measurements are described subsequent to the discussion below for the initial measurement using cradle base hole $\mathrm{H}$.

The mass standard and auxiliary pin were removed, the mounting slides reset and secured, and the mounting screws and washers with the plastic standoffs were installed. A TARE measurement was made (Run No. 1619). This tare was applicable to the basic test and all four of the perturbed mass tests. The CG calculation (Run No. 1620) for the base case, using the PART Run No. 1614 with the common TARE run, was then performed. As shown in the calculation sheet, the mass standard net CG location along the $\mathrm{X}$ and $\mathrm{Y}$ axes was 0.0014 in. and 0.0028 in. respectively, as measured from the MP instrument's centerline.

For the slightly modified test article, now comprising the mass standard plus the auxiliary pin, the CG calculation (Run No. 1621) was performed using PART Run No. 1615, the measurement made with the auxiliary pin in hole $\mathrm{H}$. This calculation found the $\mathrm{X}$ and $\mathrm{Y}$ coordinates of the net $\mathrm{CG}$ to now be at +0.0356 in. and +0.0031 in. respectively, relative to the MP instrument's centerline. ${ }^{j}$

i Printouts from the MP software discussed in this section are provided in Appendix C, identified in the upper section by run number. The run number is a sequential number automatically assigned by the Space Electronics software to each operation. These printouts also serve as examples of the output from the software. The originals of these and the numerous other printouts generated during the course of the MP testing program for the MMRTG are kept at the MP testing station.

$\mathrm{j}$ The Y component of this measurement should not have changed. The $0.0003 \mathrm{in}$. (0.0031in. - 0.0028 in.) change measured may be a genuine small offset of the hole $\mathrm{H}$ centerline from the $\mathrm{X}$ axis or just an indication of the variation that occurred when remounting the mass standard. This and the other measurements described in the remainder of this section prompted the further inclusion of a conservative 0.002 -in positioning allowance in the overall uncertainty computation developed in Section 12.0 . 
By analysis, the net CG of the combined mass standard and auxiliary pin should have been located at a position determined by the dividing the total net moment $\mathrm{M}$ by the total net weight $\mathrm{W}$. Letting the subscripts MS and pin denote the mass standard and auxiliary pin respectively, the CG location along the $\mathrm{X}$ axis, denoted as $\mathrm{CG}_{\mathrm{X}}$, should now be located at

$$
\begin{aligned}
C G_{X} & =\frac{\sum M_{X}}{\sum W}=\frac{W_{M S} * X_{M S}+W_{p i n} * X_{p i n}}{W_{M S}+W_{p i n}} \\
& =\frac{(97.198 \mathrm{lb} .) *(+0.0014 \mathrm{in} .)+(0.3319 \mathrm{lb} .) *(+9.9991 \mathrm{in} .)}{97.198 \mathrm{lb} .+0.3319 \mathrm{lb} .} \\
& =+0.03542 \mathrm{in} .
\end{aligned}
$$

The discrepancy $\Delta$ between analytical prediction and measurement, rounded to four decimal places, is therefore

$$
\begin{aligned}
\Delta & =+0.0354 \mathrm{in} .-(+0.0356) \mathrm{in} . \\
& =-0.0002 \mathrm{in} .
\end{aligned}
$$

On a relative basis, the discrepancy is

$$
\Delta=\left|\frac{-0.0002 \text { in. }}{+0.03542 \text { in. }}\right|=0.0056=0.56 \%
$$

Placing the auxiliary pin into cradle base hole I on the $-\mathrm{X}$ axis similarly shows a predicted $\mathrm{CG}_{\mathrm{X}}$ of -0.03263 in. as compared to a measured $\mathrm{CG}_{\mathrm{X}}$ of -0.0331 in. (Run No. 1616 \& 1622). The discrepancy in this case is +0.0005 in. or $1.44 \%$.

Similar measurements were made with the auxiliary pin placed along the $\mathrm{Y}$ axis. Placing the auxiliary pin on the $+Y$ axis (hole $A$ ), the predicted $C_{G}$ was found to be 0.03682 in. while the measurement result (Runs 1617 and 1623) was 0.0368 in. indicating a discrepancy that rounds off to zero, within the four decimal places reported. For the $-\mathrm{Y}$ axis with the auxiliary pin in hole $\mathrm{G}$, the predicted $\mathrm{CG}_{\mathrm{Y}}$ is -0.03125 in. while the measurement showed -0.0316 in. (Runs 1618 and 1624) for a discrepancy of -0.0003 in. or $1.1 \%$.

The comparisons between measurements and the corresponding analytical predictions on both axes were all less than 0.0005 in. or $1.5 \%$. The purpose of these comparisons was only to establish that sufficiently sensitive measurements can be obtained with the new MP mounting equipment.

\subsection{Moment Measurements Using the Space Electronics Instrument}

The Space Electronics system includes a load cell that senses the offset moments along the instrument's two orthogonal axes in the horizontal plane. The mechanism is pre-loaded such that balanced loads register moments of approximately $2075 \mathrm{in}-\mathrm{lb}$. This is demonstrated in the balancing tests shown in Table 1, where the moments on all axes converge to approximately $2075 \mathrm{in}-1 \mathrm{~b}$ as the balance is improved. The preload could be subject to drift, however. The development below shows how this term can be negated, thereby avoiding drift errors. 
Net moments along both the positive and negative sides of a given axis produce forces that are sensed by the load cell. An off-center CG on the positive side of the $\mathrm{X}$ axis for example, will increase the moment on that side of the axis over and above the preload value, while the moment on the opposite side of the axis will correspondingly decrease relative to the preload. The preload moment is denoted by a zero subscript, viz., $\mathrm{M}_{0}$. The excess moment relative to the preload on either side of the axis is denoted as $\mathrm{M}_{\mathrm{ex}}$. Furthermore, the actual measured moment along the positive side of a given axis is denoted by $\mathrm{M}^{+}$and that on the negative side of the same axis by $\mathrm{M}^{-}$For an imbalance toward the positive axis, as in this example, equations for both measurements can be written using this terminology as

$$
\begin{aligned}
& M^{+}=M_{0}+M_{e x}^{+} \\
& M^{-}=M_{0}-M_{e x}^{-}
\end{aligned}
$$

Solving these equations simultaneously eliminates the preload, giving

$$
M_{e x}^{+}+M_{e x}^{-}=M^{+}-M^{-}
$$

Although the $\mathrm{M}_{\mathrm{ex}}$ terms are ostensibly equal, there could be a very small difference that results from any measurement errors. The best estimate, $\mathrm{M}_{\mathrm{BE}}$, of the true moment value relative to the preload is the arithmetic average of the relative moments,

$$
M_{B E}=\frac{M_{e x}^{+}+M_{e x}^{-}}{2}
$$

Upon combining these last two equations, the best estimate is related to the measurements themselves, as

$$
M_{B E}=\frac{M^{+}-M^{-}}{2}
$$

This moment pertains to whatever equipment is mounted. Two measurements are required to deduce the net moment for the test article alone. The first, the PART measurement, includes the test article, fixtures, and everything else that is mounted on the MP instrument when this measurement is performed. The second measurement, the TARE, is made with all of the same items mounted in exactly the same physical configuration except the actual test article has been removed. Each of these measurements yields a best estimated value per the above development. If a subscript $p$ denotes the PART measurements and a subscript $t$ denotes the TARE measurements, these equations become

$$
\begin{aligned}
& M_{B E-p}=\frac{M_{p}^{+}-M_{p}^{-}}{2} \\
& M_{B E-t}=\frac{M_{t}^{+}-M_{t}^{-}}{2}
\end{aligned}
$$


The difference between these two quantities is the net moment applicable to the test article alone, $\mathrm{M}_{\mathrm{TA}}$.

$$
M_{T A}=M_{B E-p}-M_{B E-t}
$$

Upon combining these,

$$
M_{T A}=\frac{M_{p}^{+}-M_{p}^{-}}{2}-\frac{M_{t}^{+}-M_{t}^{-}}{2}=\frac{1}{2} M_{p}^{+}-M_{p}^{-}-M_{t}^{+}+M_{t}^{-}-
$$

is derived.

The Space Electronics Instrument software automatically uses the user-specified PART and TARE moment measurements to perform these calculations without any user intervention. The development above is provided to support the consideration of temperature variation and the resulting measurement uncertainties in the results addressed in latter sections of this report where user intervention is needed.

\subsection{Test article Weight and CG Uncertainty}

After the net test article moment $\mathrm{M}_{\mathrm{TA}}$ has been measured, it must be divided by the user furnished testarticle weight to obtain the test article's CG offset along a given axis. With the weight denoted by W, the CG offset on the particular axis is therefore

$$
C G=\frac{M_{T A}}{W_{T A}}
$$

Since the test article weight is a user furnished parameter, an accurate CG offset calculation depends on an accurate determination of the test article weight. This is complicated further by the fact that the weighing of the MMRTG includes, out of handling and operational necessity, several equipment items that are not installed during the MP measurements. The net weight of the as-tested test article is found by subtracting the weight of these ancillary items to arrive at the as-tested weight (see Appendix J). Uncertainties in each of these terms affect the CG calculation uncertainty.

The gross weight of the test article, configured as weighed, is indicated by the subscript $g$ and the group of ancillary items removed thereafter, and absent during the MP measurement, is indicated by the subscript a. The net test article weight is therefore

$$
W_{T A}=W_{g}-W_{a}
$$

Uncertainty in the moment measurement is denoted $\delta \mathrm{M}$, and the uncertainty for each weight $\mathrm{W}$ is denoted $\delta \mathrm{W}$. These produce an uncertainty in the calculated CG by an amount $\delta \mathrm{CG}$ where, using the rules of differentiation,

$$
\delta C G=\delta\left[\frac{M_{T A}}{W_{T A}}\right]=\frac{\delta M_{T A}}{W_{T A}}-M_{T A} \frac{\delta W_{T A}}{W_{T A}^{2}}
$$


The uncertainties in the previously cited definitions of $\mathrm{M}_{\mathrm{TA}}$ and $\mathrm{W}_{\mathrm{TA}}$ are given by

$$
\delta M_{T A}=\frac{1}{2} \delta M_{p}^{+}-\delta M_{p}^{-}-\delta M_{t}^{+}+\delta M_{t}^{-}
$$

and

$$
\delta W_{T A}=\delta W_{g}-\delta W_{a}
$$

Combining these, the uncertainty in the CG calculation itself, that results from the uncertainties in the moments and weight measurements, becomes

$$
\delta C G=\frac{1}{2 W_{T A}} \delta M_{p}^{+}-\delta M_{p}^{-}-\delta M_{t}^{+}+\delta M_{t}^{-}-\frac{M_{T A}}{W_{T A}^{2}}\left(\delta W_{g}-\delta W_{a}^{-}\right.
$$

This equation comprises six independent terms in $\delta \mathrm{M}$ or $\delta \mathrm{W}$, each term of which can be either positive or negative. Multiple terms are usually combined in a square-root-of-the-sum-of-squares fashion to account for the low likelihood of all terms either stacking up in a common direction or fortuitously cancelling one another. Applying this approach, the estimated CG uncertainty is found to be

$$
\delta C G=\sqrt{\frac{1}{4 W_{T A}^{2}}} \boldsymbol{\$} M_{p}^{+^{2}}+\delta M_{p}^{-^{2}}+\delta M_{t}^{+^{2}}+\delta M_{t}^{-2}+\frac{M_{T A}^{2}}{W_{T A}^{4}} \mathbf{\phi} W_{g}{ }^{2}+\delta W_{a}^{2} \text { 一 } .
$$

For well balanced fixtures and test article relative to the particular axis being measured, the net moment $\mathrm{M}_{\mathrm{TA}}$ will be quite small and the square of this quantity causes the second term to rapidly become insignificant. This demonstrates the benefit of carefully balancing the fixtures and mounting the test article such that its CG is as close as practical to the center of rotation of the MP instrument. For such configurations, the CG uncertainty is controlled by the uncertainties in the individual moment measurements. This equation has been evaluated for several hypothetical situations parametrically in Table 4, which shows the intermediate results as well. Even for less well balanced configurations, when the uncertainties in the weights are small, the second term remains very small in comparison to the first term, as shown in Table 4. The design requirements specifications (Ref. 2) required that the mass of the MMRTG be known to within $0.5 \%$. For the approximately $100 \mathrm{lb}$. MMRTG, the allowable margin of error is $0.5 \mathrm{lb}$. The accuracy of the calibrated platform scale utilized for these tests is considerably better, always within $0.008 \mathrm{lb}$. and typically within $0.002 \mathrm{lb}$. The scale accuracy was checked using currently calibrated standards over the range $0-200 \mathrm{lb}$. prior to each official weighing. A record of the platform scale calibration check is provided in Appendix $\mathrm{H}$.

Conservative values for the moment uncertainties in the equation above can be gleaned from the repetitive runs listed in Table 5, showing

$$
\delta \mathrm{M} \approx 0.1 \mathrm{in}-\mathrm{lb}
$$

Assume a comfortably conservative value for the weight uncertainties of

$$
\delta \mathrm{W} \approx 0.01 \mathrm{lb} .(\max .)
$$


For a CG offset at the specified maximum radial limit of $5 \mathrm{~mm}(0.197 \mathrm{in}$.), the net moment for the approximately $100 \mathrm{lb}$. MMRTG would nominally be approximately $19.9 \mathrm{in}-\mathrm{lb}$. Parametric cases No. 1, 3, and 4 in Table 4 show that these would contribute less than a 0.001 in. uncertainty to the calculated CG position. This is probably the magnitude to be expected from moment and weighing measurement errors. This value has been included in the overall uncertainty shown in Section 12.1.

\subsection{Measurement Results}

Table 6 provides the results as reported by the MP instrument software. The table includes the measurement results for both the MMRTG and the four witnessed tests conducted with the mass standard. Since multiple tests were conducted with the mass standard, including the mounting/dismounting of the item, those results provide a reasonable basis for estimating the confidence intervals associated with all the test results. Since multiple tests were generally conducted, the run numbers of the PART and TARE data reports that were used for the calculations are shown in bold font. Reports for these calculations are provided in Appendix I. The results are shown relative to both the MP instrument and the user's coordinate system. The translation to the user coordinates is based on the user furnished metrological information discussed in Section 8.

The mean X, Y, Z coordinates of the CG position for the MMRTG in its test configuration, as calculated in Table 6, are listed below relative to its datum at the center of the mounting pads plane. Section 9.1 provides a complete explanation of the confidence interval evaluation.

$$
\begin{aligned}
& X=0.0968 \pm 0.0040 \mathrm{in} . \\
& Y=0.0276 \pm 0.0026 \mathrm{in} . \\
& Z=10.816 \pm 0.0011 \mathrm{in} .
\end{aligned}
$$

These offset values are the as-reported measurement results. The X and Y components of the CG coordinates reflect the non-symmetry in the MMRTG due to the presence of non-flight parts, such as the close-coupled Y-cable, bracket, cooling tube caps, etc., that were present during testing. Corrections for these items are to be made by the design agency, PWR.

Results from statistics calculations for the 99\% confidence interval specified in Reference 1 are also shown in Table 6 and summarized above. The confidence intervals cited represent a right prismatic region within which the CG is expected to lie with a $99 \%$ confidence interval as depicted in Figure 22 . These confidence intervals and the implied region were statistically determined from that data. In essence, the confidence intervals are based only on the observed variation inherent in the data. Systemic errors (biases), such as the induced tilt of the test article due to mountings, errors caused by expansion, or contraction from temperature variation that occurred between the PART and TARE measurements, are not reflected in these intervals. Uncertainties for such biases are added to the intervals shown above in Section 12, based on considerations discussed in this report to arrive at a total estimated uncertainty.

\section{TEMPERATURE CHANGES IN THE MOUNTING FIXTURES}

Test articles, such as radioisotope generators, whose temperatures differ markedly from that of typical room temperature have the potential to create temperature changes in the mounting fixtures. Such temperature changes can induce thermal expansions or contractions that, if sufficient in magnitude, effectively reposition both the fixtures and the test article. Repositioning affects both the calculated position of the CG relative to the MP instrument datum, as well as the translation of the CG position to the test article coordinates. This section quantifies these effects for the MMRTG testing. 


\subsection{General Thermal Considerations}

The temperatures in the fixtures were monitored to provide data to support analytical corrections to the MP measurements, if determined necessary, since very accurate mass properties are typically desired.

Temperature measurements were taken after the test article was mounted and the equipment was allowed at least two hours to reach stable thermal conditions. An accurate MP measurement requires that the TARE measurement be made with all items save the test article, in their exact same positions as during the PART measurement. Should the fixture temperatures during the TARE differ from the temperatures when the PART measurement was made, their resulting expansion or contraction could cause the calculation for the net test article moment to be in error. Because of the time required to dismount the test article and reconfigure the fixtures for the TARE, temperatures may well have changed.

The temperature phenomenon described above directly impacts the MP measurements relative to the MP instrument coordinates. Temperature variation can also affect the positional relationships between the test article datum and that of the MP instrument. The positional relationship, established by metrological investigation of the mounting fixtures at room temperature prior to operations with the test article, will be compromised by any fixture expansion or contraction. Such effects move the plane of the generator's mounting pads relative to the MP instrument's datum. This impacts the accurate translation of the CG and/or MOI measurements results from the MP instrument coordinate system to that of the test article.

In Position No. 1 the symmetry of the mounting fixtures and that of the MMRTG tend to negate the impact of any growth/contraction. By allowing the fixture temperatures to stabilize for a period of time after mounting the MMRTG in Position No. 1, the relative position of the MMRTG to the MP instrument rotational axis should not have changed in the horizontal plane. This is depicted in Figure 23. As indicated in the legend, all thermal growth is expected to be positive and symmetric, so that the only net movement of the test article's CG is upward from the MP instrument datum. Since the MP instrument only senses the mass properties in the horizontal plane, this thermal growth is of little concern for the CG measurement in this position. However, since MOI properties depend on the entire mass distribution, such measurements, if applicable, could very well be impacted.

In Position No. 2, longitudinal growth or contraction of the test article and its mounting fixtures is along the $\mathrm{Y}$ axis of the MP instrument, one of the coordinates that is sensed. In addition, non-symmetrical thermal effects occur. These are depicted in Figure 24. Fixture members physically above the generator will tend to be further heated by rising convective air currents while those members below the generator may begin to cool. The mounting fixtures are all fabricated from Type 6061-T651 (ASTM B209 and B211) aluminum, a material that has a relatively high coefficient of thermal expansion, at approximately $13.1 \mathrm{E}-6 \mathrm{~F}^{-1}$. The length of time required to reposition the generator and to perform new measurements limits the ability to quickly perform the measurement so as to minimize the time over which any thermal changes can occur.

The issues described above are in addition to whatever further considerations need to be made for the possibility that the temperature distribution in the test article during MP testing may differ from that during the mission application. This needs to be considered by the design agency based on the applicable conditions during the mission. Interestingly, the MMRTG itself can also be distorted by the nonsymmetrical temperatures induced in its shell while in a non-vertical position, such as Position No. 2. Vigorous convective cooling on the lower side, coupled with immersion in significantly heated air on the upper side, will cause the shell to bow slightly about a horizontal axis with the convex side upward. Deformation of the MMRTG may or may not be a concern to the mission application, since thermal induced buoyancy only occurs when immersed in a gaseous environment and in the presence of a gravitational field. Regardless, this phenomenon does not seriously impact the MP measurements. MP measurements in Position No. 2 are only used to obtain the longitudinal axis component of the CG and a slight axial bowing of the generator will not materially affect this value. 
Specific marks were made on the cradle, rotation fixture, and adapter ring to use as consistent targets for a direct reading contact-type temperature probe. Temperature readings at these locations were taken prior to and following the PART and TARE measurements. The intent was to either demonstrate negligible temperature changes or, if found not to be the case, to use these measurements to estimate the temperatures that prevailed as a basis to analytically correct the data. In the interest of minimizing operator radiation dose, not all stations were recorded when symmetry suggested only minor variation, e.g., side-to-side. In retrospect, additional measurements, e.g., on the upper members of the rotation fixture, would have been beneficial to the ultimate detailed analysis of the data.

\subsection{Evaluation of Fixture Temperature Variation}

As was developed in Section 9.4, the CG is located at a position measured from whatever datum the moment $\mathrm{M}_{\mathrm{TA}}$ is measured from, as

$$
C G=\frac{M_{T A}}{W_{T A}}
$$

It was further shown that to compute $\mathrm{M}_{\mathrm{TA}}$ the moments of the PART and TARE were required, where the positions of all items during the TARE were presumed identical to those during the PART measurement but without the test article present. Therefore, per the previous discussion, the question arises: suppose the fixture positions have, indeed, changed during the TARE for whatever reason, e.g. in the present investigation as a result of temperature changes in the fixtures. Then the TARE moment would no longer be $\mathrm{M}_{\mathrm{t}}$, but rather some different value denoted as $\mathrm{M}_{\mathrm{t}}^{\prime}$. Can the measurement be salvaged? The discussion in this section shows that it can be, and how this needs to be handled.

The test article moment is computed as was shown in Section 9.3 from the PART and TARE measurements as

$$
M_{T A}=\frac{M_{p}^{+}-M_{p}^{-}}{2}-\frac{M_{t}^{+}-M_{t}^{-}}{2}
$$

For brevity in the present discussion, this is written simply as

$$
M_{T A}=M_{p}-M_{t}
$$

$\mathrm{M}_{\mathrm{p}}$ and $\mathrm{M}_{\mathrm{t}}$ are understood to be contractions for their full definitions, as written before. Without changing the equation, the TARE moment term can be rewritten as

$$
M_{t}=M_{t}^{\prime}+M_{t}-M_{t}^{\prime}
$$

Upon inserting this into the previous equation,

$$
M_{T A}=\left(M_{p}-M_{t}^{\prime}\right)-\left(M_{t}-M_{t}^{\prime}\right)
$$


This can be recast into a CG form by dividing by the test article weight and rearranging the last term, giving

$$
C G_{T A}=\frac{M_{T A}}{W_{T A}}=\frac{\left(M_{p}-M_{t}^{\prime}\right)}{W_{T A}}-\frac{\left(M_{t}-M_{t}^{\prime}\right)}{W_{T A}}=C G_{T A}^{\prime}+\frac{\left(M_{t}^{\prime}-M_{t}\right)}{W_{T A}}
$$

The first term $\mathrm{CG}_{\mathrm{TA}}^{\prime}$ replaces its counterpart for the case where no displacement (here resulting from thermal expansion or contraction, but could be from any phenomenon) has occurred. The measurement software, being unaware that temperature effects may have occurred, only uses the actual PART and TARE measurement calculations, oblivious to any physical repositioning that has occurred to the hardware. The second term is the correction required to negate the error introduced by the CG displacement and thereby reestablish the validity of the test article CG calculation. This correction term must be manually appended to the $\mathrm{CG}_{\mathrm{TA}}^{\prime}$ value that is reported by the software.

The correction term can be computed once the displacements of all the components as occurred during the TARE have been evaluated. The total TARE moments are the summations of the individual moments among those components comprising the TARE. For example, the individual moment correction for the $\mathrm{j}^{\text {th }}$ component can be written as

$$
\left(M_{t}^{\prime}-M_{t}\right)_{j}=W_{j}^{*}\left(Y^{\prime}-Y\right)_{j}
$$

Defining $\left(\mathrm{Y}^{\prime}-\mathrm{Y}\right)_{\mathrm{j}}$ as $\Delta \mathrm{Y}_{\mathrm{j}}$, this equation can be written as

$$
\left(M_{t}^{\prime}-M_{t}\right)_{j}=W_{j}^{*} \Delta Y_{j}
$$

By invoking the thermal expansion coefficient, $\alpha_{j}(T)$, normally temperature dependent, for this $j^{\text {th }}$ component, each of the $\Delta \mathrm{Y}_{\mathrm{j}}$ terms can be evaluated as

$$
\Delta Y_{j}=\alpha_{j}(T) Y_{j} \Delta T_{j}
$$

The quantity $\Delta \mathrm{Y}_{\mathrm{j}}$ is the displacement (algebraic) that occurred to the individual $\mathrm{CG}$ of the $\mathrm{j}^{\text {th }}$ component (or $\mathrm{j}^{\text {th }}$ segment, if a large component had been divided into discrete segments) relative to its position during the PART measurement when the temperature of the component changed from its PART value $T$ to the TARE value T'. Calculation of the correction term becomes a matter of determining the CG displacements of each affected component or element, multiplying these displacements by their weights, summing these and dividing by the test article weight, yielding a final result for the overall CG of

$$
C G_{T A}=C G_{T A}^{\prime}+\frac{1}{W_{T A}} \sum_{j} W_{j} * \Delta Y_{j}
$$


This equation is evaluated and discussed in detail in Appendix E, taking into account the following observations from an inspection of the fixture temperature maps from the MMRTG test procedure (Ref. 3). These maps are provided in Appendix D. Temperature maps designated K-1 and K-2 respectively, show the temperatures measured on the cradle and on the rotation fixture with adapter ring as recorded just prior to the CG measurements. The cradle map K-1 shows there was only a very modest, approximately $2.5^{\circ} \mathrm{F}$ temperature increase in the cradle members from base upward toward the rotation fixture pivot point. The temperature increase in the higher elevation parts of the structure were the result of buoyancy induced convective heat transport. However, since these components were on the fringes of the convective flow patterns that developed around the generator, only these minimal temperature increases occurred. Furthermore, any symmetrical vertical growth or contractions in the cradle fixture will not affect the CG measurement.

The pre-test temperatures in the rotation fixture are all somewhat warmer than the local ambient temperature, which was not measured but, judging by the temperatures near the base of the cradle, was estimated to have been approximately $70^{\circ} \mathrm{F}$. The temperatures are a few degrees hotter near the bottom of the fixture and hotter still near the adapter ring. Since these measurements were made in Position No. 1, the temperature gradient indicates there was substantial heat conduction from the base of the generator into the adapter ring and rotation fixture base and then up into the upright members. This was in addition to any convective heat transport to the upright members.

Temperature map K-4 shows temperatures in the rotation fixture upon completion of the PART measurements and made as soon as the generator was returned to Position No. 1. Evidently, the continued conduction into the base of the fixture during the 75 min between pre- and post-test PART measurements and/or the distortion caused by being in Position No. 2 raised the temperatures in the rotation fixture about $2^{\circ} \mathrm{F}$ further and the temperature in the adapter ring by approximately $5^{\circ} \mathrm{F}$, although the temperature distribution is similar to that before the measurements. ${ }^{\mathrm{k}, \mathrm{l}}$ The local ambient temperature increase inside the testing cell may have also contributed. For ease of comparison, both the pre-test and post-test temperature distributions in the rotation fixture are plotted in Figure 25. As indicated in the figure, the temperatures included at the base and at the pivot point of the uprights (viz., the 0 . in. and $18.1 \mathrm{in}$. positions, respectively) are extrapolations from the limited measurements.

Following the CG testing, the generator was left in Position No. 1 overnight. This presumably allowed recovery of the temperature distributions that existed prior to the PART measurements. Immediately after dismounting the generator the next morning in preparation for TARE measurements, the temperatures in the fixtures were once more measured and recorded, as shown in temperature map K-6. The TARE in each position was performed, the rotation fixture returned to Position No. 1, and again the fixture temperatures were taken, as seen in temperature map K-8. The pre- and post-TARE temperature distributions are also plotted in Figure 25 for ease of comparison to the PART measurements. It is evident that some cooling occurred during the approximately one hour required to perform the TARE measurements in both positions.

${ }^{\mathrm{k}}$ The rotation fixture temperatures were only measured on the $-\mathrm{X}$ side. These members had been above the generator while in Position No. 2. This may have been at least partially responsible for the temperature increase.

1 This phenomenon had been anticipated. To hasten the return to Position No. 1 and in view of the typical consistency of the MP measurements, only a single PART measurement was performed on the MMRTG in Position No. 2. In Position No. 1 three measurements were made and the data from the most intermediate PART and TARE measurements were used in the calculations. 
Temperatures in the rotation fixture members just prior to the tares compared favorably to those prior to the testing performed the previous day, being generally just a fraction of a degree Fahrenheit warmer for corresponding measurement positions. Surprisingly, however, the temperatures recorded in the adapter ring are about $16^{\circ} \mathrm{F}$ cooler than those found previously (compare temperature maps K-4 and K-6). Note that the post-tare record, temperature map K-8, shows the adapter ring is now actually cooler than the adjacent members of the rotation fixture. Although this is not an impossible situation, it certainly indicates that the adapter ring cooled much faster than the adjacent members. This does not seem likely given the thick structure of the adapter ring. These observations cast some suspicion on the adapter ring temperature measurements and/or their record just prior to and following the tares. However, since no other data was available, the recorded data was used to conservatively estimate the fixture positions attributed to expansion/contraction, but an allowance was included in the reported overall uncertainty to reflect this and other similar secondary concerns.

The observed temperature changes that occurred between the PART and TARE measurements for Position No. 2 detailed calculations in Appendix E indicated that the required correction to the CG axial position was -0.0006 in. i.e. an adjustment of the composite CG toward the base of the MMRTG of 0.0006 in. This would normally be algebraically added to the axial CG position reported by the Space Electronics system. Given the small magnitude, an alternative treatment would be to assign an uncertainty contribution of $0.001 \mathrm{in}$. to the axial CG location to cover this modest temperature effect. This allowance is specifically identified in Section 12.

\section{COORDINATES TRANSLATION}

The measurement correction described in Section 10 refers to correcting the CG calculation that is reported by the Space Electronics software relative to the MP instrument datum should the TARE positions of the mounting equipment differ from their positions when the PART measurement was made. The CG position is also reported relative to the test article datum, since this is the datum ultimately of interest. The Space Electronics software computes this position by an algebraic translation of the CG position coordinates based on the user-furnished X and $\mathrm{Y}$ offsets between the MP instrument datum and the test article datum, as were determined by the metrology investigation described in Section 8 . This section discusses the translation of the measurement results and the consequences to the overall uncertainty.

The vertical offset of the test article's origin from the origin of the MP instrument was determined from design drawings of the mounting fixtures that controlled the actual dimensions when the fixtures were fabricated at room temperature. Figure 26 shows an adaptation of a view from INL Drawing 751208. The dimensions shown reflect the post-fabrication inspection. The as-built surfaces of the mounting slides on the Mk-II adapter ring are seen to be 11.096 in. from the rotation fixture pivot axis. When in Position No. 2, this is the Y (or Z, to the test article) offset between the MP instrument rotational centerline and the mounting surfaces for the test article on the Mk-II adapter ring. However, this asfabricated offset value needs to be corrected to the value that pertains to the temperatures that exist in both the rotation fixture and Mk-II adapter ring during the PART measurement. 
Upon mounting the MMRTG onto the fixtures and allowing the temperature transient to stabilize, the heat released caused all of the mounting fixtures to expand. Fortuitously, the elongation in the vertical members of the rotation fixture moved the base away from the pivots very nearly the same distance as the growth of the shorter, but hotter, adapter ring, moving the MMRTG mounting pads away from the rotation fixture base and nearer to the pivot. The net result was that during the PART measurement the position of the generator's mounting pads (the plane of the MMRTG datum) hardly moved at all. This can be seen in Spreadsheet E-1 (Table 7) from Appendix E by summing for both the adapter ring and the rotation fixture the applicable position entries (including only those that stack, which are shown in bold font) in the column labeled Thermal Growth or Shrinkage. ${ }^{\mathrm{m}}$ The upper surfaces of the adapter ring moved toward the pivot by $0.002246 \mathrm{in}$. while the upper surface of the rotation fixture base moved away from the pivot axis by $0.002446 \mathrm{in}$. Therefore, the net axial displacement of the tops of the mounting slides is seen to be away from the rotation fixture pivot by only $0.0002 \mathrm{in}$. This miniscule displacement of the tops of the adapter ring mounting slides showed that the room temperature offset of 11.096 in., when rounded, would still apply in this case. This is the value that is shown on the Space Electronics calculation reports for the Position No. 2 measurements.

During the TARE measurements, the growth of the fixtures was not balanced. Spreadsheet E-2 (Table 8) shows that (at least on the basis of the data recorded) the elongation of the rotation fixture arms was over twice the growth in the adapter ring height, causing a net displacement of $0.00127 \mathrm{in}$. at the tops of the adapter ring mounting slides away from the pivot. This is the same situation described in the previous section, i.e. where temperature changes occurred in the equipment causing their TARE positions to differ from their positions during the PART measurement. Section 10 provides the mathematical basis that allowed this phenomenon to be handled properly and the result reported there continues to apply.

\section{SUMMARY AND CONCLUSIONS}

The actual, as-tested CG position relative to the MMRTG datum at the center of a plane formed by the lower surface of the mounting pads was measured and found to be

$$
\begin{aligned}
& X=0.0968 \mathrm{in} . \\
& Y=0.0276 \mathrm{in} . \\
& Z=10.816 \mathrm{in} .
\end{aligned}
$$

These data must be adjusted for MMRTG applications having a different physical configuration and to correct for known errors in the physical position of the mounting equipment during the TARE measurement as compared to the equipment positions during the PART measurements. Potential random measurement errors manifest in uncertainties in the test article's CG position in all coordinates. This section summarizes the applicable adjustments required to these data and the individual uncertainty contributions from various testing considerations described in this report to arrive at the best estimate of the CG position and its overall confidence interval. Adjustments to the measured CG position for specific applications of the MMRTG to account for hardware configurations that may differ from the configuration when tested are the responsibility of the design agency.

m Spreadsheets from Appendix E have been assigned a sequential table number and are collected with other tables in this report. 
The non-random physical position errors were evaluated in this report using the methodology developed in Sections 10 and 11. However, because these were so small (a 0.0006 in. correction to the $Z$ component of the MMRTG CG position was calculated), it was decided instead to just include an allowance for this error in the uncertainties associated with the reported CG position.

\subsection{Uncertainties Summary}

\begin{tabular}{|c|c|c|}
\hline Basis & $\begin{array}{l}\text { Contribution } \\
\text { (in.) }\end{array}$ & Reference \\
\hline MP instrument moment statistical repeatability & $0.005^{*}$ & Table 6 \\
\hline Fixture re-positioning error & 0.002 & Footnote 9 \\
\hline Coordinates translation & 0.001 & Table 2 \\
\hline Tilt & 0.003 & Sections 8.4 and 9.1 \\
\hline Corrections for fixture temperature changes & 0.001 & Appendix E \\
\hline Moments/weight uncertainty effect in CG & 0.001 & Section $9.1 \&$ Table 4 \\
\hline Miscellaneous allowance & 0.005 & non-specific \\
\hline Total estimated absolute uncertainty & 0.018 in. & \\
\hline
\end{tabular}

\subsection{Conclusions}

The best estimated, corrected CG position for the MMRTG, configured as tested, was determined to lie at

$$
\begin{aligned}
& X=0.0968 \mathrm{in} . \\
& Y=0.0276 \mathrm{in} . \\
& Z=10.816 \mathrm{in} .
\end{aligned}
$$

This point lies in the center of a spherical region having, with $99 \%$ certainty, a radius of 0.018 in. Thus, the estimated total uncertainty is comfortably enveloped within the PWR specified spherical uncertainty criterion of $10 \mathrm{~mm}(0.394 \mathrm{in}$.).

\section{REFERENCES}

1. PWR document No. EID-09038, Mars Science Laboratory Radioisotope Thermoelectric Generator Test Plan / Requirements

2. PWR Specification No. RJ00554, Design Requirements [for the Mars Science Laboratory MultiMission Radioisotope Thermoelectric Generator]

3. INL test procedure, RPS/HS-OI-28220, MMRTG Mass Properties Test

4. INL test procedure, SSPSF-OI-28200, Mass Properties Testing Station Operation

5. American Society for Metals, Metals Handbook $9^{\text {th }}$ Ed., Vol. 2, Properties and Selection: Nonferrous Alloys and Pure Metals, pp. 115-116.

6. J. S. Hunter, Design of Experiments, Westinghouse Learning Corporation (1966). 


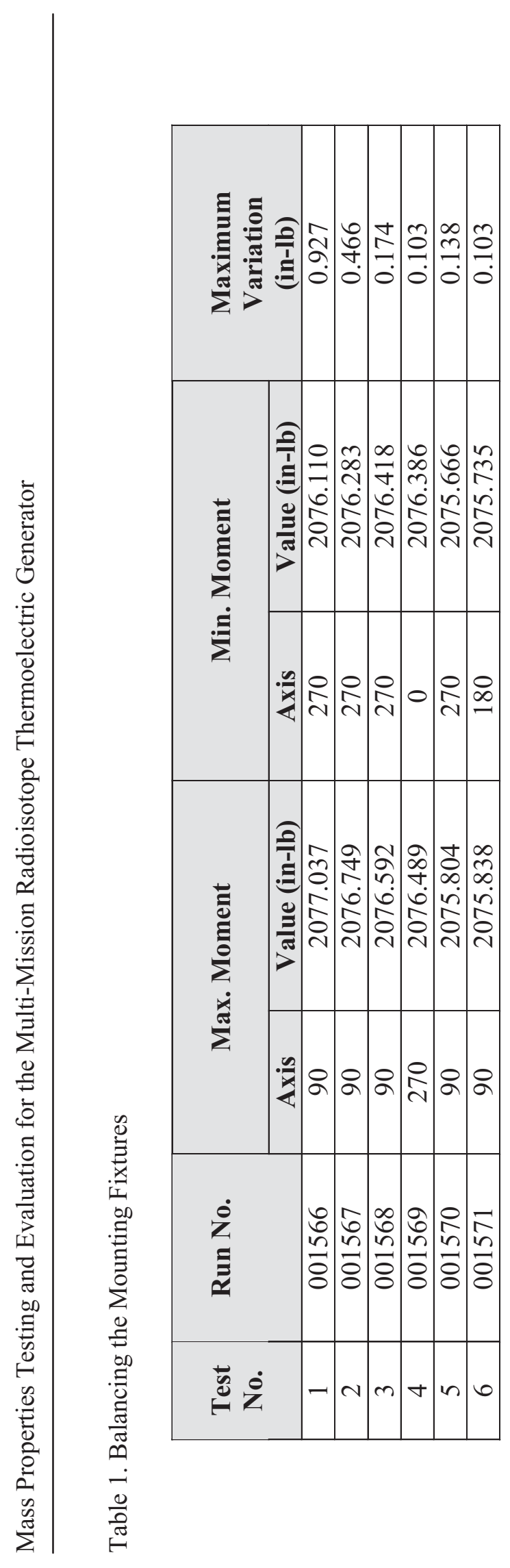


Table 2. Metrology Summary for the Mass Standard

\begin{tabular}{|c|c|c|c|}
\hline Parameter & Definition (see Figures 27-31) & Value* & Units \\
\hline$\Phi$ & $\begin{array}{l}\text { Angle of the projection onto the } \mathrm{X}-\mathrm{Z} \text { plane, measured from the } \mathrm{Z}^{\prime} \\
\text { axis }\end{array}$ & 0.0046 & degrees \\
\hline$\theta$ & Actual angle, measured from the $Z^{\prime}$ axis & 0.0052 & degrees \\
\hline$\xi$ & $\begin{array}{l}\text { Angle of the projection on the } \mathrm{X}-\mathrm{Y} \text { plane, measured from the } \mathrm{X}^{\prime} \\
\text { axis }\end{array}$ & 27.92 & degrees \\
\hline$\beta$ & $\begin{array}{l}\text { Angle of the projection onto the } Y-Z \text { plane, measured from the } Z^{\prime} \\
\text { axis }\end{array}$ & 0.0024 & degrees \\
\hline $\mathrm{X}_{0}$ & $\begin{array}{l}\text { Offset of the test article origin from that of the MP instrument } \\
\text { along the } \mathrm{X} \text { axis }\end{array}$ & -0.00273 & inch \\
\hline $\mathrm{Y}_{0}$ & $\begin{array}{l}\text { Offset of the test article origin from that of the MP instrument } \\
\text { along the Y axis }\end{array}$ & 0.00276 & inch \\
\hline $\mathrm{Z}_{0}$ & $\begin{array}{l}\text { Offset of the test article origin from that of the MP instrument } \\
\text { along the instrument's } Z \text { axis }\end{array}$ & 14.904 & inch \\
\hline $\mathrm{X}_{\mathrm{CG}}$ & $\begin{array}{l}\text { Measured position of the } \mathrm{CG} \text { as measured on the MP instrument } \\
\mathrm{X} \text { axis }\end{array}$ & 0.00053 & inch \\
\hline $\mathrm{Y}_{\mathrm{CG}}$ & $\begin{array}{l}\text { Measured position of the CG as measured on the MP instrument } \\
\text { Y axis }\end{array}$ & 0.00333 & inch \\
\hline $\mathrm{Z}_{\mathrm{CG}}$ & $\begin{array}{l}\text { Computed position of the CG as measured on the MP } \\
\text { instrument's } Z \text { axis }\end{array}$ & 26.134 & inch \\
\hline $\mathrm{X}_{\mathrm{CG}}^{\prime}$ & $\begin{array}{l}\text { Computed position of the } \mathrm{CG} \text { as measured on the } \mathrm{X}^{\prime} \text { axis of the } \\
\text { mounting fixtures, } \mathrm{X}_{\mathrm{CG}}^{\prime}=\mathrm{X}_{\mathrm{CG}}-\mathrm{X}_{0}\end{array}$ & 0.00326 & inch \\
\hline $\mathrm{Y}_{\mathrm{CG}}^{\prime}$ & $\begin{array}{l}\text { Computed position of the } \mathrm{CG} \text { as measured on the } \mathrm{Y}^{\prime} \text { axis of the } \\
\text { mounting fixtures, } \mathrm{Y}_{\mathrm{CG}}^{\prime}=\mathrm{Y}_{\mathrm{CG}}-\mathrm{Y}_{0}\end{array}$ & 0.00195 & inch \\
\hline$Z_{\mathrm{CG}}^{\prime}$ & $\begin{array}{l}\text { Computed position of the } \mathrm{CG} \text { as measured on the } \mathrm{Z}^{\prime} \text { axis of the } \\
\text { rotation fixture }\end{array}$ & 11.226 & inch \\
\hline $\mathrm{X}_{\mathrm{CG}}^{\prime \prime}$ & $\begin{array}{l}\text { Computed position of the CG as measured on the } \mathrm{X}^{\prime \prime} \text { axis of the } \\
\text { test article }\end{array}$ & 0.00236 & inch \\
\hline $\mathrm{Y}_{\mathrm{CG}}^{\prime \prime}$ & $\begin{array}{l}\text { Computed position of the CG as measured on the } \mathrm{Y}^{\prime \prime} \text { axis of the } \\
\text { test article }\end{array}$ & 0.00101 & inch \\
\hline$Z^{\prime \prime}{ }_{C G}$ & $\begin{array}{l}\text { Computed position of the } C G \text { as measured on the } Z^{\prime \prime} \text { axis of the } \\
\text { test article }\end{array}$ & 11.226 & inch \\
\hline
\end{tabular}

* Values are best-estimated results of multiple measurements. 


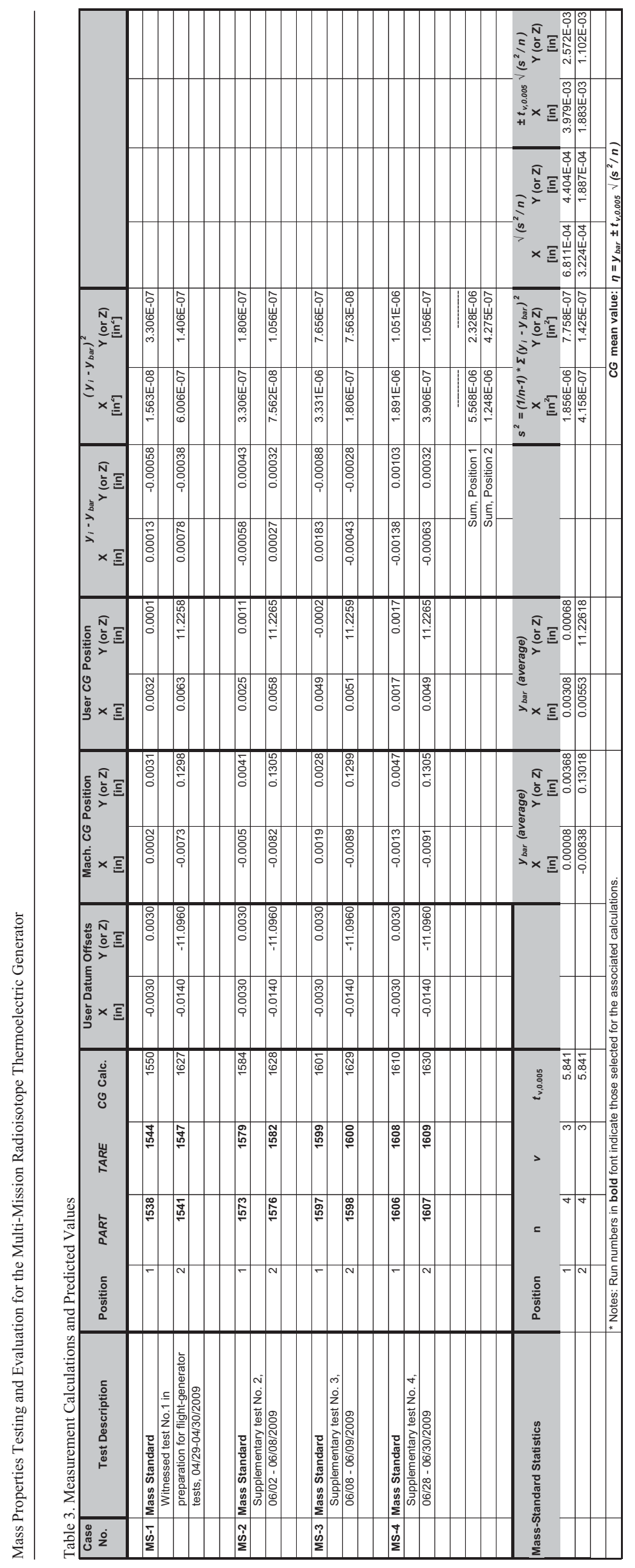




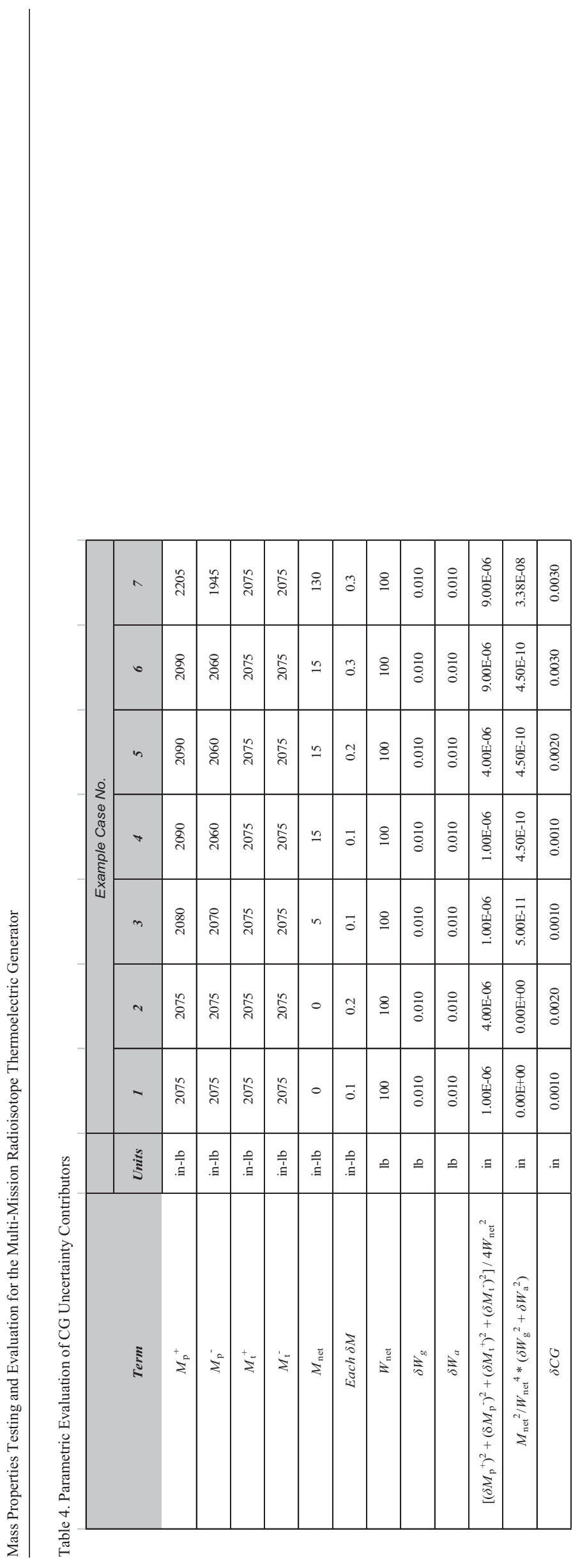




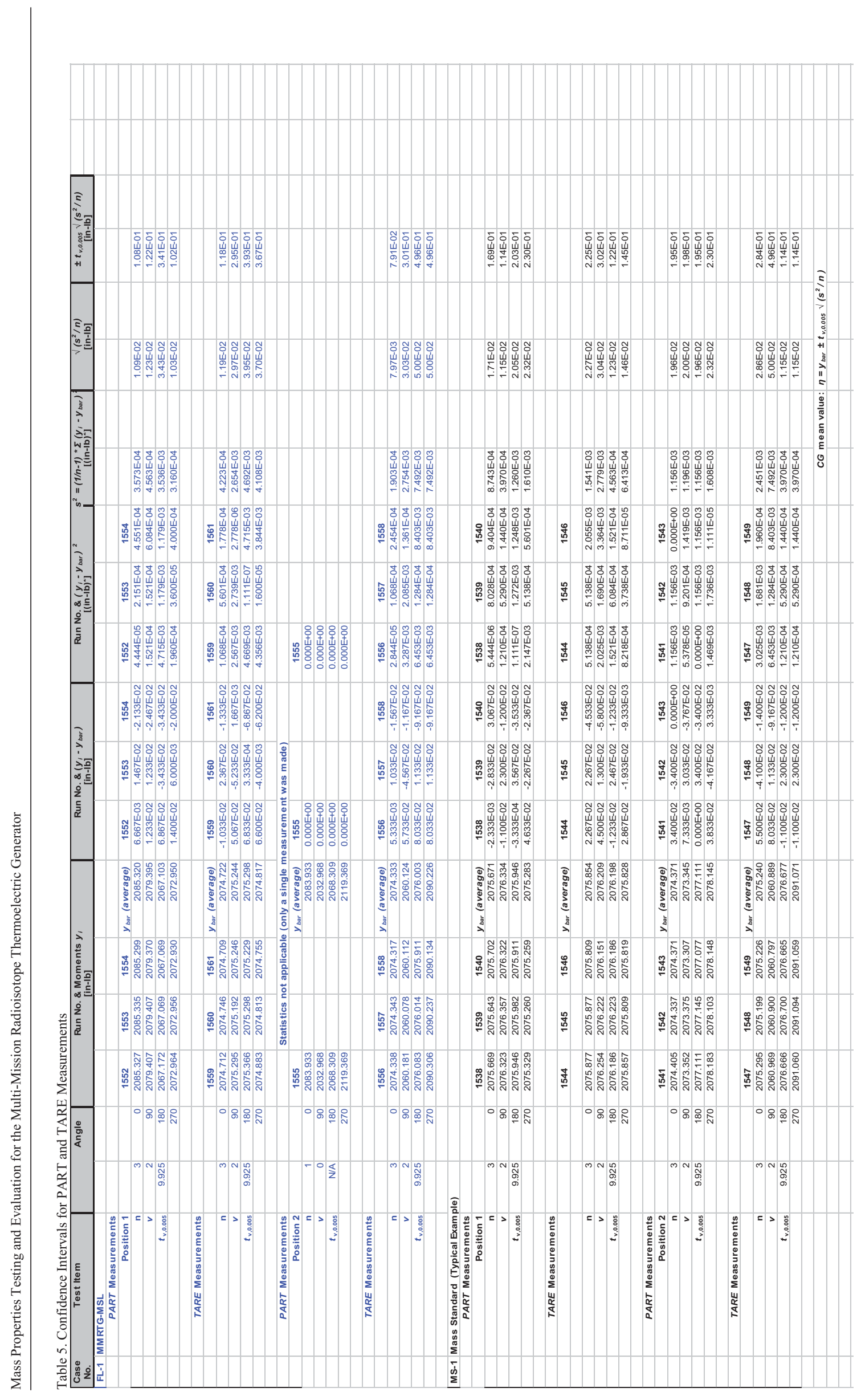




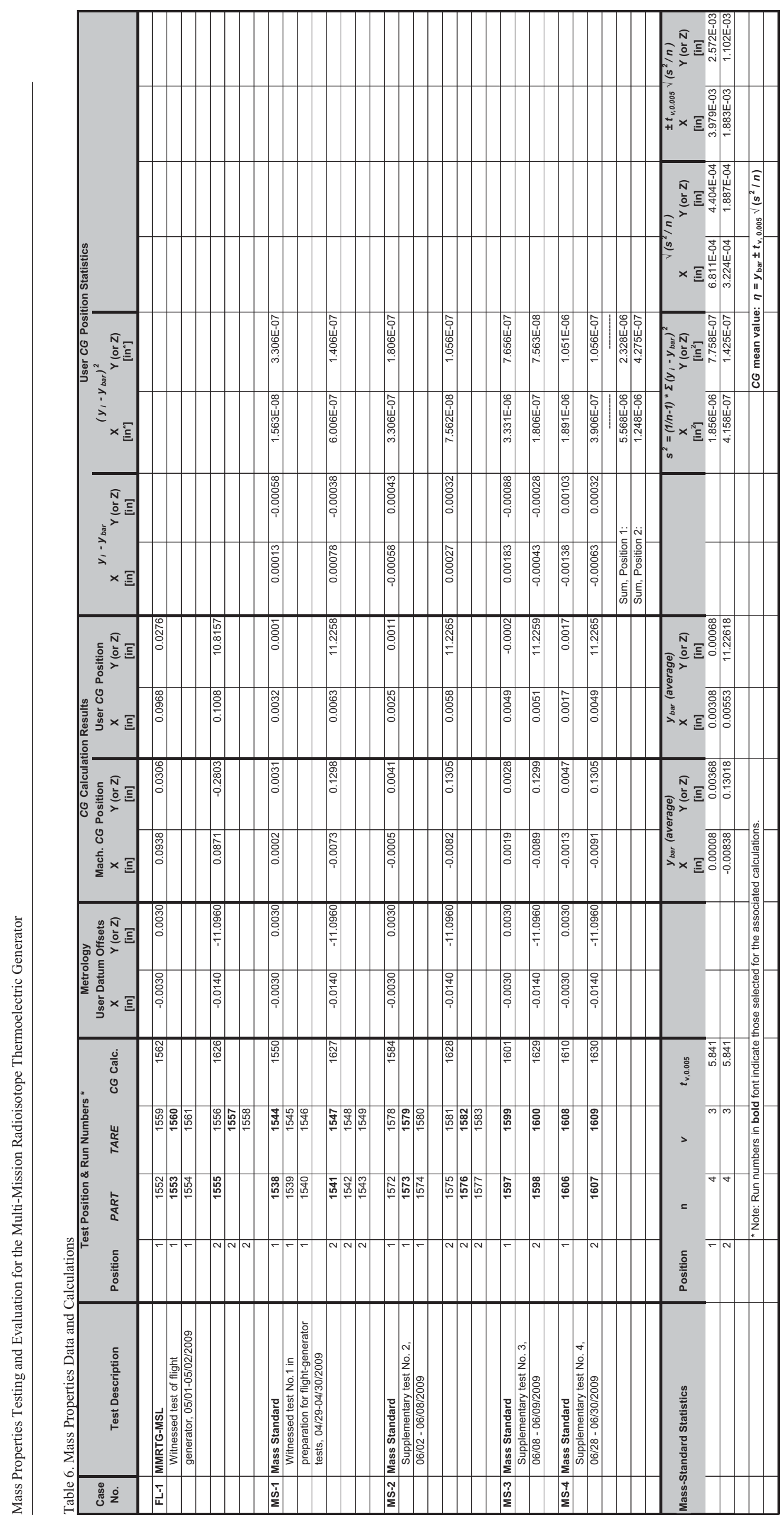




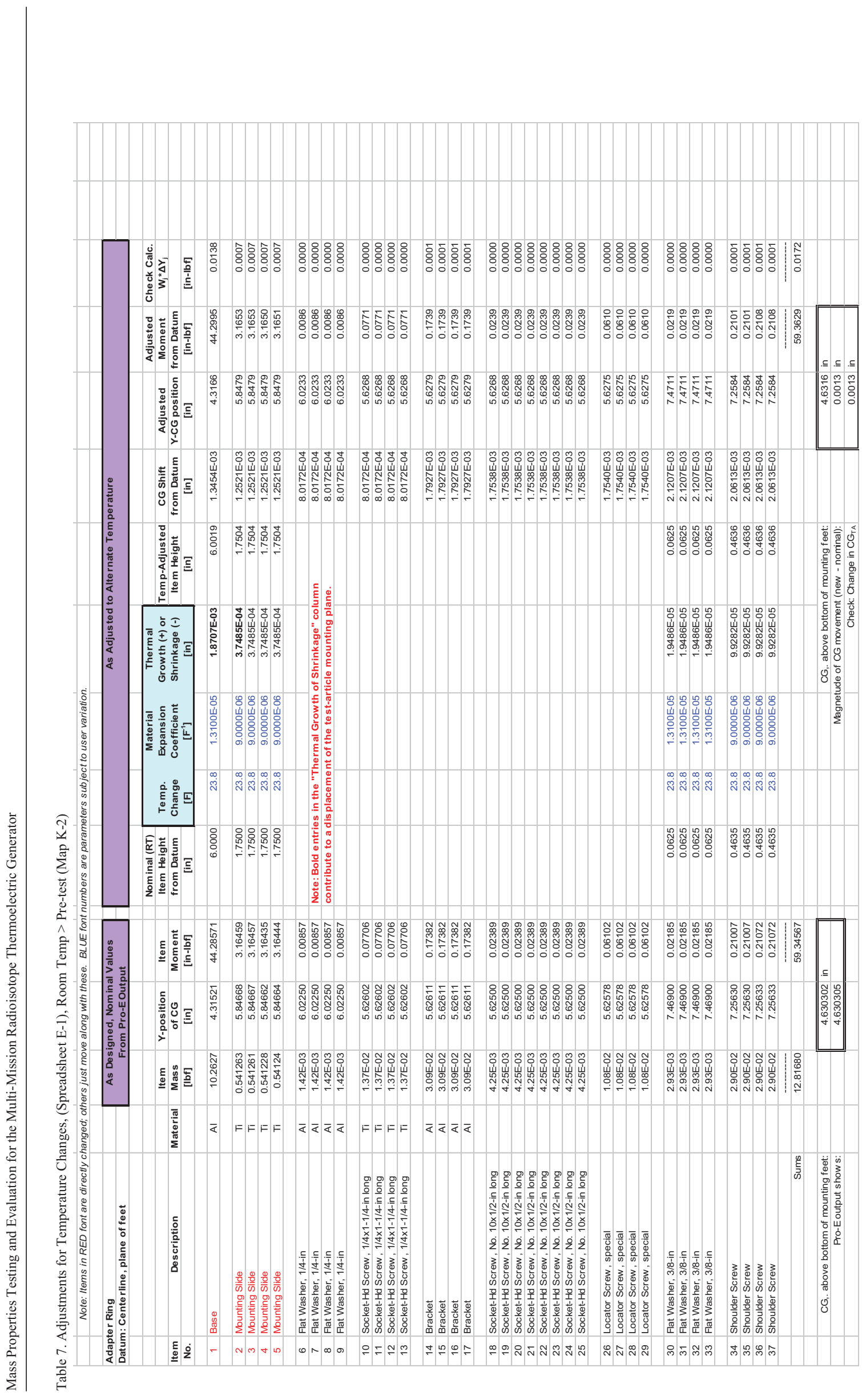




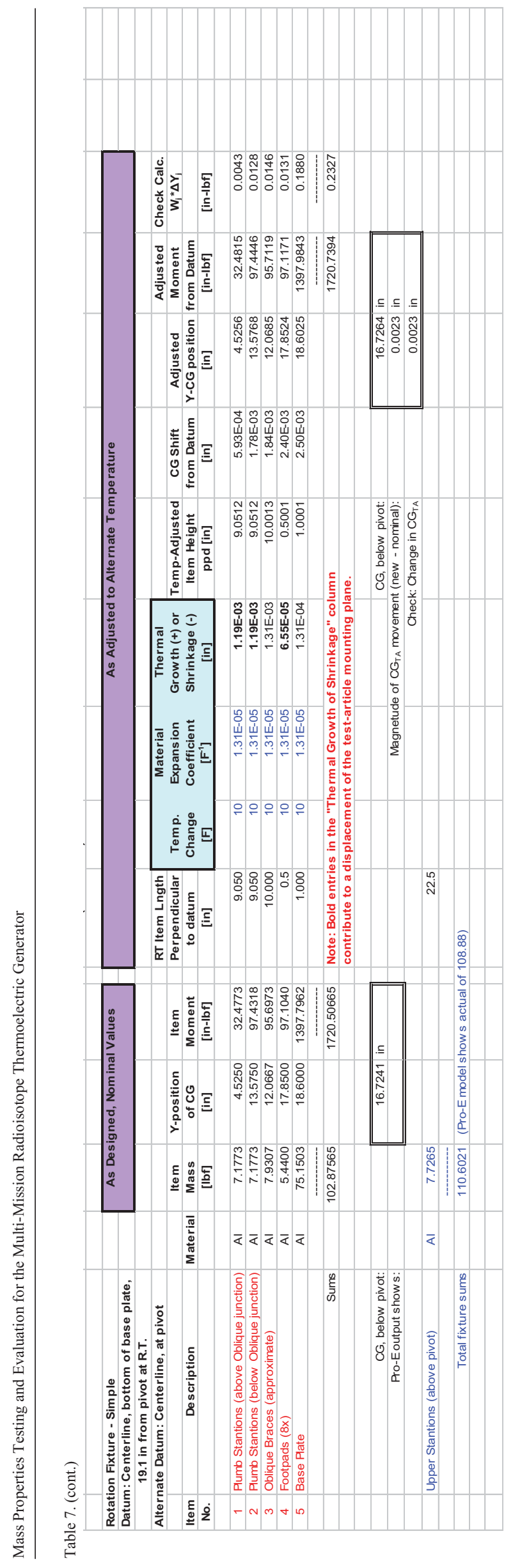




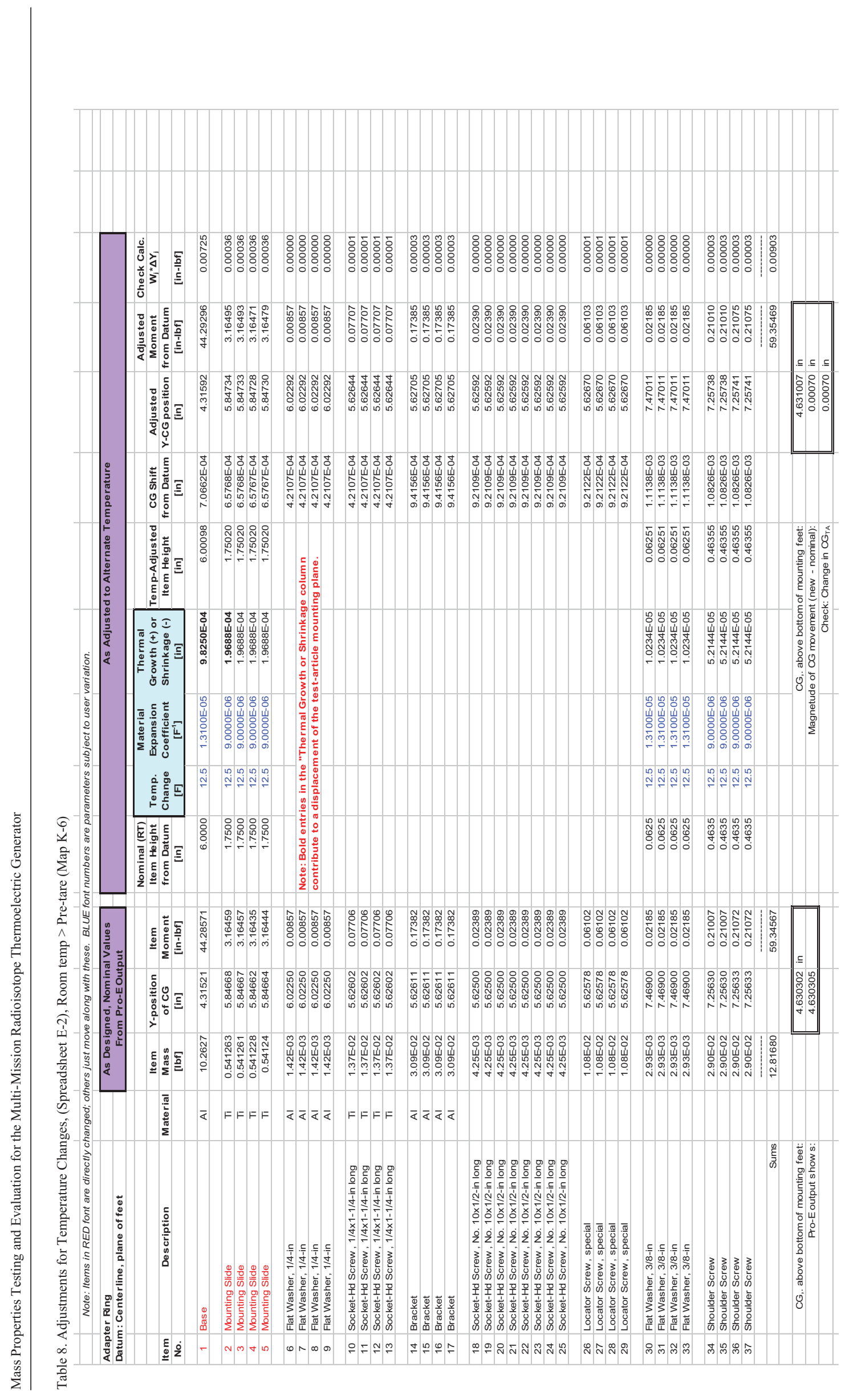




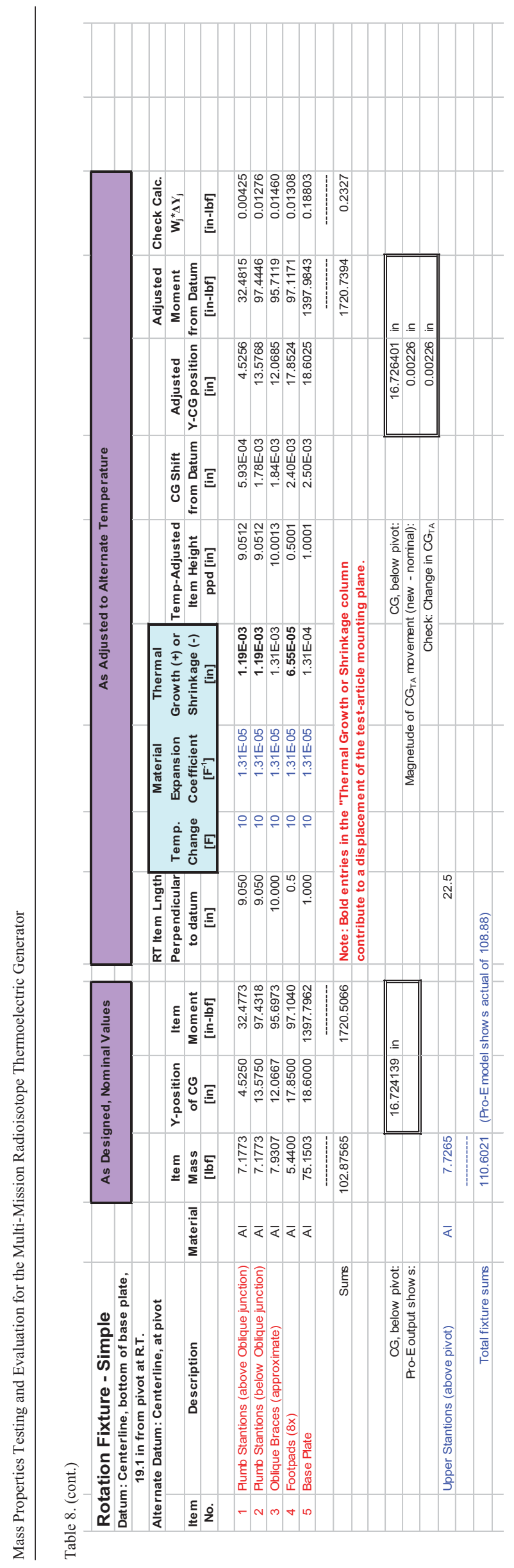


Table 9. Spreadsheet B-1 - Y Offset Constructions Characteristics

\begin{tabular}{|c|c|c|c|}
\hline \multirow{2}{*}{$\begin{array}{c}\text { View } \\
\text { (cf., Fig. 14) }\end{array}$} & $\begin{array}{c}\text { Cradle-Pin } \\
\text { Position }\end{array}$ & $\begin{array}{c}\boldsymbol{\Delta} \text { Y Direction (cf., App. B) } \\
\text { Ambiguous }\end{array}$ & $\begin{array}{c}\boldsymbol{Y} \text {-axis } \\
\text { Not Ambiguous }\end{array}$ \\
\cline { 3 - 4 } & Near $(+), 4(-)$ & Red & Black \\
\hline $2(+), 4(-)$ & Away & Black & Red \\
\hline $1(-), 3(+)$ & Near & Black & Red \\
\hline $1(-), 3(+)$ & Away & Red & Black \\
\hline
\end{tabular}




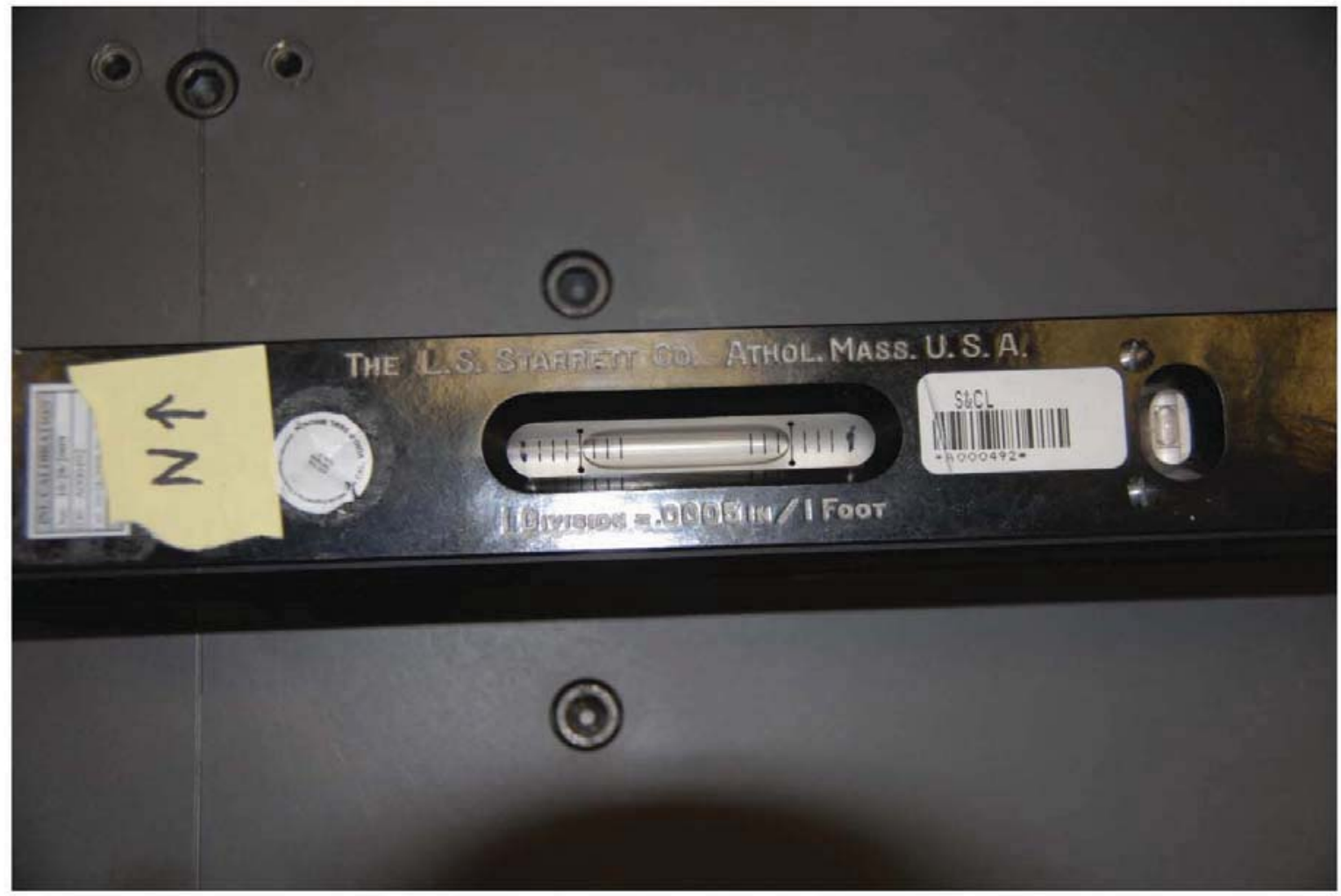

Figure 1. Leveling the MP instrument

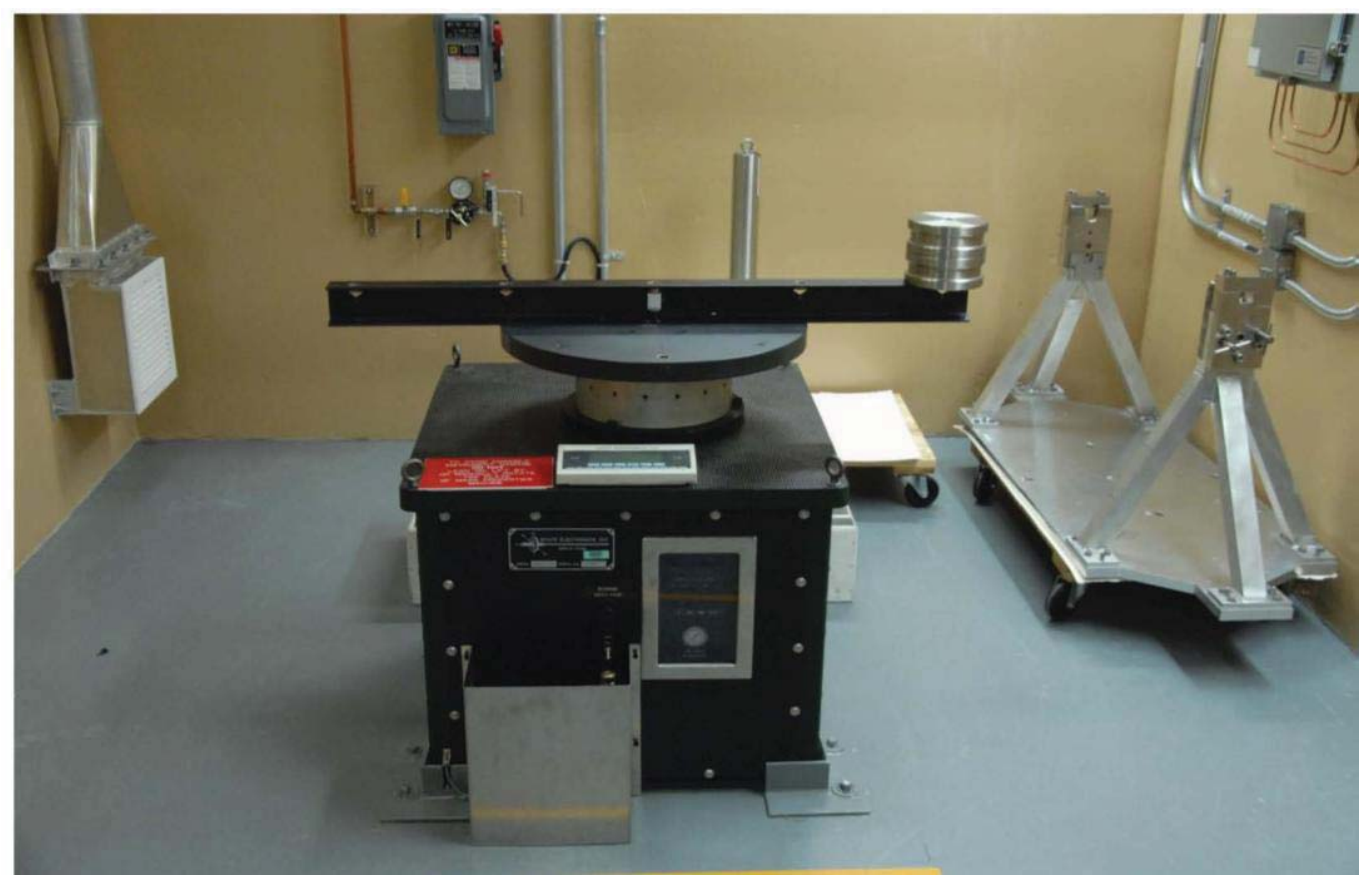

Figure 2. Equipment setup for calibrating the MP instrument 


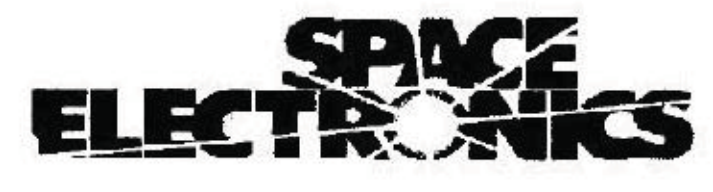

Model : KSR1320-1500

' Version: $\mathbf{4 . 2 . 3}$

Serial : $2938 / 72818$
RunNumber : 001603

Date : June 26, 2009

Time: 11:24

Operator : Chris Browning

\section{CG Calibration Results}

\section{Readings}

\begin{tabular}{|c|r|rrr|r|} 
& $\begin{array}{r}\text { Step 1 Readings } \\
\text { (counts) }\end{array}$ & $\begin{array}{c}\text { Step 1 Angles } \\
\text { (deg) }\end{array}$ & $\begin{array}{c}\text { Step 2 Readings } \\
\text { (counts) }\end{array}$ & $\begin{array}{c}\text { Step 2 Angles } \\
\text { (deg) }\end{array}$ \\
\hline Reading Position 1: & $\mathbf{8 4 2 5 9 . 0}$ & 0.000 & 36810.2 & 0.000 \\
\hline Reading Position 2: & 60468.0 & & 90.000 & 60616.1 & 90.000 \\
\hline Reading Position 3: & 36843.1 & & 180.000 & 84285.9 & 180.000 \\
\hline Reading Position 4: & 60656.9 & 270.000 & 60488.3 & 270.000 \\
\hline
\end{tabular}

\section{Calculated Calibration Values}

Excessive Variation: FALSE

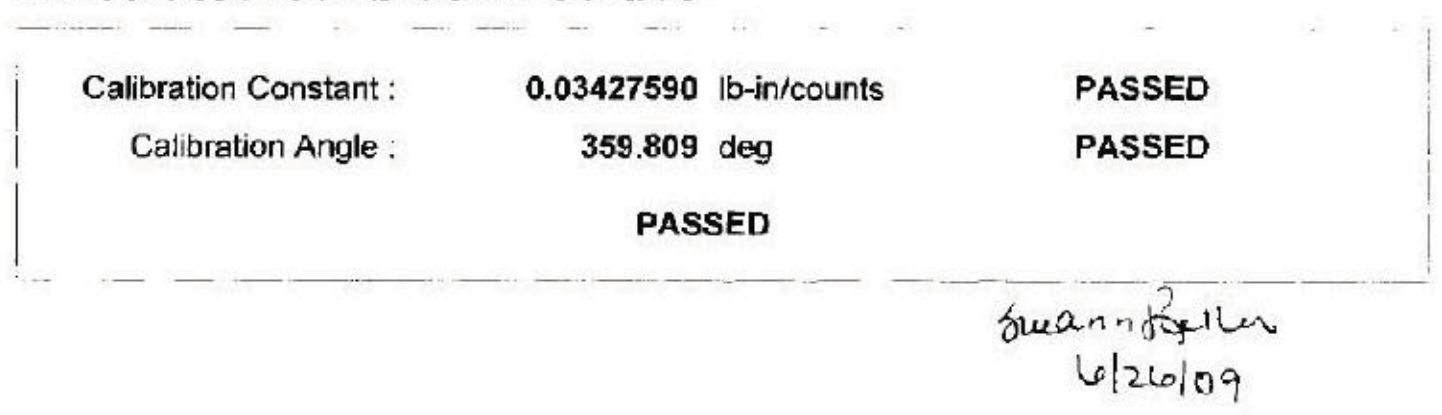

Figure 3. MP instrument calibration report 

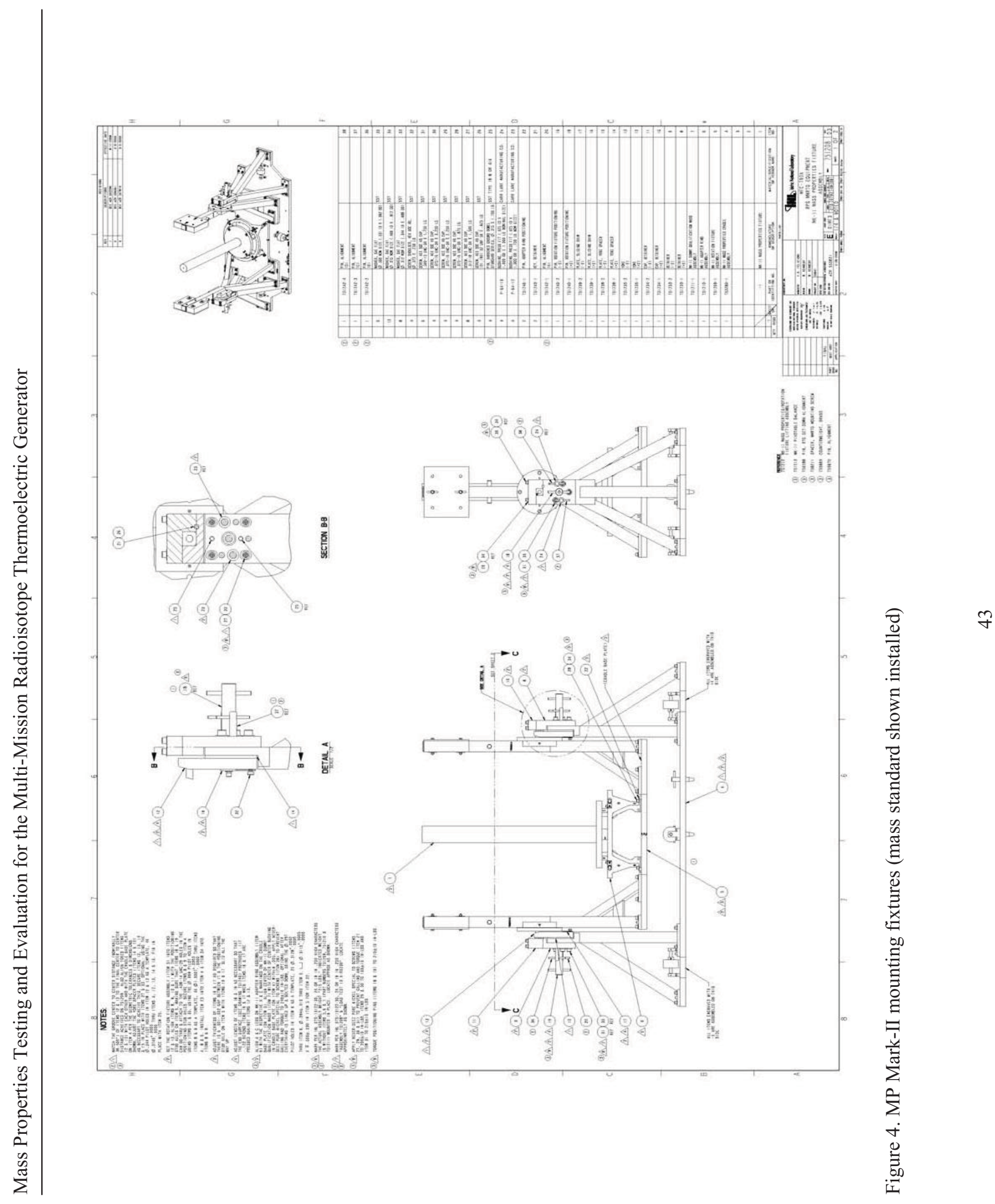


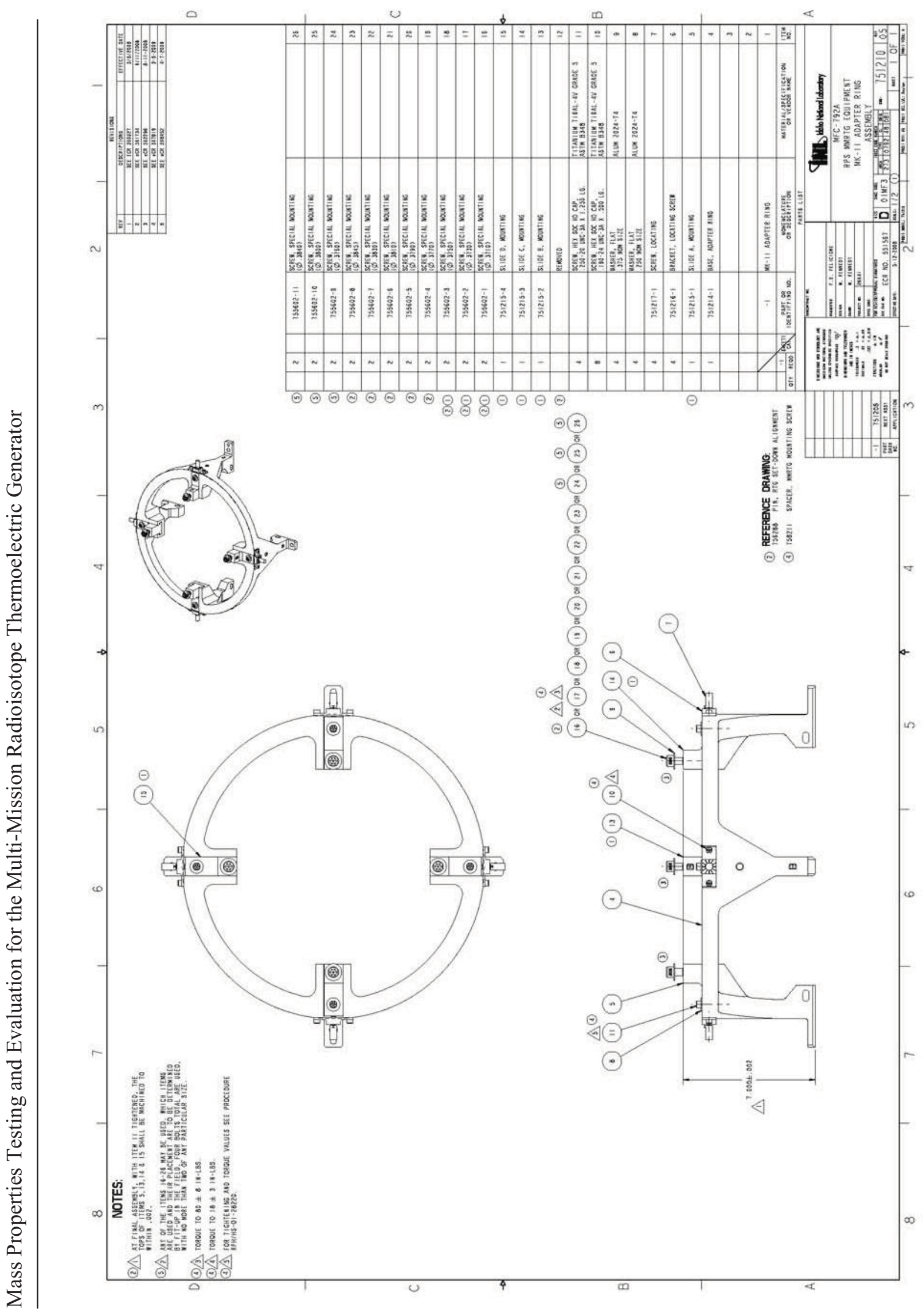




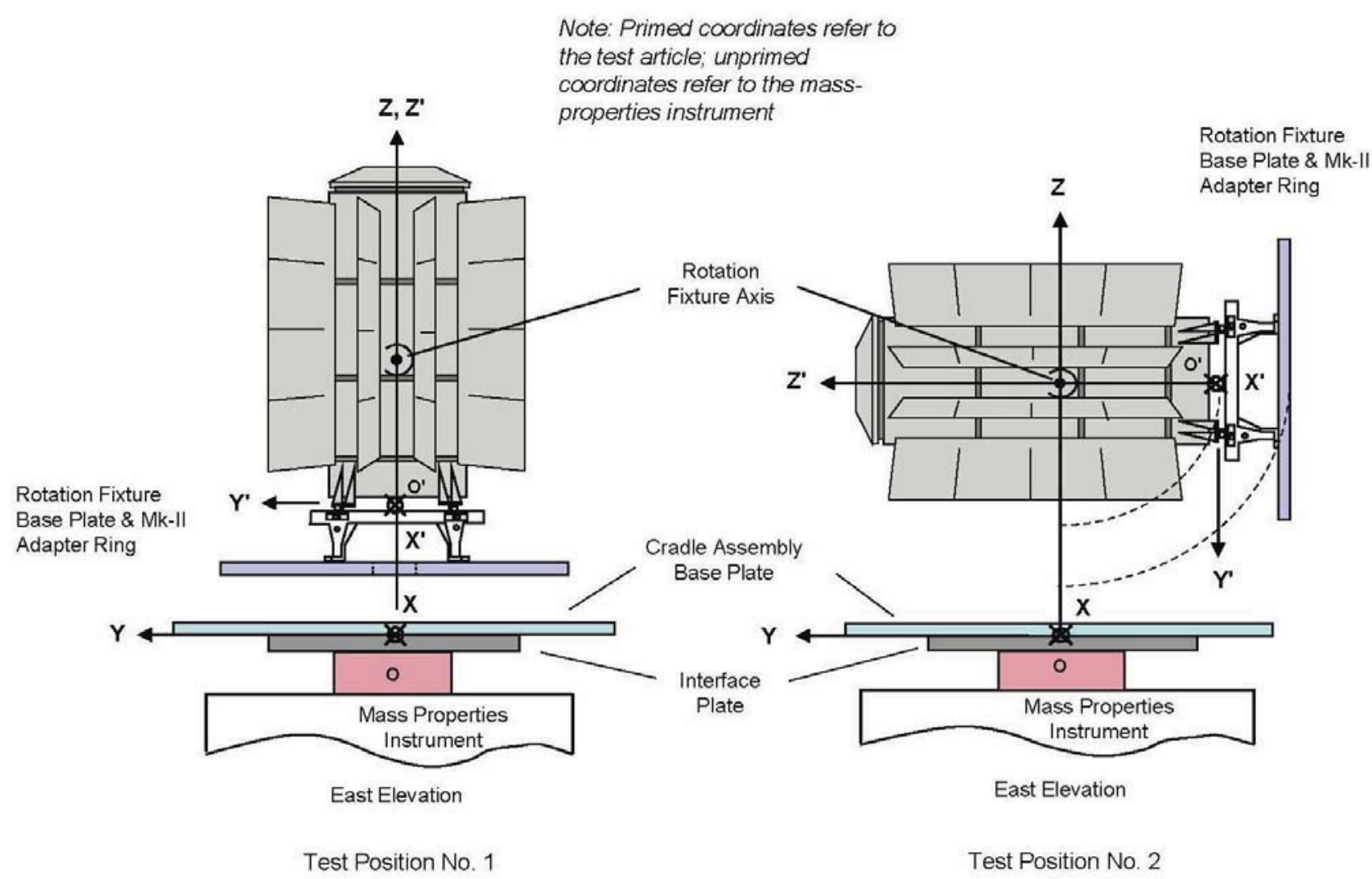

Figure 6. Coordinate systems and test positions 


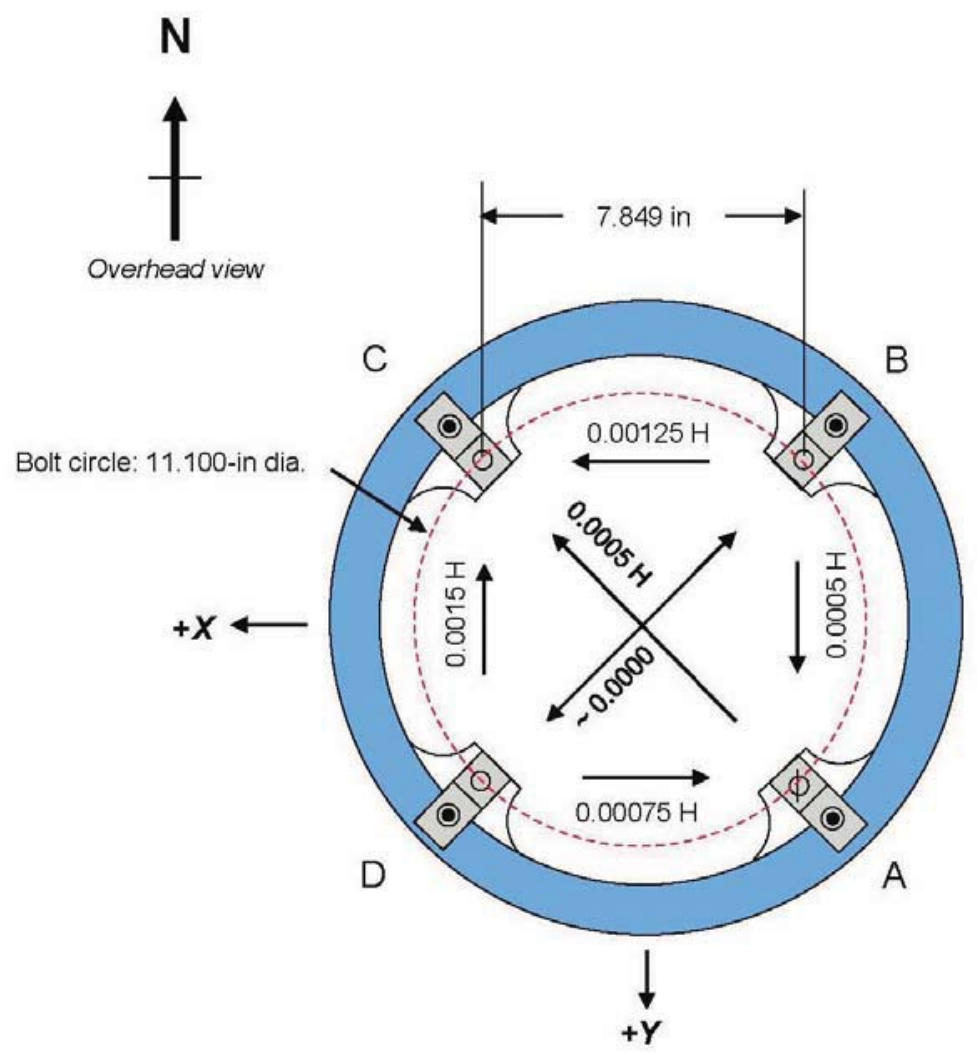

Measurement Equipment:

Type: Starrett Precision Level, 0.0005 inft/div

ID Number: $\underline{\mathrm{S} / \mathrm{N} A 0661}$

Calibration Due Date: 08 June 2010

Measurement Date: $\underline{02}$ July 2009

Remarks: Level placed on top of adjacent \& diagonal mounting slides; arrowheads denote the high side $(H)$ of the level. Measurements indicated are in in $/ f t$, as read on instrument.

Figure 7. Final level checking of the MP mounting fixtures 


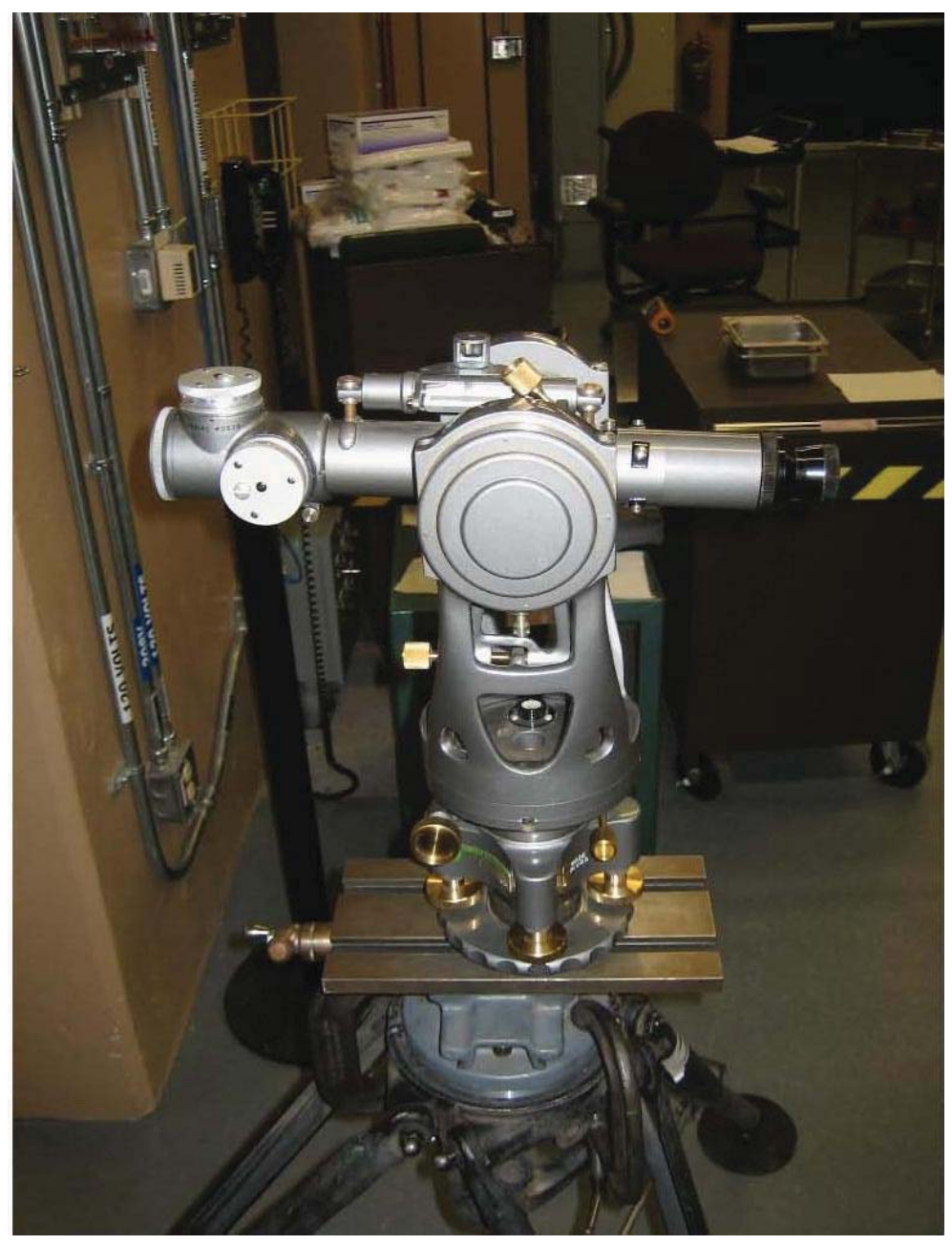

Figure 8. Precision transit setup for alignment determination 


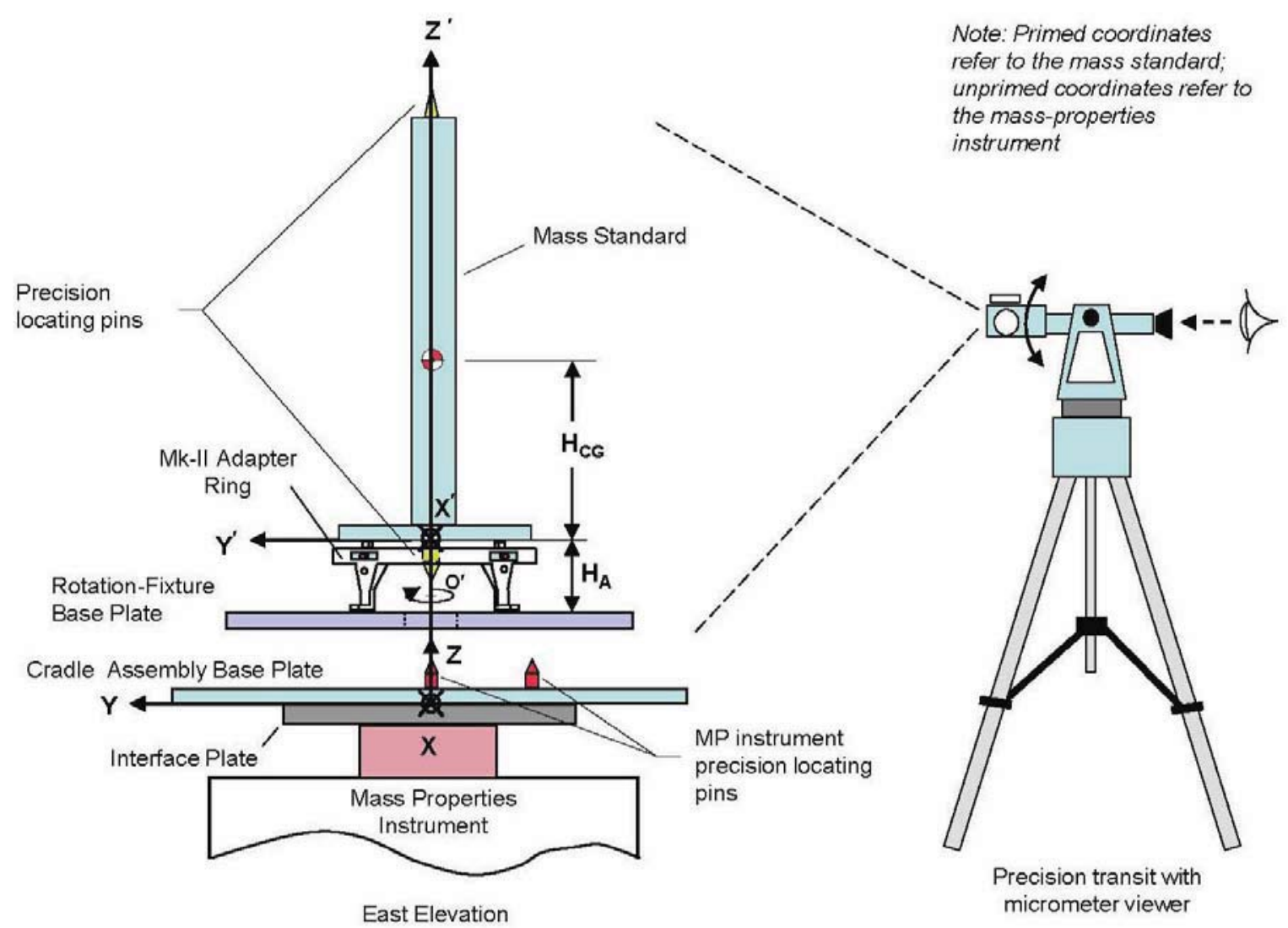

Figure 9. Determining offsets of coordinate systems

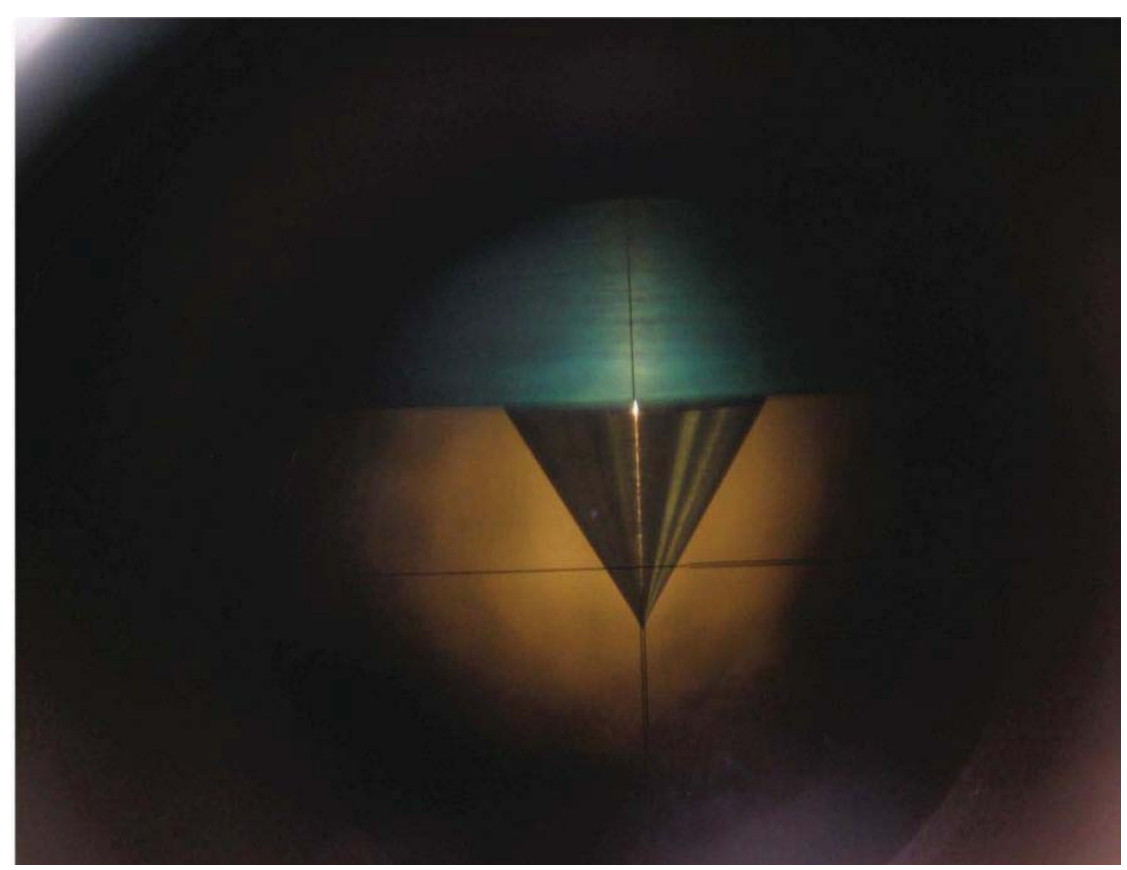

Figure 10. Typical view of lower alignment pin through precision transit 


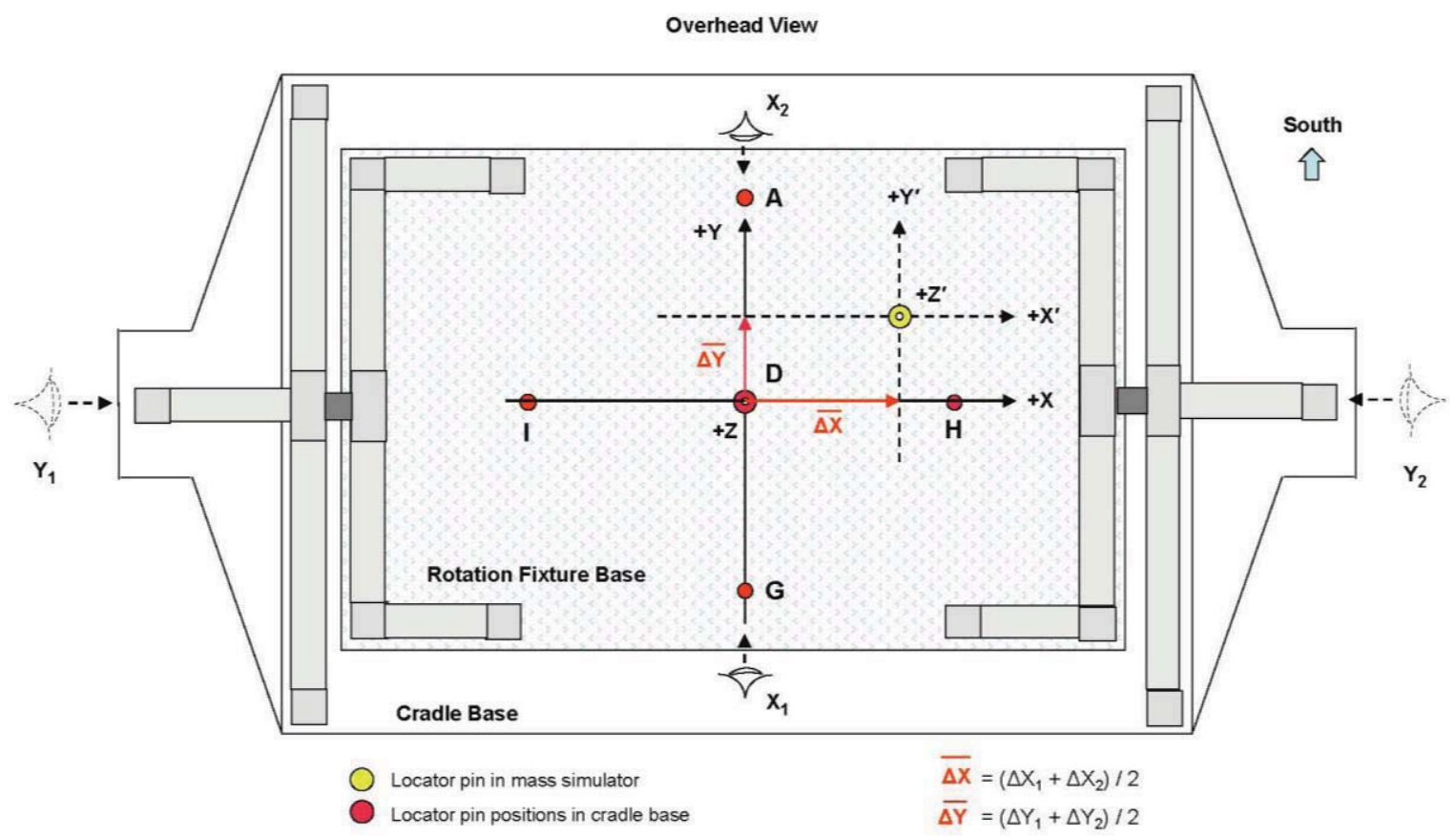

Figure 11. Determining offsets of test article relative to MP instrument 


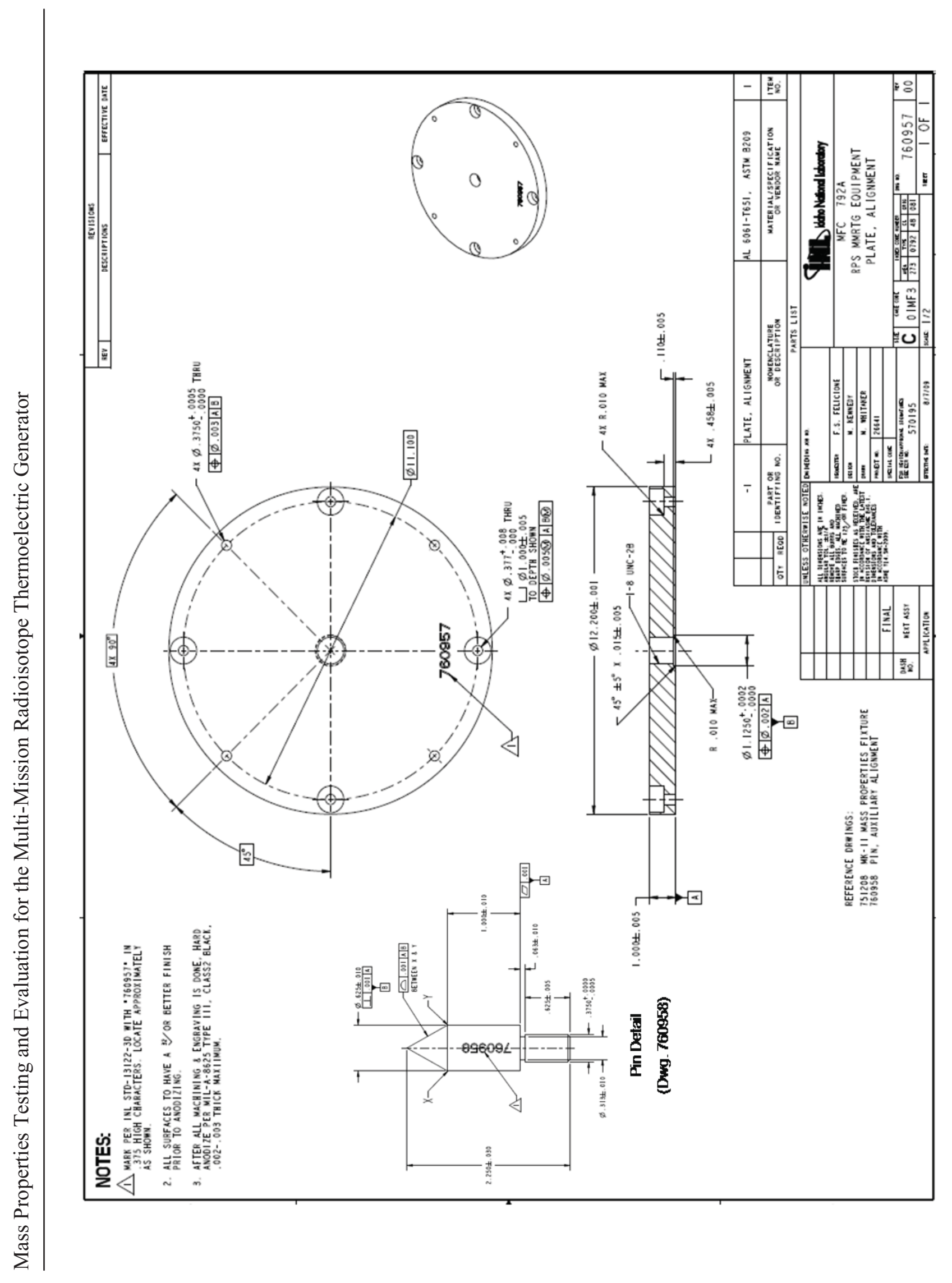

in 
(-) Cradle alignment pin

- Auxiliary flange upper alignment pins

(o) Auxiliary flange lower alignment pin

Primed axes refer to the test article; unprimed axes refer to the MP instrument

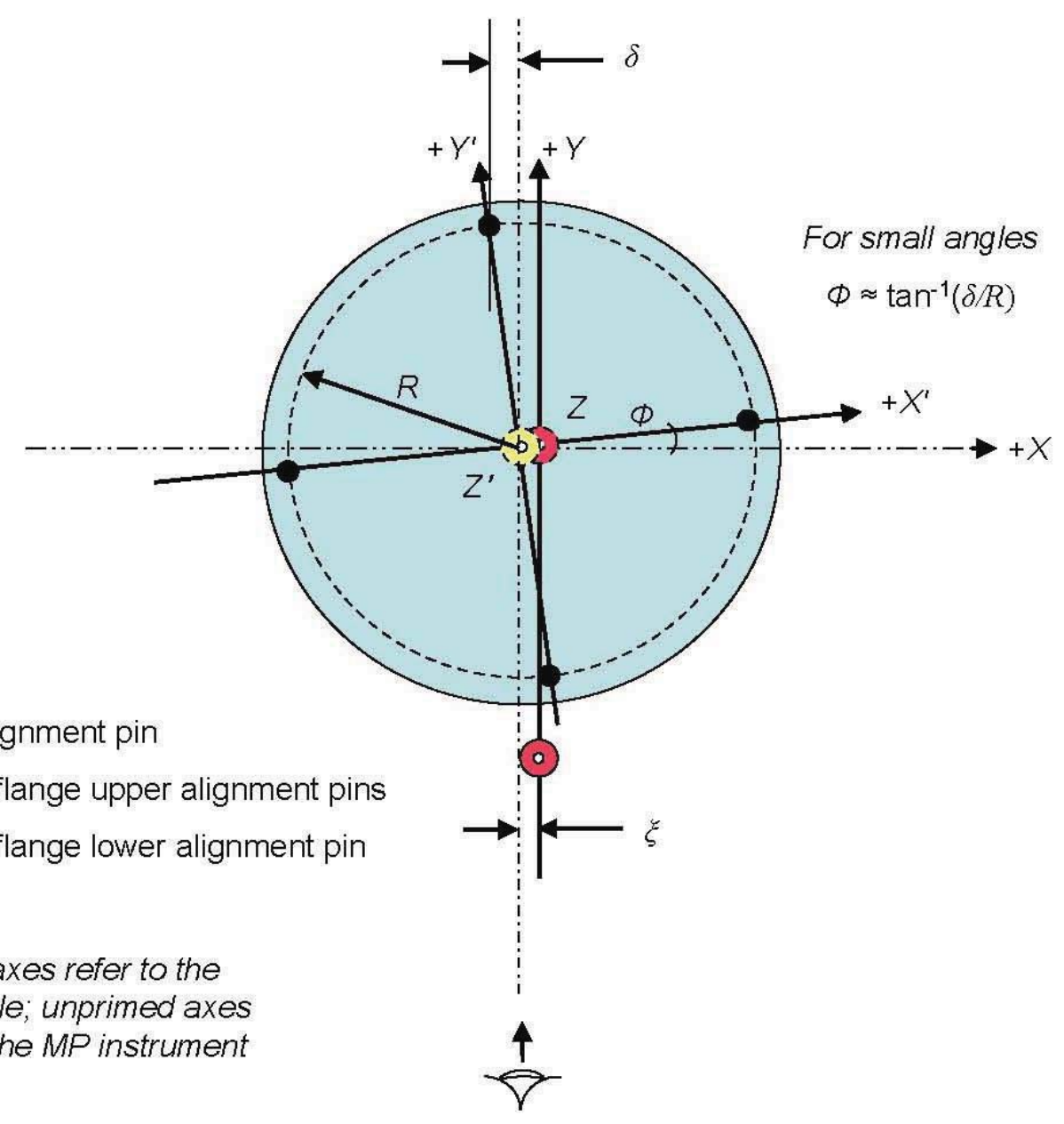

View through transit

Figure 13. Rotational alignment of the test article and MP instrument 


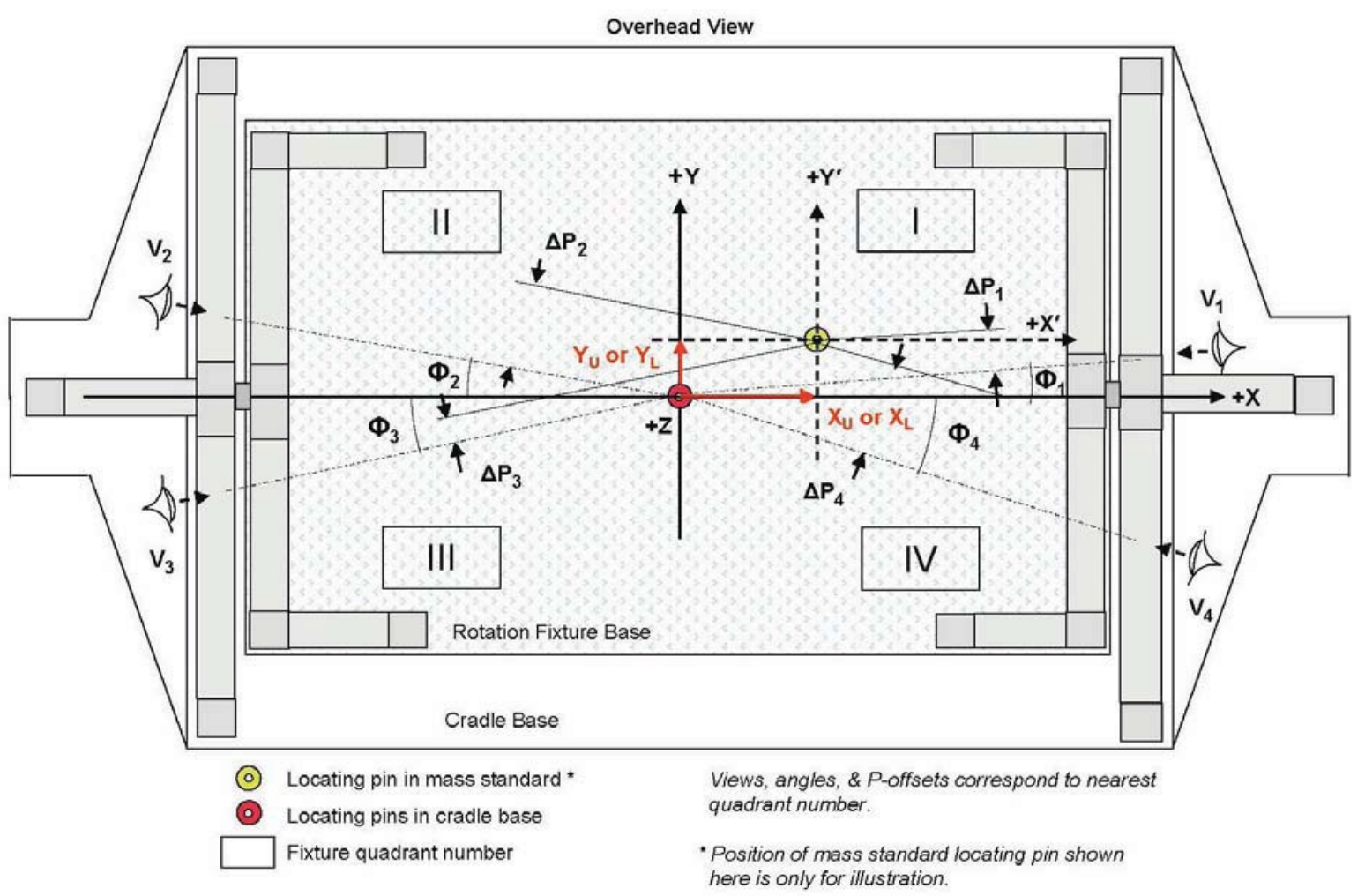

Figure 14. Oblique viewing angles to determine the $\mathrm{Y}$ offset of the test article coordinates 


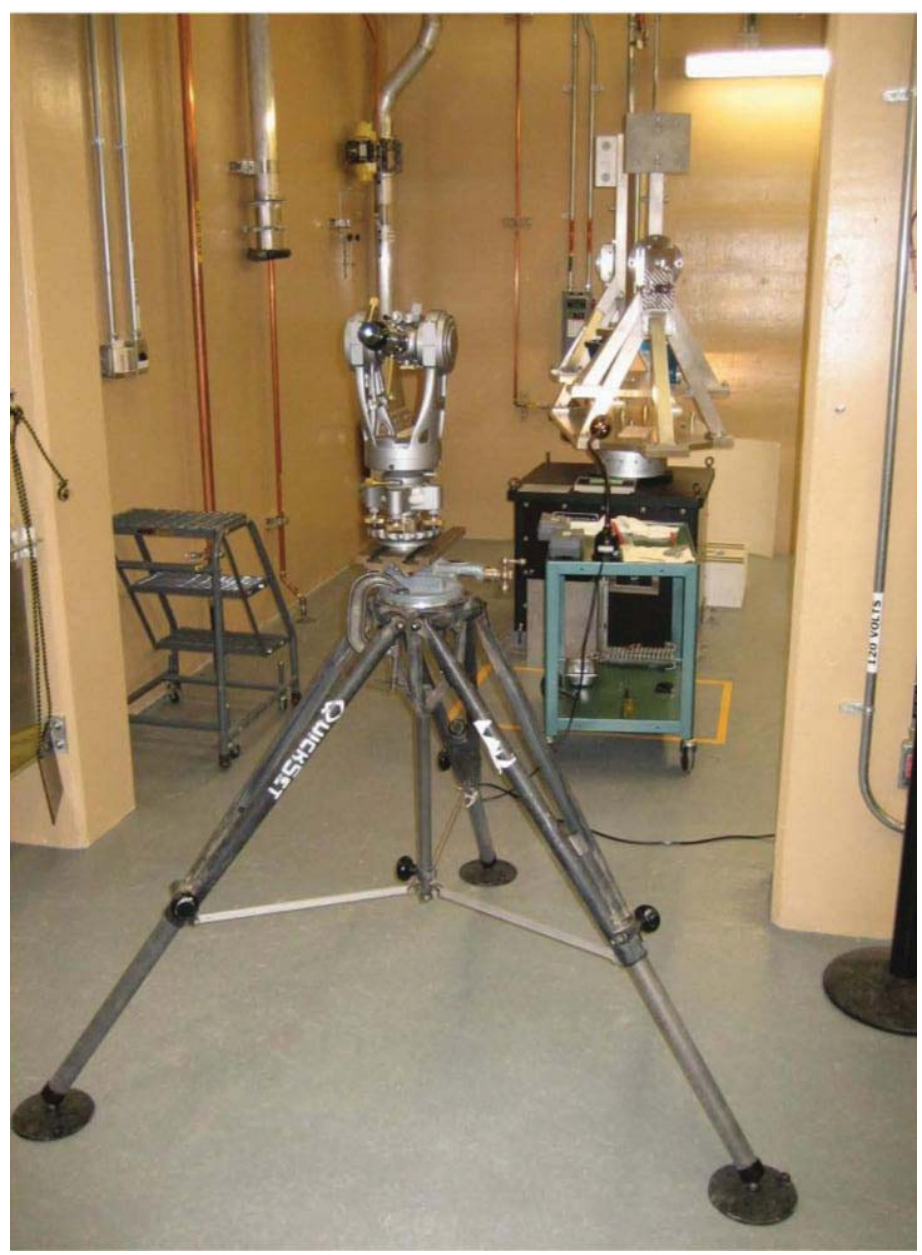

Figure 15. Oblique perspective to determine $\mathrm{Y}$ offset 
Note: + - signs in the view labels and figure caption indicate the applicable signs for the axes. For this construction $A$ \& $\triangle P$ are always positive.
Locating Pins, Position No. 1

Locating pin in cradle base center

Locating pin in mass standard

Alternate position satisfying criteria

Measured: $\Phi, \Delta P$

Known: $\Delta \mathrm{X}$

View No. $2(+)$ or No. $4(-)$

$$
\begin{aligned}
& A=\Delta P / \sin (\Phi) \\
& B=A-|\Delta X| \\
& \Delta P_{0}=|\Delta X| \sin (\Phi) \\
& \text { If } \Delta \mathrm{P}>\Delta \mathrm{P}_{0} \\
& \text { for } V 2(+) i=+1(\Delta Y>0) \\
& \text { for } V 4(-) \quad i=-1(\Delta Y<0) \\
& \text { If } \Delta \mathrm{P}<\Delta \mathrm{P}_{0} \\
& \text { for } V 2(+) i=-1(\Delta Y<0) \\
& \text { for } V 4(-) \quad i=+1(\Delta Y>0) \\
& \Delta \mathrm{Y}=\mathrm{i}|\mathrm{B}|{ }^{\star} \tan (\Phi)
\end{aligned}
$$

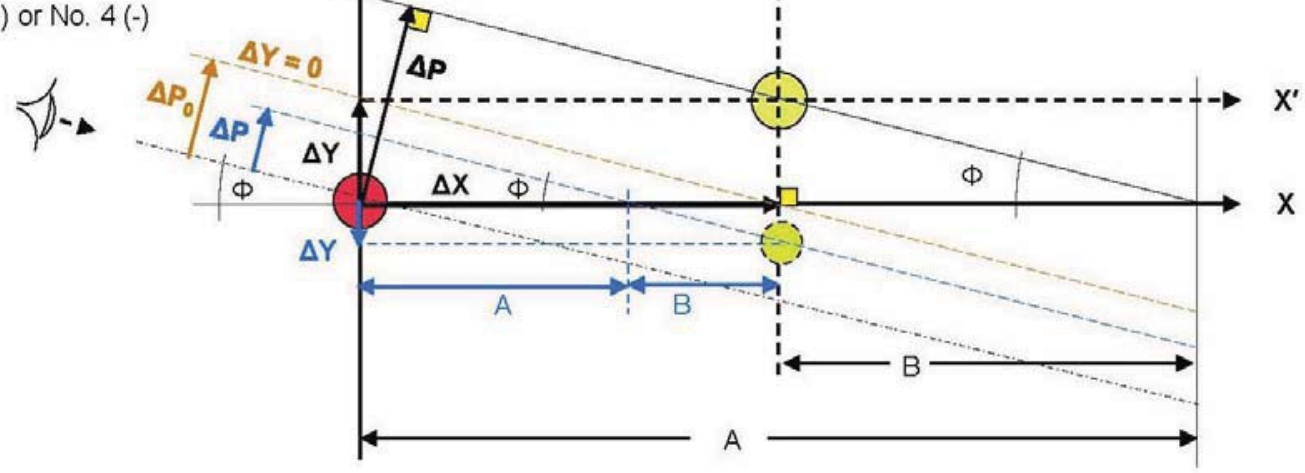

Selected construction depends on view, position of the cradle pin, and direction (left or right) of $\triangle P$.

Figure 16. Typical construction to determine $\Delta Y$ 


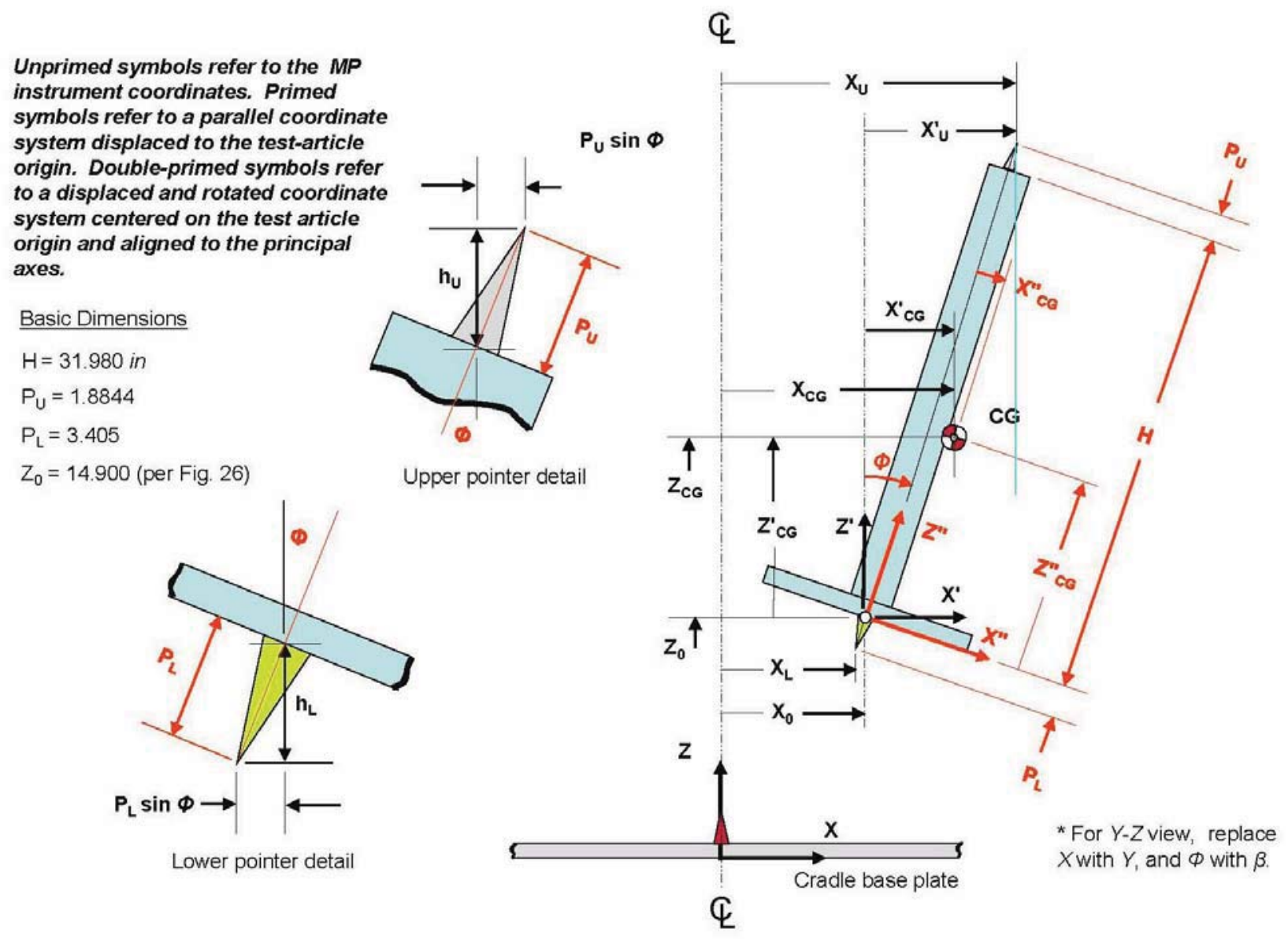

Figure 17. Tilt geometry model in X-Z view (exaggerated) 


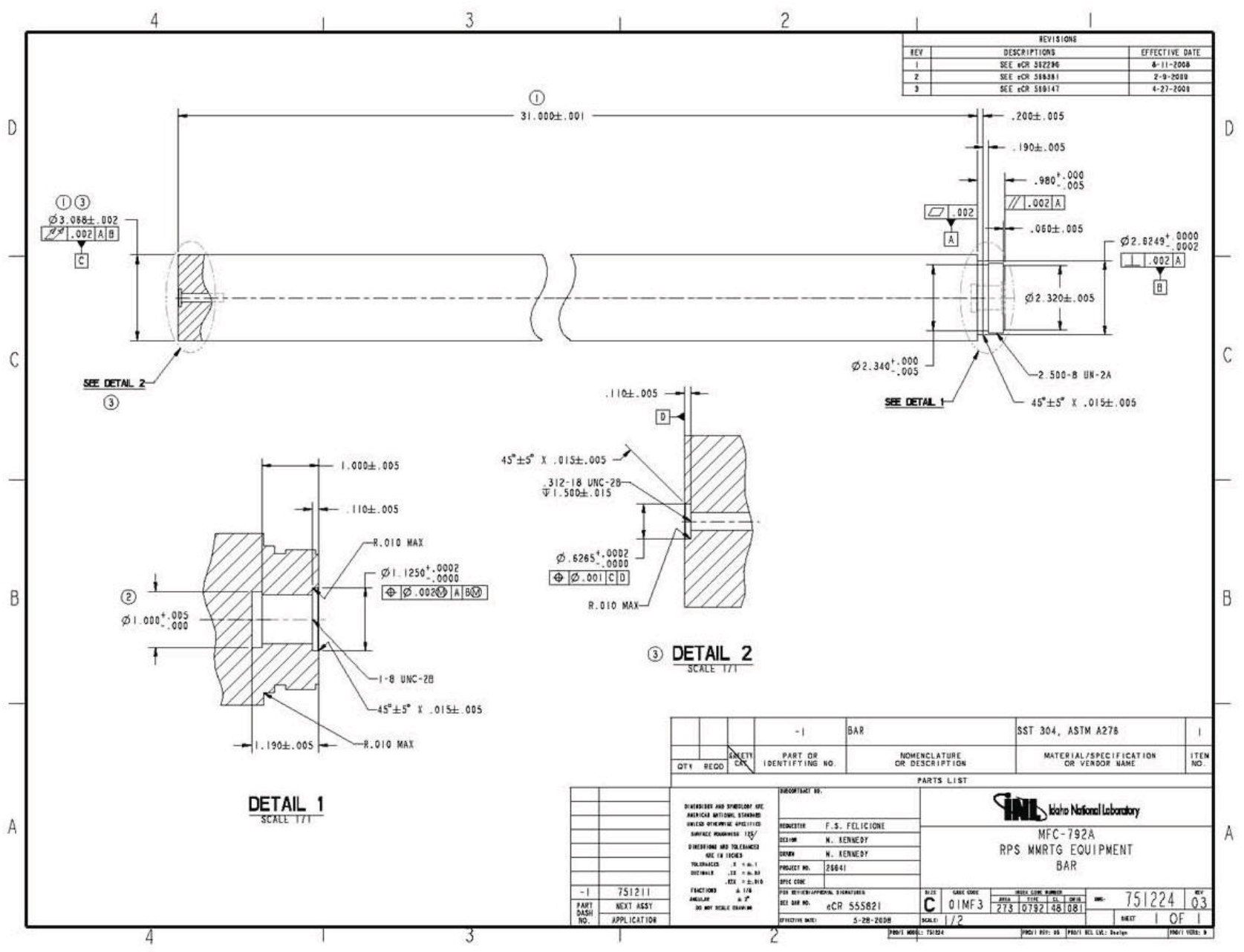

Figure 18. Mass standard column 


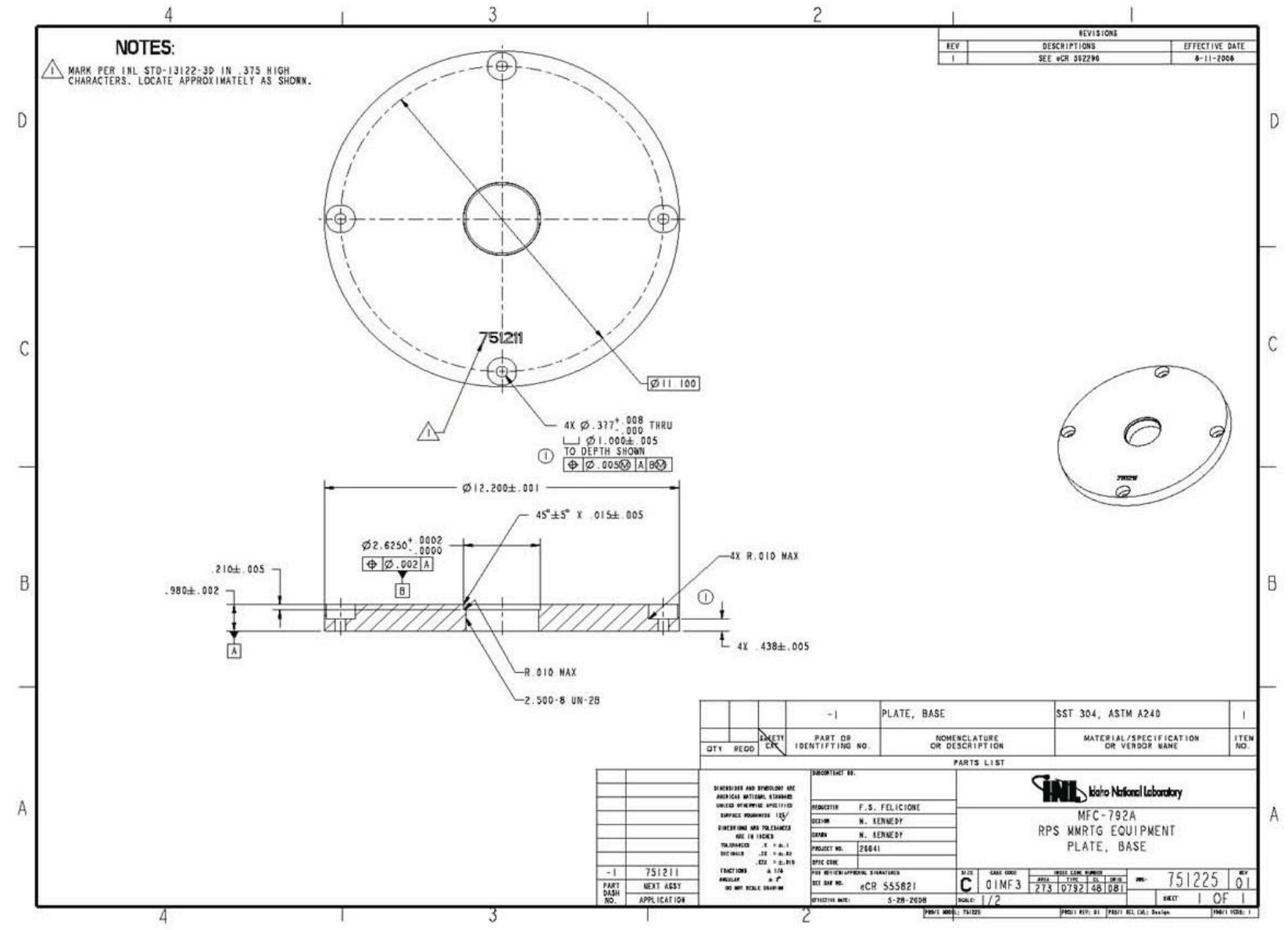

Figure 19. Mass standard base flange 


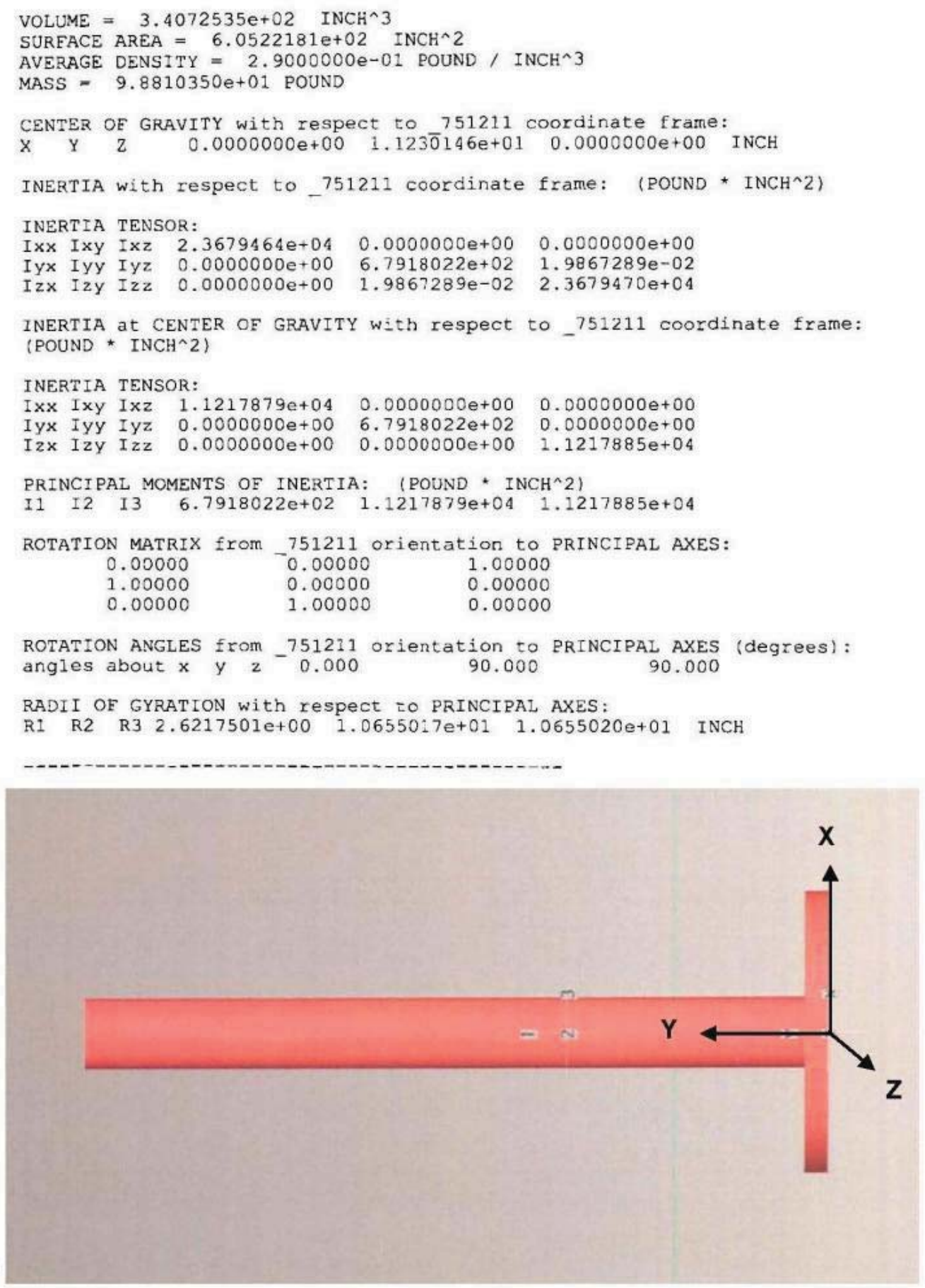

Figure 20. Pro-E software determination of CG in the mass standard 


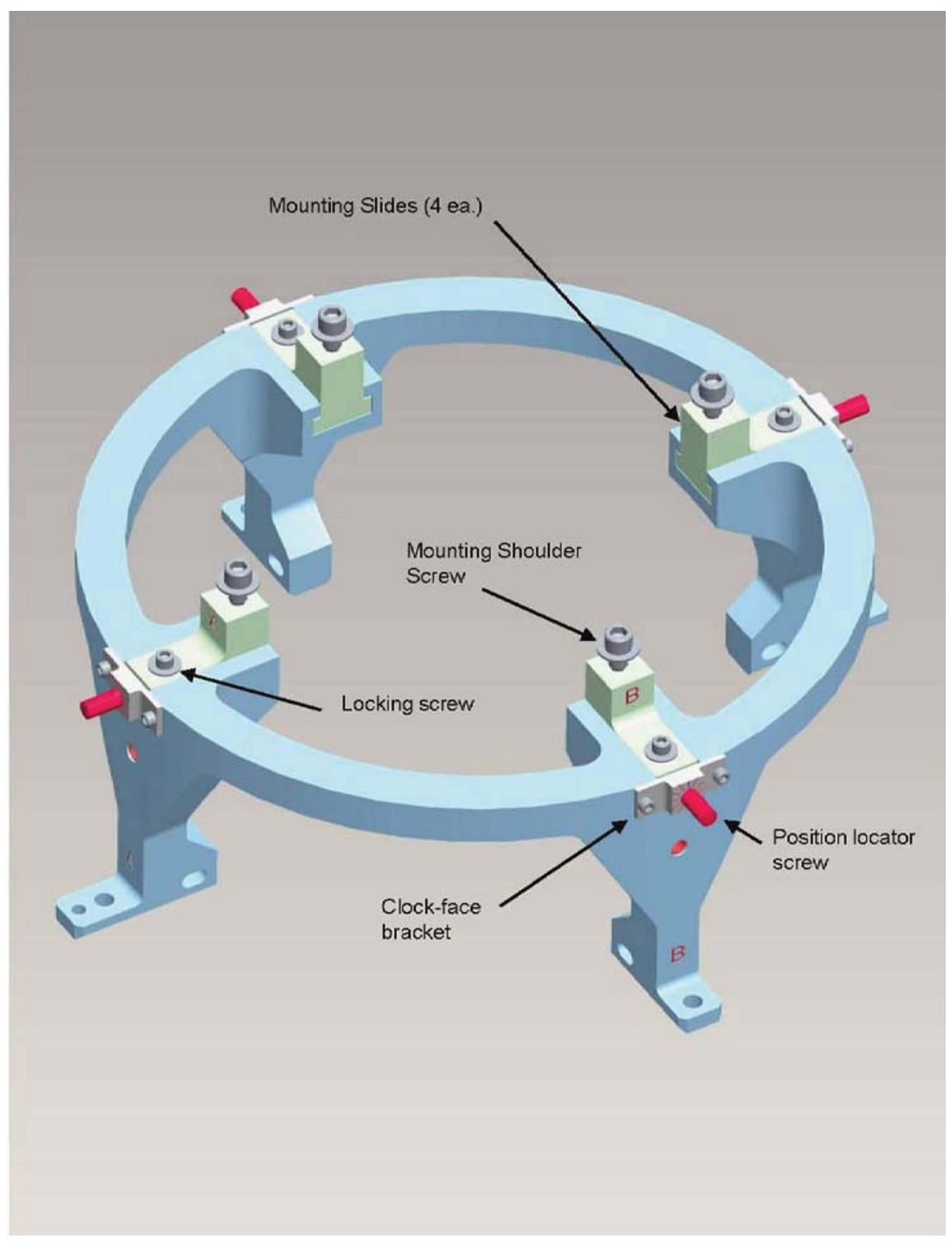

Figure 21. Mk-II (aluminum) adapter ring alignment features 


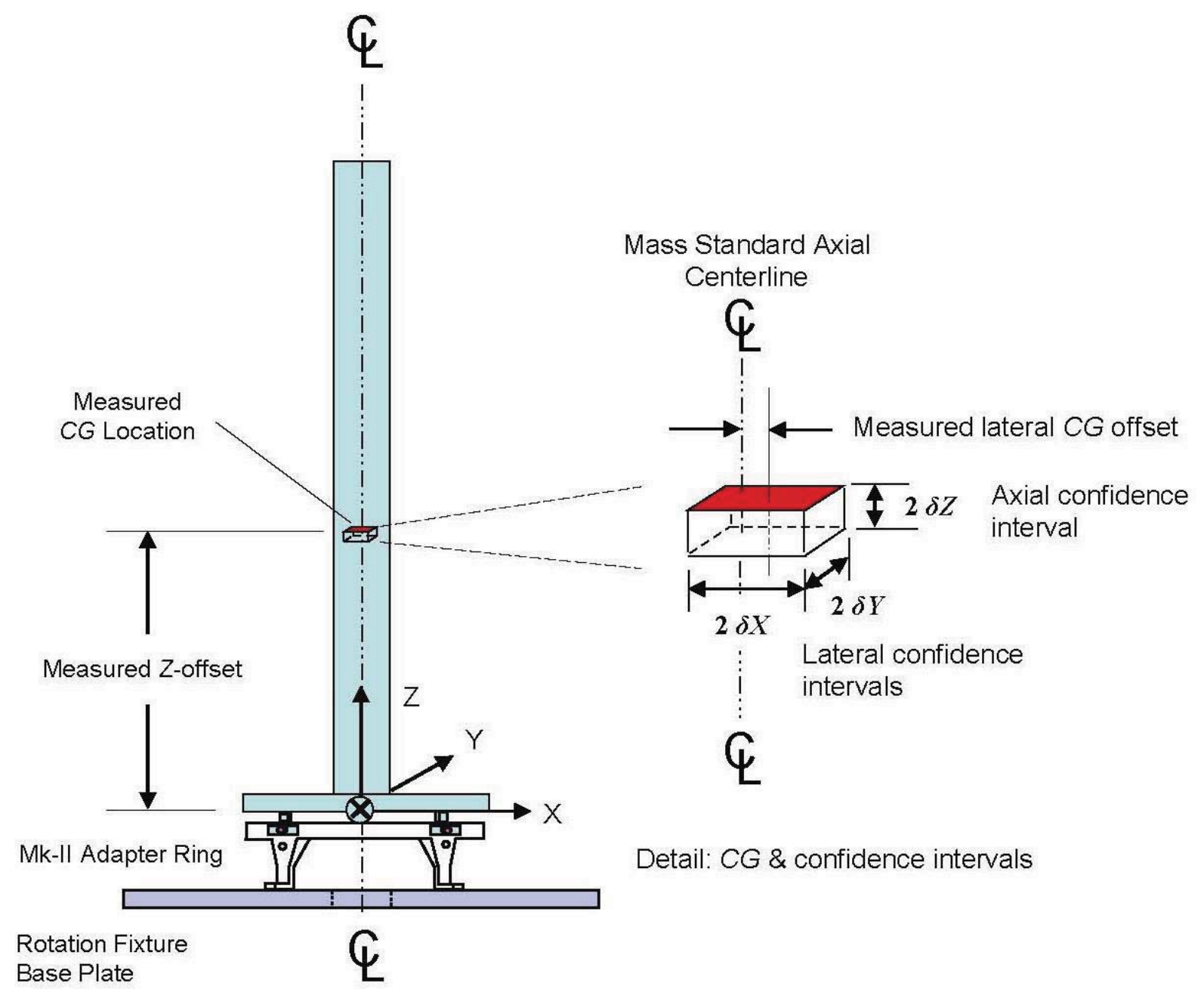

Figure 22. Measured CG location and confidence intervals for the mass standard 


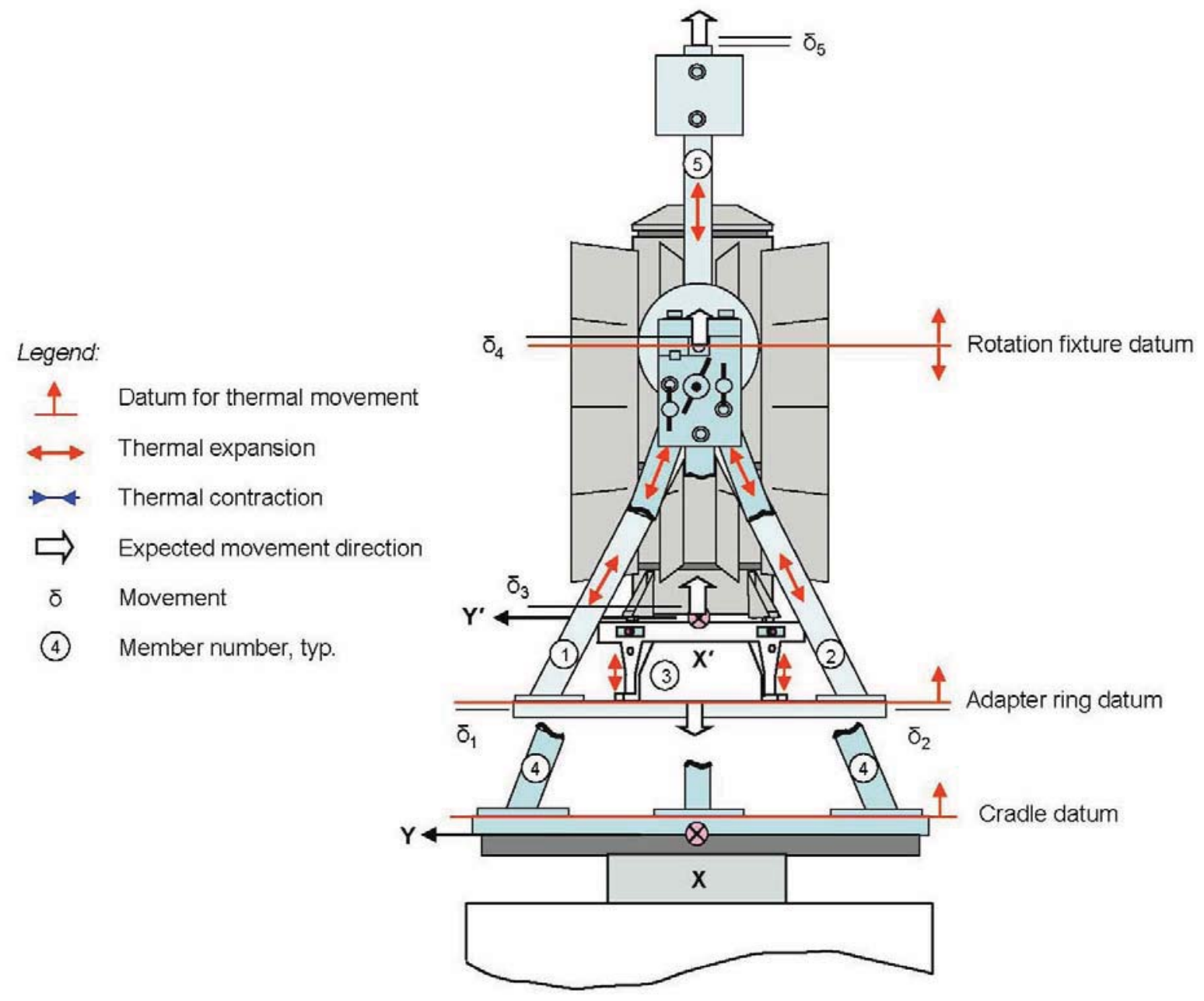

Figure 23. Thermal effects after mounting the MMRTG 


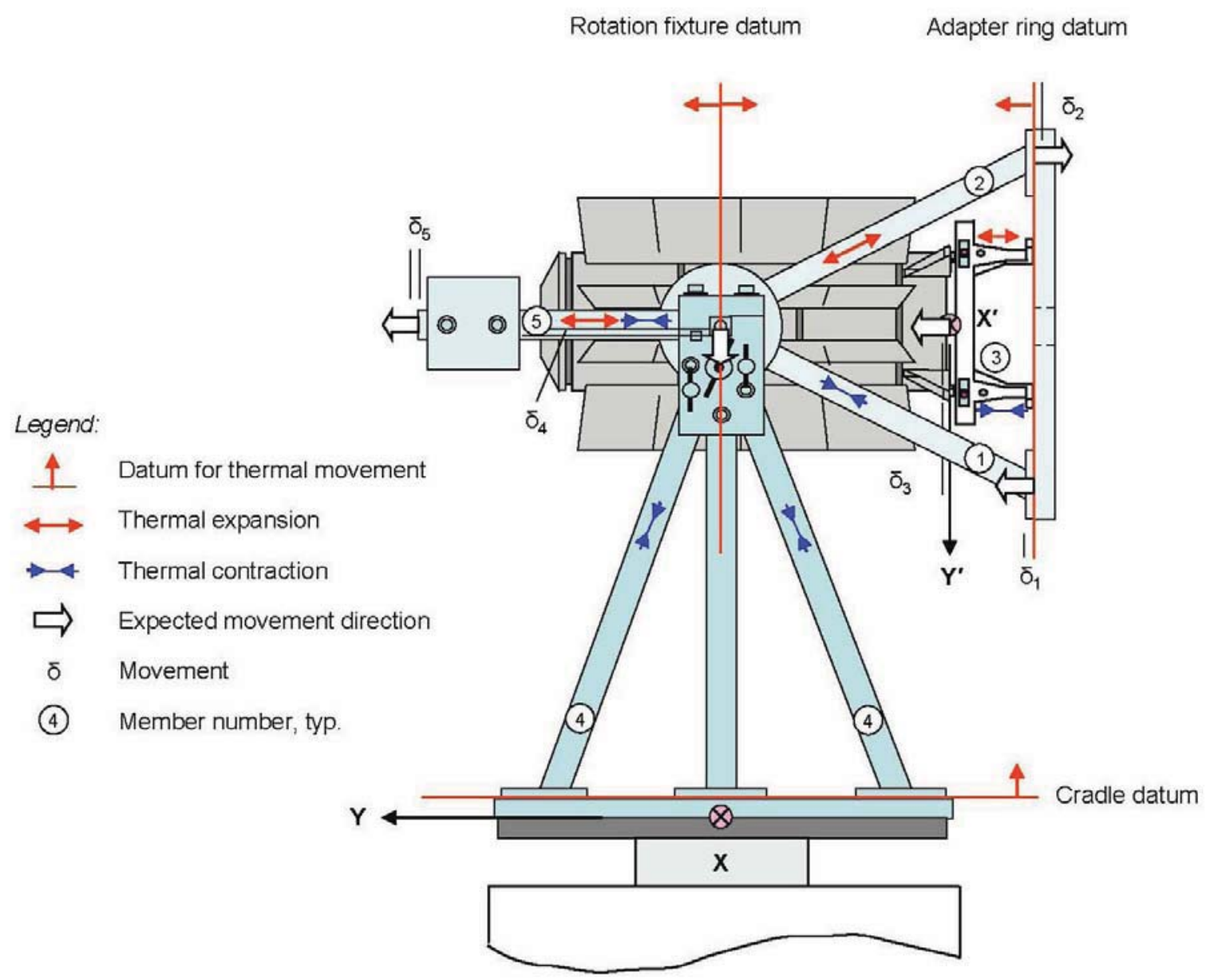

Figure 24. Expansion/contraction changes after rotation to Position No. 2. 


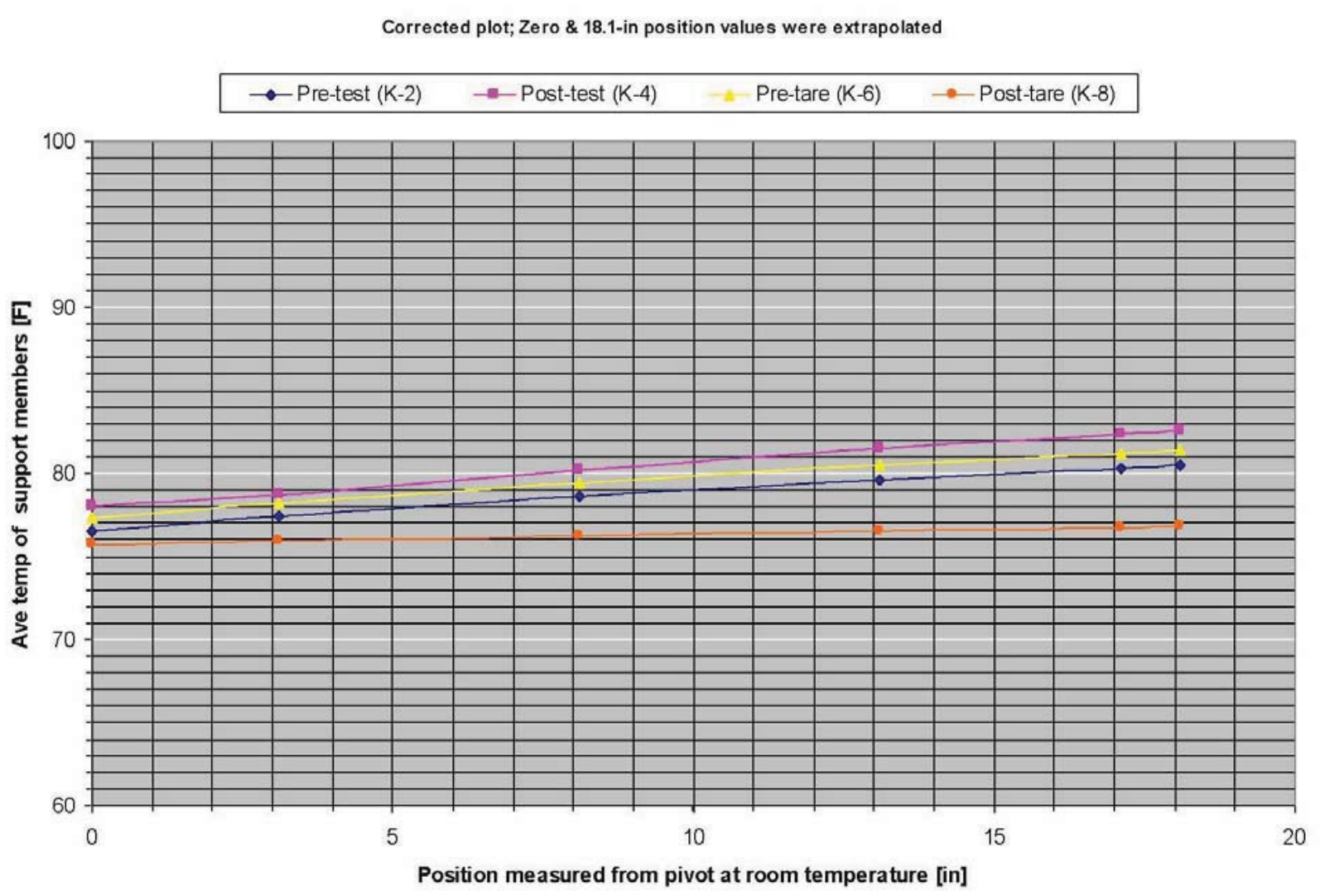

Figure 25. Temperature distributions in the rotation fixture (see temperature maps, Appendix D) 
Basic Geometric Dimensions: $L_{C}=26.000$ in $L_{R}=18.100$ in $L_{A R}=7.004$ in $P_{L}=3.405$ in $Y_{0}$ (Test article datum offset from pivot axis) $=L_{R}-L_{A R}=18.100-7.004=11.096 \mathrm{in}$

Measured Data: $Y_{2}$ (Space Electronics $Y$-component measurement, Position No. 2)

$Z_{C G}^{\prime}=Y_{2}-Y_{O}$ (algebraic)

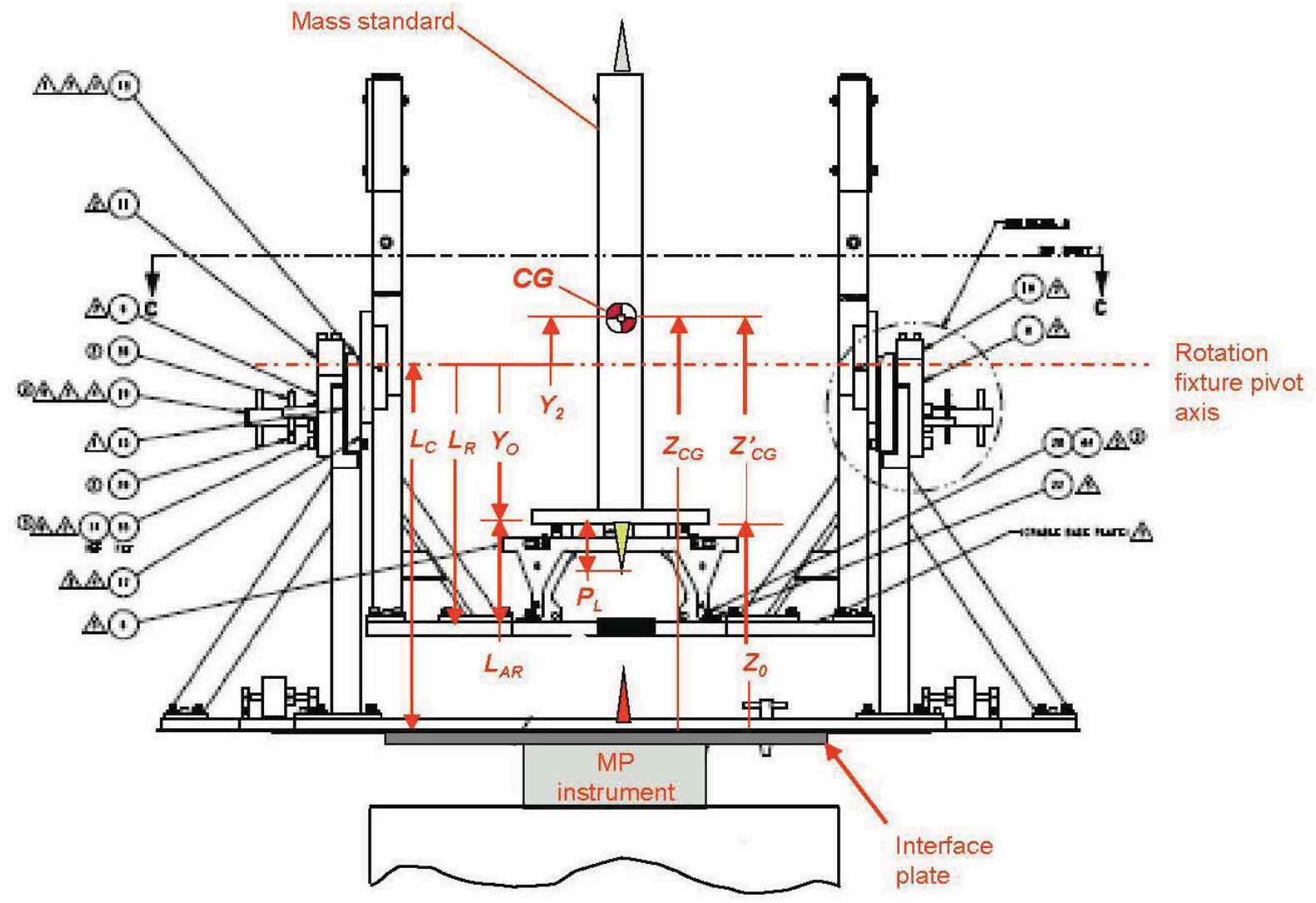

Figure 26. Key dimensions for the mounting fixtures and the test article CG position 

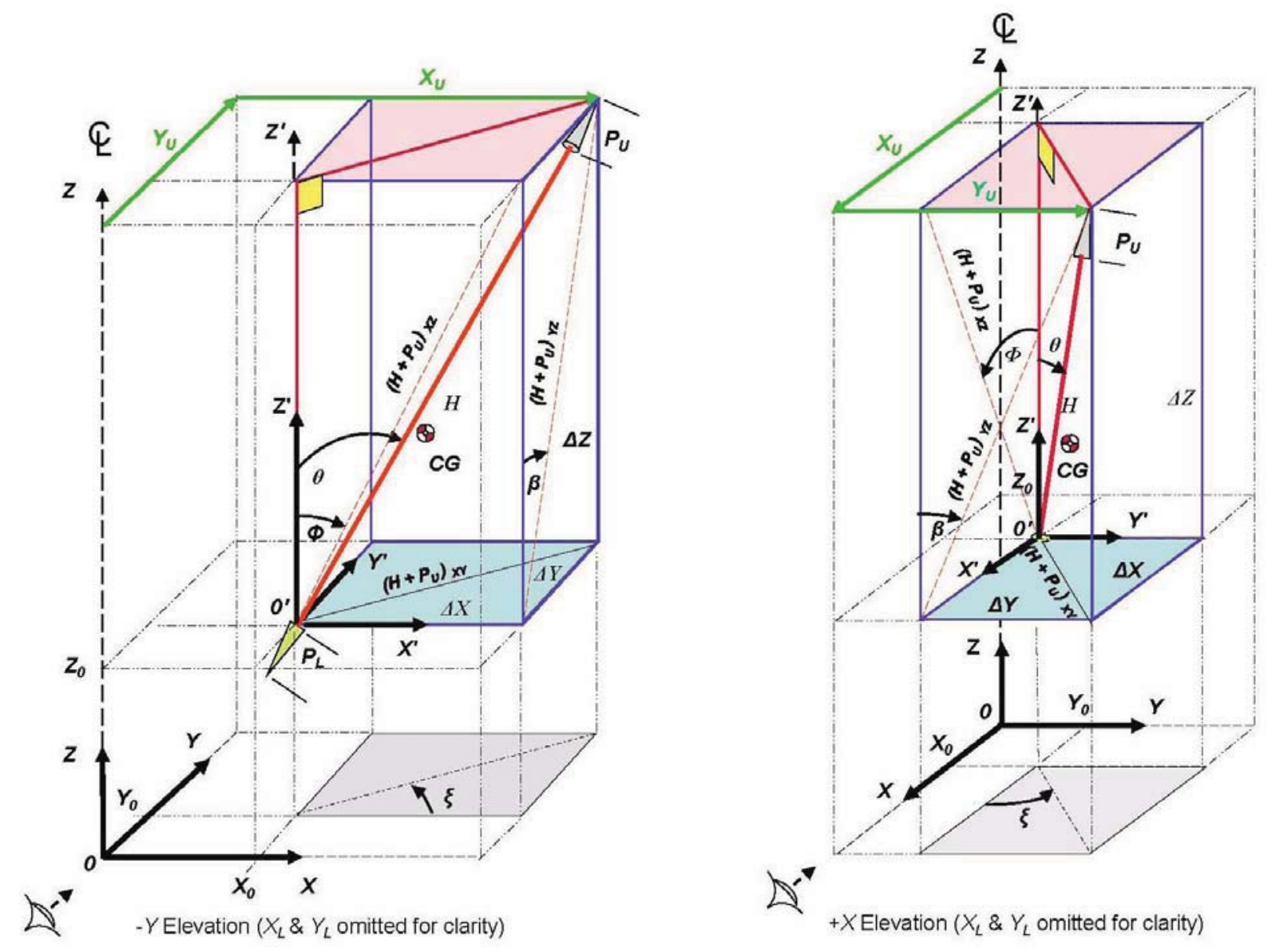

Figure 27. Alignment geometry, isometric views (see Figures 28 \& 29) 


$$
\begin{aligned}
& \text { Lower locator pin } \\
& \text { Upper locator pin } \\
& -1 \text { View direction }
\end{aligned}
$$
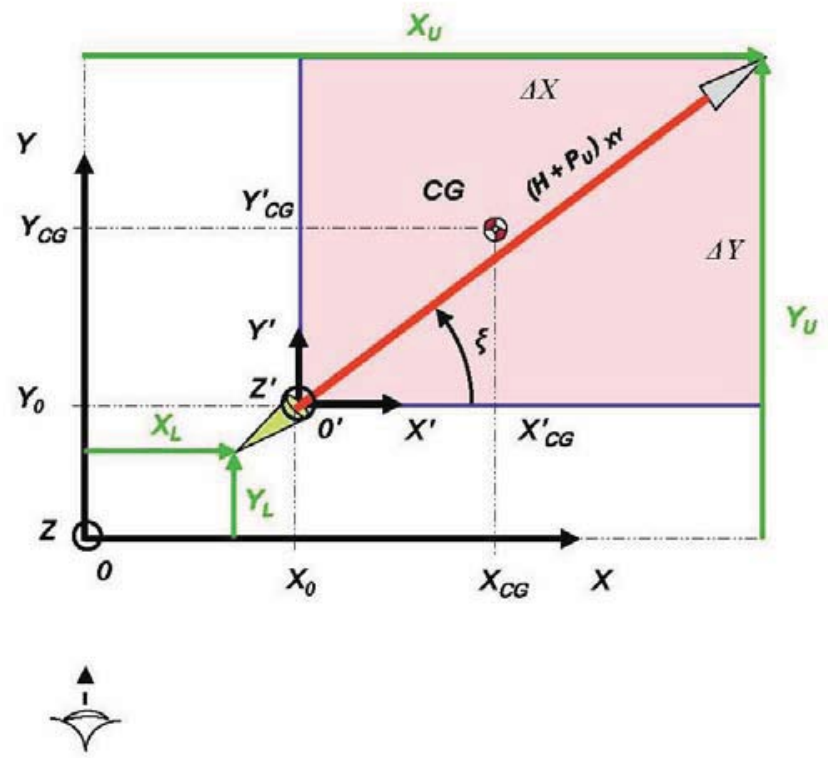
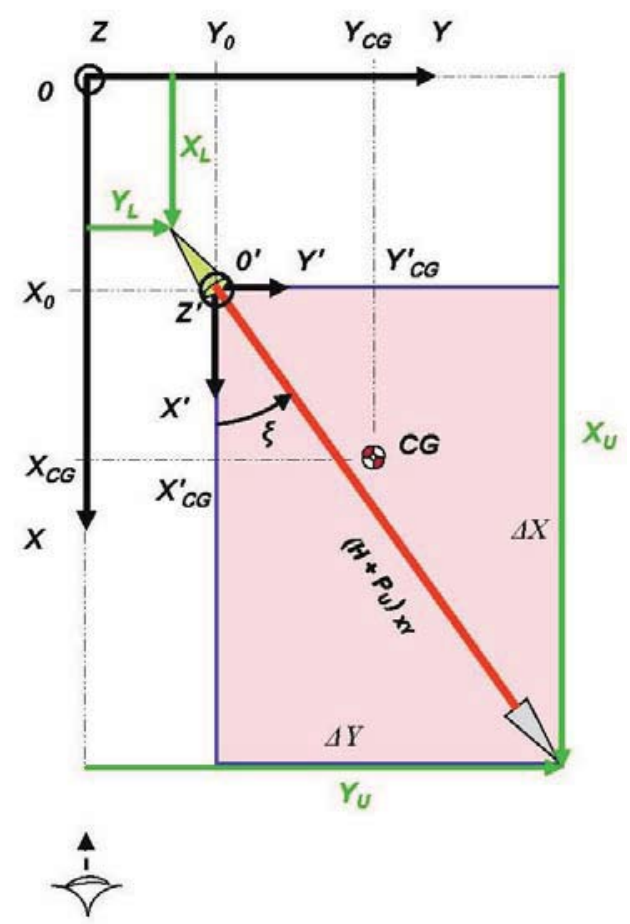

Figure 28. Alignment geometry, overhead views, X-Y, Position No. 1 

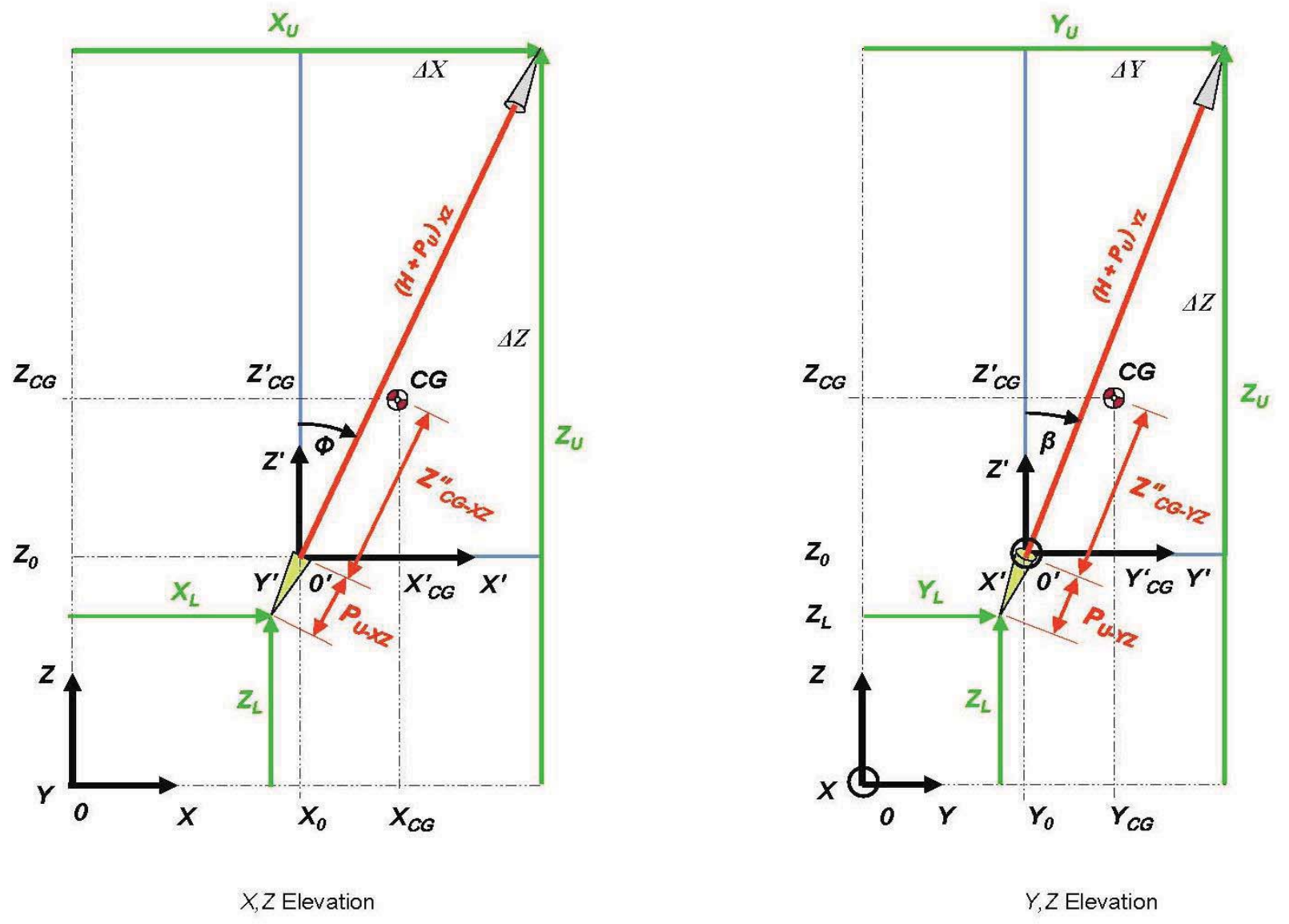

Figure 29. Alignment geometry, elevation views 


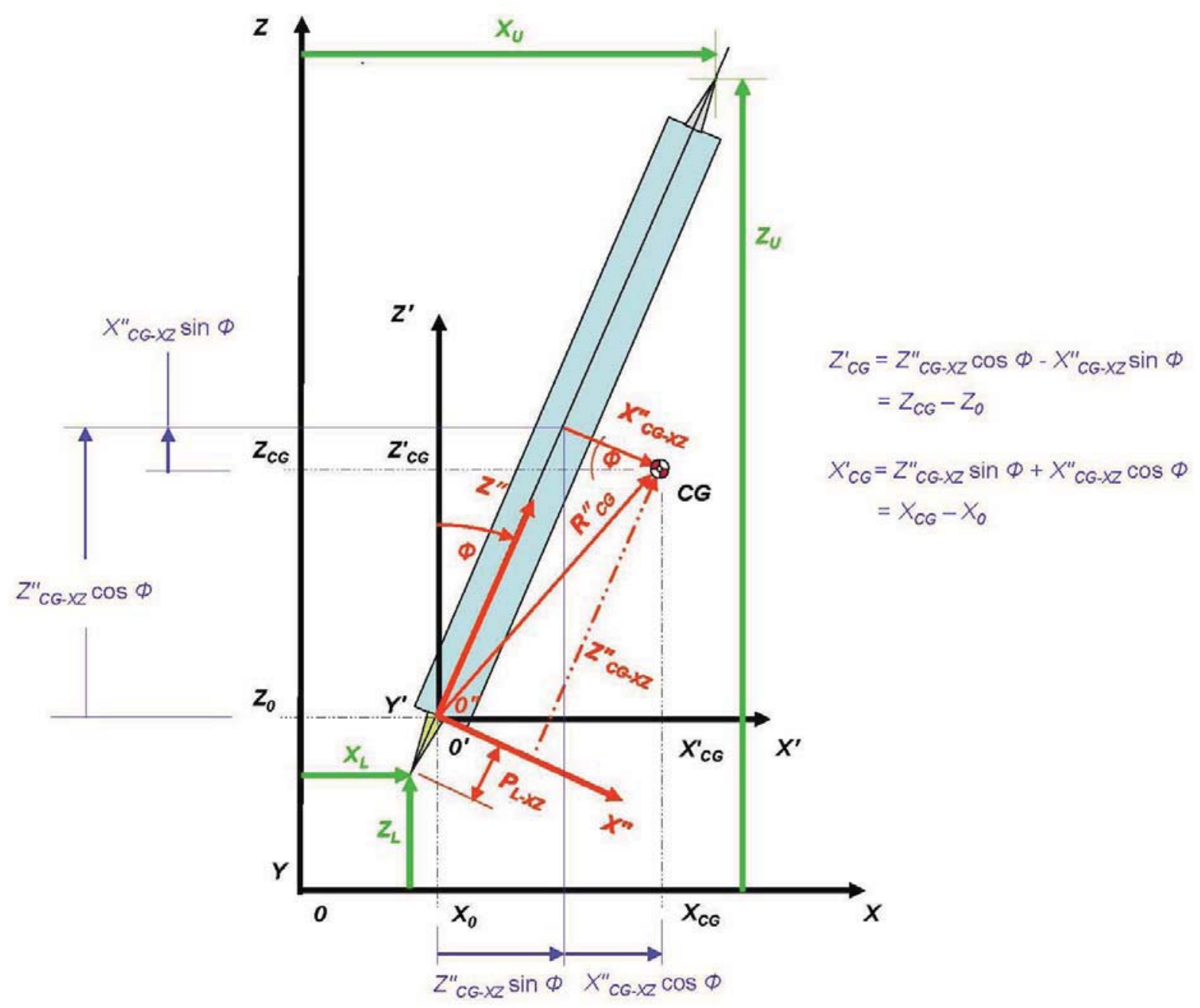

Figure 30. Tilt Analysis: X-Z Elevation View 


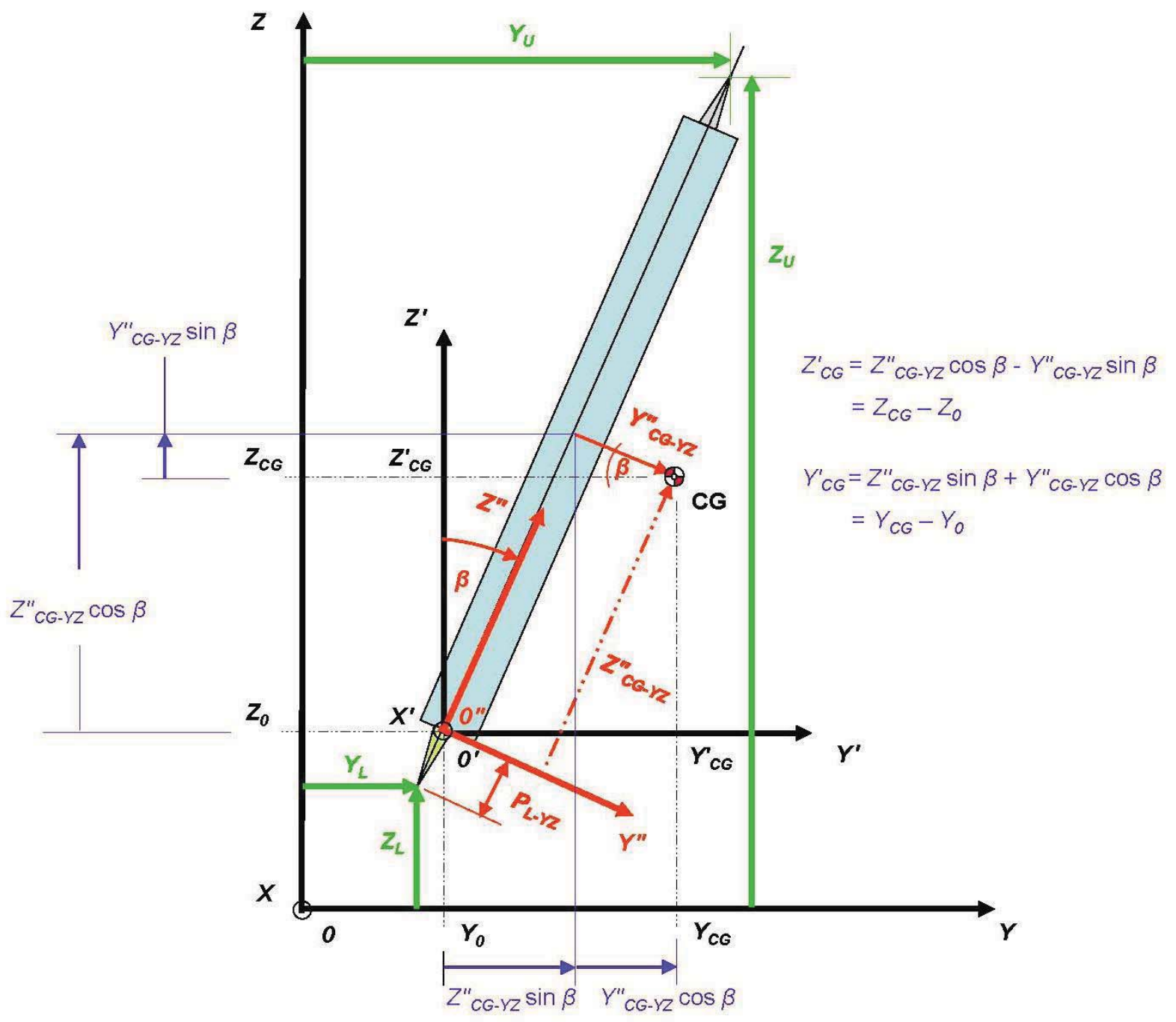

Figure 31. Tilt analysis: Y-Z Elevation View 
INTENTIONALLY BLANK 


\section{Appendix A}

\section{INL Mass Properties Testing System Drawings List}




\section{Appendix A \\ INL Mass Properties testing System Drawings Listing}

\section{Drawing}

Number Titlen

751208 Mk-II Mass Properties Fixture Assembly

751209

751210

751211

751212

751213

751214

751215

751216

751217

751218

751219

751220

751221

751222

751223

751224

751225

751226

751227

751228

751229

751230

751231

751232

751233

751234

751235

751236

751237

751238

751239
Mk-II Rotation Fixture Assembly

Mk-II Adapter Ring Assembly

Mk-II Dummy Qualification Mass Assembly

Mk-II Mass Properties/Rotation Fixture Lifting Assembly

Mk-II Pivotable Balance Assembly

Base, Adapter Ring

Slide A, Mounting

Bracket, Locating Screw

Screw, Locating

Rotation Fixture Cradle Assembly

Axle, Positioning Stub

Counterbalance

Plate, Counterbalance Mounting

Rod, Pivot

Weight, Balance

Bar

Plate, Base

Pin, Cross Hair Locating

Pin, Locating

Brace, Lifting

T-Bar, Mass Properties Fixture

T-Bar, Rotation Fixture

Plate, Cradle Base

Brace, Cradle Support

Retainer

Cap, Retainer

Yoke

Bracket, Mounting Counterbalance, Inside

Bracket, Mounting Counterbalance, Outside

Plate, Yoke Spacer

Plate, Sliding Shim

n All drawings include the building number, MFC-792A, and the topic designation, RPS MMRTG [Equipment] in their titles. These are omitted in the list in this appendix. 
$751240 \quad$ Pin, Rotation Fixture Positioning

$751241 \quad$ Pin, Cradle Locating

$751242 \quad$ Pin, Alignment

$751243 \quad$ Key, Retainer

$751244 \quad$ Counterbalance

$751245 \quad$ Rod, Counterbalance Mounting

751246 Pin, Adapter Ring Positioning

$753280 \quad$ Mk-II Mass Properties Cradle Assembly

$755602 \quad$ Screw, Special Mounting

$755820 \quad$ Plate, Cradle Base

755821

756288

758211

Brace, Cradle Support

Pin, RTG Set-down Alignment

Spacer, MMRTG Mounting Screw

$759601 \quad$ Close Coupled Y-Cable Socket

759869

Counterweight, Brass

759870

Pin, Alignment (Adaptor Ring to MP Fixture)

760033

Pin, Auxiliary Mass-Simulator Alignment

760120

Push Tool, Sliding Shim

$760957 \quad$ Plate, Alignment

760958 Pin, Auxiliary Alignment 
INTENTIONALLY BLANK 


\section{Appendix B}

\section{Geometric Constructions for Determining Offsets of the Coordinate Systems}




\section{Appendix B \\ Geometric Constructions for Determining Offsets of the Coordinate Systems}

Note: target items that are not lined up with the reference point will lie either to the left or the right of the reference point when viewed in the transit. The micrometer on the transit has a red scale to measure the offset of targets lying to the left of the reference and a black scale to measure targets lying to the right. To ensure that the field metrology data were recorded with the minimum of operator interpretation, the target positions were recorded using the red or black indications accordingly. Constructions in this appendix continued this use of the terminology, Red or Black.

Half of the constructions shown in this appendix, viz., Constructions A, C, E, and G involve viewing angles that would allow the $\mathrm{Y}$ offset of the target pin to be either on the positive or negative side of the $\mathrm{Y}$ axis even though remaining on the same side of the reference pin when viewed in the transit. An "alternate position" target pin symbol is included on these constructions to handle this possibility. The critical displacement for reversal of the axis side is denoted as $\Delta \mathrm{P}_{0}$ on these constructions $\left(\right.$ at $\Delta \mathrm{P}=\Delta \mathrm{P}_{0}$ the $\mathrm{Y}$ offset is zero). The algorithms listed on the constructions properly account for this apparent ambiguity. The circumstances when this can or cannot occur are summarized in Spreadsheet (B-1) (Table 9). ${ }^{\circ}$ This occurs when larger or smaller offsets $\Delta \mathrm{P}$ have the geometric possibility to cause the mass standard target pin to cross the $\mathrm{X}$ axis as the magnitude of $\Delta \mathrm{P}$ varies but without reversing its position relative to the reference pin.

o Spreadsheet(s) from Appendix B have been assigned a sequential table number and are collected with other tables in this report. 


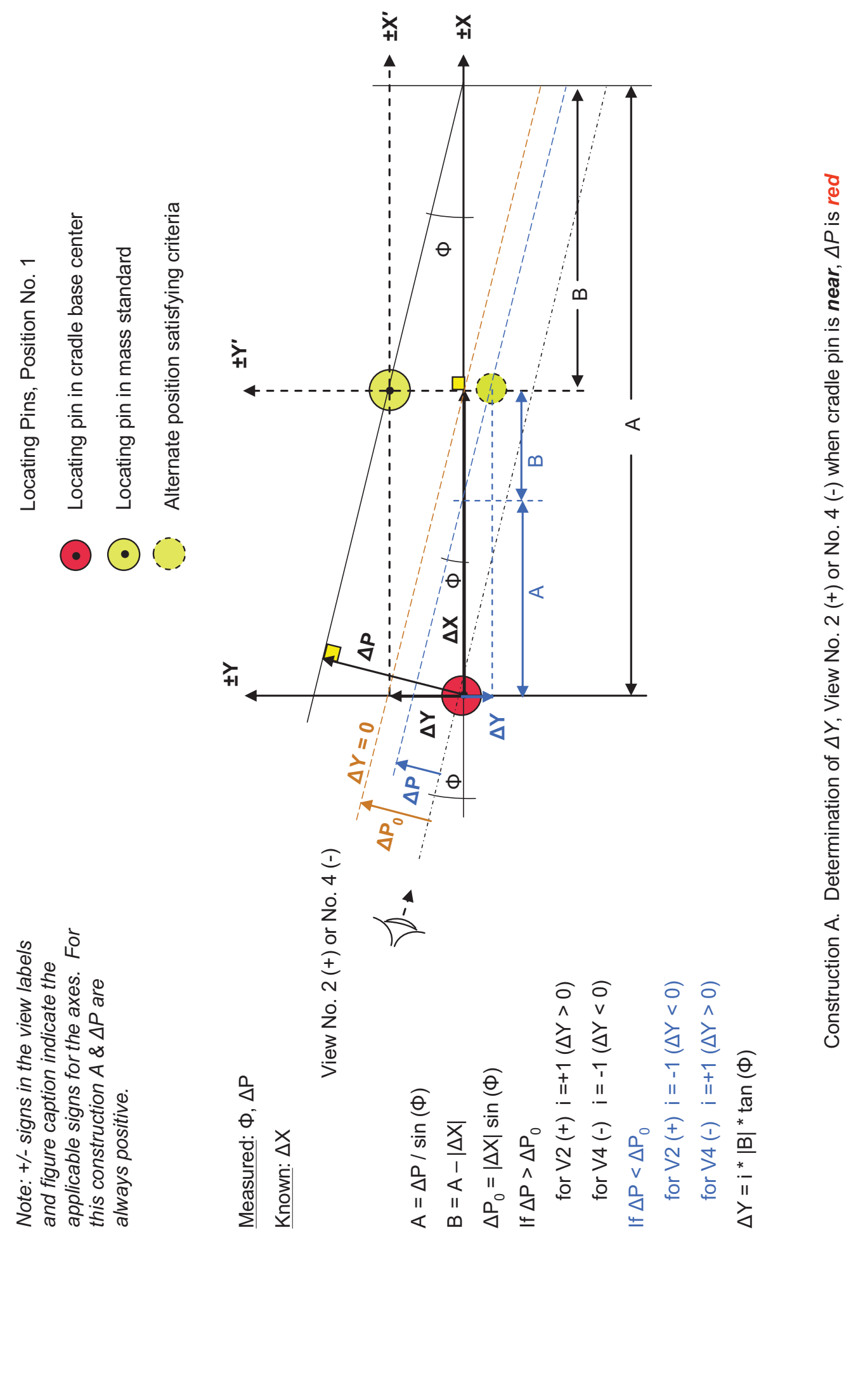



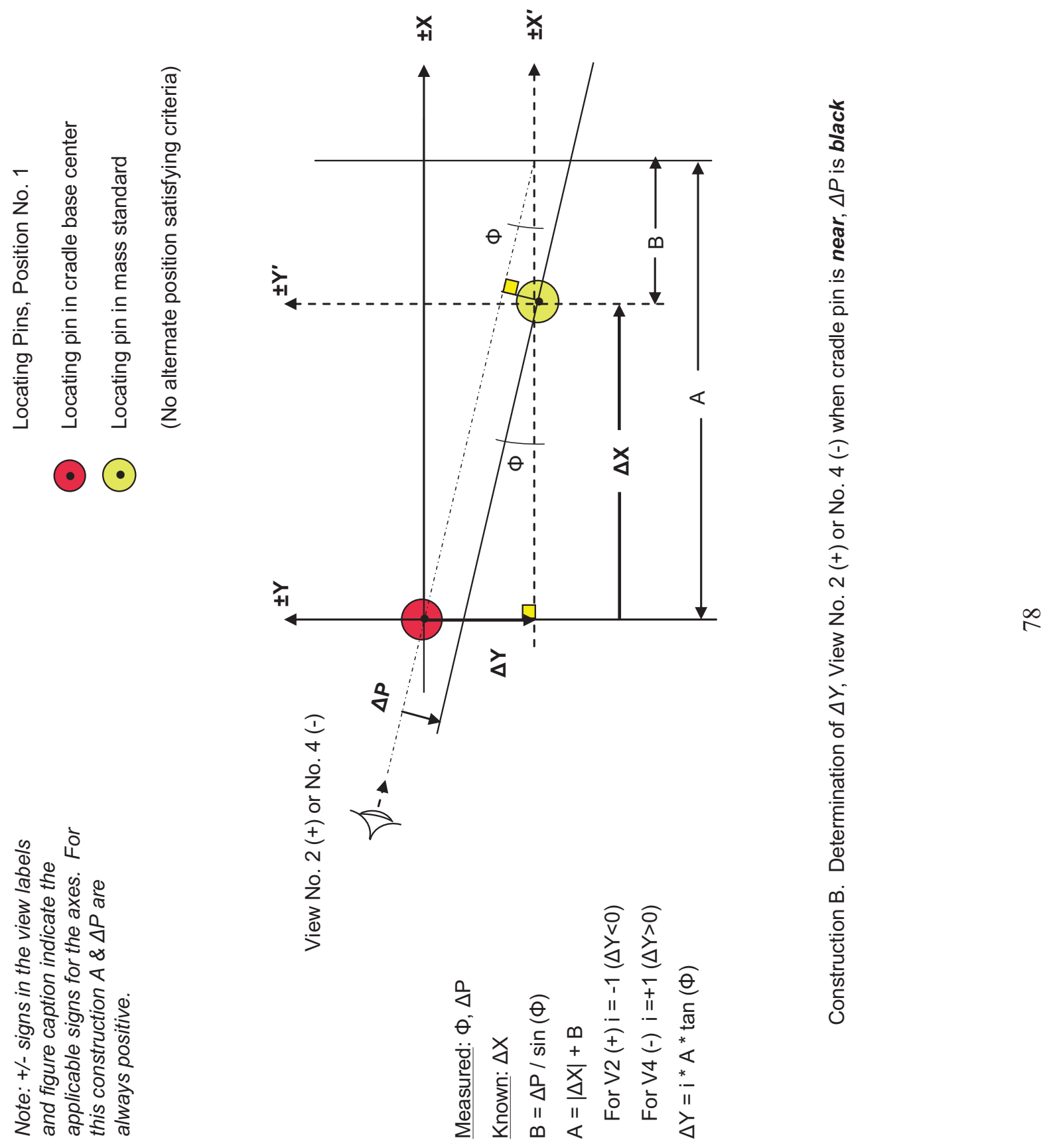


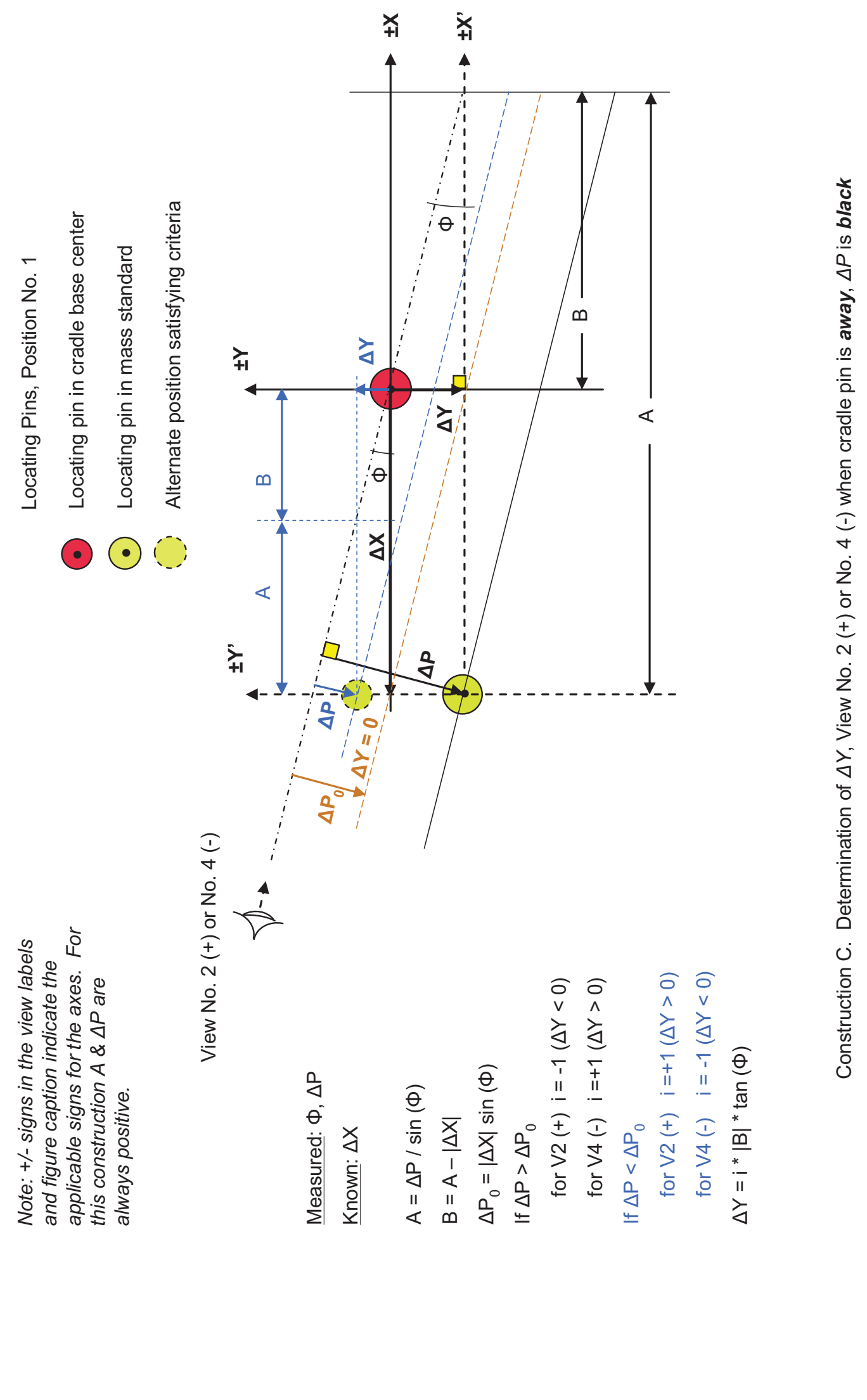



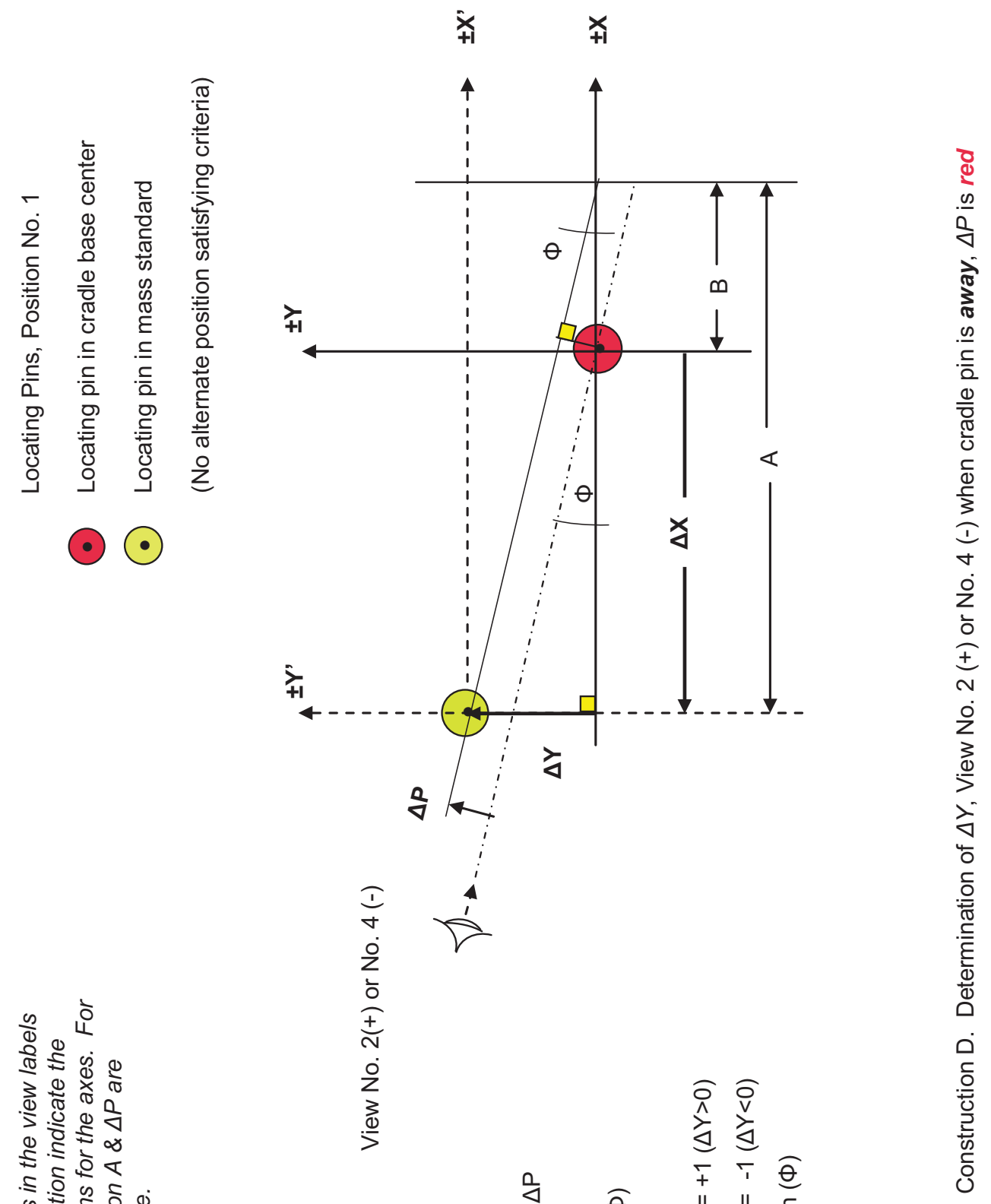

$\leq . \div$

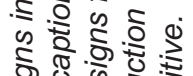

के 0 辛

+ 궁

बi

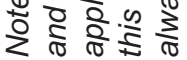

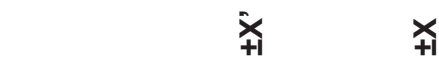

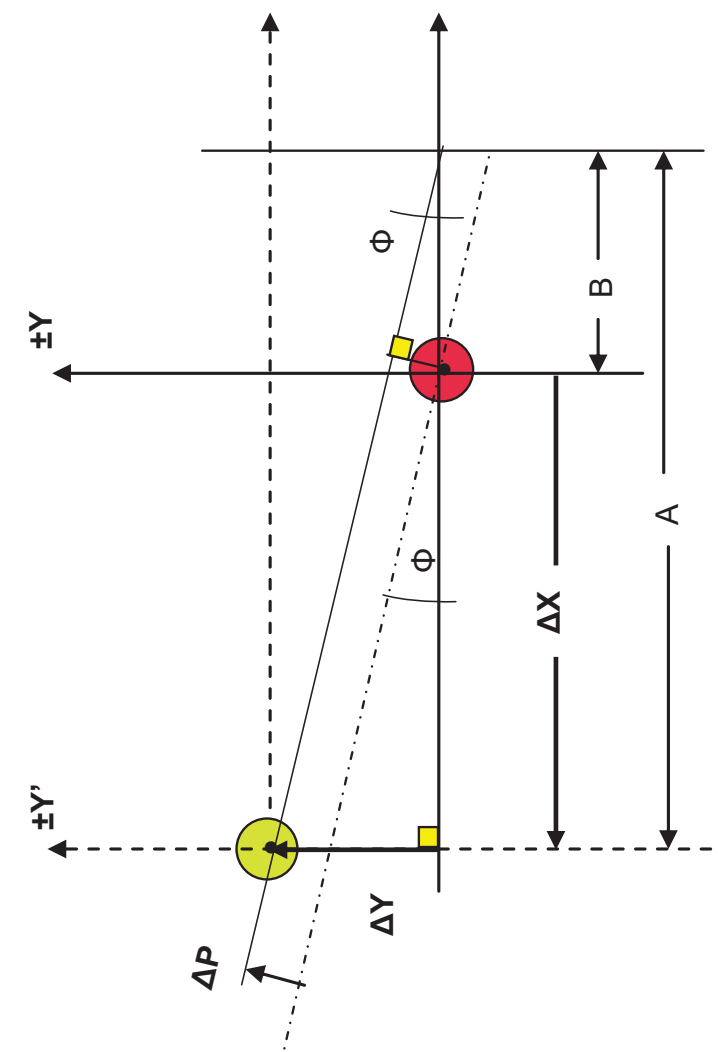

$I$
I
$\dot{2}$

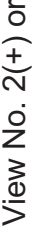



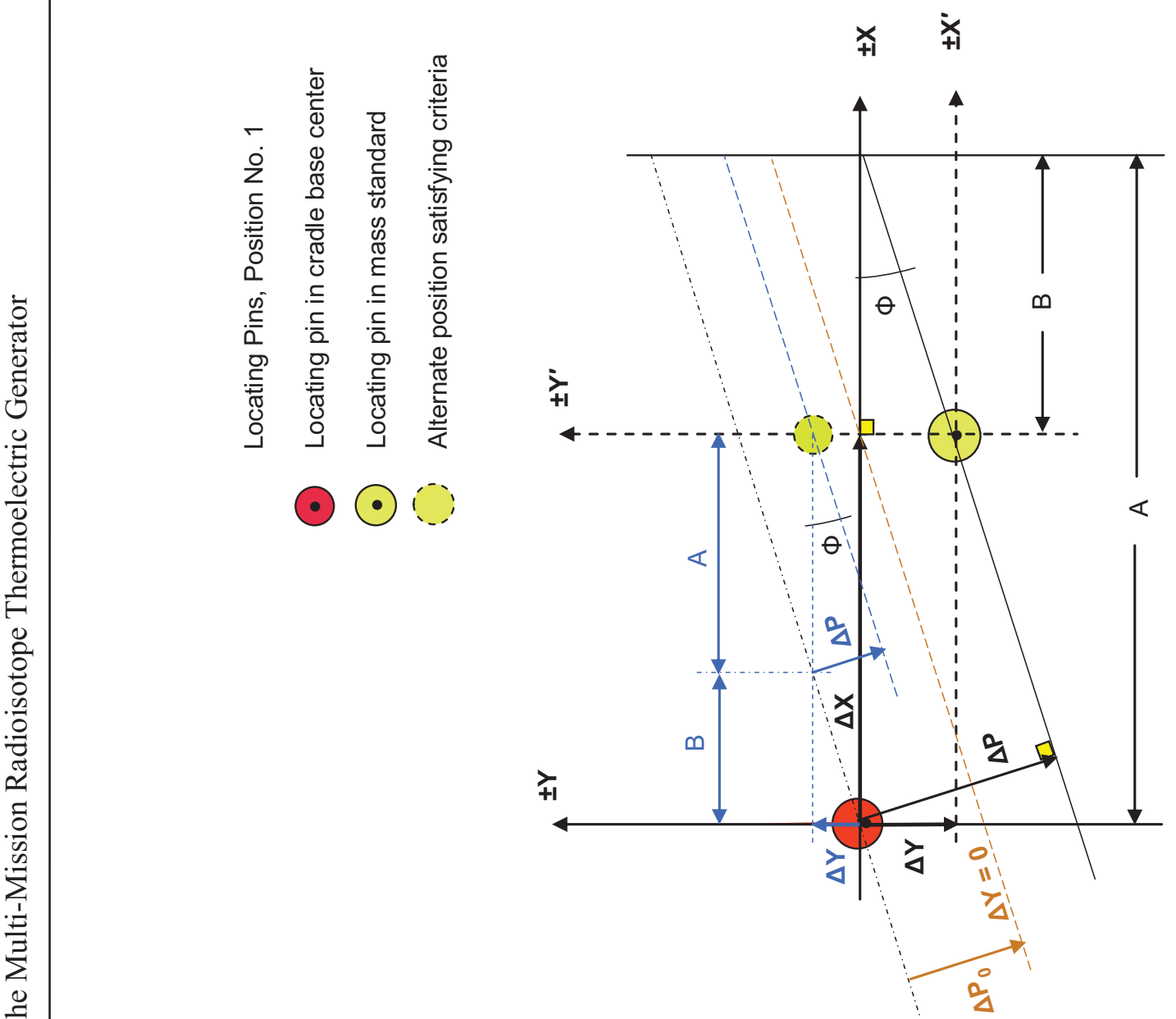

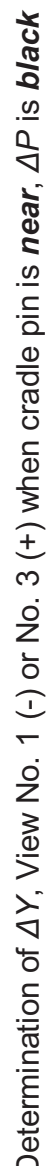

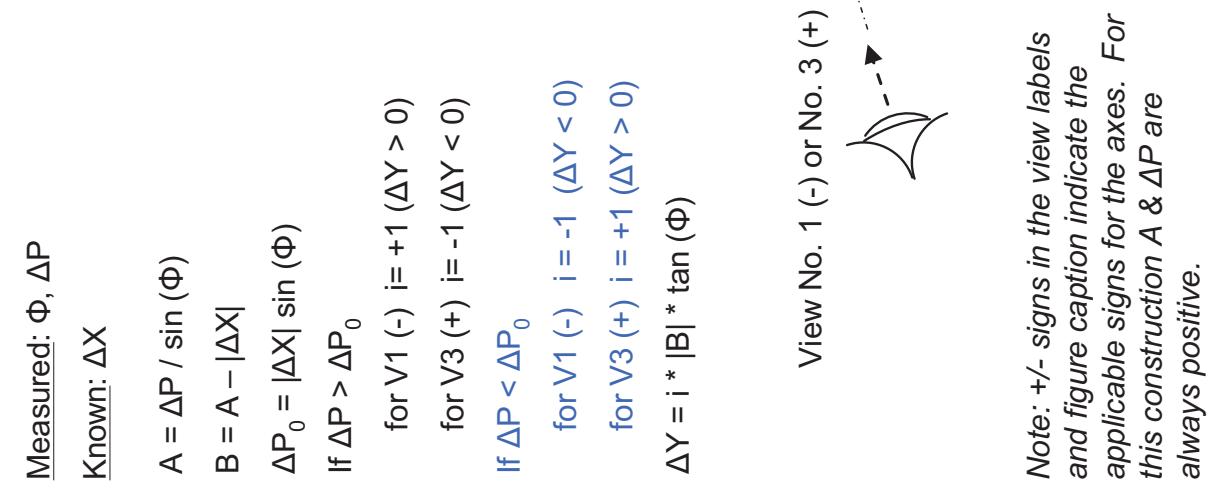

분 


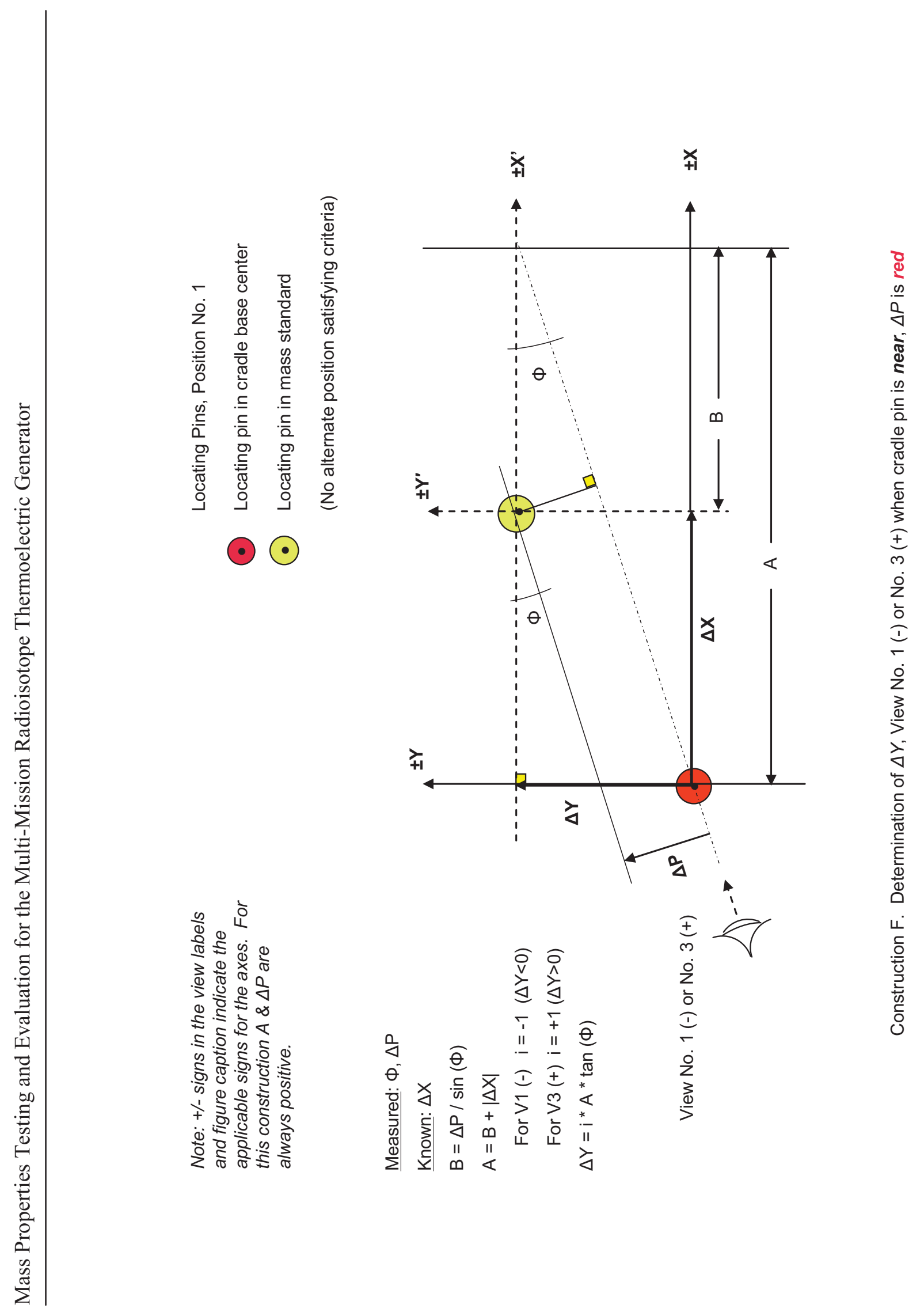



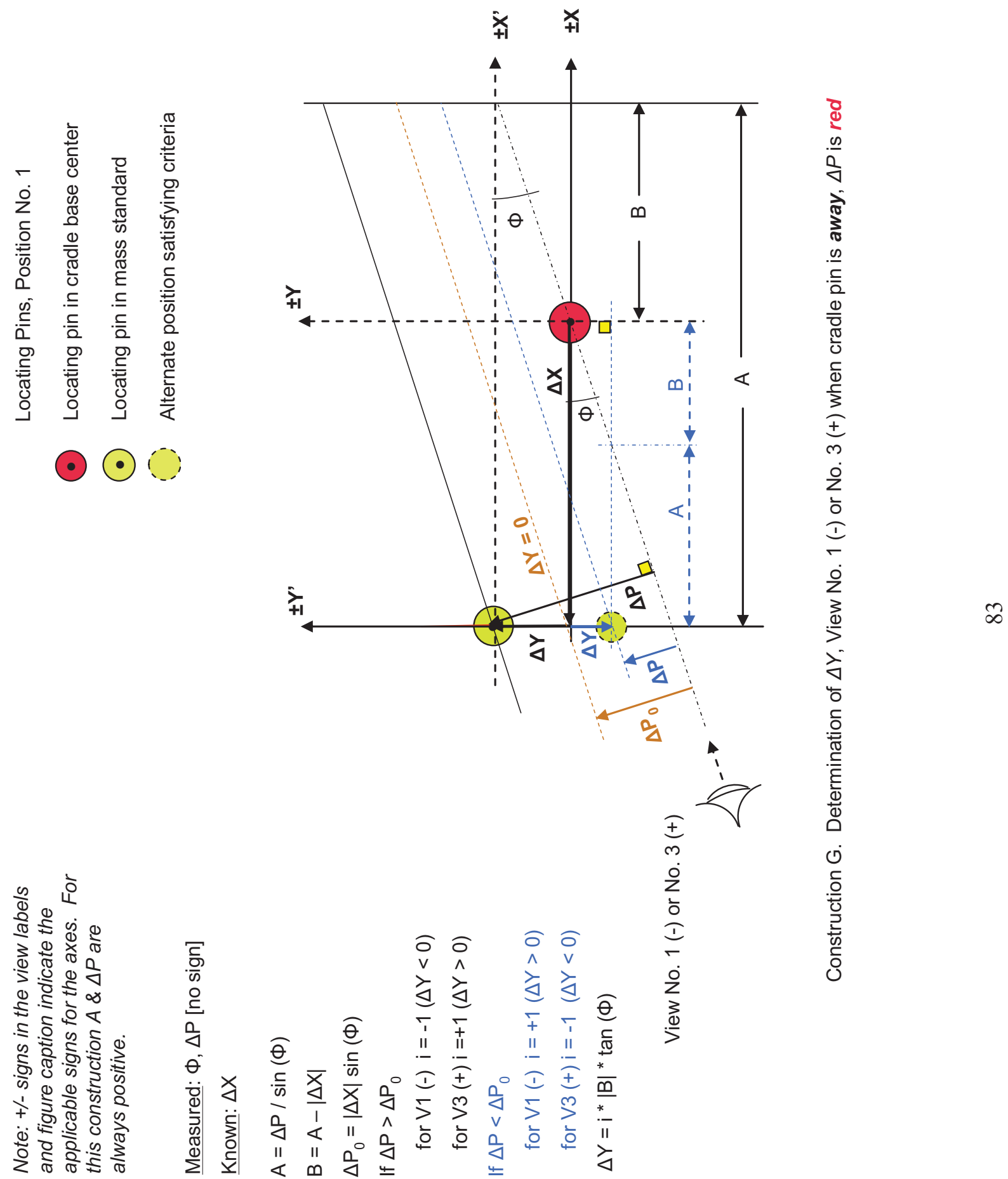


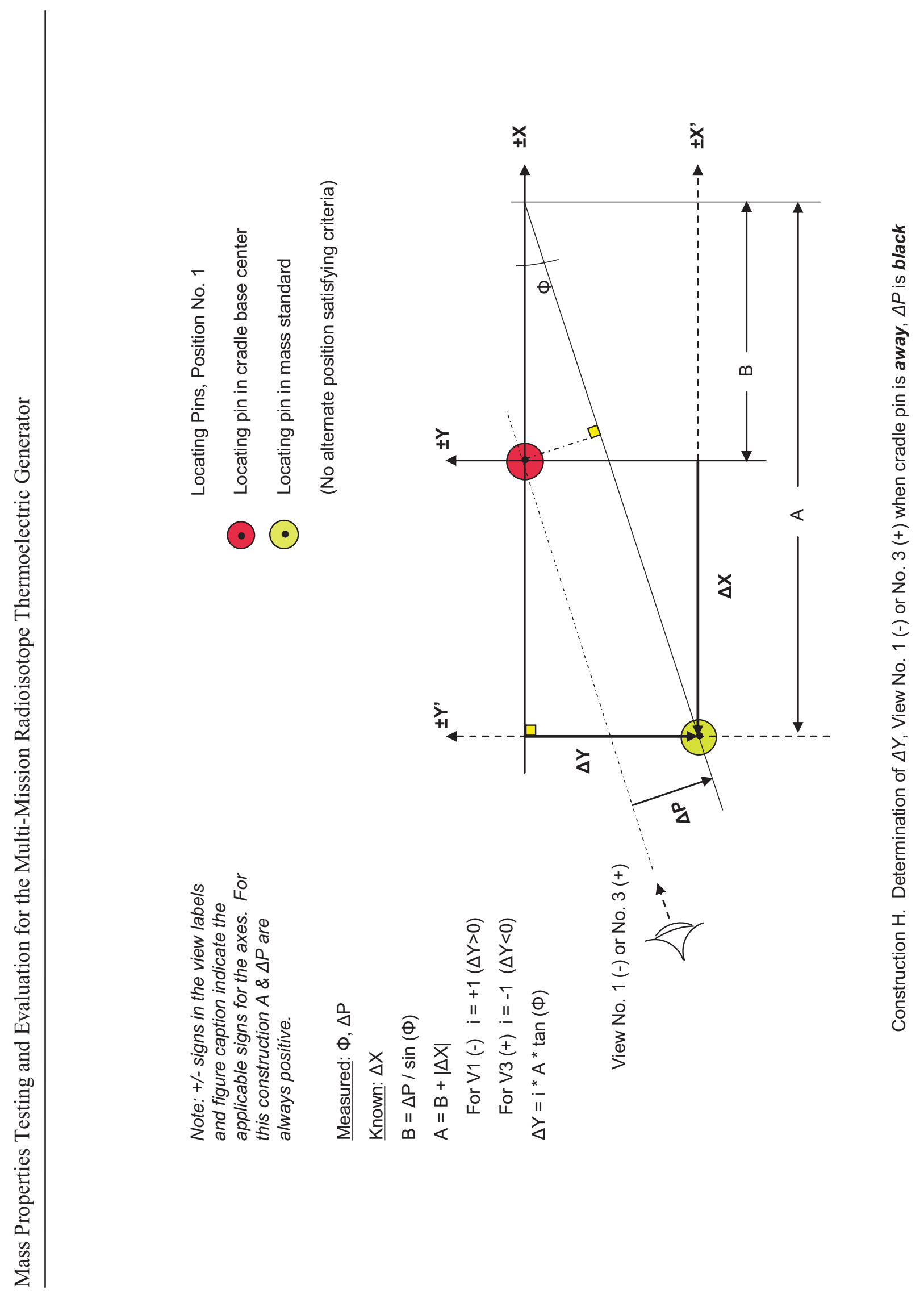




\section{Appendix C}

\section{Specific Measurement System Output - Mass Standard}




\section{Appendix C Specific Measurement System Output - Mass Standard}

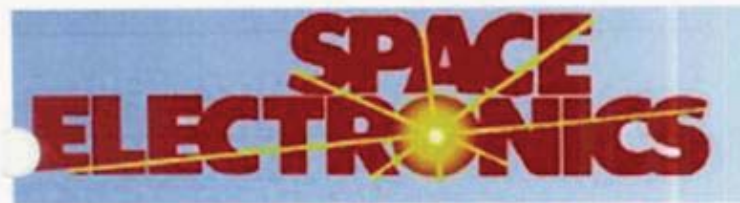

Model : KSR1320-1500

Version : 4.2 .3

Serial : $2938 / 72818$

\section{RunNumber : 001614}

Date : July 2, 2009

Time: 10:25

Operator: Browning

Part ID: Mass simulator

Part Serial : Position 1 run 2

Comments: comparison measurement for the following

- sensitioity measurement.

\section{CG Part Measurement Results}

Moment Readings

\begin{tabular}{|l|r|r|r|}
\hline & $\begin{array}{r}\text { Transducer Reading } \\
\text { (counts) }\end{array}$ & $\begin{array}{r}\text { Transducer Moment } \\
\text { (lb-in) }\end{array}$ & $\begin{array}{c}\text { Reading Angle } \\
\text { (deg) }\end{array}$ \\
\hline Reading Position 1: & 60592.8689 & 2076.875 & 0 \\
\hline Reading Position 2: & 60610 & 2077.462 & 90 \\
\hline Reading Position 3: & 60589 & 2076.743 & 180 \\
\hline Reading Position 4: & 60576,9836 & 2076.331 & 270 \\
\hline
\end{tabular}

This is a 4 position measurement.
Suann beller

$7 / 2 / 09$ 


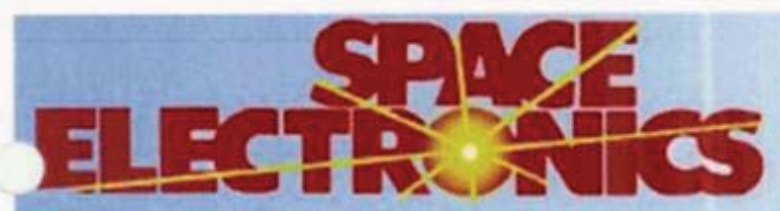

Model: KSR1320-1500

Version : 4.2.3

Serial : $2938 / 72818$
RunNumber: 001615

Date : July 2, 2009

Time: 10:39

Operator: Browning

Part ID : Mass simulator

Part Serial: Position 1

Comments : sensitivity measurement with pin in hole $\mathrm{H}$ (+Xaxis)

\section{CG Part Measurement Results}

\section{Moment Readings}

\begin{tabular}{|l|r|r|r|}
\hline & $\begin{array}{r}\text { Transducer Reading } \\
\text { (counts) }\end{array}$ & $\begin{array}{r}\text { Transducer Moment } \\
\text { (lb-in) }\end{array}$ & $\begin{array}{c}\text { Reading Angle } \\
\text { (deg) }\end{array}$ \\
\hline Reading Position 1 : & 60690.7869 & 2080.231 & 0 \\
\hline Reading Position 2: & 60608 & 2077.394 & 180 \\
\hline Reading Position 3: & 60492.0656 & 2073.420 & 270 \\
\hline Reading Position 4: & 60573.9836 & 2076.228 & Jue Ann jocller \\
\hline
\end{tabular}




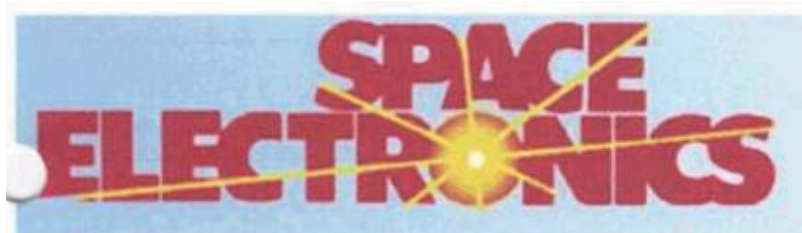

Model : KSR1320-1500

Version : 4.2 .3

Serial : $2938 / 72818$
RunNumber : 001616

Date : July 2, 2009

Time: 10:48

Operator: Browning

Part ID : Mass simulator

Part Serial : Position 1

Comments : sensitivity measurement with pin in hole I (-Xaxis)

\section{CG Part Measurement Results}

Moment Readings

\begin{tabular}{|r|r|r|r|}
\hline & $\begin{array}{c}\text { Transducer Reading } \\
\text { (counts) }\end{array}$ & $\begin{array}{c}\text { Transducer Moment } \\
\text { (lb-in) }\end{array}$ & $\begin{array}{c}\text { Reading Angle } \\
\text { (deg) }\end{array}$ \\
\hline Reading Position 1: & 60494.5902 & 2073.507 & 0 \\
\hline Reading Position 2: & 60605 & 2077.291 & 90 \\
\hline Reading Position 3: & 60687 & 2080.102 & 180 \\
\hline Reading Position 4: & 60571.9836 & 2076.159 & 270 \\
\hline
\end{tabular}

This is a 4 position measurement.

$$
\begin{gathered}
\text { guann ffiler } \\
7 / 2 \log
\end{gathered}
$$




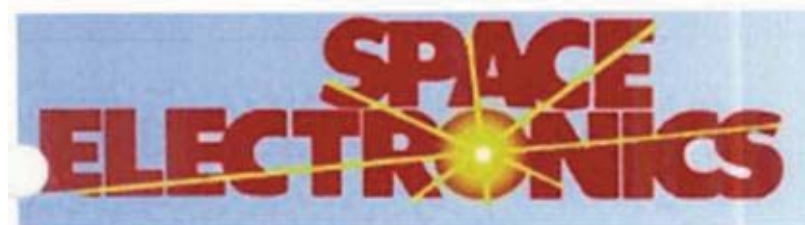

Model: KSR1320-1500

Version : 4.2.3

Serial : $2938 / 72818$
RunNumber: 001617

Date: July 2, 2009

Time: 10:58

Operator: Browning

Part ID : Wass simulator

Part Serial : Position 1

Comments : sensitivity measurement with pin in hole $A(+Y$ axis)

\section{CG Part Measurement Results}

\section{Moment Readings}

\begin{tabular}{|c|r|r|r|}
\hline & $\begin{array}{r}\text { Transducer Reading } \\
\text { (counts) }\end{array}$ & $\begin{array}{c}\text { Transducer Moment } \\
\text { (lb-in) }\end{array}$ & $\begin{array}{c}\text { Reading Angle } \\
\text { (deg) }\end{array}$ \\
\hline Reading Position 1 : & 60589.8689 & 2076.772 & 0 \\
\hline Reading Position 2: & 60703.082 & 2080.653 & 90 \\
\hline Reading Position 3 : & 60586 & 2076.640 & 180 \\
\hline Reading Position 4: & 60476.1803 & 2072.876 & 270 \\
\hline
\end{tabular}

This is a 4 position measurement.

\section{Excessive Variation : False}

$$
\text { jueam beller }
$$




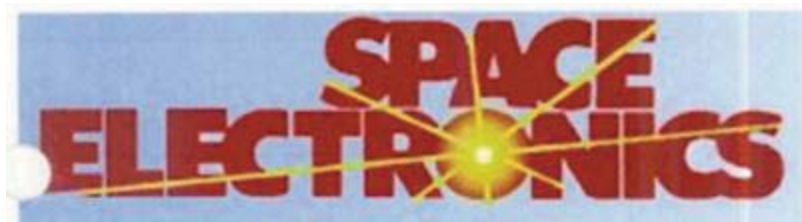

Model: KSR1320-1500

Version : 4.2 .3

Serial : $2938 / 72818$
RunNumber : 001618

Date : July 2, 2009

Time: 11:07

Operator: Browning

Part ID : Mass simulator

Part Serial : Position 1

Comments : sensitivity measurement with pin in hole $G(-Y$ axis)

\section{CG Part Measurement Results}

\section{Moment Readings}

\begin{tabular}{|c|r|r|r|}
\hline & $\begin{array}{c}\text { Transducer Reading } \\
\text { (counts) }\end{array}$ & $\begin{array}{r}\text { Transducer Moment } \\
\text { (lb-in) }\end{array}$ & $\begin{array}{c}\text { Reading Angle } \\
\text { (deg) }\end{array}$ \\
\hline Reading Position 1: & 60589.9836 & 2076.776 & 0 \\
\hline Reading Position 2 : & 60506 & 2073.898 & 90 \\
\hline Reading Position 3 : & 60588 & 2076.708 & 180 \\
\hline Reading Position 4 : & 60669 & 2079.485 & 270 \\
\hline
\end{tabular}

This is a 4 position measurement.

\section{Excessive Variation: False}

$$
\text { buann bether }
$$$$
7 / 2 / 09
$$ 


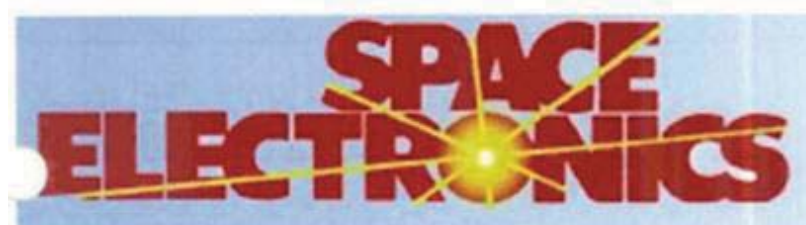

Model: KSR1320-1500

Version : 4.2 .3

Serial : $2938 / 72818$
RunNumber : 001619

Date : July 2, 2009

Time: 13:02

Operator: Browning

Tare ID 1: Fixture Tare after simulator removed

Tare ID 2: Position 1

Comments : applys to runs 1614-1618

\section{CG Tare Measurement Results}

\section{Moment Readings}

\begin{tabular}{|r|r|r|r|}
\hline & $\begin{array}{r}\text { Transducer Reading } \\
\text { (counts) }\end{array}$ & $\begin{array}{r}\text { Transducer Moment } \\
\text { (lb-in) }\end{array}$ & $\begin{array}{c}\text { Reading Angle } \\
\text { (deg) }\end{array}$ \\
\hline Reading Position 1 : & 60573 & 2076.194 & 0 \\
\hline Reading Position 2: & 60586 & 2076.640 & 90 \\
\hline Reading Position 3: & 60577.0492 & 2076.333 & 180 \\
\hline Reading Position 4: & 60569 & 2076.057 & 270 \\
\hline
\end{tabular}

This is a 4 position measurement. 


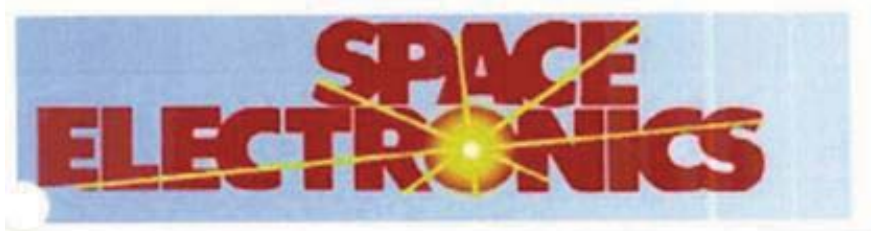

Model : KSR1320-1500

Version: 4.2 .3

Serial : $2938 / 72818$
RunNumber : 001620

Date : July 2, 2009

Time : 13:11

Operator: Browning

Part ID : MS CG Calculation

Part Serial : Position 1

Comments : Base calculation

\section{Calculation Data}

CG Tare : 001619 July 2, 2009

CG Part : 001614 July 2, 2009

Part Weight: $\quad 97.1980 \quad$ lb
13:02

10:25
Fixture Tare after simulator removed Mass simulator

Part Estimated CG Height : 26.1000 in

Machine Angle at Part 0 Degrees : $\quad 0.000$ deg

User Angle System Polarity: Positive (CCW INCREASING ANGLE)

User Datums Offset below are in Machine Angle System

User Datum Offset X : $\quad-\quad 0.0030$ in User Datum Offset $Y$ : $\quad 0.0030$ in

\section{Calculation Results}

Calculated Static CG (see Note 1)

\begin{tabular}{|r|r|r|}
\hline $\begin{array}{l}\text { Machine } \\
\text { Referenced }\end{array}$ & $\begin{array}{c}\text { CG Offset } \\
\text { (in) }\end{array}$ & $\begin{array}{c}\text { CG Moment } \\
\text { (lb-in) }\end{array}$ \\
\hline Static CG X : & $\mathbf{0 . 0 0 1 4}$ & $\mathbf{0 . 1 3 5}$ \\
\hline Static CG Y : & $\mathbf{0 . 0 0 2 8}$ & $\mathbf{0 . 2 7 5}$ \\
\hline Static CG Mag : & $\mathbf{0 . 0 0 3 1}$ & $\mathbf{0 . 3 0 6}$ \\
\hline Static CG Angle : & $\mathbf{6 3 . 8 8 5}$ & $\mathbf{6 3 . 8 8 5}$ \\
\hline
\end{tabular}

\begin{tabular}{|r|r|r|}
\hline $\begin{array}{l}\text { User } \\
\text { Referenced }\end{array}$ & $\begin{array}{c}\text { CG Offset } \\
\text { (in) }\end{array}$ & $\begin{array}{r}\text { CG Moment } \\
\text { (lb-in) }\end{array}$ \\
\hline Static CG X : & $\mathbf{0 . 0 0 4 4}$ & $\mathbf{0 . 4 2 6}$ \\
\hline Static CG Y : & $-\mathbf{0 . 0 0 0 2}$ & -0.017 \\
\hline Static CG Mag : & $\mathbf{0 . 0 0 4 4}$ & $\mathbf{0 . 4 2 7}$ \\
\hline Static CG Angle : & 357.717 & 357.717 \\
\hline
\end{tabular}

Suann Keller

$712 / 09$

Notes

1. CG Uncertainty

CG Moment Uncertainty

CG Offset Uncertainty

$0.169 \mathrm{lb}$-in

0.0017 in 


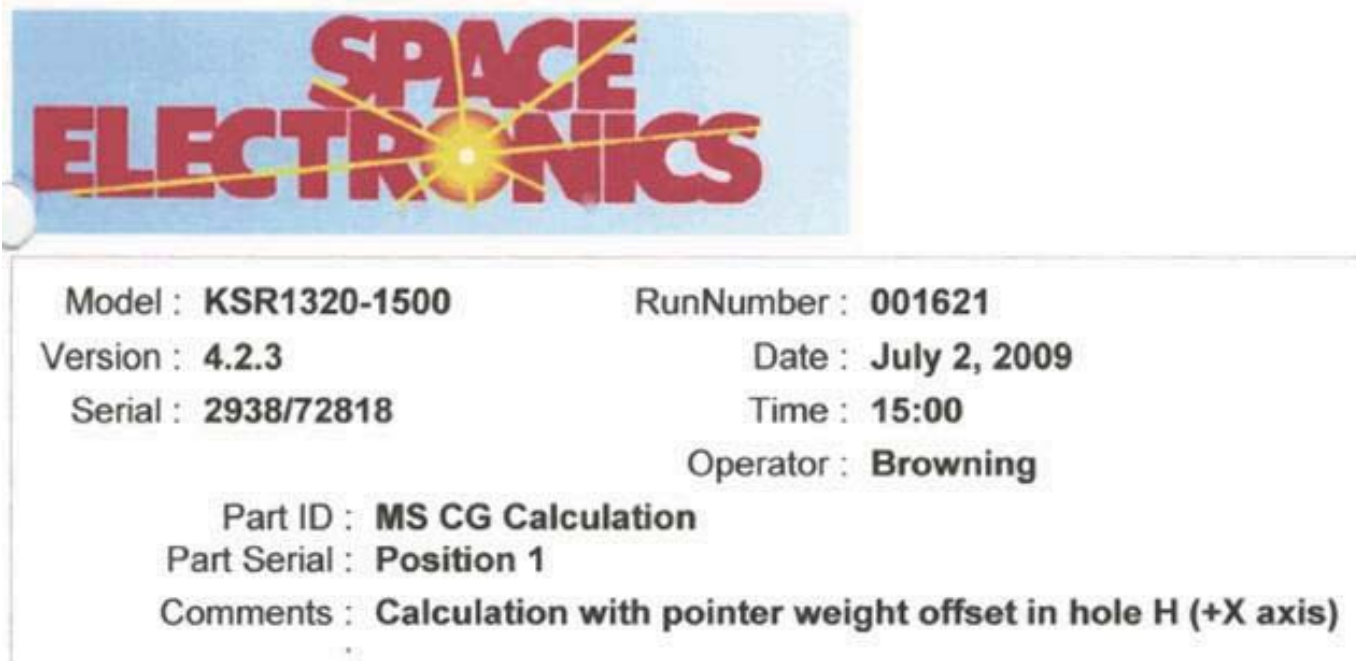

\section{Calculation Data}

CG Tare : 001619 July 2, 2009

CG Part : 001615 July 2, 2009

Part Weight : $\quad 97.5299 \quad \mathrm{lb}$
13:02

10:39

Part Estimated CG Height : Mass simulator
Fixture Tare after simulator removed

26.1000 in

Machine Angle at Part 0 Degrees : $\quad 0.000$ deg

User Angle System Polarity : Positive (CCW INCREASING ANGLE)

User Datums Offset below are in Machine Angle System

User Datum Offset X: $\quad-\mathbf{0 . 0 0 3 0}$ in $\quad$ User Datum Offset $Y$ : $\quad \mathbf{0 . 0 0 3 0}$ in

\section{Calculation Results}

Calculated Static CG (see Note 1)

\begin{tabular}{|c|c|c|c|c|c|}
\hline \\
\hline $\begin{array}{l}\text { Machine } \\
\text { Referenced }\end{array}$ & $\begin{array}{l}\text { CG Offset } \\
\text { (in) }\end{array}$ & $\begin{array}{l}\text { CG Moment } \\
(\mathrm{lb}-\mathrm{in})\end{array}$ & $\begin{array}{l}\text { User } \\
\text { Referenced }\end{array}$ & $\begin{array}{l}\text { CG Offset } \\
\text { (in) }\end{array}$ & $\begin{array}{l}\text { CG Moment } \\
\text { (lb-in) }\end{array}$ \\
\hline Static CG X : & 0.0356 & 3.470 & Static CG X : & 0.0386 & 3.762 \\
\hline Static CG Y : & 0.0031 & 0.303 & Static CG Y : & 0.0001 & 0.010 \\
\hline Static CG Mag : & 0.0357 & 3.483 & Static CG Mag : & 0.0386 & 3.762 \\
\hline Static CG Angle : & 4.988 & 4.988 & Static CG Angle : & 0.156 & 0.156 \\
\hline \multicolumn{6}{|c|}{ bue ann beller } \\
\hline \multicolumn{2}{|c|}{$7 / 2 / 09$} & & $\begin{array}{l}\text { Notes } \\
\text { 1. CG Uncertainty } \\
\text { CG Moment Uncer } \\
\text { CG Offset Uncertai }\end{array}$ & ainty & $\begin{array}{l}173 \mathrm{lb} \text {-in } \\
1018 \mathrm{in}\end{array}$ \\
\hline
\end{tabular}




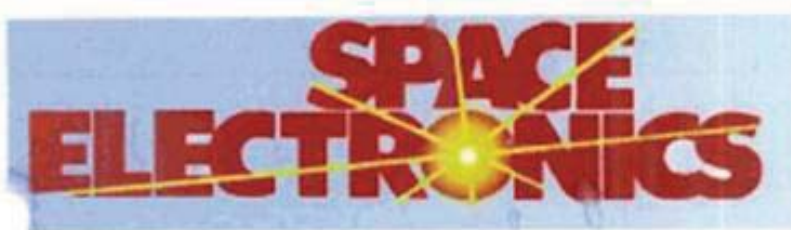

Model: KSR1320-1500

RunNumber: 001622

Version : 4.2 .3

Date : July 2, 2009

Serial : 293872818

Time: 15:01

Operator: Browning

Part ID : MS CG Calculation

Part Serial : Position 1

Comments : Calculation with pointer weight offset in hole I (-X axis)

\section{Calculation Data}

$\begin{array}{llll}\text { CG Tare : } 001619 & \text { July 2, 2009 } & 13: 02 & \text { Fixture Tare after simulator removed } \\ \text { CG Part : 001616 } & \text { July 2, 2009 } & 10: 48 & \text { Mass simulator }\end{array}$

Part Weight: $\mathbf{9 7 . 5 2 9 9} \mathrm{lb} \quad$ Part Estimated CG Height : $\mathbf{2 6 . 1 0 0 0}$ in

Machine Angle at Part 0 Degrees : $\quad 0.000$ deg

User Angle System Polarity : Positive (CCW INCREASING ANGLE)

User Datums Offset below are in Machine Angle System
User Datum Offset $X$ :
-0.0030 in
User Datum Offset $Y$ :
0.0030 in

Calculation Results Calculated Static CG (see Note 1)

\begin{tabular}{|r|r|r|}
\hline $\begin{array}{l}\text { Machine } \\
\text { Referenced }\end{array}$ & $\begin{array}{c}\text { CG Offset } \\
\text { (in) }\end{array}$ & $\begin{array}{c}\text { CG Moment } \\
\text { (lb-in) }\end{array}$ \\
\hline Static CG X: & -0.0331 & -3.225 \\
\hline Static CG Y : & 0.0027 & 0.263 \\
\hline Static CG Mag : & 0.0332 & 3.236 \\
\hline Static CG Angle : & 175.331 & 175.331 \\
\hline Sue Onn jeller & \\
\hline $7 / 2 / 09$ &
\end{tabular}

\begin{tabular}{r|r|r|}
\hline $\begin{array}{l}\text { User } \\
\text { Referenced }\end{array}$ & $\begin{array}{c}\text { CG Offset } \\
\text { (in) }\end{array}$ & $\begin{array}{c}\text { CG Moment } \\
\text { (lb-in) }\end{array}$ \\
\hline Static CG X: & -0.0301 & -2.932 \\
\hline Static CG Y : & -0.0003 & -0.029 \\
\hline Static CG Mag : & 0.0301 & 2.933 \\
\hline Static CG Angle : & 180.571 & 180.571 \\
\hline
\end{tabular}

\section{Notes}

1. CG Uncertainty

CG Moment Uncertainty CG Offset Uncertainty

$0.172 \mathrm{lb}$-in 0.0018 in 


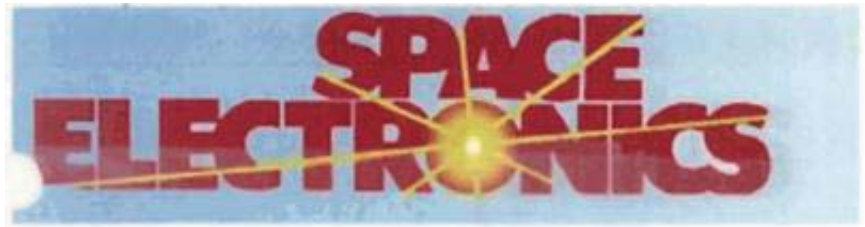

Model : KSR1320-1500

Version : 4.2 .3

Serial : $2938 / 72818$
RunNumber: 001623

Date: July 2, 2009

Time: 15:02

Operator: Browning

Part ID : MS CG Calculation

Part Serial : Position 1

Comments : Calculation with pointer weight offset in hole $A(+Y$ axis)

\section{Calculation Data}

CG Tare : 001619 July 2, 2009

CG Part : 001617 July 2, 2009

Part Weight: $\quad 97.5299 \quad \mathrm{lb}$
13:02

10:58
Fixture Tare after simulator removed Mass simulator

Part Estimated CG Height : $\quad 26.1000$ in

Machine Angle at Part 0 Degrees : $\mathbf{0 . 0 0 0}$ deg

User Angle System Polarity : Positive (CCW INCREASING ANGLE)

idl User Datums Offset below are in Machine Angle System
User Datum Offset X :
-0.0030 in
User Datum Offset $Y$ :
$\mathbf{0 . 0 0 3 0}$ in

\section{Calculation Results}

Calculated Static CG (see Note 1)

\begin{tabular}{|c|c|c|c|c|c|}
\hline \\
\hline $\begin{array}{l}\text { Machine } \\
\text { Referenced }\end{array}$ & $\begin{array}{l}\text { CG Offset } \\
\text { (in) }\end{array}$ & $\begin{array}{c}\text { CG Moment } \\
\text { (lb-in) }\end{array}$ & $\begin{array}{l}\text { User } \\
\text { Referenced }\end{array}$ & $\begin{array}{l}\text { CG Offset } \\
\text { (in) }\end{array}$ & $\begin{array}{l}\text { CG Moment } \\
\text { (lb-in) }\end{array}$ \\
\hline Static CG X: & 0.0013 & 0.124 & Static CG X : & 0.0043 & 0.416 \\
\hline Static CG Y : & 0.0368 & 3.593 & Static CG Y : & 0.0338 & 3.301 \\
\hline Static CG Mag : & 0.0369 & 3.595 & Static CG Mag : & 0.0341 & 3.327 \\
\hline Static CG Angle : & 88.031 & 88.031 & Static CG Angle : & 82.814 & 82.814 \\
\hline \multicolumn{6}{|c|}{ Sue ann belles } \\
\hline $7 / 2 / 09$ & & & \multicolumn{3}{|l|}{$\begin{array}{l}\text { Notes } \\
\text { 1. CG Uncertainty }\end{array}$} \\
\hline & & & \multicolumn{2}{|c|}{$\begin{array}{l}\text { CG Moment Uncertainty } \\
\text { CG Offset Uncertainty }\end{array}$} & $\begin{array}{l}0.173 \mathrm{lb} \text {-in } \\
.0018 \mathrm{in}\end{array}$ \\
\hline
\end{tabular}




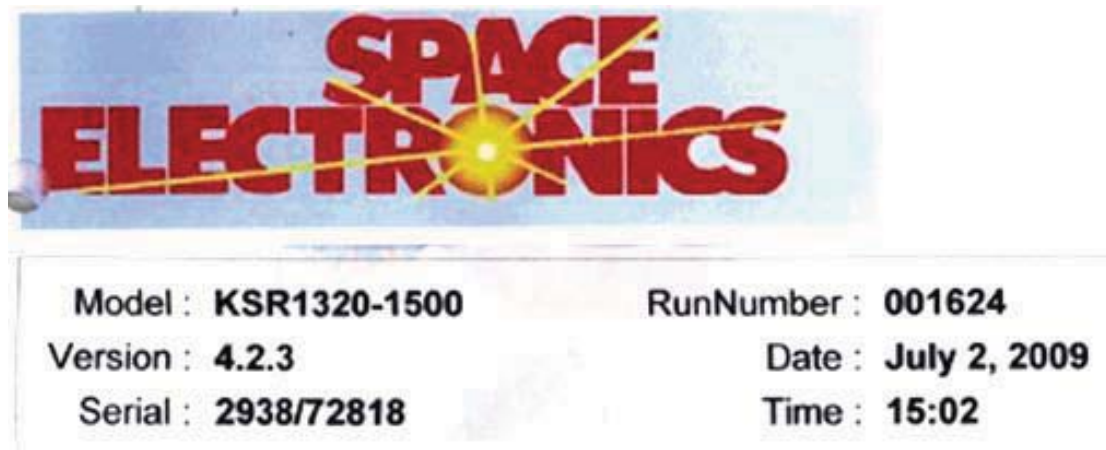

\section{Part Serial: Position 1}

Comments : Calculation with pointer weight offset in hole G (-Y axis)

\section{Calculation Data}

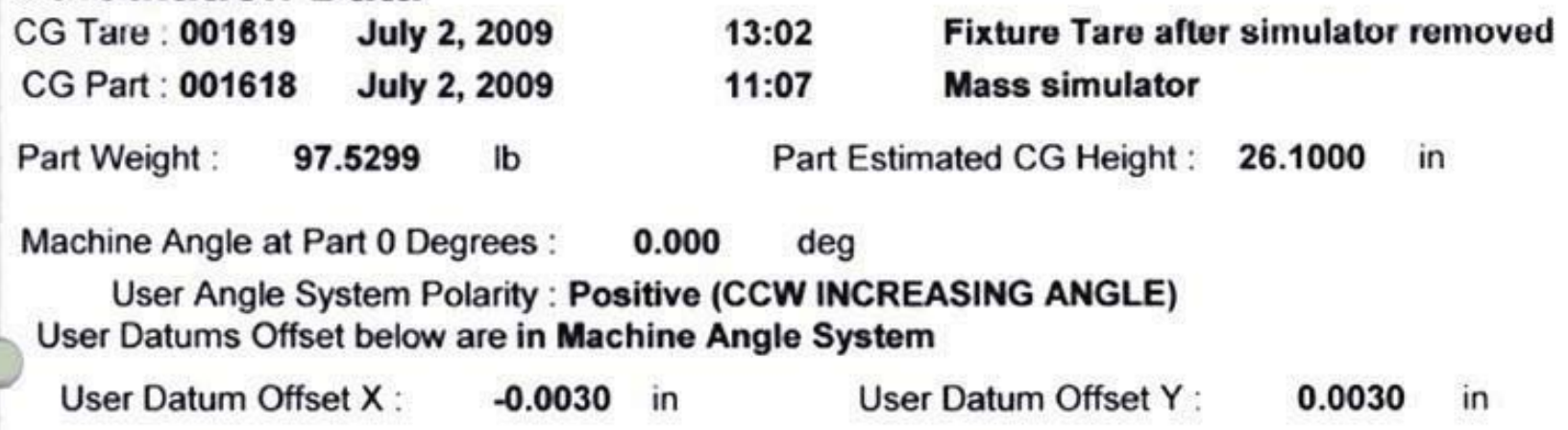

\section{Calculation Results} Calculated Static CG (see Note 1)

\begin{tabular}{|c|c|c|c|c|c|}
\hline $\begin{array}{l}\text { Machine } \\
\text { Referenced }\end{array}$ & $\begin{array}{l}\text { CG Offset } \\
\text { (in) }\end{array}$ & $\begin{array}{l}\text { CG Moment } \\
\text { (lb-in) }\end{array}$ & $\begin{array}{l}\text { User } \\
\text { Referenced }\end{array}$ & $\begin{array}{l}\text { CG Offset } \\
\text { (in) }\end{array}$ & $\begin{array}{c}\text { CG Moment } \\
\text { (lb-in) }\end{array}$ \\
\hline Static CG X: & 0.0012 & 0.114 & Static CG X: & 0.0042 & 0.406 \\
\hline Static CGY: & -0.0316 & -3.081 & Static CG Y : & -0.0346 & -3.373 \\
\hline Static CG Mağ : & 0.0316 & 3.083 & Static CG Mag : & 0.0348 & 3.398 \\
\hline Static CG Angle: & 272.111 & 272.111 & Static CG Angle : & 276.865 & 276.865 \\
\hline \multicolumn{6}{|c|}{ buann beller. } \\
\hline
\end{tabular}




\section{Appendix D}

\section{Fixture Temperature Maps}




\section{Appendix D Fixture Temperature Maps}

Note: Temperature maps in this appendix were copied from the actual MMRTG test procedure, RPS-OI-28220. The header information and Appendix designator (K) shown on these maps pertain to the test procedure document, not to the present document.

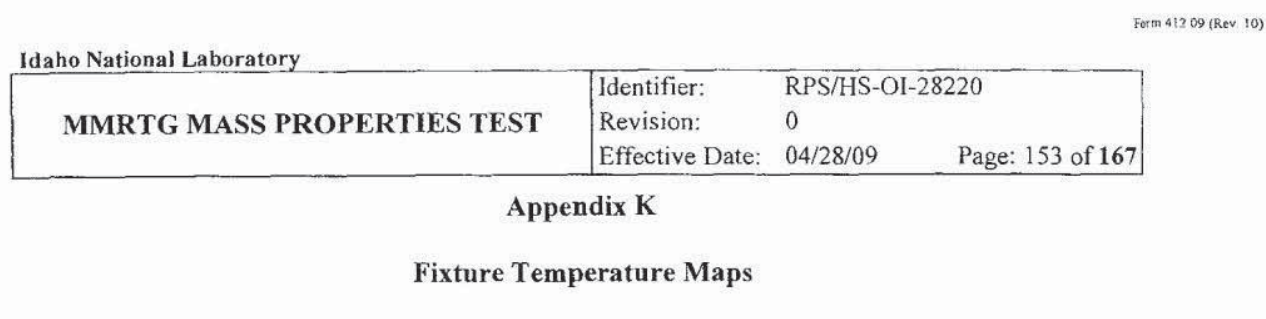

\section{1st Attempt}

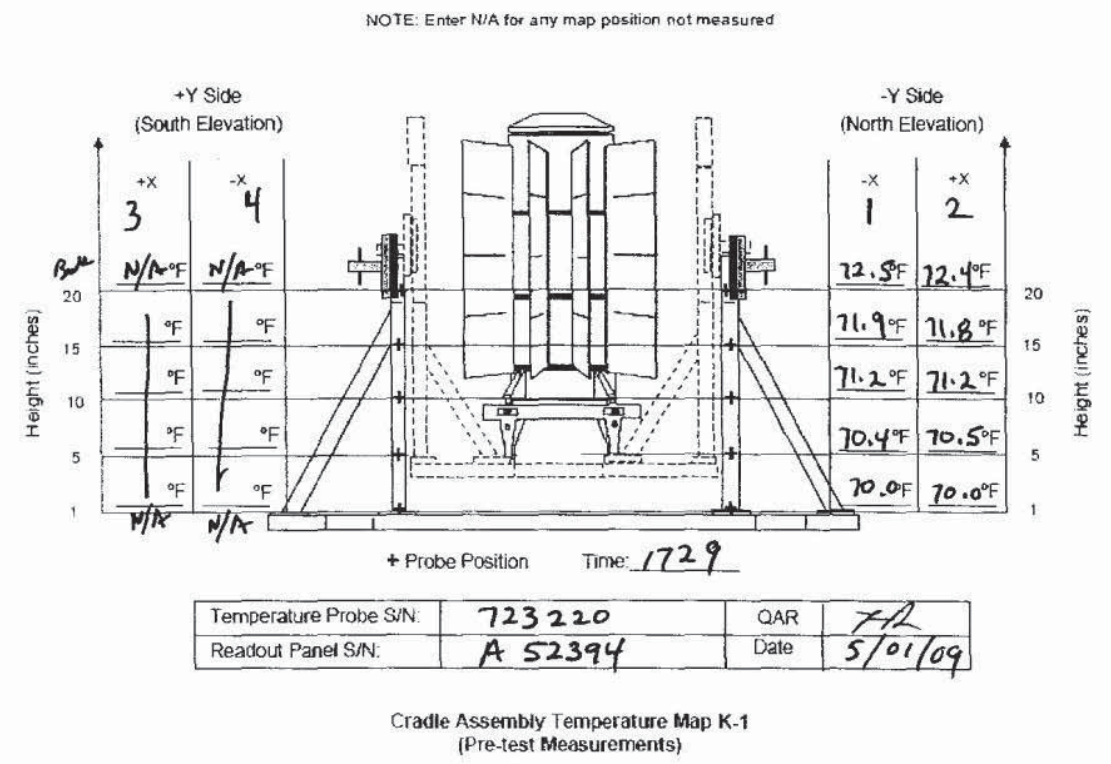

N/A pg

Appendix K 
Form 412.09 (Rev. 10)

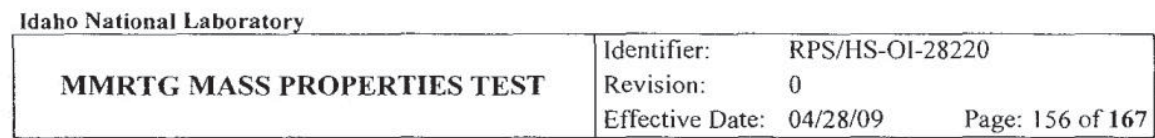

\section{1st Attempt}

NOTE: Enter N/A for any map position not measured

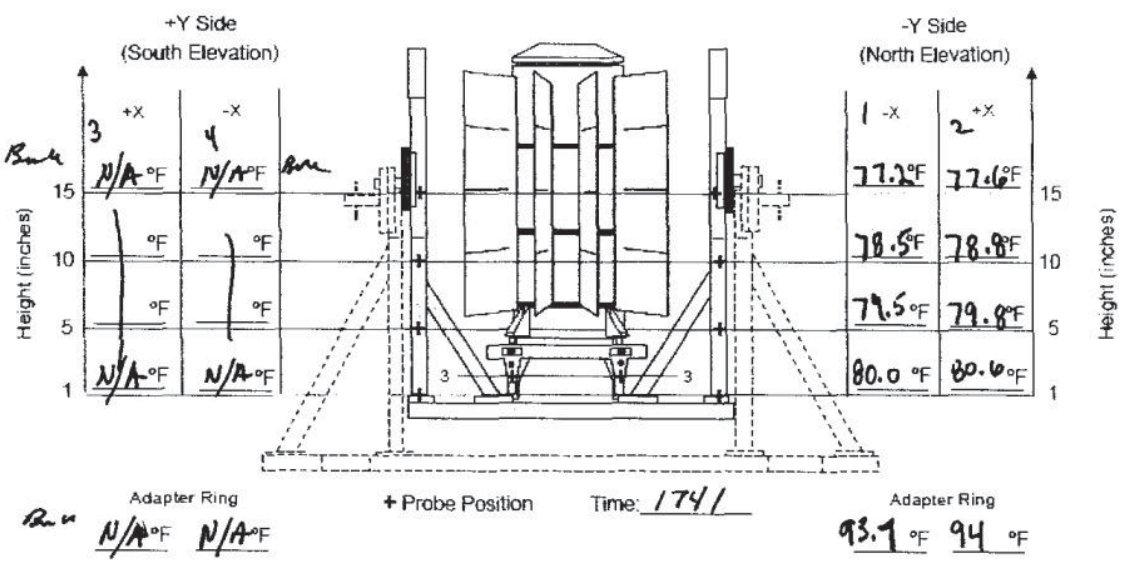

Rotation Fixture Assembly Temperature Map K-2 (Pre-test Measurements)

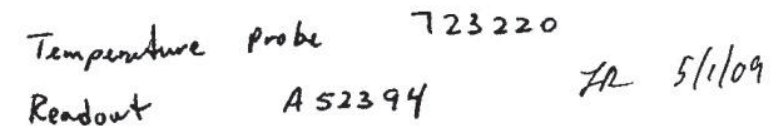




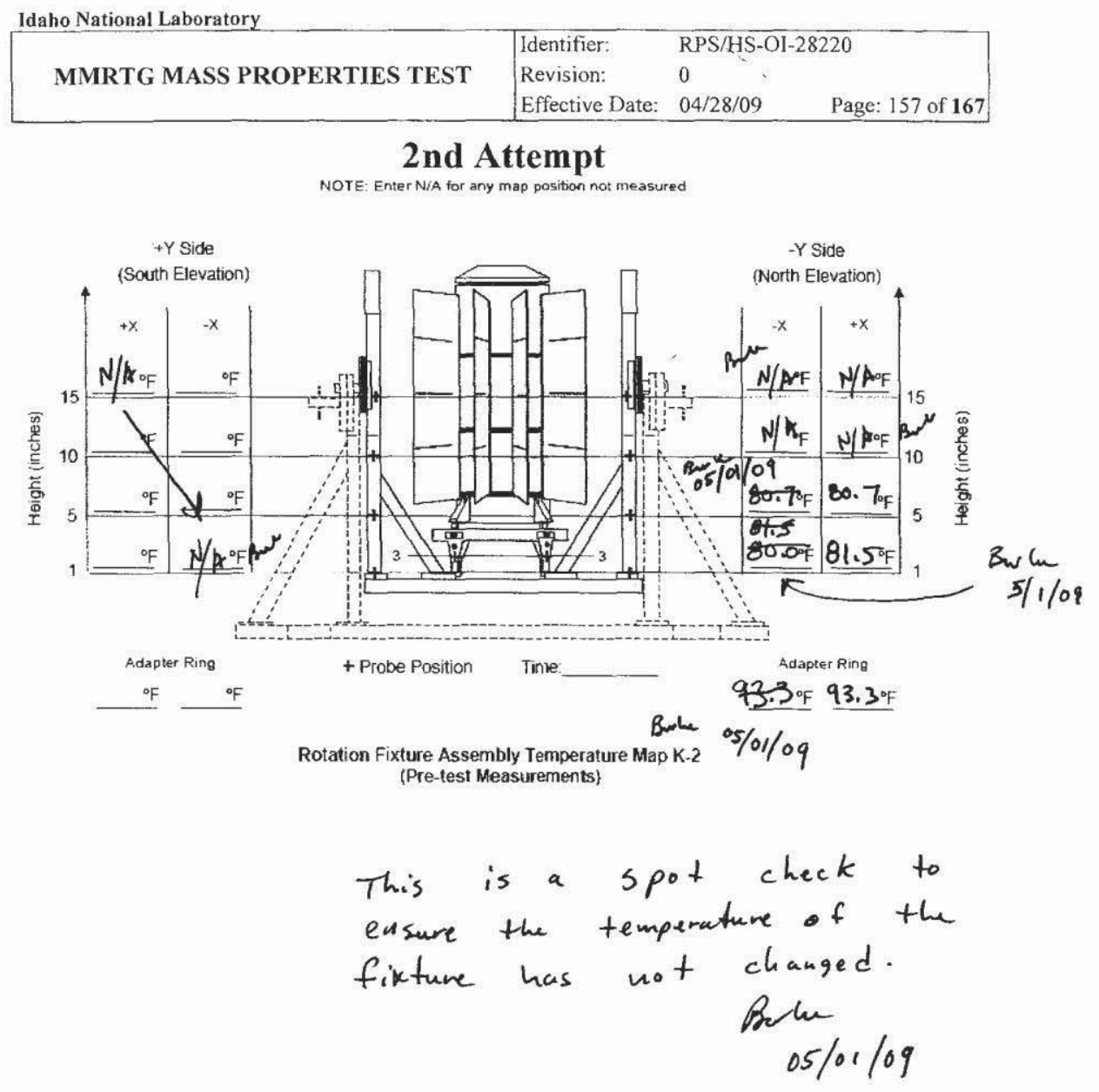


Forrs 41209 (Rev. 10)

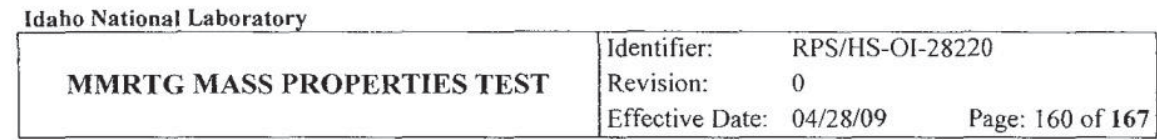

1st Attempt

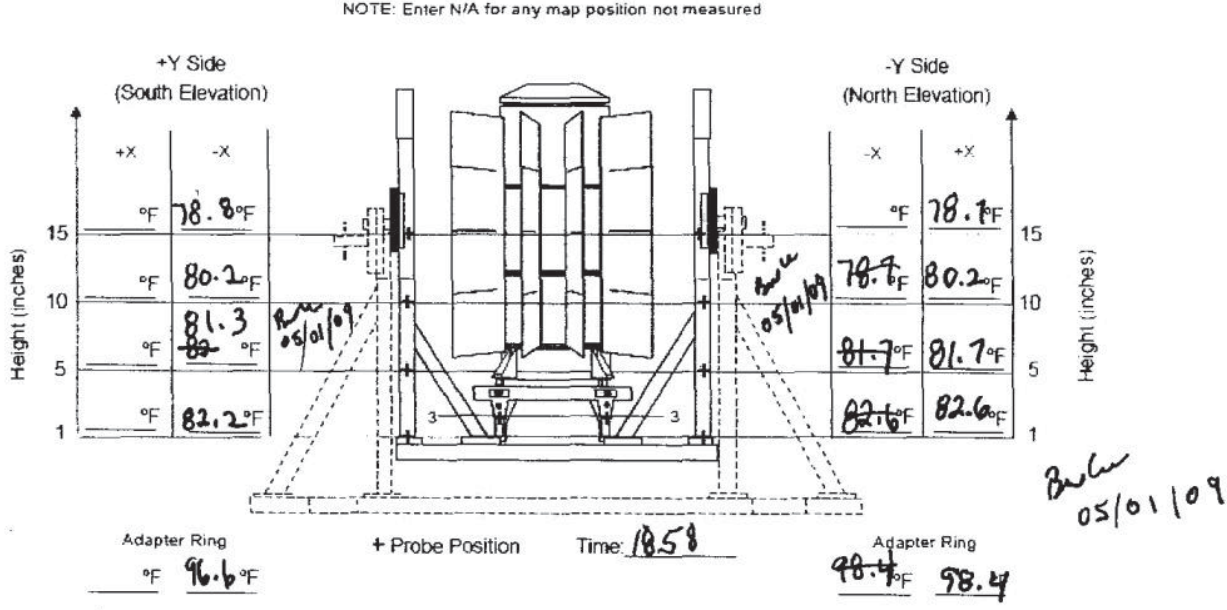

Rotation Fixture Assembly Temperature Map K-4

(Post-test Measurements) 
Form 41209 (Rev. 10)

Idaho National Laboratory
\begin{tabular}{|c|lll|}
\hline & Identifier: & RPS/HS-OI-28220 & \\
MMRTG MASS PROPERTIES TEST & Revision: & 0 & Page: 162 of 167 \\
\hline
\end{tabular}

1st Attempt

NOTE: Enter NiA for any map position not measured

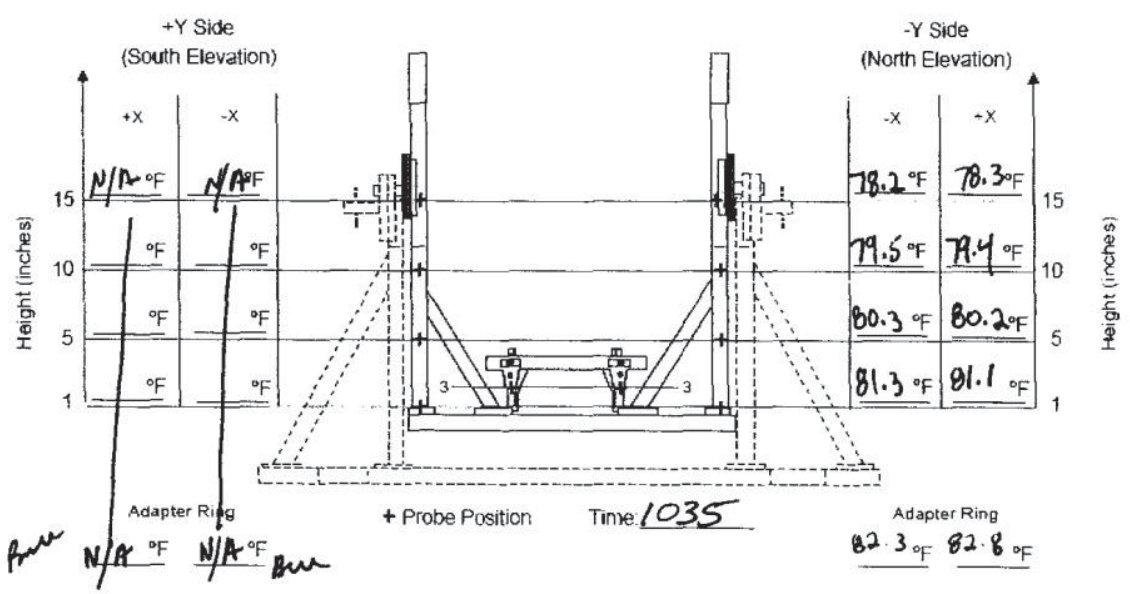

Rotation Fixture Assembly Temperature Map K-6

(Pre-Tare Temperatures)

STEP $5.12 \cdot 1 \cdot 3$ 


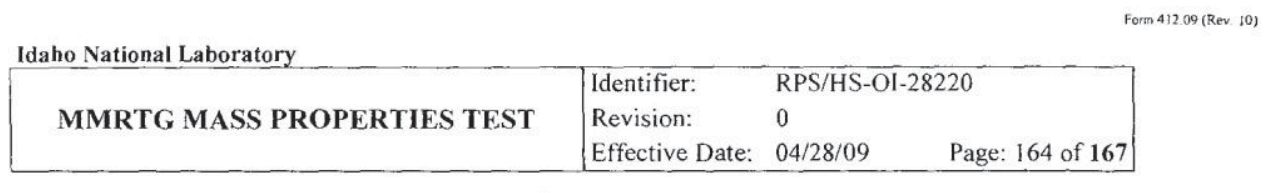

\section{1st Attempt}

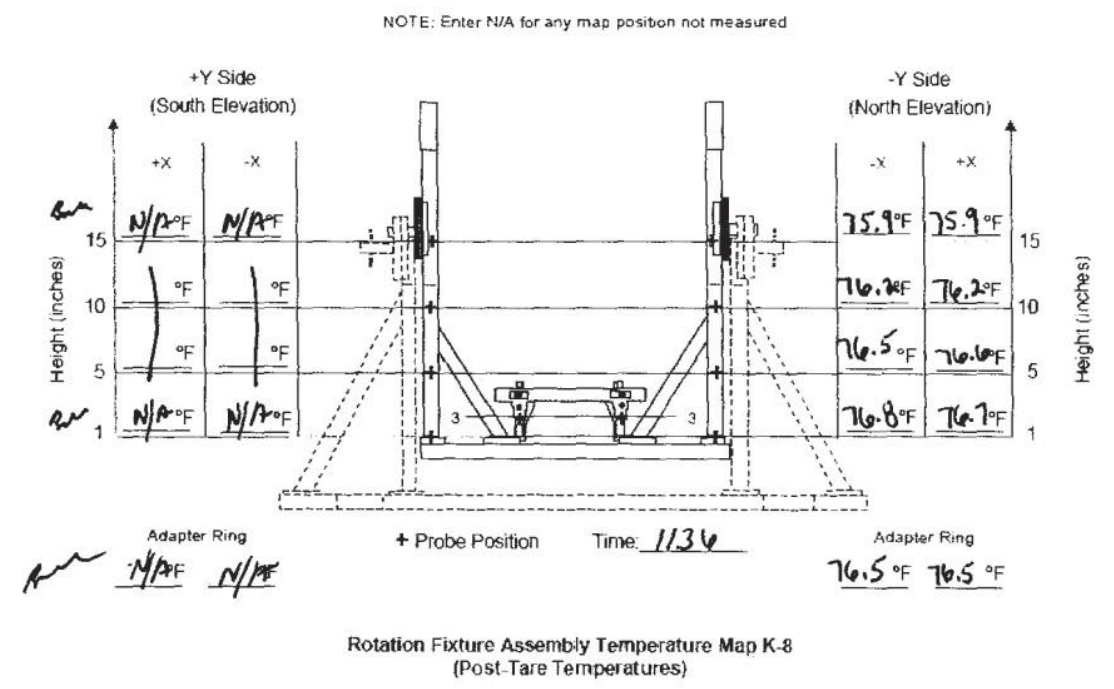

STEP $5 \cdot 12 \cdot 4 \cdot 2$ 
INTENTIONALLY BLANK 


\section{Appendix E}

\section{Temperature Induced CG Corrections}




\section{Appendix E Temperature Induced CG Corrections}

The data from the Pro-E model of the adapter ring and rotation fixture were used to determine the temperature induced corrections to the MMRTG CG position. An Excel spreadsheet was constructed and used to track the positions of components that comprise these fixtures. Two different approaches may be used that will yield the same result: (1) the direct temperature changes between the PART and TARE conditions for corresponding components can be used; or (2) calculations may be made separately for the PART and TARE conditions with the temperature changes referenced to the nominal room temperature conditions and the PART offset subtracted from the TARE offset. Although equivalent, the latter approach takes advantage of the nominal room temperature dimensions and is the approach that is used in this appendix. The validity of this approach is demonstrated by considering the defining relation for the corrected test article CG as was developed in Section 10.2:

$$
C G_{T A}=C G_{T A}^{\prime}+\frac{1}{W_{T A}} \sum_{j} W_{j} * \Delta Y_{j}
$$

Each of the $\Delta Y_{j}$ terms represents the difference in the positions of the $j^{\text {th }}$ component's CG during the TARE (primes) and the during the PART measurements, $\left(\mathrm{Y}^{\prime}-\mathrm{Y}\right)_{\mathrm{j}}$. However, each of these positions can also be referenced to the normal room temperature positions denoted by a 0 subscript as follows:

$$
\Delta Y=Y^{\prime}-Y=Y^{\prime}-Y_{0}+Y_{0}-Y=Y^{\prime}-Y_{0}-\left(Y-Y_{0}\right)=\Delta Y_{0}^{\prime}-\Delta Y_{0}
$$

Therefore, the test article CG position finally becomes

$$
C G_{T A}=C G_{T A}^{\prime}+\frac{1}{W_{T A}} \sum_{j} W_{j} *\left(\Delta Y_{0}^{\prime}-\Delta Y_{0}\right)_{j}
$$

1. Adapter Ring

The adapter ring calculations are shown in the first section of spreadsheet E-1 (Table 7). The Pro-E model has the datum for the adapter ring at the center of the plane of its mounting feet. All of the adapter ring components were accurately included as detailed in the spreadsheet.

The left side of the spreadsheet lists all of the adapter ring components together with their weights and their individual CG positions for the centerline coordinate as determined from the Pro-E design. Nominal design dimensions are assumed to be those that pertain to the components at the prevailing ambient temperature during testing. The component moment relative to the adapter ring datum are calculated from the weight and design CG position. Summing the moments and dividing by the total weight of the adapter ring yields the composite $C G$ position relative to the datum. The value of 4.630302 in. above the datum compares very favorably to the Pro-E reported composite CG position of 4.630305 in. (additional decimal places are included only to illustrate the correctness of the approach).

The right side of the spreadsheet identifies the component height (or length, as applicable), the applicable thermal parameters, and the effects on the component's mass properties that result from the imposed temperature change. There are many miscellaneous components, such as screws, flat 
washers, and brackets that comprise the adapter ring. Given their small masses, the primary contribution they make to the changes in the mass properties of the entire adapter ring lies not in their individual dimensional changes rather, the translation they experience by virtue of the expansion or contraction of whatever they are attached to. This greatly simplifies the calculation with insignificant loss of accuracy. As indicated at the top of the spreadsheet expansion/contraction was only applied to components No. $1-5$. The remainder of the components moved with these, however they still contributed to a change in the overall CG position since their own CG positions relative to the adapter ring datum will have changed. Again, summing the moments and dividing by the total weight of the adapter ring yields the composite CG position relative to the datum. For the case shown, there was an average temperature of about $93.8 \mathrm{~F}^{\circ}$ in the adapter ring during the PART measurement, as shown in temperature map K-2, Appendix D. During the TARE measurement, this had dropped to an average of about $82.5 \mathrm{~F}^{\circ}$, as shown in temperature map K-6, Appendix D.

The PART conditions create a $23.8 \mathrm{~F}^{\mathrm{o}}\left(93.8^{\circ}-70.0^{\circ} \mathrm{F}\right)$ temperature increase over the assumed ambient temperature as shown in the adapter ring section of spreadsheet E-1. This change shifts the adapter ring CG to 4.631643 in. from its datum (the bottom of the feet of the adapter ring). This is a change of only $0.00134 \mathrm{in}$. from the room temperature CG position.

For the TARE conditions, the adapter ring temperatures were approximately $12.5^{\circ} \mathrm{F}\left(82.5^{\circ}-70.0^{\circ} \mathrm{F}\right)$ above room temperature. As shown in the adapter ring section of spreadsheet E-2, this shifts the adapter ring CG to $4.631007 \mathrm{in}$. from its datum, a change of only $0.00070 \mathrm{in}$. from the room temperature position. Therefore, the net effect in changing the fixture temperatures from those during the PART measurement to those applicable to the TARE measurement is to change the adapter ring $\mathrm{CG}$ distance from its datum by

$$
\Delta \mathrm{Y}_{0}{ }^{\prime}-\Delta \mathrm{Y}_{0}=4.631007 \text { in. }-4.631643 \text { in. }=-0.00064 \text { in. }
$$

The negative sign indicates a reduction in the distance from the datum during the TARE as compared to that during the PART measurement, as a result of the cooling that occurred.

\section{2. $\quad$ Rotation Fixture}

In principle, the rotation fixture was handled similarly to the adapter ring. However, the Pro-E model of this item was not as conducive to the type of detailed treatment made for the adapter ring. Furthermore, the only temperatures recorded in the temperature maps in Appendix D were below the pivot. So a few liberties were taken that yield conservative results, i.e., greater changes to the mass properties than likely occurred.

First, the upper structure of the rotation fixture above the pivot was presumed to be unaffected by thermal changes. Since the intent of these calculations is to evaluate the corrections to the MMRTG CG on its long axis, only the changes that occurred while in Position No. 2 are of interest. The temperature maps in Appendix D indicate that temperature changes in the rotation fixture diminished from the base plate toward the pivot and this trend no doubt continues above the pivot. Secondly, the temperature induced expansion or contraction that does occur in the upper members mimics what occurs in the lower structure, i.e., these structures all either expand or contract together, albeit not necessarily by the same amount nor at the same rate. There is a tendency for these to negate any overall translation of the rotation fixture's CG. By ignoring the changes that occur in the upper members, the calculated correction to the CG will be somewhat greater than the actual case. The overall uncertainty in the MMRTG CG includes a contribution from the temperature effects as quantitatively estimated by the results from this appendix. Overestimating this contribution will, therefore, be conservative i.e., introduce a slightly larger uncertainty band on the CG location. 
The rotation fixture was arbitrarily divided into five discrete sections for this analysis. These are listed in the rotation fixture section of spreadsheet E-2 (Table 8). The "plumb stanchions" are the four outboard members that appear to be plumb when viewed along the $\mathrm{Y}$ axis of the rotation fixture. The four "oblique braces" are the short inboard braces of the stanchions. The plumb stanchions were divided into two discrete sections: (1) that portion between the pivot and the attachment point of the oblique member and (2) that portion between the attachment point of the oblique member and the foot pad. The foot pads and the base plate were the other components considered. The masses of multiple items, such as the eight foot pads or the four upper stanchion sections, whose moment arms were identical, were lumped together. The apparatus around the pivot point was ignored, justified by their short moment arms from the pivot. Given the actual geometries of the stanchions and braces, the values assigned for the mass and CG location of each was necessarily crude. The total mass for these components summed to $110.6021 \mathrm{lb}$. This compared reasonably well to the Pro-E computed mass of $108.88 \mathrm{lb}$. In the interest of time, no attempt was made to refine the components' dimensions to obtain an improved match.

Computation of the new CG position was performed as described for the adapter ring. As an example, during the PART measurement the temperatures were approximately $10^{\circ} \mathrm{F}$ above the room temperatures (see temperature map K-2) and about the same during the TARE (temperature map K-6). Thus for the conditions during the MMRTG testing, no noticeable CG position change occurred in the rotation fixture between the PART and TARE measurements.

\section{Adjustment to the MMRTG CG Position}

From the development at the beginning of this appendix, the final correction to the MMRTG CG position can be found by combining the moment change from the adapter ring (AR) and that from the rotation fixture $(\mathrm{RF})$ :

$$
\frac{1}{W_{T A}} \sum_{j} W_{j} *\left(\Delta Y_{0}^{\prime}-\Delta Y_{0}\right)_{j}=\frac{1}{W_{T A}}\left[\sum_{A R} W_{j} *\left(\Delta Y_{0}^{\prime}-\Delta Y_{0}\right)_{j}+\sum_{R F} W_{j} *\left(\Delta Y_{0}^{\prime}-\Delta Y_{0}\right)_{j}\right]
$$

As shown before, the rotation fixture summation is zero, therefore the correction is from the adapter ring, - 0.00064 in., negative because the movement of the adapter ring CG is toward its mounting feet. Since the rotation fixture itself did not move, the adapter ring movement is away from the pivot. The conditions that occurred in the present case produced this very small displacement with obviously negligible significance. However, the development may be useful in future testing where much larger temperature changes occur in the fixtures. 


\section{Appendix F}

\section{Geometry Calculations}




\section{Appendix F Geometry Calculations}

\begin{tabular}{|c|c|c|c|}
\hline Parameter \& Its Origin & Description & Value & Units/Notes \\
\hline \multicolumn{4}{|l|}{ Per Design Drawings } \\
\hline $\mathrm{H}$ & & 31.980 & in \\
\hline$P_{L}$ & & 3.405 & in \\
\hline Pu & & 1.884 & in \\
\hline$L_{c}$ & Rotation-axis distance from MP datum, per Figure 26 & 26.000 & in \\
\hline$L_{R}$ & Rotation fixture pivot to upper base distance & 18.100 & in \\
\hline$L_{A R}$ & Height of adapter ring to top of mounting slides & 7.004 & in \\
\hline \multicolumn{4}{|l|}{ Derived Quantities } \\
\hline $\mathrm{Z}_{0}$ & $L_{C}-L_{R}+L_{A R}$ (Test-article datum distance from MP instr datum) & 14.904 & Test-article datum position constant \\
\hline$P_{L}+H+P_{U}$ & & 37.2690 & in \\
\hline $\mathrm{H}+\mathrm{Pu}_{U}$ & & 33.8640 & in \\
\hline \multicolumn{4}{|c|}{ Theoretical CG Position - Per Pro-E Analysis Relative to Test Article Coordinates (adjusted to X-Y in plane of base plate) } \\
\hline X"cG - (theoretical) & Confirms symmetry & 0.000 & in \\
\hline Y"cG - (theoretical) & Confirms symmetry & 0.000 & in \\
\hline$Z^{\prime \prime}$ CG - (theoretical) & Pro-E combined with fixture drawings (Figure 26) & 11.230 & in \\
\hline \multicolumn{4}{|c|}{ Best Estimates Averaged From Witnessed Metrology Determination [Relative to MP Instrument Coordinate System] } \\
\hline$x_{L}$ & & -0.003000 & in \\
\hline$x_{u}$ & & 0.000000 & in \\
\hline$Y_{L}$ & & 0.002610 & in \\
\hline$Y_{u}$ & & 0.004200 & in \\
\hline \multicolumn{4}{|c|}{ Geometric Calculations based on Metrological Measurements } \\
\hline$x_{0}$ & $X_{L}+\left(X_{U}-X_{L}\right)^{*} P_{L} /\left(P_{L}+H+P_{U}\right)$ & $-2.7259 \mathrm{E}-03$ & in, rounds to -0.003 in SE software \\
\hline$Y_{0}$ & $Y_{L}+\left(Y_{U}-Y_{L}\right)^{*} P_{L} /\left(P_{L}+H+P_{U}\right)$ & 2.7553E-03 & in, rounds to +0.003 in SE software \\
\hline$x_{U}-x_{L}$ & & $3.0000 \mathrm{E}-03$ & in \\
\hline$x_{U}-x_{0}$ & & $2.7259 \mathrm{E}-03$ & in \\
\hline$X_{0}-X_{L}$ & & 2.7409E-04 & in \\
\hline$Y_{U}-Y_{L}$ & & $1.5900 \mathrm{E}-03$ & in \\
\hline$Y_{u}-Y_{0}$ & & 1.4447E-03 & in \\
\hline$Y_{0}-Y_{L}$ & & 1.4527E-04 & in \\
\hline
\end{tabular}


Parameter \& Its Origin

$\mathrm{Z}_{\mathrm{CG} \text {-(measured) }}$

Ref: Run No. 1551

Description

Value

Units/Notes

Ref: Run No. 1586

Ref: Run No. 1602

Best Estimate (arithmetic average)

Top of Adapter Ring + Arithmetic Average of Runs

$X_{C G}^{\prime}=X_{C G}-X_{0}$

Adjusted for non-rounded $X_{0}$ value

$Y_{C G}^{\prime}=Y_{C G}-Y_{0}$

Adjusted for non-rounded $Y_{0}$ value

$Z_{C G}^{\prime}=Z_{C G}-Z_{0}$

Adjusted for non-rounded $Z_{0}$ value

Calculations Based on Test Rresults \& Geometric Data (see Table 2)

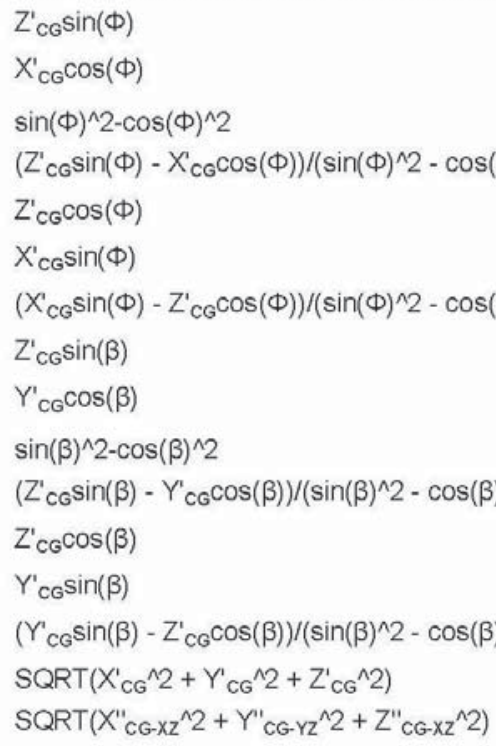

$\mathrm{R}^{\prime \prime C G}$ 


\section{Appendix G}

\section{Metrology Data Sheets and Data Reduction}




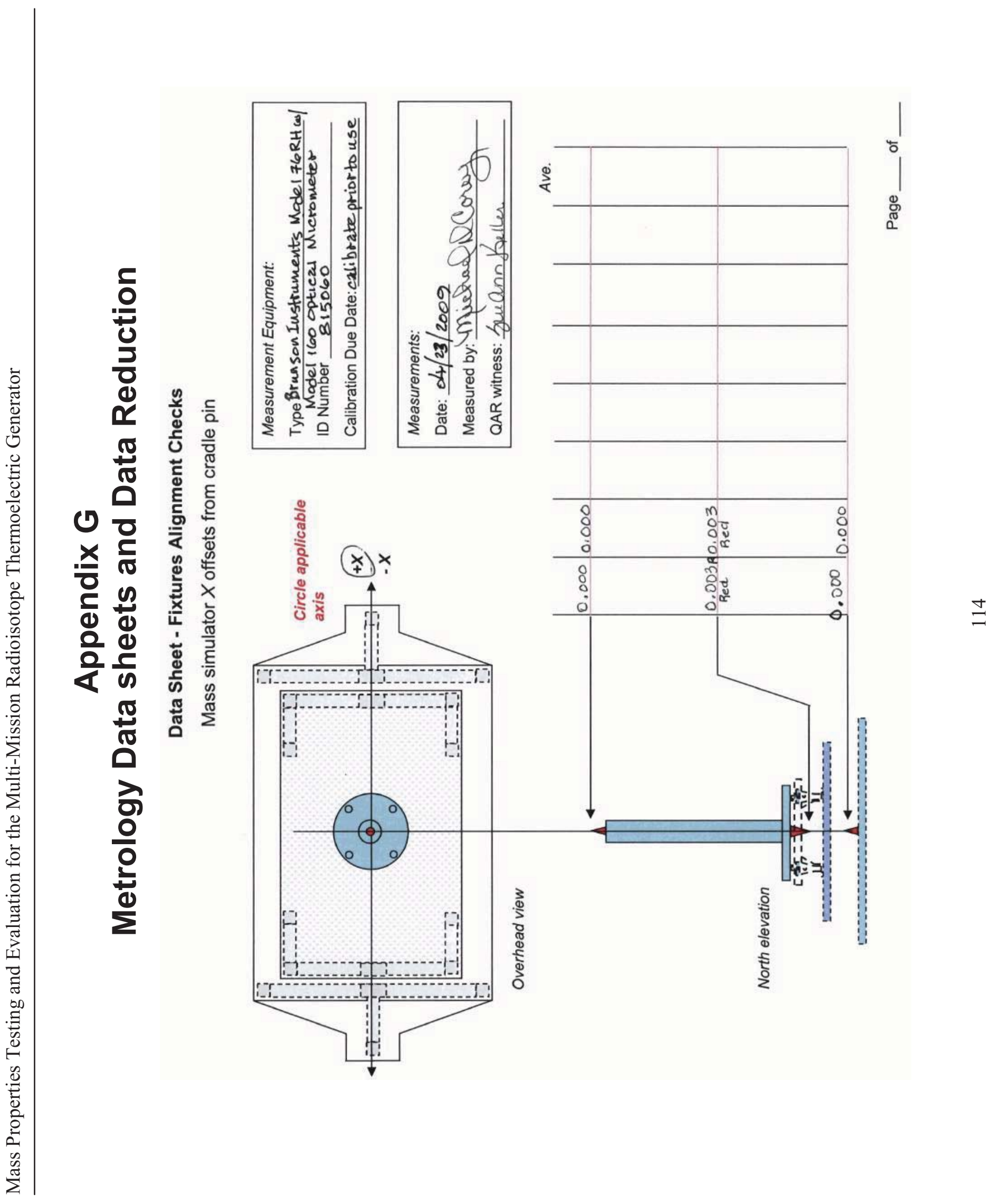




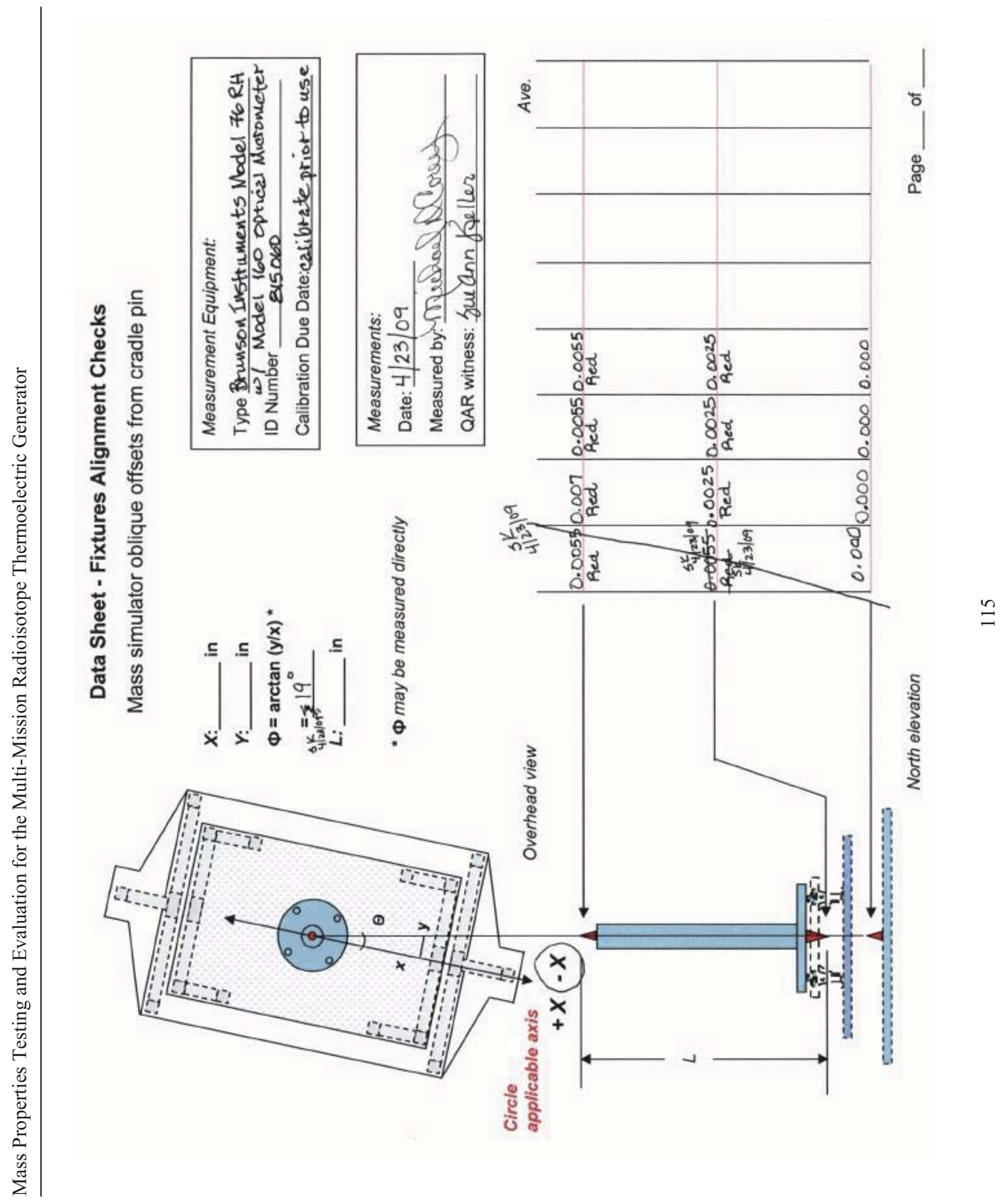




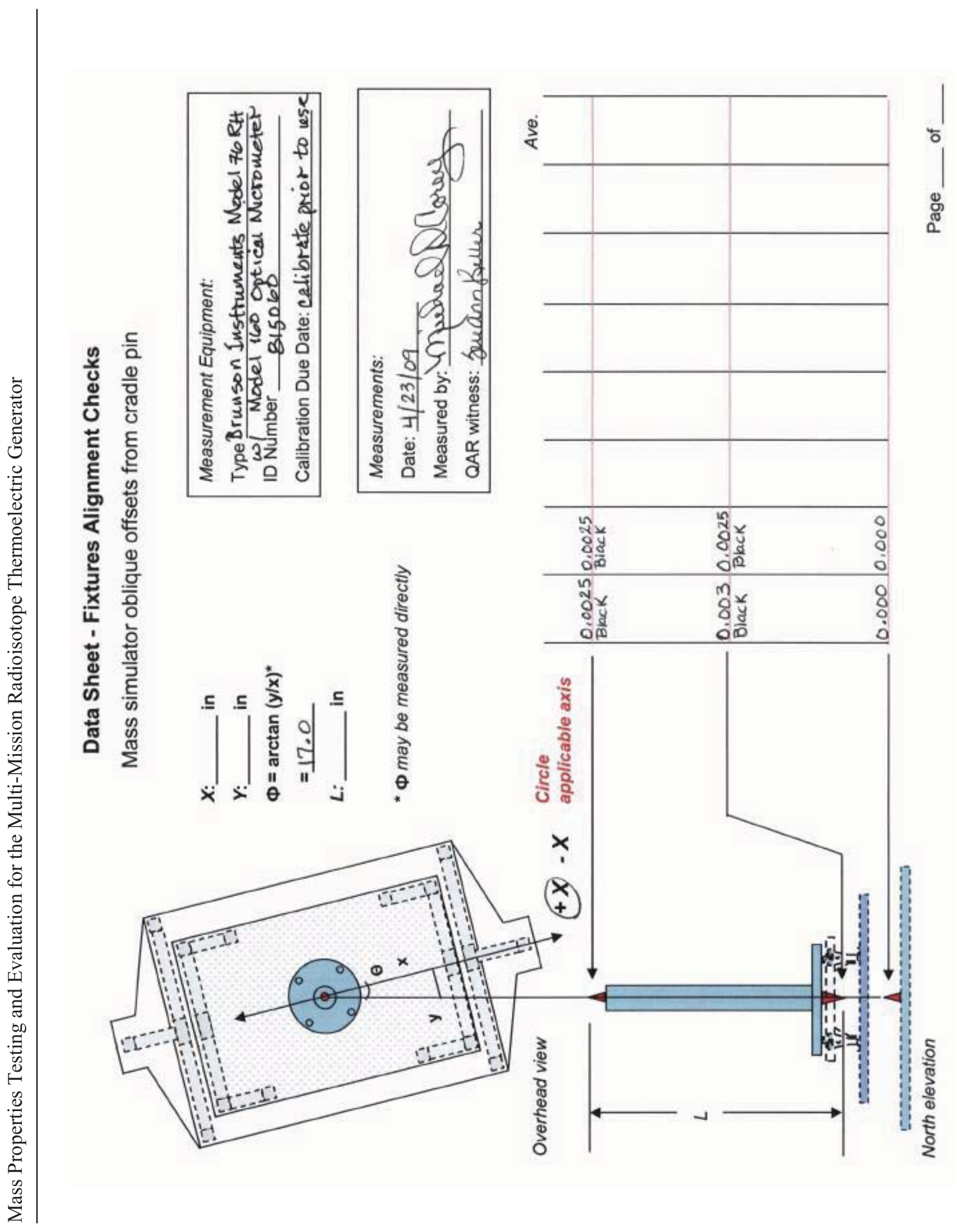




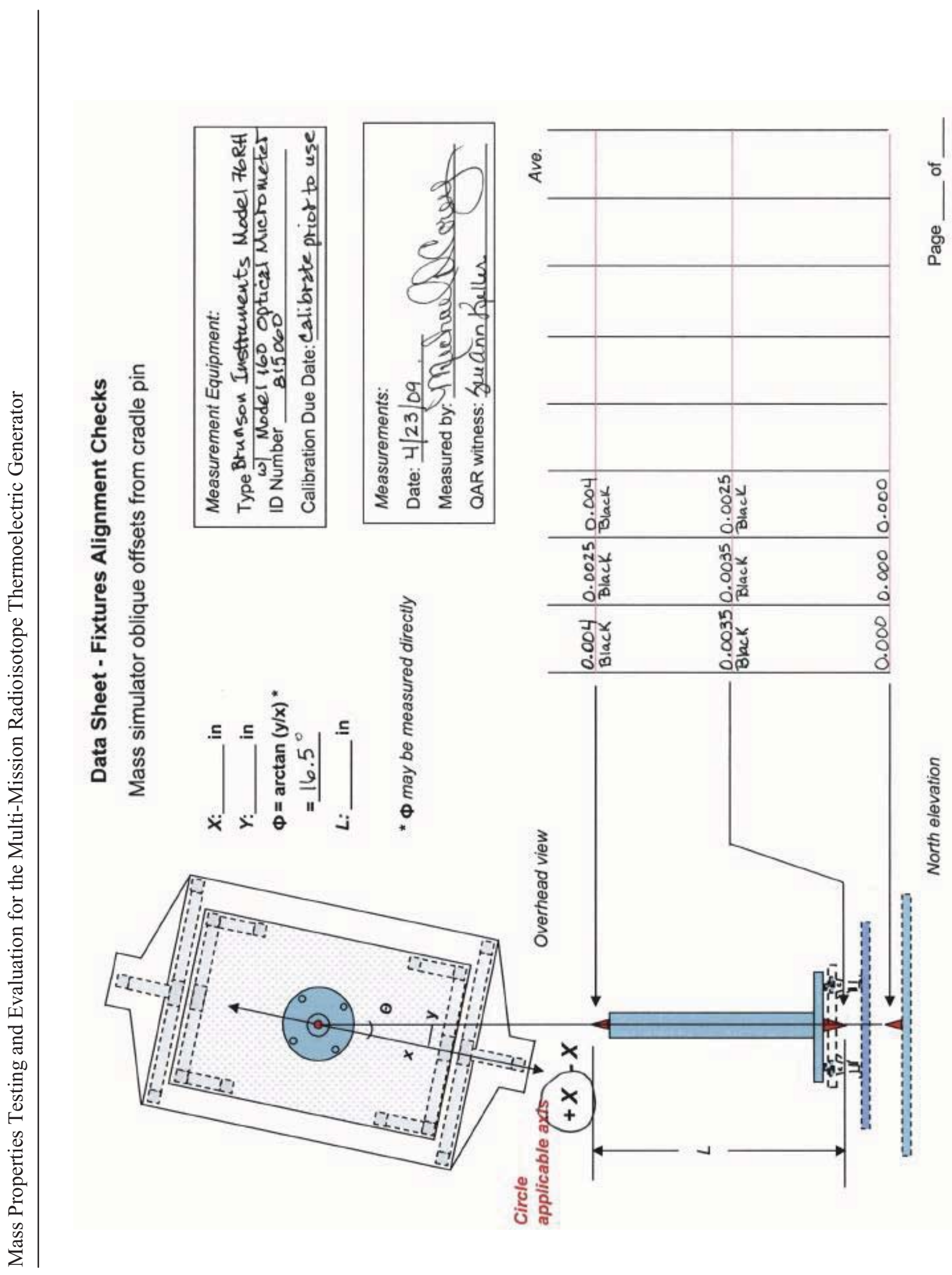

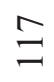




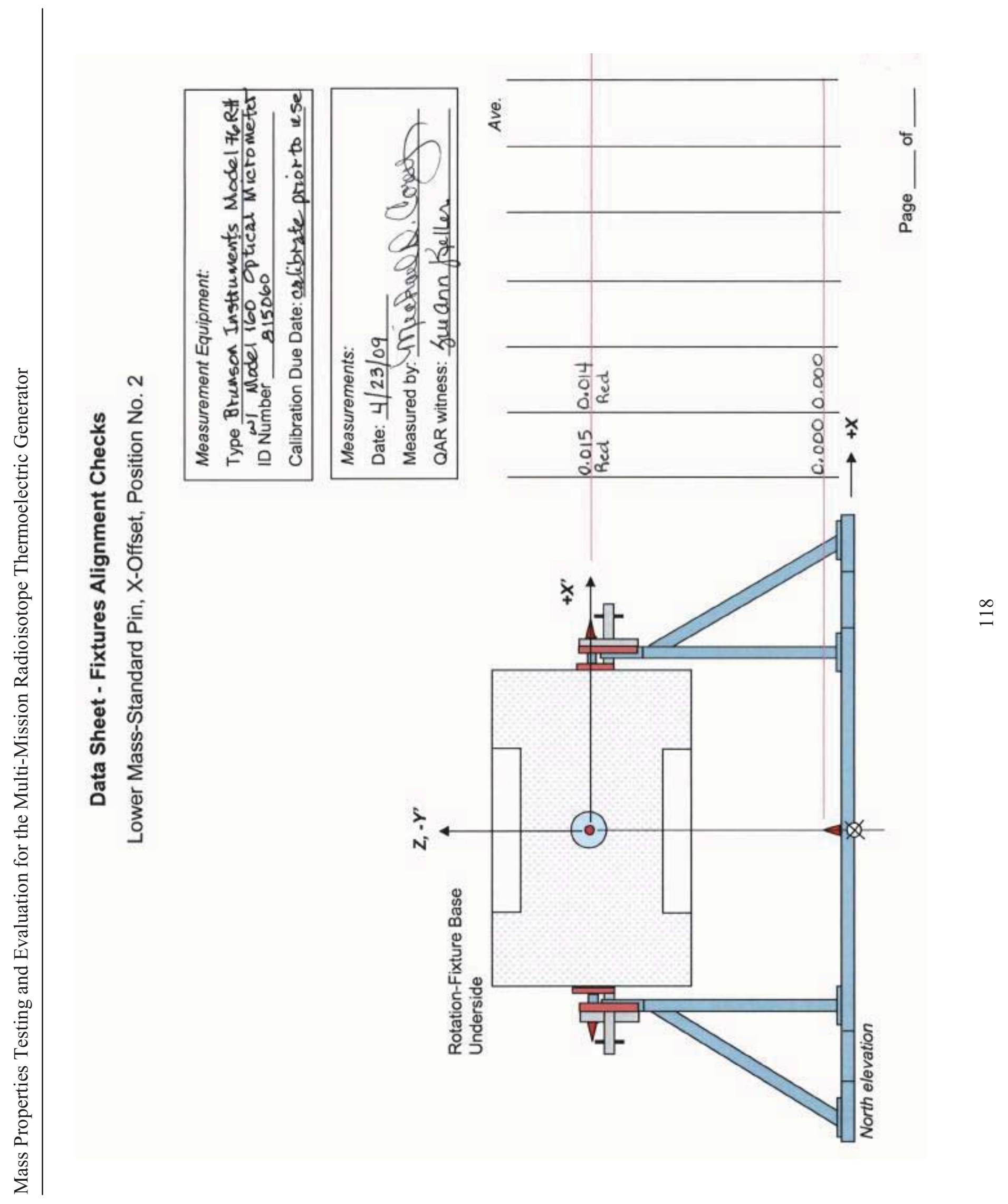




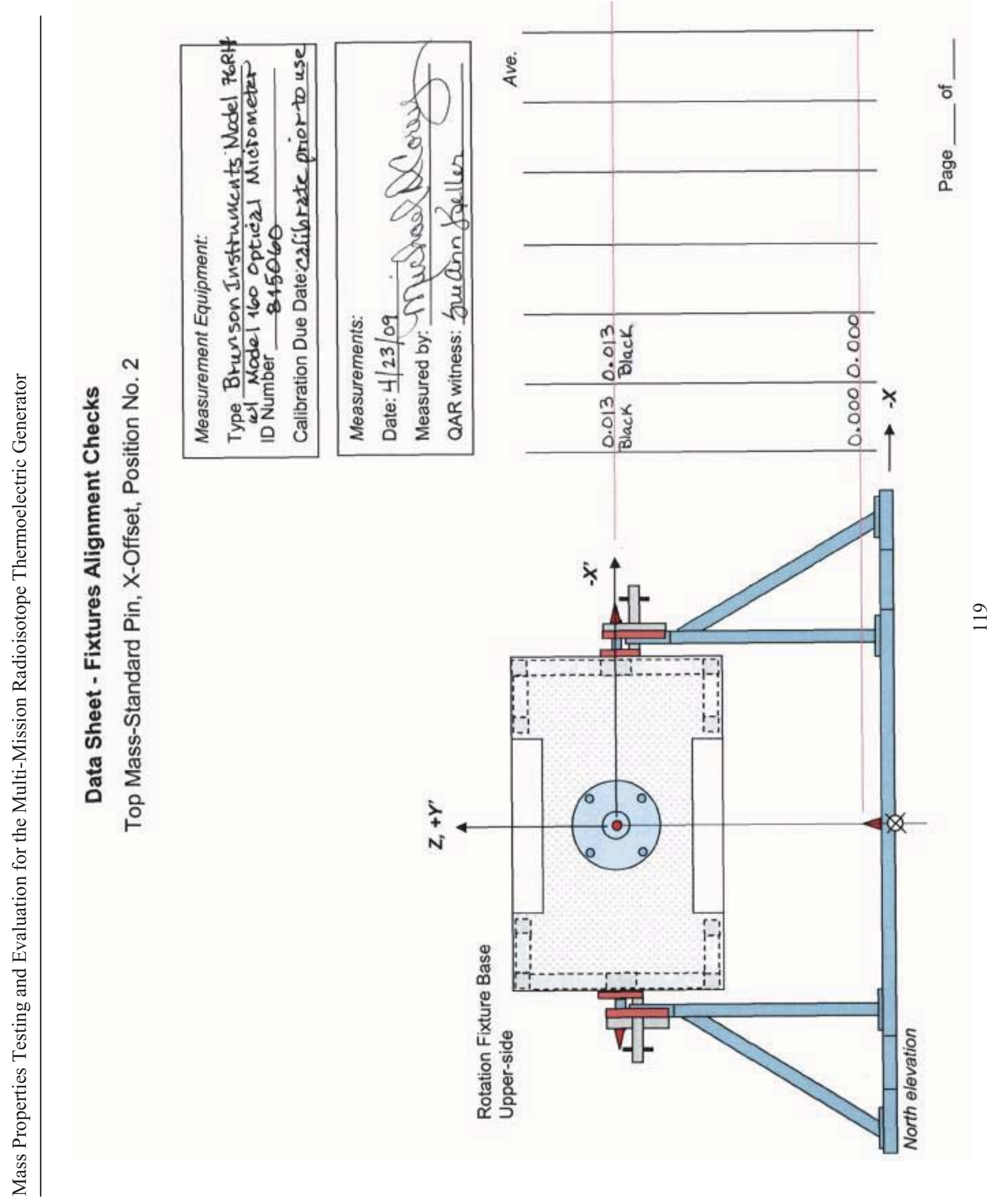




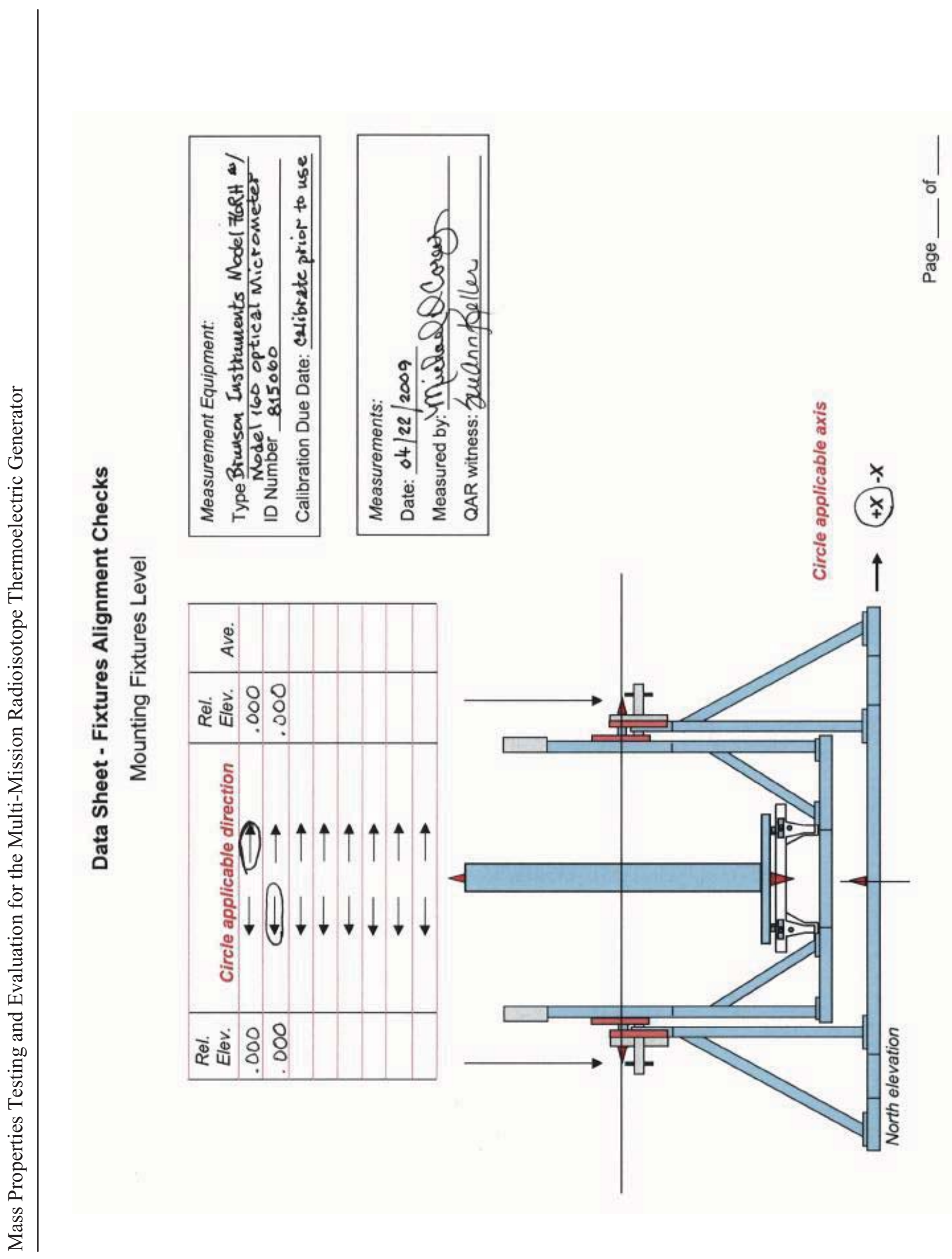




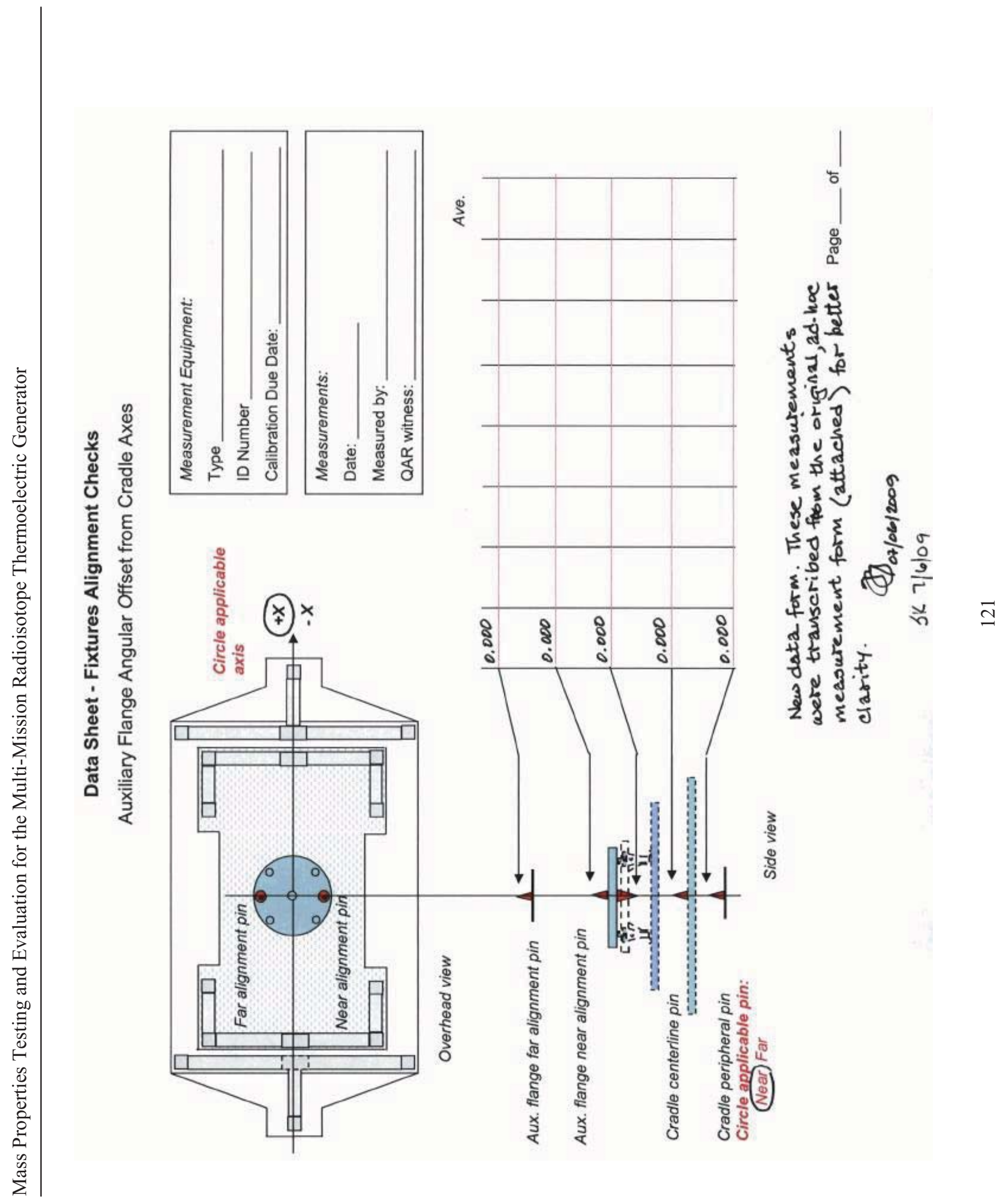




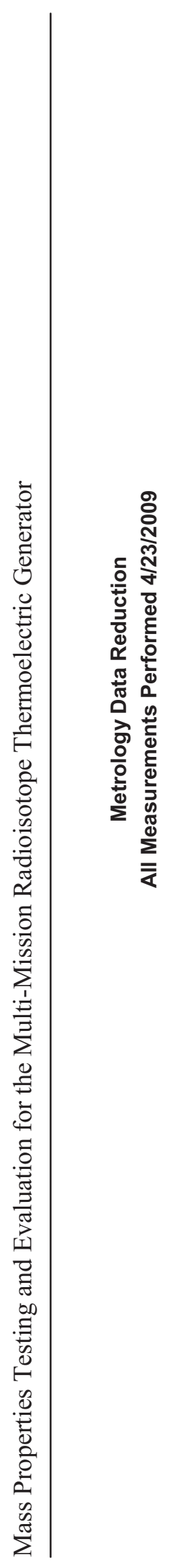

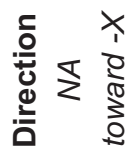

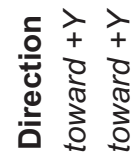

¿

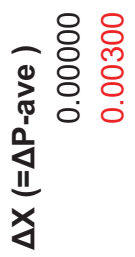

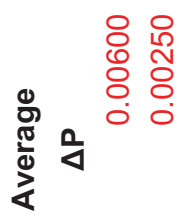

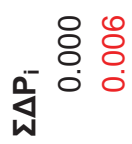

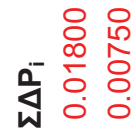

$z \sim N$

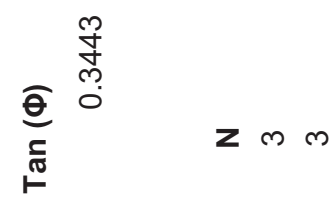

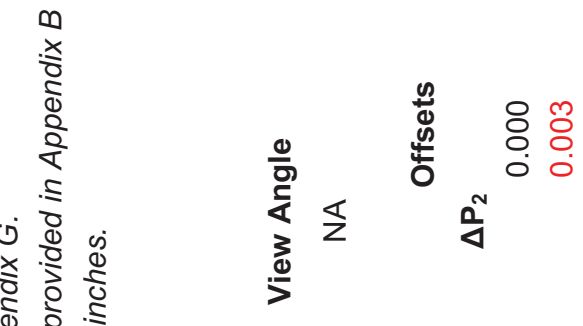
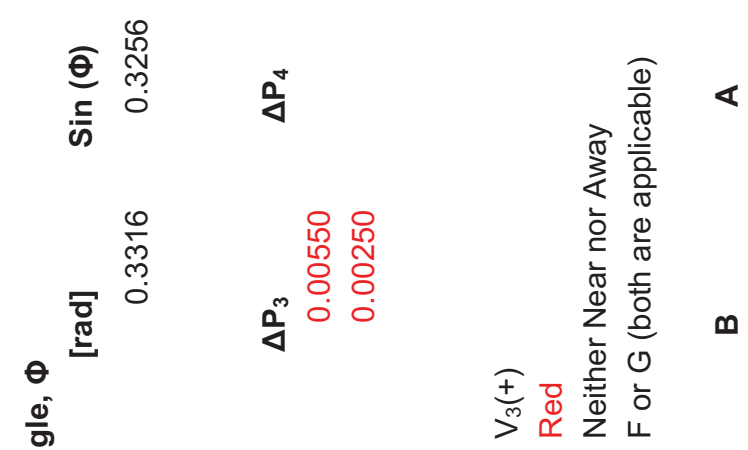

ปี

$\frac{8}{2}:$

बरे के ब

की

क $\frac{1}{5}$

苑

0.

₹ ह ई

is

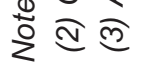

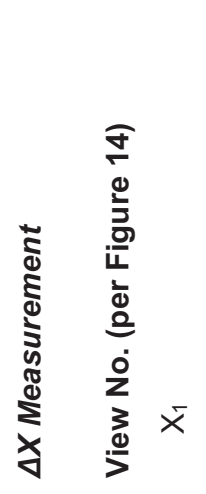

웅 :

$\theta$
$\frac{0}{0}$
$\frac{1}{4}$
3
3

음

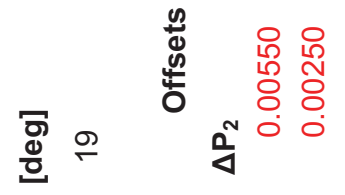

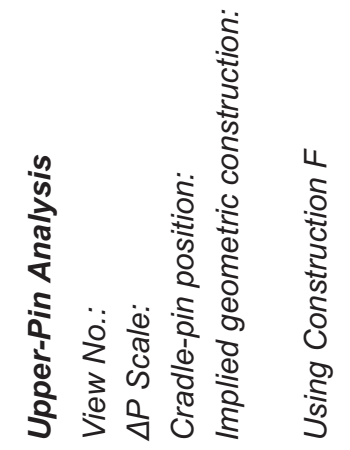

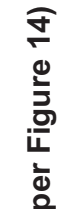

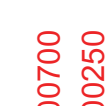

40

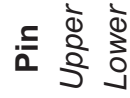




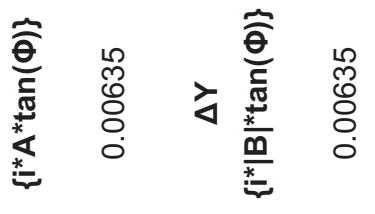

毫高

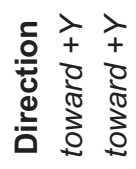

- -

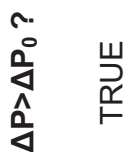

논

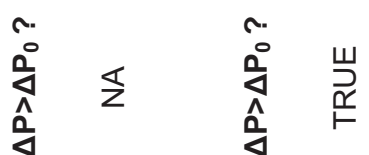

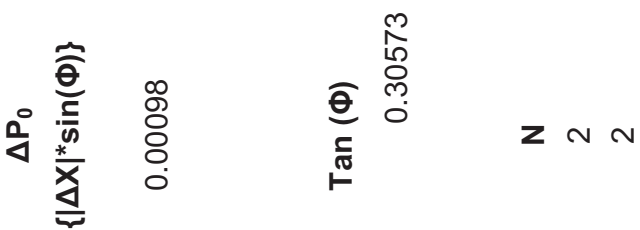

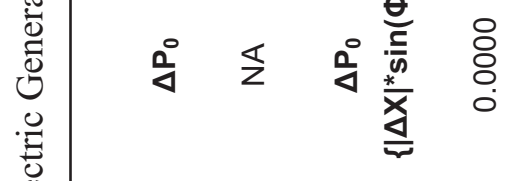

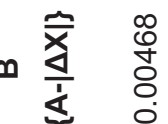

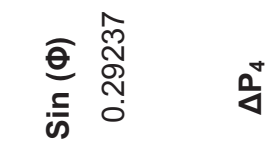

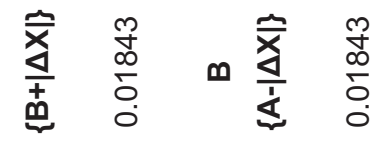

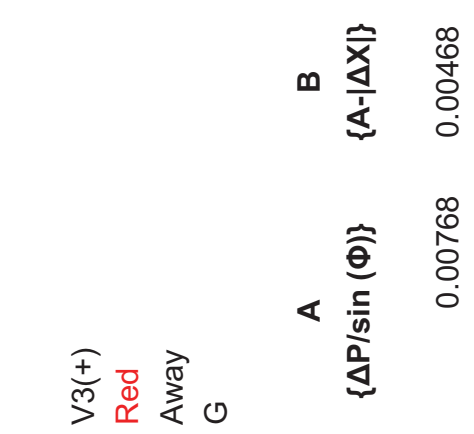

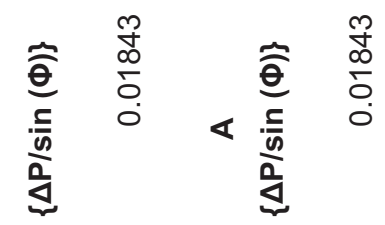
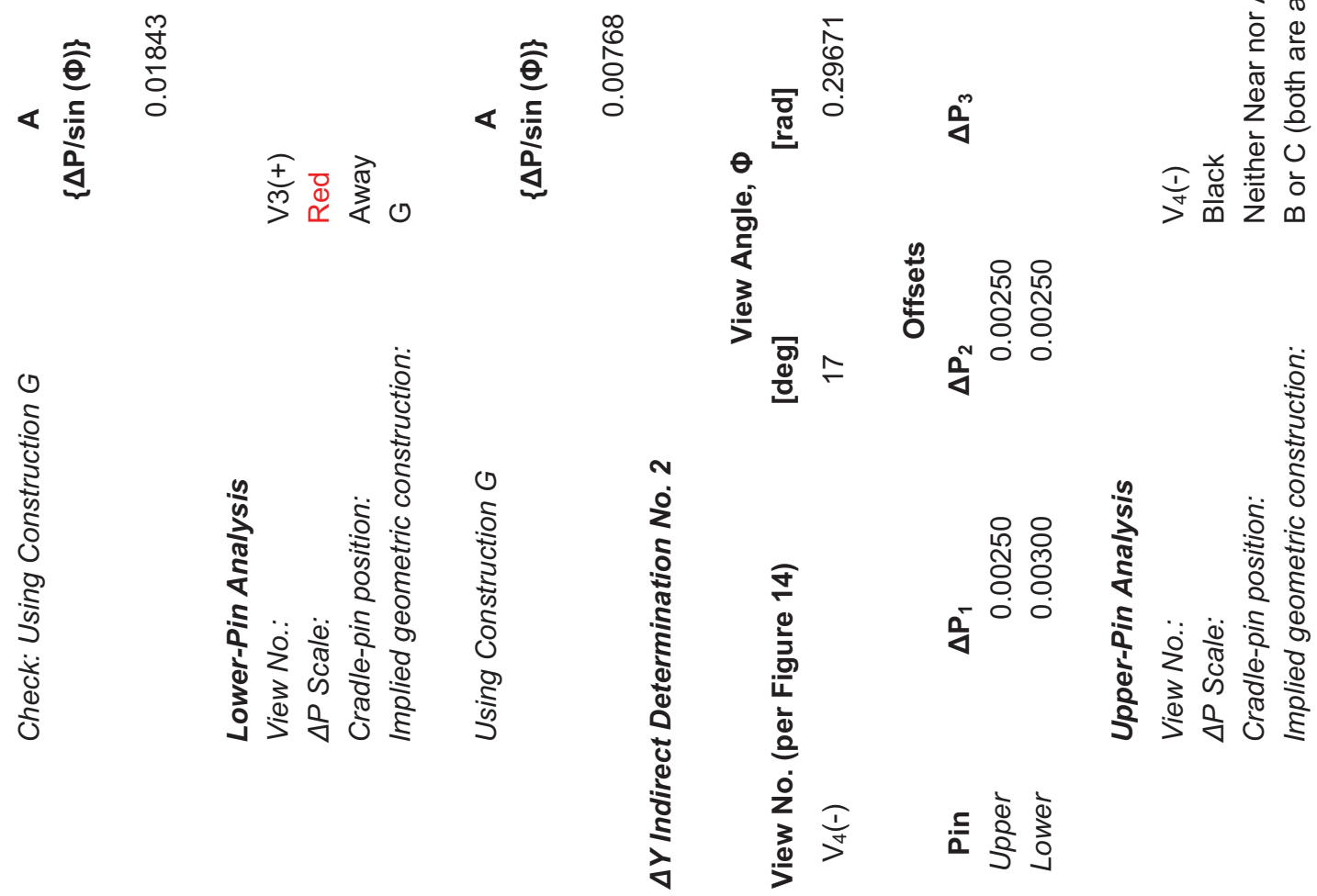


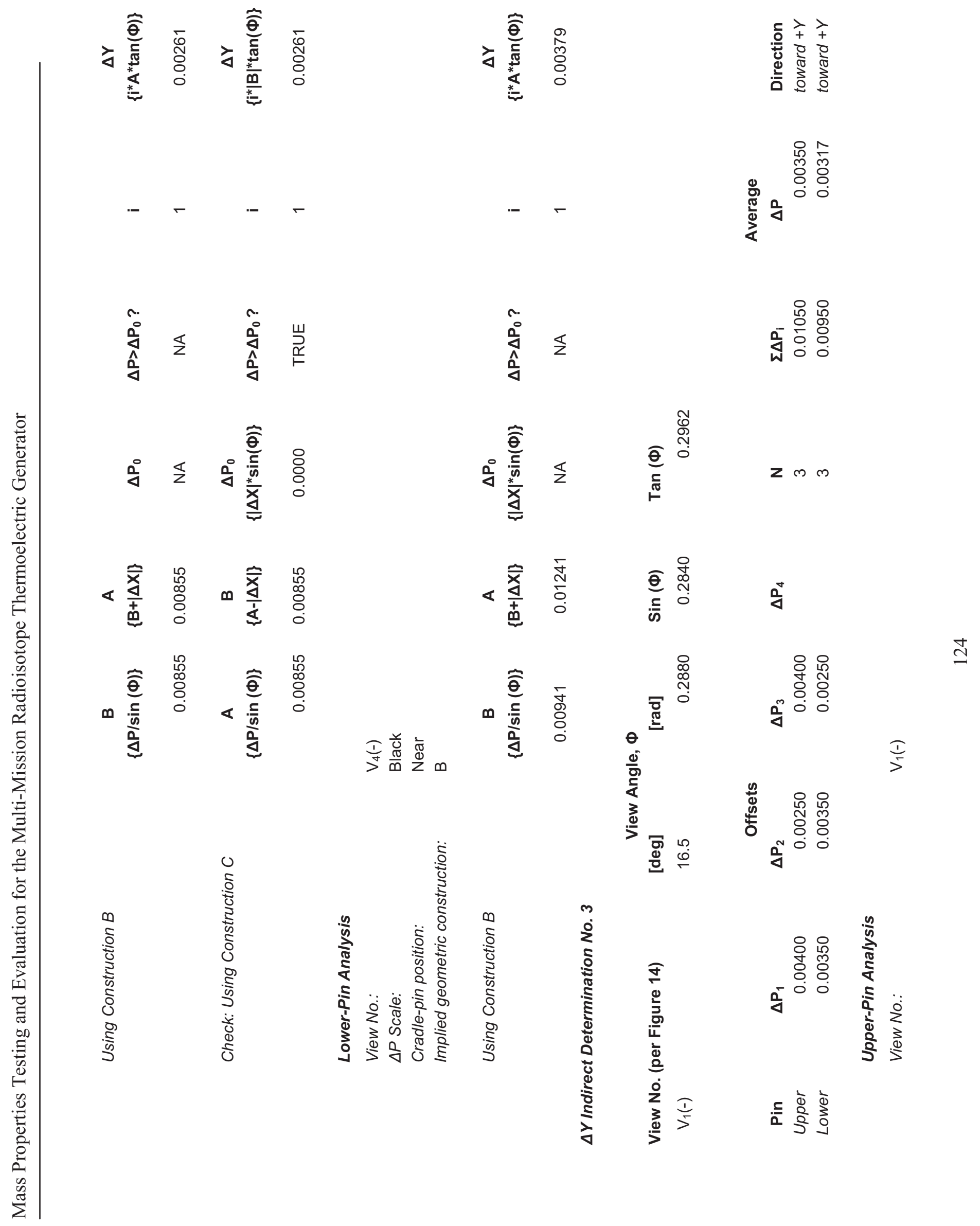




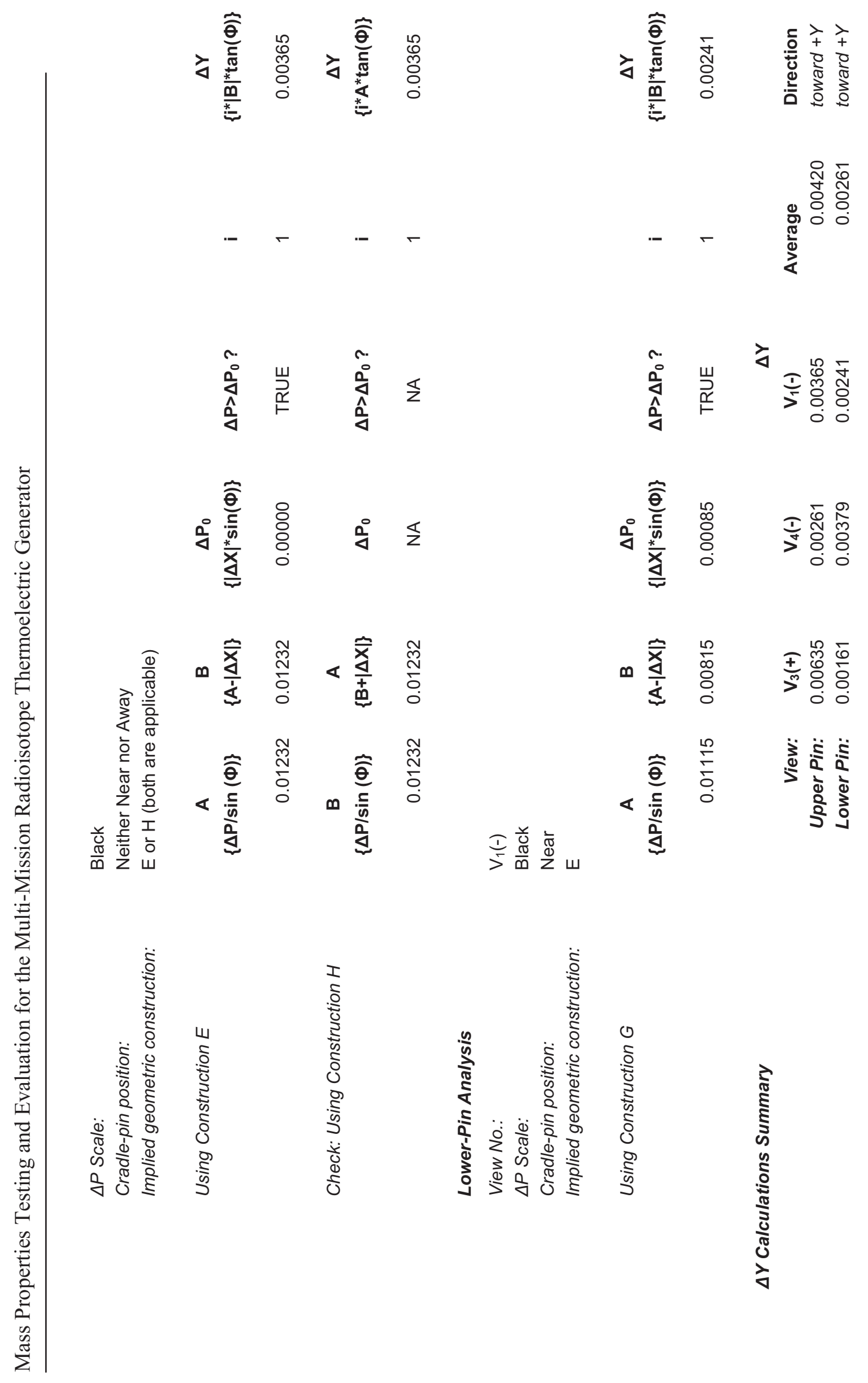


INTENTIONALLY BLANK 


\section{Appendix $\mathrm{H}$}

\section{Data Sheet - Weigh Scale Calibration Check}




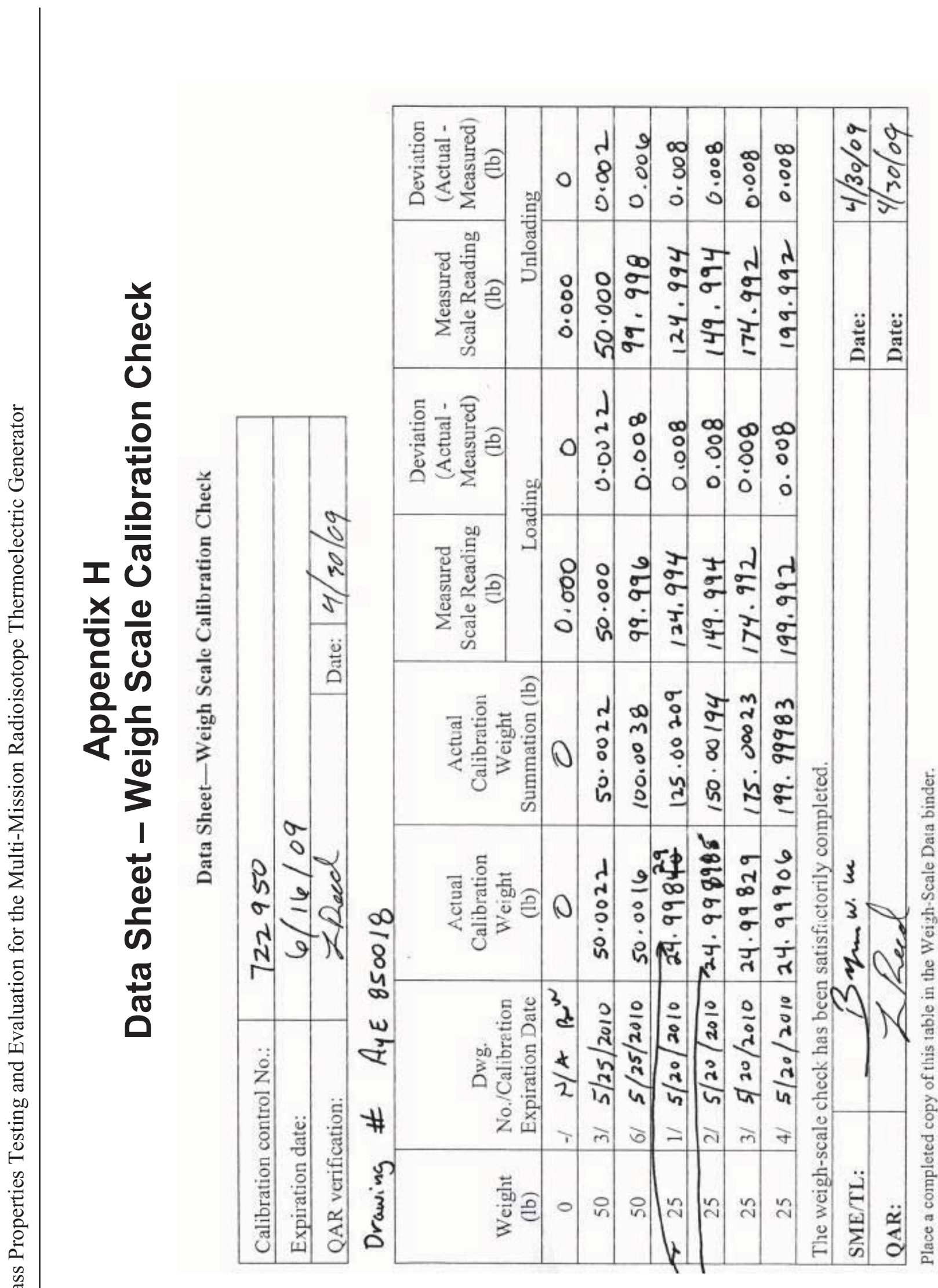


Appendix I

\section{Measurement System Output - MMRTG}




\section{Appendix I Measurement System Output - MMRTG}

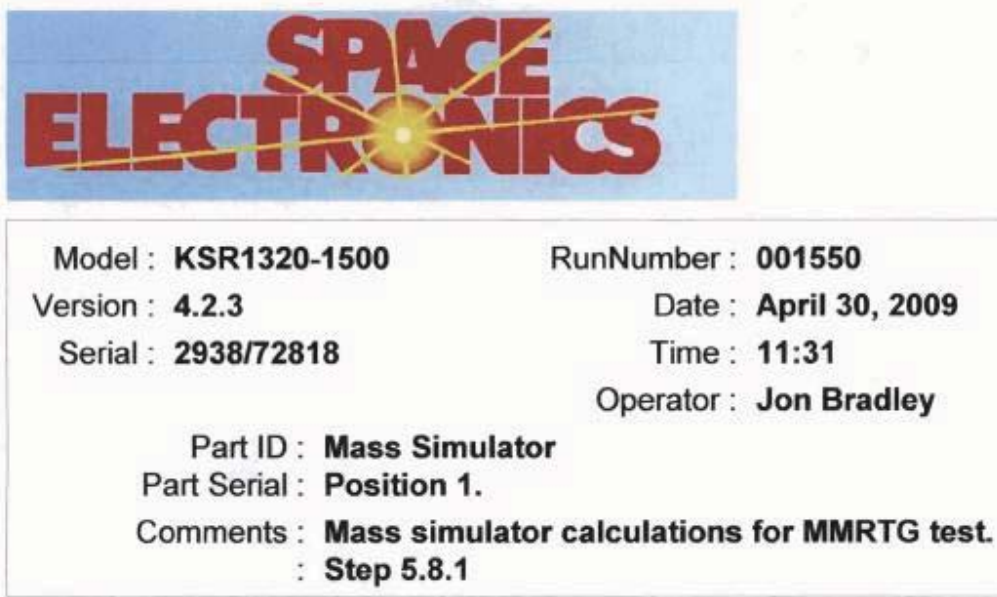

Model : KSR1320-1500

Version : 4.2 .3

Serial : $2938 / 72818$

\author{
RunNumber : 001550 \\ Date : April 30, 2009 \\ Time: 11:31 \\ Operator : Jon Bradley
}

Part ID : Mass Simulator

Part Serial: Position 1.

Comments : Mass simulator calculations for MMRTG test.

Step 5.8.1

\section{Calculation Data}

CG Tare : 001544

CG Part : 001538

April 30, 2009

April 29, 2009

Part Weight :

97.1980

lb

09:29

$16: 33$

Fixture Tare after simulator removed Mass Simulator

Part Estimated CG Height : $\quad \mathbf{2 6 . 1 0 0 0}$ in

Machine Angle at Part 0 Degrees : 0.000 deg

User Angle System Polarity : Positive (CCW INCREASING ANGLE)

User Datums Offset below are in Machine Angle System
User Datum Offset X :
-0.0030 in
User Datum Offset $Y$ :
$\mathbf{0 . 0 0 3 0}$ in

\section{Calculation Results}

\section{Calculated Static CG (see Note 1)}

\begin{tabular}{|r|c|r|}
\hline $\begin{array}{l}\text { Machine } \\
\text { Referenced }\end{array}$ & $\begin{array}{c}\text { CG Offset } \\
\text { (in) }\end{array}$ & $\begin{array}{c}\text { CG Moment } \\
\text { (lb-in) }\end{array}$ \\
\hline Static CG X: & $\mathbf{0 . 0 0 0 2}$ & $\mathbf{0 . 0 1 5}$ \\
\hline Static CG Y: & $\mathbf{0 . 0 0 3 1}$ & $\mathbf{0 . 2 9 8}$ \\
\hline Static CG Mag : & $\mathbf{0 . 0 0 3 1}$ & $\mathbf{0 . 2 9 9}$ \\
\hline Static CG Angle : & $\mathbf{8 7 . 1 1 1}$ & $\mathbf{8 7 . 1 1 1}$ \\
\hline
\end{tabular}

\begin{tabular}{|r|r|r|}
\hline $\begin{array}{l}\text { User } \\
\text { Referenced }\end{array}$ & $\begin{array}{c}\text { CG Offset } \\
\text { (in) }\end{array}$ & $\begin{array}{c}\text { CG Moment } \\
\text { (lb-in) }\end{array}$ \\
\hline Static CG X: & 0.0032 & 0.307 \\
\hline Static CG Y : & 0.0001 & 0.007 \\
\hline Static CG Mag : & 0.0032 & 0.307 \\
\hline Static CG Angle : & 1.253 & 1.253 \\
\hline
\end{tabular}

Notes

1. CG Uncertainty

CG Moment Uncertainty

CG Offset Uncertainty

$0.169 \mathrm{lb}$-in

0.0017 in 


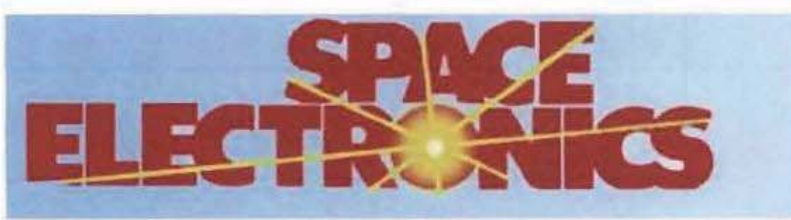

Model : KSR1320-1500

Version : 4.2 .3

Serial : $2938 / 72818$
RunNumber : 001627

Date : December 16, 2009

Time : 13:53

Operator : Frank Felicione

Part ID : Mass Simulator

Part Serial: Position 2.

Comments : Revised calculations with corrected Z-datum offset

Step 5.8.2

\section{Calculation Data}

CG Tare : 001547

CG Part : 001541

April 30, 2009

April 29, 2009

Part Weight :

97.1980

lb
$10: 19$

$17: 29$
Fixture Tare after simulator removed Mass Simulator

Part Estimated CG Height : $\mathbf{2 6 . 0 0 0 0}$ in

Machine Angle at Part 0 Degrees : $\quad 359.998$ deg

User Angle System Polarity : Positive (CCW INCREASING ANGLE)

User Datums Offset below are in Machine Angle System

User Datum Offset X : $\quad-\mathbf{0 . 0 1 4 0}$ in $\quad$ User Datum Offset Z: $\mathbf{- 1 1 . 0 9 6 0}$ in

\section{Calculation Results}

Calculated Static CG (see Note 1)

\section{Machine Referenced}

\begin{tabular}{|r|r|r|}
\hline Static CG X : & -0.0073 & -0.709 \\
\hline Static CG Y : & 0.1298 & 12.614 \\
\hline Static CG Mag : & 0.1300 & 12.634 \\
\hline Static CG Angle : & 93.216 & 93.216
\end{tabular}

\begin{tabular}{|r|r|r|}
\hline $\begin{array}{l}\text { User } \\
\text { Referenced }\end{array}$ & $\begin{array}{c}\text { CG Offset } \\
\text { (in) }\end{array}$ & $\begin{array}{c}\text { CG Moment } \\
\text { (lb-in) }\end{array}$ \\
\hline Static CG X : & $\mathbf{0 . 0 0 6 3}$ & $\mathbf{0 . 6 1 4}$ \\
\hline Static CG Z : & 11.2258 & 1091.123 \\
\hline Static CG Mag : & 11.2258 & $\mathbf{1 0 9 1 . 1 2 3}$ \\
\hline Static CG Angle : & 89.968 & 89.968 \\
\hline
\end{tabular}

Notes

1. CG Uncertainty

CG Moment Uncertainty

CG Offset Uncertainty 


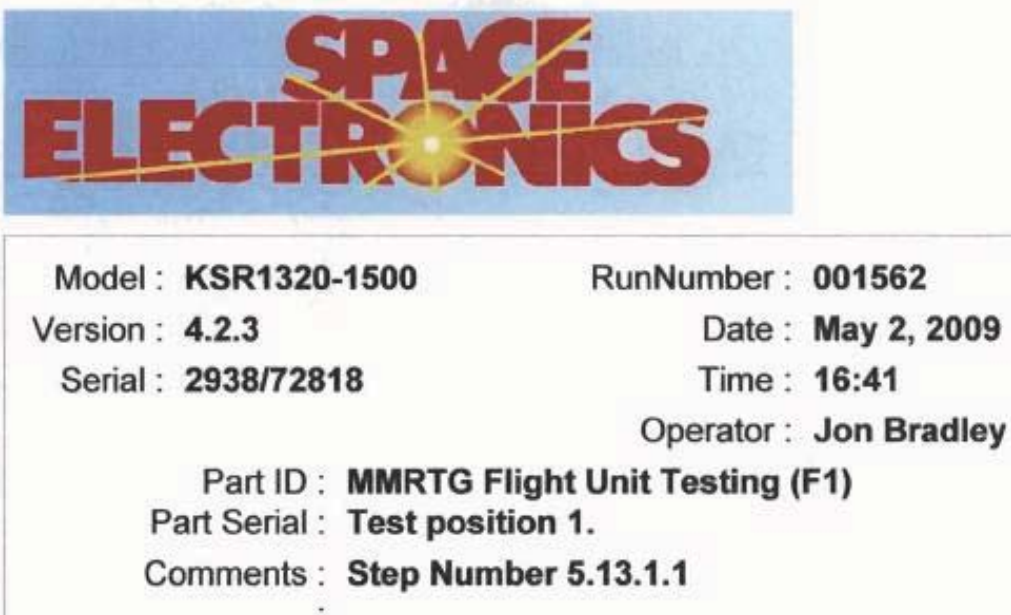

\section{Calculation Data}

CG Tare : 001560

May 2, 2009

11:57

Post Test Fixture Tare

CG Part : 001553 May 1, 2009

18:02

MMRTG Flight Unit Testing (F1)

Part Weight: $\quad \mathbf{1 0 0 . 1 1 7 0 ~ l b ~}$

Part Estimated CG Height : 26.1000 in

Machine Angle at Part 0 Degrees : $\quad 0.000$ deg

User Angle System Polarity : Positive (CCW INCREASING ANGLE)

User Datums Offset below are in Machine Angle System

User Datum Offset X : $\quad-\mathbf{0 . 0 0 3 0}$ in $\quad$ User Datum Offset $Y$ : $\quad \mathbf{0 . 0 0 3 0}$ in

\section{Calculation Results}

Calculated Static CG (see Note 1)

\begin{tabular}{|r|r|r|}
\hline $\begin{array}{l}\text { Machine } \\
\text { Referenced }\end{array}$ & $\begin{array}{c}\text { CG Offset } \\
\text { (in) }\end{array}$ & $\begin{array}{c}\text { CG Moment } \\
\text { (lb-in) }\end{array}$ \\
\hline Static CG X: & $\mathbf{0 . 0 9 3 8}$ & $\mathbf{9 . 3 8}$ \\
\hline Static CG Y : & $\mathbf{0 . 0 3 0 6}$ & $\mathbf{3 . 0 6}$ \\
\hline Static CG Mag : & $\mathbf{0 . 0 9 8 6}$ & $\mathbf{9 . 8 7}$ \\
\hline Static CG Angle : & $\mathbf{1 8 . 0 6 5}$ & $\mathbf{1 8 . 0 6 5}$ \\
\hline
\end{tabular}

\begin{tabular}{|r|r|r|}
\hline $\begin{array}{l}\text { User } \\
\text { Referenced }\end{array}$ & $\begin{array}{c}\text { CG Offset } \\
\text { (in) }\end{array}$ & $\begin{array}{c}\text { CG Moment } \\
\text { (lb-in) }\end{array}$ \\
\hline Static CG X: & 0.0968 & 9.687 \\
\hline Static CG Y : & 0.0276 & 2.762 \\
\hline Static CG Mag : & 0.1006 & 10.073 \\
\hline Static CG Angle : & 15.911 & 15.911 \\
\hline
\end{tabular}

Notes
1. CG Uncertainty
CG Moment Uncertainty
CG Offset Uncertainty




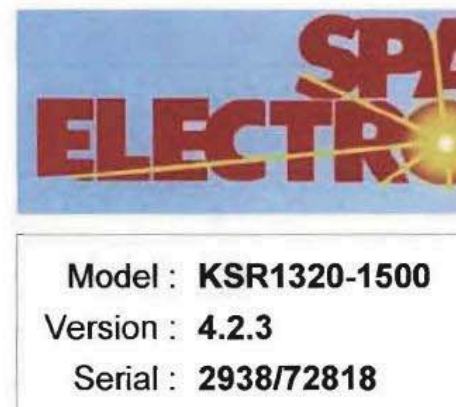

$$
\begin{aligned}
\text { RunNumber : } & 001626 \\
\text { Date : } & \text { December 16, } 2009 \\
\text { Time : } & 13: 43 \\
\text { Operator : } & \text { Frank Felicione }
\end{aligned}
$$

Part ID : MMRTG Flight Unit Testing (F1)

Part Serial : Position 2.

Comments : Revised calculations with corrected Z-datum offset Step 5.13.2.1

\section{Calculation Data}

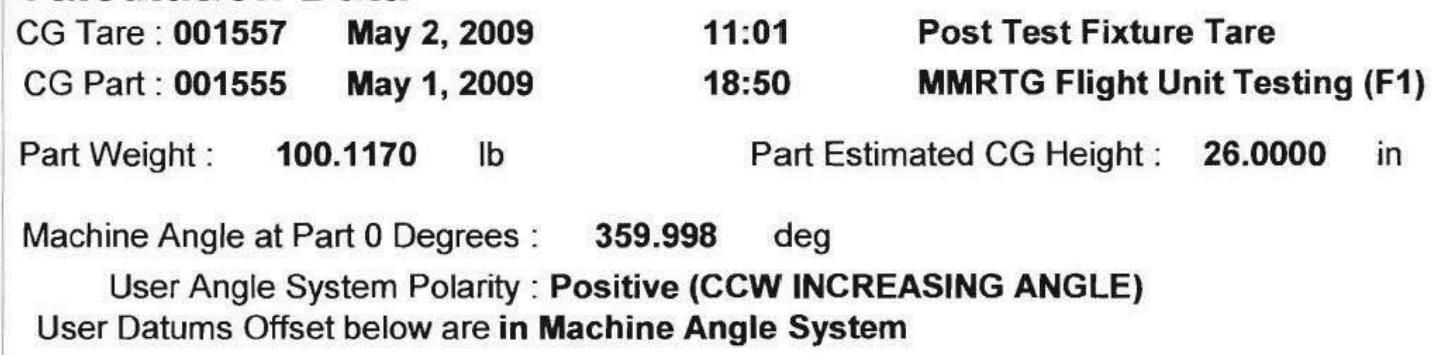

\section{Calculation Results}

Calculated Static CG (see Note 1)

\begin{tabular}{|r|r|r|}
\hline $\begin{array}{l}\text { Machine } \\
\text { Referenced }\end{array}$ & \multicolumn{1}{c|}{$\begin{array}{c}\text { CG Offset } \\
\text { (in) }\end{array}$} & $\begin{array}{c}\text { CG Moment } \\
\text { (lb-in) }\end{array}$ \\
\hline Static CG X : & $\mathbf{0 . 0 8 7 2}$ & $\mathbf{8 . 7 3 1}$ \\
\hline Static CG Y : & $-\mathbf{- 0 . 2 8 0 3}$ & $-\mathbf{2 8 . 0 5 9}$ \\
\hline Static CG Mag : & $\mathbf{0 . 2 9 3 5}$ & $\mathbf{2 9 . 3 8 6}$ \\
\hline Static CG Angle : & $\mathbf{2 8 7 . 2 8 4}$ & $\mathbf{2 8 7 . 2 8 4}$ \\
\hline
\end{tabular}

\begin{tabular}{|r|r|r|}
\hline $\begin{array}{l}\text { User } \\
\text { Referenced }\end{array}$ & \multicolumn{1}{|c|}{$\begin{array}{c}\text { CG Offset } \\
\text { (in) }\end{array}$} & $\begin{array}{c}\text { CG Moment } \\
\text { (lb-in) }\end{array}$ \\
\hline Static CG X : & $\mathbf{0 . 1 0 0 8}$ & $\mathbf{1 0 . 0 9 5}$ \\
\hline Static CG Z : & $\mathbf{1 0 . 8 1 5 7}$ & $\mathbf{1 0 8 2 . 8 3 9}$ \\
\hline Static CG Mag : & $\mathbf{1 0 . 8 1 6 2}$ & $\mathbf{1 0 8 2 . 8 8 6}$ \\
\hline Static CG Angle : & $\mathbf{8 9 . 4 6 6}$ & $\mathbf{8 9 . 4 6 6}$ \\
\hline
\end{tabular}

\begin{tabular}{|l|}
\hline Notes \\
1. CG Uncertainty \\
CG Moment Uncertainty \\
CG Offset Uncertainty \\
\end{tabular}


INTENTIONALLY BLANK 


\section{Appendix J}

\section{MMRTG Weighing Record}




\section{Appendix $\mathrm{J}$ MMRTG Weighing Record}

Note: The pages in this appendix were copied from the actual MMRTG test procedure, RPS/HS-OI-28220. The header information and appendix designator (D) shown on these pages pertain to the test procedure document, not to the present document.

Idaho National Laboratory
\begin{tabular}{|c|lll|}
\hline MMRTG MASS PROPERTIES TEST & $\begin{array}{l}\text { Identifier: } \\
\text { Revision: }\end{array}$ & 0 \\
& Effective Date: & $04 / 28 / 09$ & Page: 129 of 167 \\
\hline
\end{tabular}

Appendix D

Data Sheet - Equipment and Fixtures Attached to MMRTG During Weighing or Testing

1. Measured MMRTG gross weight (Subsection 5.9.3.3): $\quad 162.776 \mathrm{lb}[\mathrm{N} 1]$.

2. Ancillary items present during MMRTG weighing but removed during testing:

\begin{tabular}{|c|c|c|c|}
\hline \multicolumn{4}{|c|}{ Item } \\
\hline \multicolumn{4}{|c|}{ Hoist plate (with swivel ring and (3) attachment pins and lanyards) } \\
\hline \multicolumn{4}{|c|}{ Lifting ring (with (3) attachment pins and lanyards) } \\
\hline \multicolumn{4}{|c|}{ Connector saver cable (without shorting plug) } \\
\hline \multicolumn{4}{|c|}{ Lockwire (if applicable) $\quad N / A$ Bw } \\
\hline \multicolumn{4}{|c|}{$\begin{array}{l}\text { Mounting screws, washers, and locking nuts used to attach the MMRTG to the PWR (stainless } \\
\text { steel) adapter ring }\end{array}$} \\
\hline \multicolumn{4}{|c|}{ PWR (stainless steel) adapter ring assembly } \\
\hline \multicolumn{4}{|c|}{ Cable or Varglas ties (if applicable) } \\
\hline a. & $\begin{array}{l}\text { Aggregate weight of ancillary items listed above } \\
\text { (Subsection 5.9.8): }\end{array}$ & 63.664 & $\mathrm{lb}[\mathrm{N} 2]$ \\
\hline b. & $\begin{array}{l}\text { Weight of the shorting plug present during weighing (not } \\
\text { necessarily the same one used for testing): } \\
\text { S/N } 28680100902\end{array}$ & 0.1942 & $\mathrm{lb}[\mathrm{N} 3]$ \\
\hline c. & $\begin{array}{l}\text { Weight of all ancillary items present during weighing but } \\
\text { absent during testing [N2] }+[\mathrm{N} 3] \text { : }\end{array}$ & 63.858 & $\mathrm{lb}[\mathrm{N} 4]$ \\
\hline
\end{tabular}




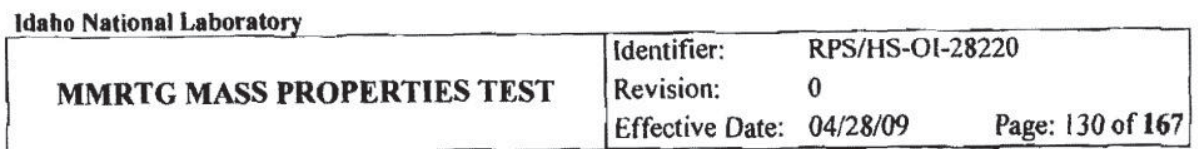

4. Additional hardware attached during testing but not present when weighed-CCY-cable, bracket, fasteners, connector caps, and shorting plug (all items in their physically correct positions). The information in this section is supplied by the Design Agency.

a. Serial numbers/or ID part numbers:

$$
\begin{aligned}
& \begin{array}{rlrl} 
& & 0.7309 \\
\text { CCY-cable: } 29970400902 & 331.54 \mathrm{~g} & =0.7307^{16} \\
\text { Shorting plug: } 28680100902 & 88.0903 \mathrm{~g} & =0.1942 \mathrm{lb}
\end{array} \\
& \text { Fastemers + Bracket: MM 130085-001 Rev }=1124.0842,=0.2736, \\
& \text { Aggregate weight (including fasteners and connector protective caps): } \\
& 0.2736 \text { it }
\end{aligned}
$$$$
1.1987^{\mathrm{lb}}[\mathrm{N} 6]
$$$$
\text { As-tested MMRTG weight [N5]+[N6]: 100:117_ lb [N7] }
$$

\begin{tabular}{l}
$\begin{array}{l}\text { The as-tested MMR'TG weight has been determined and recorded for use in the CG-location } \\
\text { calculations. }\end{array}$ \\
\hline SME/TL:
\end{tabular}

NOTE: $\quad$ The data in Lines 4(b) and 5, below, are not required to determine the as-tested weight and $C G$ location in this procedure. Those steps may be completed by the design agency later.

b. Aggregate center of gravity (CG) location:

$\mathrm{X}$-direction datum:

CG offset from datum: in.

Y-direction datum:

$\mathrm{CG}$ offset from datum: in.

Z-direction datum:

CG offset from datum in. 